\title{
Enzymatic Stetter Reaction: Computational Study of the Reaction Mechanism of MenD
}

\author{
Ferran Planas $^{a^{*}}$, Michael J. McLeish ${ }^{b}$ and Fahmi Himo ${ }^{a^{*}}$
}

a Department of Organic Chemistry, Arrhenius Laboratory, Stockholm University, SE-10691, Stockholm, Sweden.

${ }^{\mathrm{b}}$ Department of Chemistry and Chemical Biology, Indiana University-Purdue University Indianapolis, Indianapolis, IN 46202, USA.

E-mail corresponding authors: ferran.padros@su.se ; fahmi.himo@su.se

\section{Table of Contents}

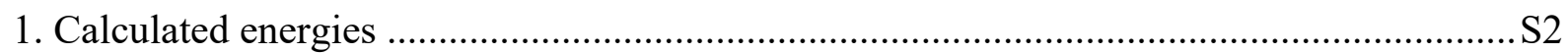

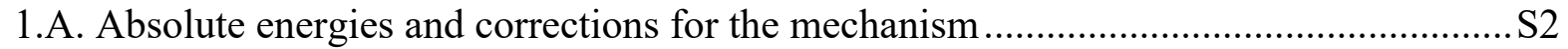

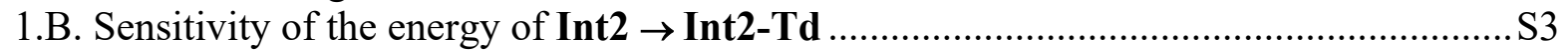

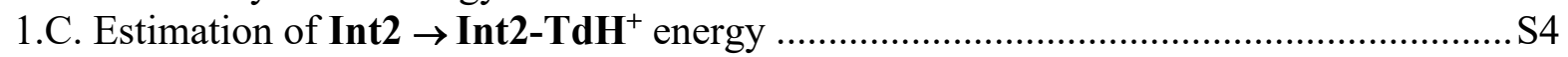

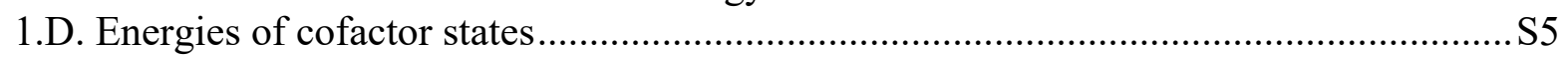

1.E. Energies of nucleophilic addition ...................................................................... S6

1.F. Energies of second half-reaction starting from an enolate ........................................ 7

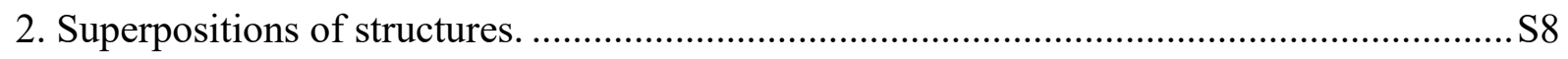

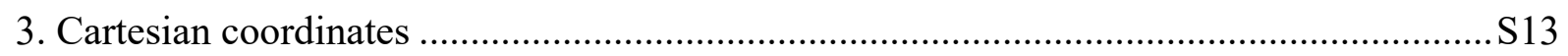




\section{Calculated energies}

In the tables below, the values are defined as follows:

- $\quad \mathbf{E}_{\mathbf{e l}}$ : Electronic energy calculated with the B3LYP-D3(BJ) functional using the 6-31G(d,p) basis set.

- $\quad \mathbf{E}_{\text {solv: }}$ Single-point solvation energy calculated using the SMD method at the same level of theory as the geometry optimization, with a dielectric constant $\varepsilon=4$.

- $\quad \mathbf{E}_{\mathbf{b b}}$ : Single-point large-basis set electronic energy, calculated with the 6-311+G(2d,2p) basis set.

- ZPE: Zero-point energy correction calculated at the same level of theory as the geometry optimization.

- $\quad$ The total energy is calculated using $\mathbf{E}_{\text {tot }}=\mathbf{E}_{\mathbf{b b}}+\left(\mathbf{E}_{\mathbf{s o l v}}-\mathbf{E}_{\mathbf{e l}}\right)+\mathbf{Z P E}$.

\section{A. Absolute energies and corrections for the mechanism}

Table S1. Calculated energies and energy corrections for the reaction mechanism. For the cofactor states and the first half-reaction, $\boldsymbol{E}_{r e l}$ is the energy relative to $\boldsymbol{Y I}$. For the second half-reaction, $\boldsymbol{E}_{\text {rel }}$ is calculated relative to Int3.

\begin{tabular}{|c|c|c|c|c|c|}
\hline & Eel $_{\text {el (a.u.) }}$ & E solv $_{\text {(a.u.) }}$ & $\mathbf{E}_{b b}$ (a.u.) & ZPE (a.u.) & $E_{\text {tot }}$ (a.u.) \\
\hline \multicolumn{6}{|l|}{ Cofactor states } \\
\hline YIH $^{+*}$ & -7524.31944 & -7524.47966 & -7526.43052 & 2.85615 & -7523.73459 \\
\hline TS-YIH $^{+*}$ & -7524.30447 & -7524.46397 & -7526.41609 & 2.85093 & -7523.72465 \\
\hline TC & -7524.33713 & -7524.48511 & -7526.44615 & 2.85500 & -7523.73913 \\
\hline $\mathrm{TCH}^{+*} *$ & -7524.31737 & -7524.47704 & -7526.42872 & 2.85500 & -7523.73338 \\
\hline TS-TCH $^{+*}$ & -7524.30117 & -7524.46075 & -7526.41362 & 2.85391 & -7523.71930 \\
\hline IP* & -7524.31219 & -7524.47524 & -7526.42780 & 2.85406 & -7523.73679 \\
\hline \multicolumn{6}{|c|}{ First-half reaction } \\
\hline YI & -7524.34061 & -7524.48934 & -7526.44711 & 2.85615 & -7523.73969 \\
\hline TS1 & -7524.32371 & -7524.47347 & -7526.42855 & 2.85551 & -7523.72280 \\
\hline Int1 & -7524.35894 & -7524.50651 & -7526.46052 & 2.85783 & -7523.75026 \\
\hline OC* & -7524.34329 & -7524.50449 & -7526.44936 & 2.85783 & -7523.75273 \\
\hline TS2 & -7524.33023 & -7524.47824 & -7526.43629 & 2.85783 & -7523.72646 \\
\hline Int2 & -7335.73697 & -7335.88801 & -7337.78332 & 2.84041 & -7335.09395 \\
\hline $\mathrm{CO}_{2}$ & -188.58258 & & -188.65198 & 0.01159 & -188.64039 \\
\hline Int2-Td & -7335.70593 & -7335.86419 & -7337.75801 & 2.84039 & -7335.07589 \\
\hline Int2-TdH ${ }^{+}$ & -7336.11797 & -7336.34405 & -7338.16127 & 2.85480 & -7335.53256 \\
\hline $\begin{array}{l}\text { Int2-TdH }^{+} \\
\left(\mathrm{N}^{\prime}-\mathrm{C} 2 \alpha=3.0 \AA\right)\end{array}$ & -7336.11541 & -7336.34172 & -7338.15885 & 2.85500 & -7335.53016 \\
\hline \multicolumn{6}{|c|}{ Second-half reaction } \\
\hline Int3 & -7946.15858 & -7946.31042 & -7948.36911 & 2.97796 & -7945.54298 \\
\hline TS4 & -7946.15346 & -7946.30552 & -7948.36186 & 2.98020 & -7945.53372 \\
\hline Int4 & -7946.15347 & -7946.30915 & -7948.36303 & 2.97975 & -7945.53896 \\
\hline TS5 & -7946.14285 & -7946.29757 & -7948.35037 & 2.97536 & -7945.52973 \\
\hline Int5 & -7946.16467 & -7946.31854 & -7948.37015 & 2.98046 & -7945.54356 \\
\hline TS6** & -7946.15286 & -7946.30475 & -7948.35810 & - & -7948.50999 \\
\hline $\mathbf{E P} * *$ & -7946.15956 & -7946.30910 & -7948.36839 & - & -7948.51793 \\
\hline
\end{tabular}

* Optimization of these geometries results in a proton transfer from N1' to Glu55. In order to obtain the energy of these species, the N1'-H distance was constrained at $1.10 \AA$.

** The energy of TS6 is estimated from as the maximum in energy obtained from stepping the C2-C2 $\alpha$ bond, and was found to be a distance of $1.90 \AA$. The energy of $\mathbf{E P}$ is estimated from a geometry with the carbonyl group of the product constrained to be planar. In both cases, the energies are calculated relative to Int5 and do not include the ZPE correction. 


\section{B. Sensitivity of the energy of Int $2 \rightarrow$ Int2-Td}

In order to evaluate the sensitivity of the Int2 $\rightarrow$ Int2-Td step to the protonation state of the ionizable groups in the active site, the energy of this step was calculated with different protonation states of $\mathrm{C} 2 \alpha \mathrm{O}, \mathrm{N} 1$ ' and Glu55. The obtained energies are reported in Table $\mathbf{S 2}$. As seen, the calculations show that the effects are rather small, and, importantly, the reaction is always endothermic, by at least $6 \mathrm{kcal} / \mathrm{mol}$.

Table S2. Calculated energies and energy corrections for the Int2 $\rightarrow$ Int2-Td step using different protonation states of Glu55, N1' and C2 $\alpha O$. $\boldsymbol{E}_{\text {rel }}$ the energy relative to Int2 in each model.

\begin{tabular}{|c|c|c|c|c|c|c|}
\hline & Eel (a.u.) & Esolv (a.u.) & Ebb (a.u.) & ZPE (a.u.) & E $_{\text {tot }}$ (a.u.) & 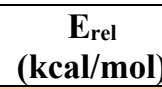 \\
\hline \multicolumn{7}{|c|}{ Model A: C2 $\alpha \mathrm{O}$, N1', Glu55-OH. } \\
\hline Int2 & -7335.73697 & -7335.88801 & -7337.78332 & 2.84041 & -7335.09395 & 0.0 \\
\hline Int2-Td & -7335.70593 & -7335.86419 & -7337.75801 & 2.84039 & -7335.07589 & 11.3 \\
\hline \multicolumn{7}{|c|}{ Model B: C2 $\alpha \mathrm{OH}, \mathrm{N1}^{\prime}$, Glu55-OH. } \\
\hline Int2 & -7336.12756 & -7336.35206 & -7338.16833 & 2.85430 & -7335.53852 & 0.0 \\
\hline Int2-Td & -7336.10474 & -7336.33008 & -7338.15103 & 2.85266 & -7335.52372 & 9.3 \\
\hline \multicolumn{7}{|c|}{ Model C: C2 $\alpha \mathrm{OH}, \mathrm{N1}$ 'H, Glu55-OH. } \\
\hline Int2 & -7336.47409 & -7336.79434 & -7338.51121 & 2.86620 & -7335.96525 & 0.0 \\
\hline Int2-Td & -7336.45249 & -7336.77758 & -7338.49416 & 2.86382 & -7335.95544 & 6.2 \\
\hline
\end{tabular}




\section{C. Estimation of Int2 $\rightarrow$ Int2-TdH ${ }^{+}$energy}

In the case of the protonation of $\mathrm{C} 2 \alpha$ by a bulk proton $\left(\mathbf{I n t} 2 \rightarrow \operatorname{Int} 2-\mathbf{T d H} \mathbf{H}^{+}\right.$), the energy was estimated using the following approximation:

- $\Delta \mathrm{E}_{\text {solv }, Z P E}=\left[\mathrm{E}_{\text {solv }, Z P E}\left(\right.\right.$ Int2-TdH $\left.{ }^{+}\right)-E_{\text {solv,ZPE }}($ Int2) $] \times 627.5+279.8$

- $279.8 \mathrm{kcal} / \mathrm{mol}$ is the Gibbs free energy of a proton at $\mathrm{pH}=7$. The value is obtained from the translational entropy of a proton in the gas phase $(-6.3 \mathrm{kcal} / \mathrm{mol})$ plus the solvation energy of a proton in water $(-264 \mathrm{kcal} / \mathrm{mol})$ and the correction for $\mathrm{pH}(7 \mathrm{x}-1.36$ $\mathrm{kcal} / \mathrm{mol})$.

The used estimation depends on the choice of the dielectric constant. In Table S3 the Int2 $\rightarrow$ Int2-TdH ${ }^{+}$energies calculated with different dielectric constants are listed. As seen, the calculations show that the step is always exothermic, regardless of the chosen dielectric constant.

Table S3. Calculated reaction energy for the Int $\rightarrow \boldsymbol{I n t} \mathbf{2}-\mathbf{T d H}^{+}$using different dielectric constants.

\begin{tabular}{|c|c|c|c|}
\hline \multirow{2}{*}{ Dielectric constant } & \multicolumn{2}{|c|}{$\mathbf{E}_{\text {solv,ZPE (a.u.) }}$} & \multirow{\Delta}{*}{$\begin{array}{c}\Delta \mathbf{E}_{\text {solv,ZPE }} \\
\text { (kcal/mol) }\end{array}$} \\
\cline { 2 - 3 } & $\mathbf{I n t 2}$ & Int2-TdH & -1.4 \\
\hline $\boldsymbol{\varepsilon}=\mathbf{4}$ & -7335.08444 & -7335.53255 & -8.3 \\
\hline $\boldsymbol{\varepsilon}=\mathbf{8}$ & -7335.12875 & -7335.58788 & -11.4 \\
\hline $\boldsymbol{\varepsilon}=\mathbf{1 6}$ & -7335.15461 & -7335.61874 & -12.9 \\
\hline $\boldsymbol{\varepsilon}=\mathbf{3 2}$ & -7335.16895 & -7335.63537 & -13.7 \\
\hline $\boldsymbol{\varepsilon}=\mathbf{8 0}$ & -7335.17817 & -7335.64587 & \\
\hline
\end{tabular}




\section{D. Energies of cofactor states}

We have evaluated the effect of the protonation state of $\mathrm{N} 1$ ' on the cofactor energies. As shown in Tables $\mathbf{S} 4$ the energy changes are not significant, indicating that the protonation state of N1' does not have a major impact on the formation of the mentioned intermediates. Note that in this case the ZPE correction have not been added.

Table S4. Calculated reaction energy for the IP $\rightarrow$ YI transformation using different protonation states of N1' and Glu55.

\begin{tabular}{|c|c|c|c|c|c|}
\hline & $E_{\text {el }}($ a.u. $)$ & Esolv (a.u.) & $\mathbf{E}_{b b}$ (a.u.) & $\mathbf{E}_{\text {tot }}$ (a.u.) & $\begin{array}{c}\text { Erel }_{\text {rel }} \\
(\mathrm{kcal} / \mathrm{mol})\end{array}$ \\
\hline \multicolumn{6}{|c|}{ Model A: N1', Glu55-OH. } \\
\hline IP & -7524.32578 & -7524.47698 & -7526.43809 & -7526.58929 & 0.0 \\
\hline TS & -7524.32042 & -7524.46790 & -7526.42894 & -7526.57642 & 8.1 \\
\hline YI & -7524.34061 & -7524.48934 & -7526.44711 & -7526.59583 & -4.1 \\
\hline \multicolumn{6}{|c|}{ Model B: N1'H, Glu55-O. } \\
\hline IP & -7524.31219 & -7524.47524 & -7526.42780 & -7526.59085 & 0.0 \\
\hline TS & -7524.30447 & -7524.46397 & -7526.41609 & -7526.57559 & 9.6 \\
\hline YI & -7524.31944 & -7524.48166 & -7526.43052 & -7526.59074 & -1.2 \\
\hline \multicolumn{6}{|c|}{ Model C: N1'H, Glu55-OH. } \\
\hline IP & -7524.73132 & -7524.94444 & -7526.83177 & -7527.04488 & 0.0 \\
\hline TS & -7524.71933 & -7524.93157 & -7526.81729 & -7527.02953 & 9.6 \\
\hline YI & -7524.73375 & -7524.95054 & -7526.83208 & -7527.04687 & -2.5 \\
\hline
\end{tabular}




\section{E. Energies of nucleophilic addition}

We also evaluated how the energies of the formation of the first tetrahedral intermediate change depending on the protonation state of N1' and Glu55. Tables S5 show that also this step is rather insensitive to the protonation state of $\mathrm{N} 1$ '.

Table S5. Calculated reaction energy for the $Y I \rightarrow I n t 1$ transformation using different protonation states of N1' and Glu55.

\begin{tabular}{|c|c|c|c|c|c|}
\hline & Eel (a.u.) & Esolv (a.u.) & Ebb (a.u.) & Etot (a.u.) & $\begin{array}{c}\text { Erel } \\
\text { (kcal/mol) }\end{array}$ \\
\hline Model A: N1', Glu55-OH. & \multicolumn{5}{|l|}{} \\
\hline YI & -7524.34061 & -7524.48934 & -7526.44711 & -7526.59583 & $\mathbf{0 . 0}$ \\
\hline TS1 & -7524.32371 & -7524.47347 & -7526.42855 & -7526.57831 & $\mathbf{1 1 . 0}$ \\
\hline Int1 & -7524.35894 & -7524.50651 & -7526.46052 & -7526.60810 & $-\mathbf{7 . 7}$ \\
\hline \multicolumn{7}{|l|}{} \\
\hline Model B: N1'H, Glu55-O. \\
\hline YI & -7524.31944 & -7524.48166 & -7526.43052 & -7526.59074 & $\mathbf{0 . 0}$ \\
\hline TS1 & -7524.30687 & -7524.46605 & -7526.41292 & -7526.57210 & $\mathbf{1 2 . 9}$ \\
\hline Int1 & -7524.34427 & -7524.50135 & -7526.44852 & -7526.60560 & $\mathbf{- 8 . 0}$ \\
\hline \multicolumn{7}{|l|}{} \\
\hline Model C: N1'H, Glu55-OH. & & & \\
\hline YI & -7524.73375 & -7524.95054 & -7526.83208 & -7527.04687 & $\mathbf{0 . 0}$ \\
\hline TS1 & -7524.72812 & -7524.93898 & -7526.82242 & -7527.03328 & $\mathbf{9 . 8}$ \\
\hline Int1 & -7524.75808 & -7524.96741 & -7526.85518 & -7527.06652 & $\mathbf{- 9 . 8}$ \\
\hline
\end{tabular}




\section{F. Energies of second half-reaction starting from an enolate}

The second part of the reaction mechanism was also calculated starting from a model with the enolate form of the Breslow intermediate instead of the enol in the donor:acceptor complex. Table S6 shows the electronic energies for the linear transit for the cleavage of the C2-C2 $\alpha$ bond. The energies for the enolate case are considerably higher than for the enol case.

Table S6. Energies of the linear transit for the cleavage of the C2-C2 $\alpha$ bond to form the SEPHCHC product.

\begin{tabular}{|l|c|c|c|c|}
\hline \multirow{2}{*}{} & \multicolumn{3}{|c|}{ Protonation of the Breslow intermediate } \\
\cline { 2 - 5 } & \multicolumn{2}{|c|}{ Enolate } & \multicolumn{2}{c|}{ Enol } \\
\cline { 2 - 5 } & $\mathbf{E}_{\text {el }}$ (a.u.) & $\begin{array}{c}\mathbf{E}_{\text {rel }} \\
\text { (kcal/mol) }\end{array}$ & Eel $_{\text {(a.u.) }}$ & $\begin{array}{c}\mathbf{E}_{\text {rel }} \\
\text { (kcal/mol) }\end{array}$ \\
\hline Int5 & -7945.65641 & 0.0 & -7946.16033 & 0.0 \\
\hline C-C: $1.9 \AA$ & -7945.63961 & +10.5 & -7946.15286 & +4.7 \\
\hline C-C: $2.2 \AA$ & -7945.63638 & +12.5 & -7946.16037 & 0.0 \\
\hline C-C: $2.4 \AA$ & -7945.63522 & +13.3 & -7946.16097 & -0.4 \\
\hline C-C: $3.0 \AA$ & -7945.63438 & +13.8 & -7946.15956 & +0.5 \\
\hline
\end{tabular}




\section{Superpositions of structures.}

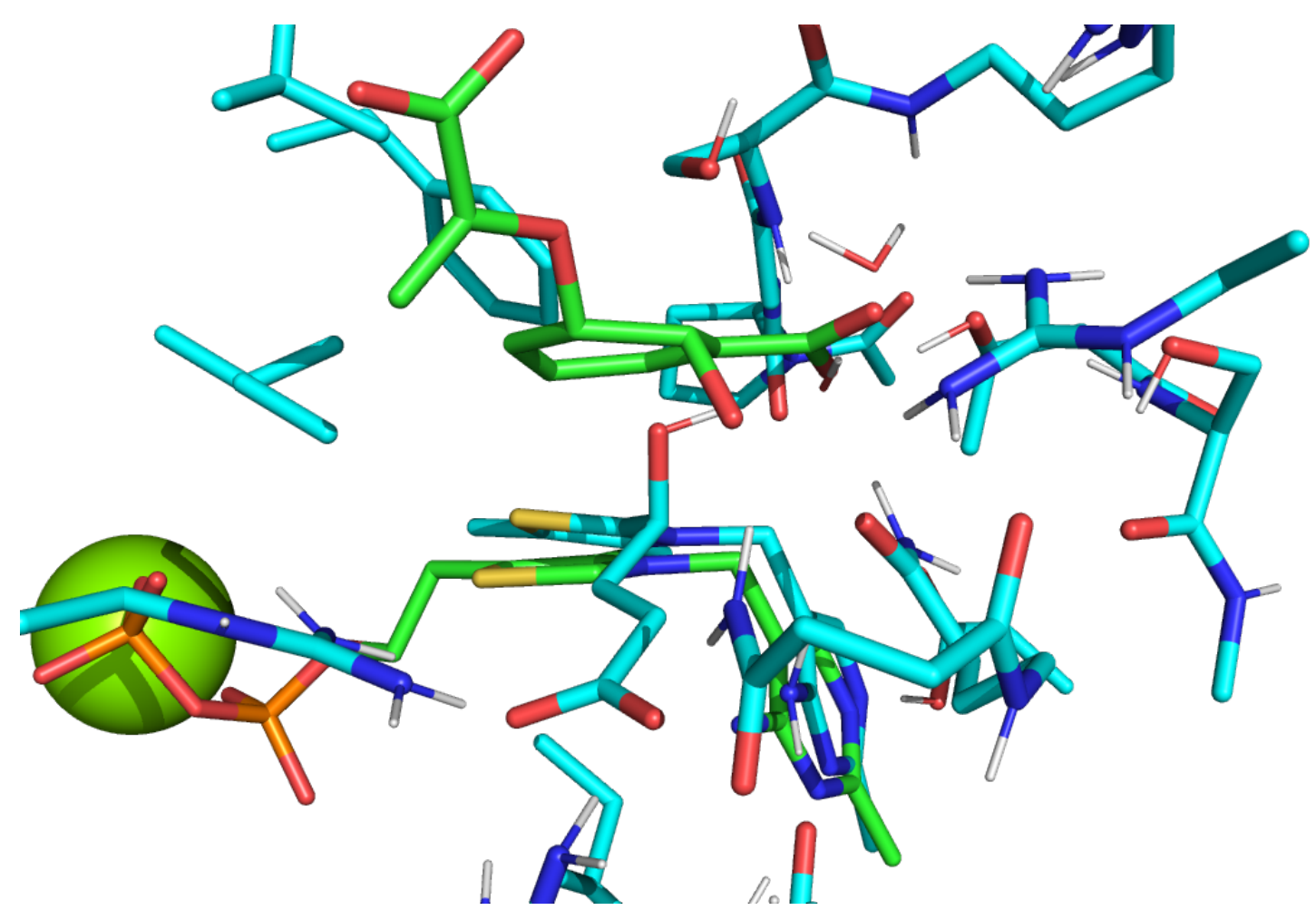

Figure S1. Superposition of the calculated Int2-TdH $\boldsymbol{H}^{+}$(cyan) with the enzyme-isochorismate complex (green, PDB 5ESO). For clarity, all non-polar hydrogens are omitted. 


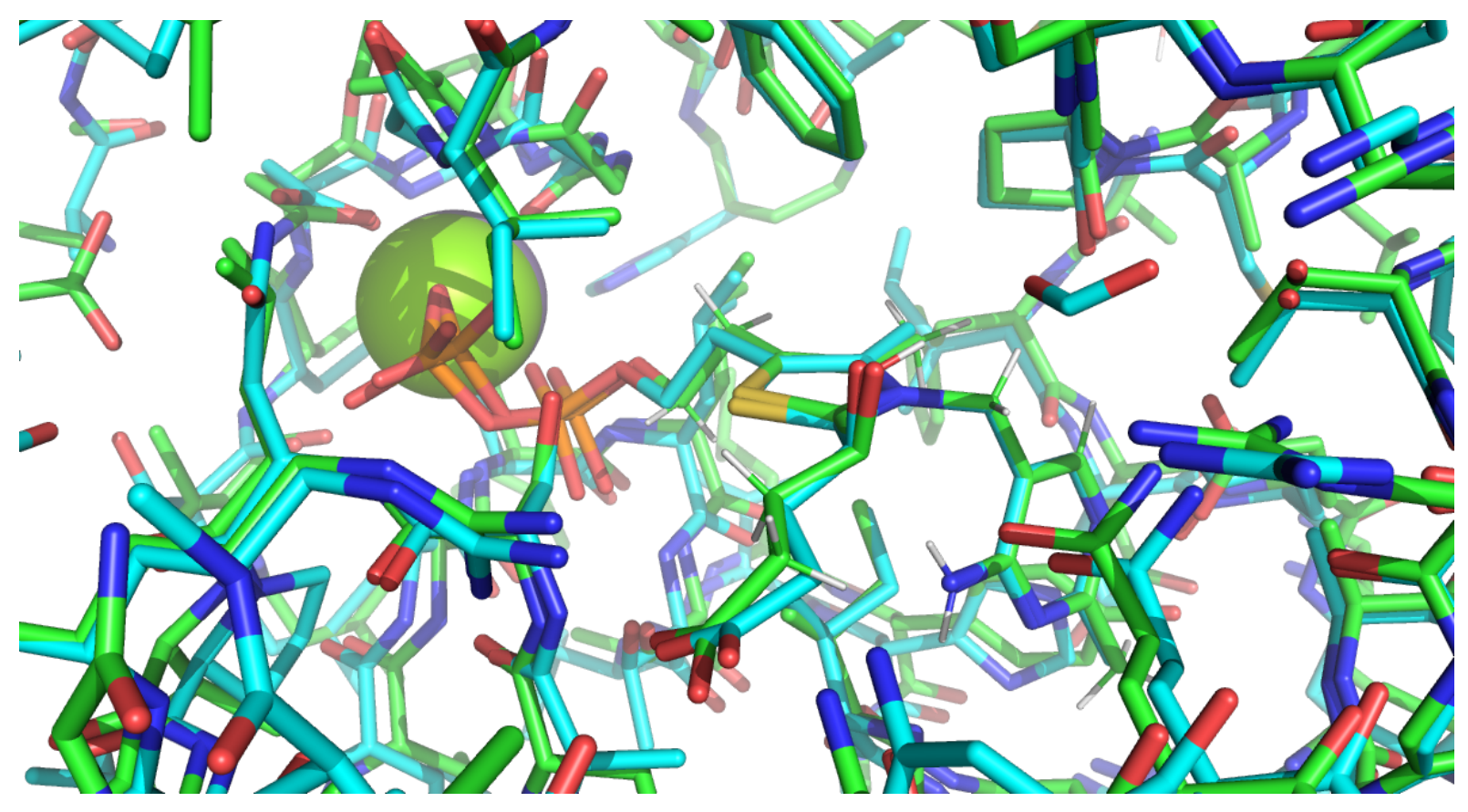

Figure S2. Superposition of the crystal structures of the tetrahedral post-decarboxylation intermediate in E.coli (green, PDB 5EJ5) and M.tuberculosis (cyan, PDB 5ESS). 


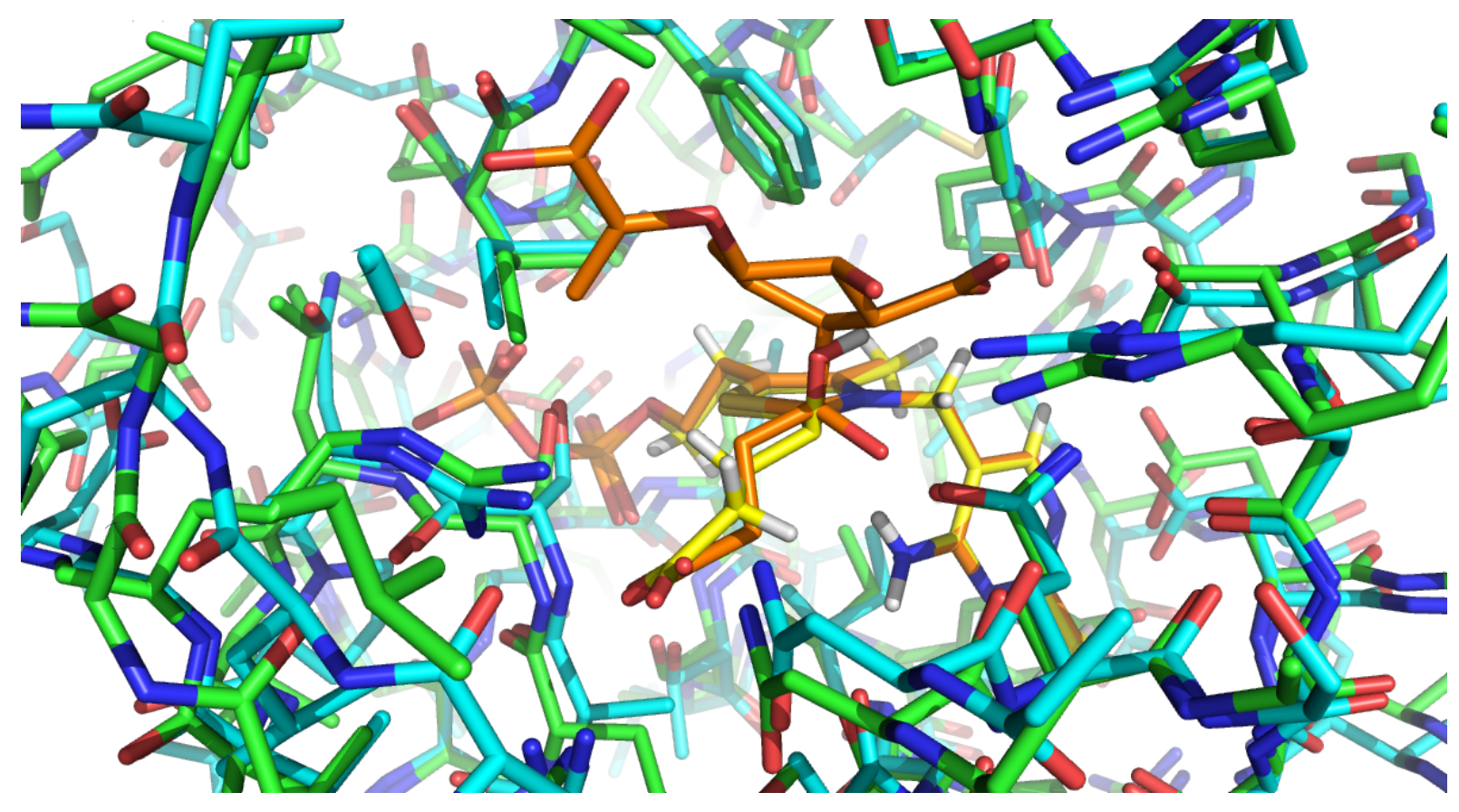

Figure S3. Superposition of the tetrahedral post-decarboxylation intermediate in E.coli (green/yellow, PDB 5EJ5) and second covalent intermediate from M.tuberculosis. (cyan/orange PDB 5ESU). 


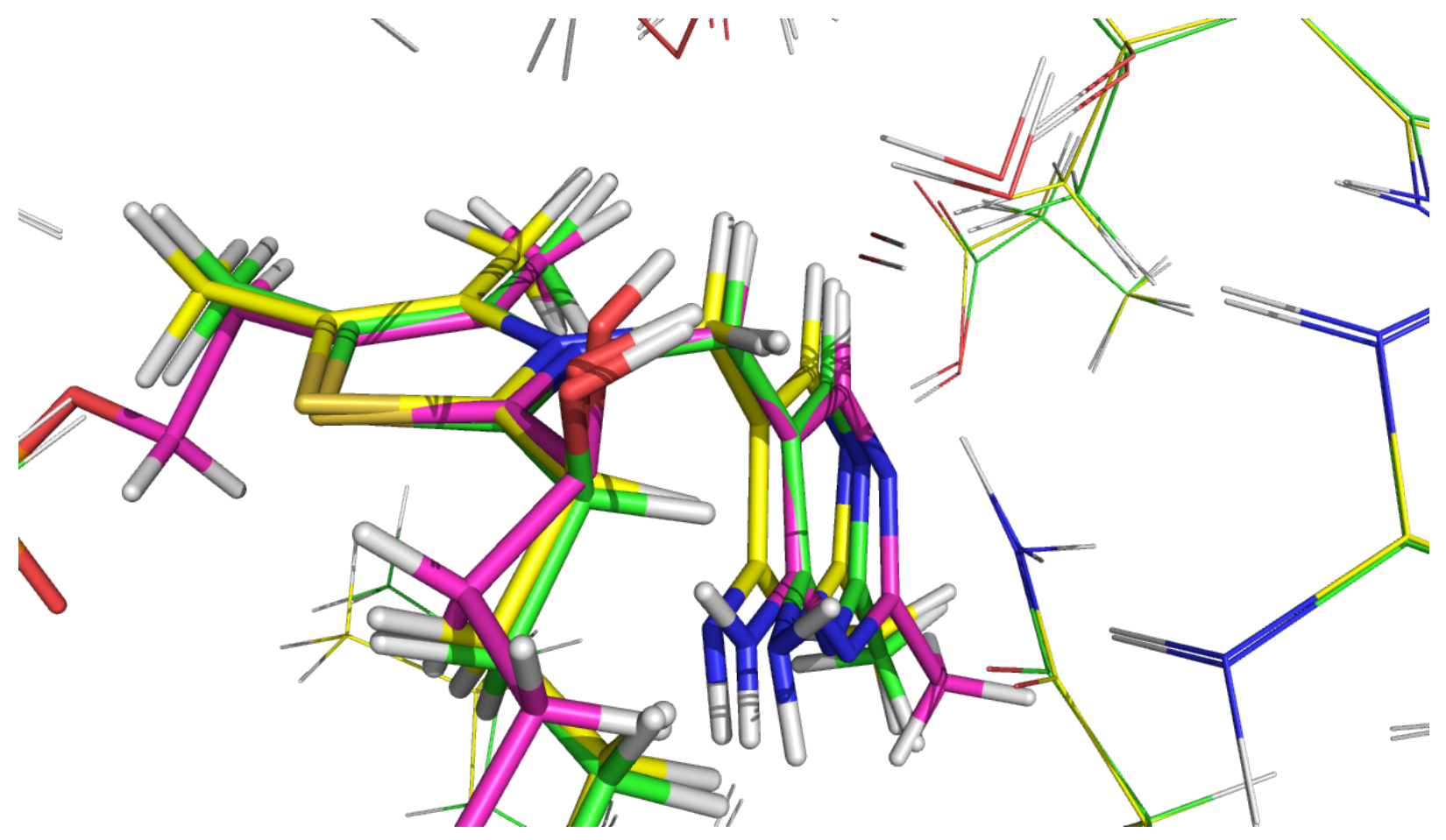

Figure S4. Superposition of the post-decarboxylation intermediates: the calculated Int2-Td (Yellow), Int2-TdH ${ }^{+}$with the $\mathrm{N}^{\prime}$ '-C2 $\alpha$ distance restrained at $3.0 \AA$, and the crystal structure (pink). 


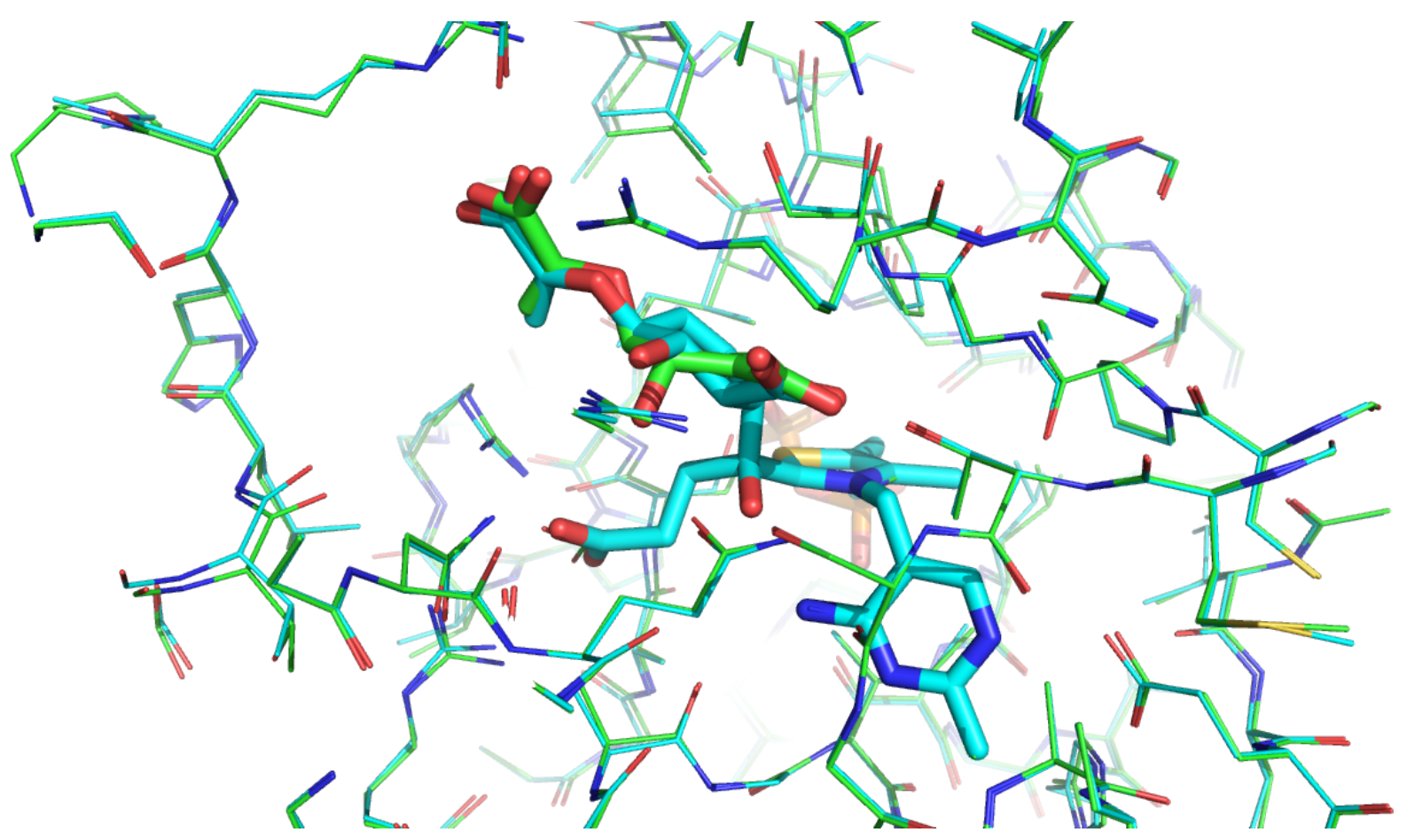

Figure S5. Superposition of the second covalent intermediate (cyan, PDB 5ESU) with the enzyme-isochorismate complex (green, PDB 5ESO). 


\section{Cartesian coordinates}

\section{TS for $\mathbf{I P} \rightarrow \mathbf{T C H}^{+}$}

$\begin{array}{llll}\mathrm{N} & -2.2243 & -5.2990 & 0.4622\end{array}$

C $-3.3045-5.2056-1.7452$

$\begin{array}{llll}\text { C } & -4.2392 & -5.8892 & -2.7512\end{array}$

$\begin{array}{llll}\text { C } & -5.7041 & -6.0080 & -2.3222\end{array}$

N $-6.3193-4.6901-2.1963$

$\begin{array}{llll}\text { C } & -7.6530 & -4.5039 & -2.0669\end{array}$

$\begin{array}{llll}\mathrm{N} & -8.4976 & -5.5152 & -2.2849\end{array}$

N $-8.1529-3.2736-1.8492$

C $-11.1101 \quad-5.1930 \quad 2.1430$

$\begin{array}{llll}\text { C } & -9.5852 & -5.0677 & 2.1725\end{array}$

$\begin{array}{llll}\text { C } & -8.9471 & -4.4752 & 0.9105\end{array}$

$\begin{array}{llll}\text { O } & -7.7580 & -4.7253 & 0.6590\end{array}$

$\begin{array}{llll}\text { O } & -9.7144 & -3.7146 & 0.1797\end{array}$

O - $11.1212-5.1962-1.4697$

$\begin{array}{llll}\text { O } & -2.8300 & -1.8459 & -0.0286\end{array}$

$\begin{array}{llll}\text { C } & 8.1824 & -6.7600 & -0.5544\end{array}$

$\begin{array}{llll}C & 6.7464 & -6.6079 & -0.0563\end{array}$

$\begin{array}{llll}\text { O } & 5.7787 & -6.9458 & -0.7328\end{array}$

$\begin{array}{llll}\mathrm{N} & 6.5645 & -6.0595 & 1.1916\end{array}$

C $5.2377-6.0735 \quad 1.8050$

C 4.4064 -4.8197 1.4967

$\begin{array}{llll}\text { O } & 4.9085 & -3.6893 & 1.5074\end{array}$

C $5.5606-6.0972 \quad 3.3160$

C $6.8563 \quad-5.2769 \quad 3.4038$

$\begin{array}{llll}\text { C } & 7.6234 & -5.6847 & 2.1372\end{array}$

N $3.0853 \quad-5.0346 \quad 1.3141$

$\begin{array}{llll}\text { C } & 2.0921 & -3.9754 & 1.3049\end{array}$

$\begin{array}{llll}\text { C } & 0.7795 & -4.5441 & 1.8473\end{array}$

$\begin{array}{lllll}\text { O } & 0.7230 & -5.6871 & 2.2981\end{array}$

$\begin{array}{llll}\mathrm{N} & -0.3020 & -3.7193 & 1.7761\end{array}$

$\begin{array}{llll}\text { C } & -1.5261 & -4.0713 & 2.4898\end{array}$

$\begin{array}{llll}\text { C } & -2.5894 & -4.8052 & 1.6607\end{array}$

$\begin{array}{llll}\text { O } & -3.7280 & -4.9325 & 2.1284\end{array}$

$\begin{array}{llll}\text { C } & -2.1817 & -2.8574 & 3.2111\end{array}$

$\begin{array}{llll}\text { O } & -3.3465 & -2.3417 & 2.5611\end{array}$

$\begin{array}{llll}\text { C } & 9.1119 & -3.2512 & -6.0572\end{array}$

$\begin{array}{llll}\text { C } & 9.3552 & -3.5121 & -4.5682\end{array}$

$\begin{array}{llll}\text { C } & 8.5386 & -2.5769 & -3.6717\end{array}$

$\begin{array}{lllll}\text { O } & 8.4822 & -1.3549 & -4.0664\end{array}$

$\begin{array}{llll}\text { O } & 7.9913 & -3.0381 & -2.6445\end{array}$

$\begin{array}{llll}\text { C } & 1.4545 & -5.3260 & -3.3932\end{array}$

$\begin{array}{llll}C & 0.8287 & -4.7347 & -4.6557\end{array}$

$\begin{array}{llll}\text { O } & 1.0846 & -5.1500 & -5.7810\end{array}$

$\begin{array}{llll}\text { C } & 2.0836 & -4.3021 & -2.4441\end{array}$

$\begin{array}{llll}\text { C } & 3.0838 & -3.3979 & -3.1607\end{array}$

$\begin{array}{llll}\text { O } & 1.0206 & -3.5677 & -1.8146\end{array}$

N $-0.0737-3.7148$ - 4.4516

$\begin{array}{llll}\text { C } & -0.9139 & -3.2594 & -5.5416\end{array}$

$\begin{array}{llll}\text { C } & -0.6960 & -1.7817 & -5.9159\end{array}$

$\begin{array}{llll}\text { O } & -1.2637 & -0.8743 & -5.2808\end{array}$

$\begin{array}{llll}\text { C } & -2.3989 & -3.4539 & -5.2076\end{array}$

$\begin{array}{lllll}\text { O } & -2.7637 & -2.7181 & -4.0449\end{array}$

$\begin{array}{lllll}\mathrm{N} & 0.0577 & -1.5379 & -7.0047\end{array}$

C $0.2501 \quad-0.1979-7.5586$
C $-7.7006-2.0506-5.1975$

C $-6.2715-2.0303-4.6430$

$\begin{array}{llll}\mathrm{N} & -6.0369 & -0.8695 & -3.7727\end{array}$

C $-5.3545-0.8693-2.6210$

N $-4.6903-1.9674 \quad-2.2092$

$\mathrm{N}-5.4011 \quad 0.2173 \quad-1.8303$

C $-6.2436 \quad 4.5416-3.6652$

C $-5.5447 \quad 3.2664-4.1239$

$\begin{array}{lllll}\text { O } & -5.9884 & 2.1579 & -3.7863\end{array}$

C $-5.9737 \quad 4.7602 \quad-2.1587$

C $-4.5800 \quad 5.2858-1.8166$

$\mathrm{N}-3.7051 \quad 4.4155 \quad-1.3001$

$\begin{array}{lllll}\mathrm{O} & -4.3179 & 6.4985 & -1.9770\end{array}$

$\begin{array}{llll}\mathrm{N} & -4.4046 & 3.4182 & -4.8319\end{array}$

C $-\begin{array}{llll}-3.3533 & 2.3931 & -4.9024\end{array}$

C $-2.1006 \quad 2.9142-4.1624$

$\begin{array}{llll}\text { C } & -1.2820 & 1.8373 & -3.4547\end{array}$

$\begin{array}{llll}C & -2.0919 & 1.1203 & -2.3915\end{array}$

$\mathrm{N}-1.8126 \quad-0.1813 \quad-2.2219$

$\begin{array}{lllll}\text { O } & -2.9919 & 1.6801 & -1.7268\end{array}$

$\begin{array}{llll}\text { O } & 1.0229 & -0.9218 & -1.9213\end{array}$

$\begin{array}{llll}\text { C } & -3.0744 & 8.1364 & 6.4774\end{array}$

$\begin{array}{llll}\text { C } & -2.4137 & 6.7629 & 6.6283\end{array}$

$\mathrm{N}-2.8430 \quad 5.7915 \quad 5.6181$

C $-2.3904 \quad 5.7548 \quad 4.3507$

$\mathrm{N}-1.4933 \quad 6.6398 \quad 3.9177$

$\mathrm{N}-2.8556 \quad 4.8234 \quad 3.5110$

C $-1.7061 \quad 11.8225-1.8828$

C $-1.0943 \quad 10.5144-1.4061$

$\begin{array}{llll}\mathrm{N} & -2.1213 & 9.6792 & -0.7828\end{array}$

$\begin{array}{llll}\text { C } & -1.8455 & 8.5818 & -0.0556\end{array}$

$\begin{array}{llll}\mathrm{N} & -2.8192 & 7.7035 & 0.2183\end{array}$

$\begin{array}{llll}\mathrm{N} & -0.6361 & 8.4148 & 0.4840\end{array}$

$\begin{array}{llll}\text { C } & 0.5118 & 6.4549 & -3.4369\end{array}$

C $1.4085 \quad 5.2223 \quad-3.3861$

O $1.1518 \quad 4.2415 \quad-2.6837$

C $-0.7289 \quad 6.2778-2.5659$

$\begin{array}{lllll}\mathrm{O} & -1.6229 & 7.3654 & -2.7803\end{array}$

N $2.4907 \quad 5.2966-4.2103$

C $3.6506 \quad 4.4545 \quad-3.9978$

C $4.7388 \quad 5.3054-3.3243$

$\begin{array}{lllll}\text { O } & 4.6963 & 6.5339 & -3.4066\end{array}$

N $5.7034 \quad 4.6080-2.6916$

C $6.7681 \quad 5.2718-1.9427$

C $\quad 6.9410 \quad 4.6638 \quad-0.5377$

C $7.5708 \quad 3.2603 \quad-0.6134$

$\begin{array}{llll}\text { C } & 5.6153 & 4.6686 & 0.2373\end{array}$

$\begin{array}{llll}\text { C } & 7.8492 & 2.6088 & 0.7425\end{array}$

C $\quad 1.6191 \quad 1.9508 \quad 8.8686$

$\begin{array}{llll}\text { C } & 1.5921 & 2.2742 & 7.3657\end{array}$

C $\quad 0.1795 \quad 2.0489 \quad 6.7932$

$\begin{array}{llll}\text { C } & 2.6581 & 1.4626 & 6.6169\end{array}$

$\begin{array}{llll}\text { C } & 0.0028 & 2.4473 & 5.3239\end{array}$

$\begin{array}{llll}\text { C } & 1.6252 & -1.8381 & 9.2531\end{array}$

$\begin{array}{llll}\text { C } & 1.7880 & -2.9048 & 8.1541\end{array}$

C $1.8009-2.3545 \quad 6.7412$

$\begin{array}{llll}\text { C } & 0.6679 & -1.7179 & 6.2134\end{array}$

C $2.9277-2.4808 \quad 5.9187$

C $0.6604-1.2168 \quad 4.9125$

C $2.9249-1.9949 \quad 4.6074$
C $1.7901-1.3600 \quad 4.1026$

$\begin{array}{llll}\text { C } & -3.3100 & -1.7963 & 10.3340\end{array}$

C $-3.4575-1.7475 \quad 8.8096$

C $-2.7060 \quad-0.5966 \quad 8.1155$

$\begin{array}{llll}\text { C } & -2.8383 & -0.7106 & 6.5913\end{array}$

$\begin{array}{llll}\text { C } & -3.1786 & 0.7823 & 8.5922\end{array}$

$\begin{array}{llll}\text { C } & -0.7742 & 2.5963 & 1.1820\end{array}$

$\begin{array}{llll}\text { C } & -0.6752 & 5.0976 & 1.1268\end{array}$

$\begin{array}{lllll}\text { O } & 0.2978 & 1.3998 & -0.5679\end{array}$

$\begin{array}{llll}\mathrm{O} & -1.9347 & 5.1867 & 0.9087\end{array}$

$\begin{array}{llll}\text { O } & -0.0117 & 6.0165 & 1.6936\end{array}$

C $\quad 6.9471 \quad 1.7304 \quad-4.1668$

$\begin{array}{llll}\text { C } & 6.1813 & -0.7250 & 1.8599\end{array}$

C $4.1456-1.3346-0.2411$

$\begin{array}{llll}\text { C } & 6.0247 & -1.0039 & -1.8699\end{array}$

C $4.8666-0.5630-1.3013$

$\begin{array}{llll}\text { C } & 4.3543 & 0.7253 & -1.7094\end{array}$

N $3.1868 \quad 1.1126-1.2171$

$\begin{array}{llll}\mathrm{N} & 5.0747 & 1.4949 & -2.5988\end{array}$

C $6.1587 \quad 0.9643-3.1450$

N $6.6400 \quad-0.2461 \quad-2.8119$

$\begin{array}{llll}\text { S } & 2.9207 & 1.6920 & 1.9215\end{array}$

$\begin{array}{llll}\text { C } & 2.8602 & 0.4370 & 0.7222\end{array}$

N $3.8963 \quad-0.4140 \quad 0.8894$

$\begin{array}{llll}\text { C } & 4.9056 & 0.0439 & 1.7623\end{array}$

$\begin{array}{llll}\text { C } & 4.5404 & 1.1652 & 2.4294\end{array}$

$\begin{array}{llll}\text { C } & 5.2641 & 1.9295 & 3.4897\end{array}$

$\begin{array}{llll}\text { C } & -0.2868 & 1.3492 & 0.5006\end{array}$

$\begin{array}{llll}\text { C } & 0.0142 & 3.8241 & 0.7182\end{array}$

H $2.9138 \quad 2.0631-1.4652$

$\mathrm{H}-1.8318 \quad 2.7018 \quad 0.9053$

$\begin{array}{llll}\mathrm{H} & -0.7476 & 2.4510 & 2.2644\end{array}$

H $7.6343 \quad 2.4206 \quad-3.6659$

H $\quad 6.2795 \quad 2.3190 \quad-4.7976$

H $7.5420 \quad 1.0449 \quad-4.7736$

H $5.9811-1.7889 \quad 2.0113$

$\begin{array}{llll}\text { H } & 6.7887 & -0.3530 & 2.6849\end{array}$

H $\quad 6.7635 \quad-0.6249 \quad 0.9372$

H $4.7289 \quad 1.8909 \quad 4.4443$

$\begin{array}{llll}\mathrm{H} & 0.1060 & 3.8110 & -0.3751\end{array}$

H $6.5296-1.9556-1.6896$

H $3.1598 \quad-1.6636-0.5787$

H $\quad 6.2601 \quad 1.5117 \quad 3.6465$

$\begin{array}{llll}\mathrm{H} & 1.0271 & 3.8213 & 1.1244\end{array}$

H $4.6924-2.2072 \quad 0.1111$

H $-0.2160 \quad-0.7170 \quad 4.5160$

H $1.7761 \quad-0.9822 \quad 3.0862$

H $-0.2197 \quad-1.6189 \quad 6.8297$

H $3.8143 \quad-2.9760 \quad 6.3061$

H $3.8003 \quad-2.1263 \quad 3.9772$

H $0.9699 \quad-3.6309 \quad 8.2444$

H $2.7131 \quad-3.4643 \quad 8.3288$

H $\quad 0.6848 \quad-1.2908 \quad 9.1336$

H $-3.1018 \quad-2.6952 \quad 8.3854$

H $-4.5223-1.68018 .5457$

$\begin{array}{llll}\mathrm{H} & -4.2509 & 0.9098 & 8.3980\end{array}$

$\begin{array}{llll}\mathrm{H} & -3.0136 & 0.9247 & 9.6638\end{array}$

$\begin{array}{llll}\mathrm{H} & -2.6425 & 1.5794 & 8.0658\end{array}$

H $-1.6402-0.69018 .3757$

H $-3.8850 \quad-0.6045 \quad 6.2830$ 
$\begin{array}{llll}\mathrm{H} & -2.2585 & 0.0638 & 6.0773\end{array}$

$\begin{array}{llll}\mathrm{H} & -2.4891 & -1.6820 & 6.2277\end{array}$

H $-3.3256 \quad 4.0071 \quad 3.8690$

H $-2.6146 \quad 4.8684 \quad 2.5098$

H $-1.2008 \quad 7.3886 \quad 4.5222$

$\begin{array}{llll}\text { H } & -0.9439 & 6.4631 & 3.0527\end{array}$

$\begin{array}{llll}\mathrm{H} & -3.6701 & 5.2487 & 5.8184\end{array}$

H $-1.3216 \quad 6.8336 \quad 6.5885$

H $-2.6515 \quad 6.3277 \quad 7.6012$

H $-2.8616 \quad 8.5883 \quad 5.5042$

$\begin{array}{llll}\mathrm{H} & -4.1603 & 8.0567 & 6.5766\end{array}$

$\begin{array}{llll}\mathrm{H} & -2.5198 & 6.8093 & 0.6390\end{array}$

H $-3.5486 \quad 7.5780-0.4820$

H $-3.0127 \quad 9.6624-1.2595$

$\begin{array}{llll}\mathrm{H} & 0.1044 & 9.0594 & 0.2682\end{array}$

$\begin{array}{llll}\mathrm{H} & -0.3944 & 7.5351 & 0.9690\end{array}$

H $-0.6253 \quad 9.9623-2.2296$

H $-0.3279 \quad 10.7431-0.6588$

H $-0.9392 \quad 12.4486-2.3449$

H $-2.1494 \quad 12.3738-1.0495$

H $\quad 8.8892 \quad-7.0273 \quad 0.2340$

H $8.2834 \quad-6.5384 \quad 2.3391$

H $8.2365 \quad-4.8701 \quad 1.7404$

H $7.4280 \quad-5.4758 \quad 4.3136$

H $6.6177 \quad-4.2118 \quad 3.3662$

H $5.7344 \quad-7.1327 \quad 3.6259$

H $4.7485 \quad-5.6929 \quad 3.9260$

H $4.6982-6.9606 \quad 1.4646$

H $2.7002 \quad-5.9574 \quad 1.4857$

$\begin{array}{llll}\text { H } & 1.9107 & -3.5894 & 0.2959\end{array}$

H $2.4285-3.1467 \quad 1.9360$

H $-0.1901-2.7644 \quad 1.4218$

H $-1.2277 \quad-4.8034 \quad 3.2480$

H $-1.4719-2.0349 \quad 3.2601$

H $-2.4476-3.1592 \quad 4.2291$

H $-3.9511-3.1103 \quad 2.5064$

H $-4.1947-2.4788-2.9477$

H $-4.1199-1.8764-1.3585$

$\begin{array}{llll}\mathrm{H} & -4.5440 & 0.4442 & -1.3262\end{array}$

H $-5.8233 \quad 1.0389-2.2575$

H $-6.3346 \quad 0.0457-4.0982$

H $-6.0968-2.9306-4.0518$

H $-5.5366-2.0211-5.4572$

H $-7.9226-1.1420-5.7670$

H $-8.4186-2.1505-4.3803$

H $-0.9132-0.5273-2.5509$

$\begin{array}{llll}\mathrm{H} & -2.1945 & -0.6187 & -1.3860\end{array}$

H $-0.8924 \quad 1.1012-4.1616$

$\begin{array}{llll}\mathrm{H} & -0.4264 & 2.3082 & -2.9546\end{array}$

H $-1.4531 \quad 3.4625-4.8550$

H $-2.4374 \quad 3.6262-3.4062$

$\begin{array}{llll}\mathrm{H} & -3.1371 & 2.1400 & -5.9443\end{array}$

H $-4.1163 \quad 4.3675 \quad-5.0274$

H $-5.9219 \quad 5.4204-4.2323$

H $-6.1780 \quad 3.8295-1.6229$

H $-6.6676 \quad 5.5201-1.7903$

H $-2.8470 \quad 4.7509-0.8674$

H $-3.7903 \quad 3.4030 \quad-1.3731$

H $8.4017 \quad 1.6729 \quad 0.6155$

H $8.4462 \quad 3.2635 \quad 1.3871$
H $\quad 6.9196 \quad 2.3724 \quad 1.2660$

H $6.90792 .5979-1.1806$

H $8.5068 \quad 3.3295 \quad-1.1821$

H $4.9156 \quad 3.9293 \quad-0.1669$

H $5.7728 \quad 4.4315 \quad 1.2929$

H $\quad \begin{array}{llll}5.1317 & 5.6492 & 0.1830\end{array}$

H $7.6507 \quad 5.3171 \quad-0.0105$

H $6.4918 \quad 6.3262 \quad-1.8831$

H $5.5652 \quad 3.6033 \quad-2.6008$

H $4.0315 \quad 4.0790-4.9548$

H $3.3692 \quad 3.5916 \quad-3.3949$

H $2.7574 \quad 6.2347 \quad-4.4927$

H $1.0887 \quad 7.3311 \quad-3.1161$

H $-1.2001 \quad 5.3221 \quad-2.8177$

H $-0.4283 \quad 6.2148 \quad-1.5108$

H $-2.5203 \quad 7.0552 \quad-2.5577$

H $8.5004-5.8114 \quad-1.0052$

H $\quad 8.1815 \quad-7.5240 \quad-1.3313$

H $9.7384-3.8938-6.6850$

H $8.0661-3.4425 \quad-6.3220$

H $\quad 9.3278-2.2069-6.2916$

H $9.1198-4.5440 \quad-4.2935$

H 10.4168 - $3.3507 \quad-4.3369$

H $\quad 0.5899 \quad 1.8103 \quad 4.6569$

H $-1.0436 \quad 2.3458 \quad 5.0134$

H $\quad 0.3073 \quad 3.4880 \quad 5.1530$

H $2.6695 \quad 1.6967 \quad 5.5481$

H $3.6555 \quad 1.6721 \quad 7.0187$

H $2.4751 \quad 0.3888 \quad 6.7110$

H $-0.0825 \quad 0.9909 \quad 6.9118$

$\begin{array}{llll}\mathrm{H} & -0.5356 & 2.6128 & 7.4078\end{array}$

$\begin{array}{llll}\mathrm{H} & 1.8319 & 3.3423 & 7.2438\end{array}$

$\begin{array}{llll}\mathrm{H} & 0.9164 & 2.5816 & 9.4243\end{array}$

H $2.6171 \quad 2.1059 \quad 9.2908$

H $1.6188 \quad-2.3013 \quad 10.2442$

H $-3.8020 \quad-2.6826 \quad 10.7453$

H $-2.2543-1.8361 \quad 10.6266$

H $-3.7552-0.9207 \quad 10.8162$

H $2.5849 \quad-2.7902 \quad-3.9223$

H $3.5839-2.7244 \quad-2.4637$

H $3.8564-4.0001-3.6456$

H $2.6150 \quad-4.8642 \quad-1.6643$

H $0.6798 \quad-5.8750 \quad-2.8445$

H $-0.2434 \quad-3.4149 \quad-3.4972$

H $-0.6541-3.8946-6.3932$

H $-2.4112-1.8127 \quad-4.1949$

H $-3.0137-3.1613-6.0721$

H $-2.5790 \quad-4.5104 \quad-4.9975$

H $\quad 0.5501 \quad-2.3167 \quad-7.4204$

H $\quad 0.3273 \quad-0.2654 \quad-8.6450$

H $-0.6132 \quad 0.4092 \quad-7.2858$

H $7.4653-0.6680 \quad-3.3052$

H $-7.3166 \quad 4.4060 \quad-3.8151$

H $-3.7651 \quad 1.5052-4.4221$

H $7.7146 \quad 5.2059 \quad-2.4949$

H $\quad 5.3845 \quad 2.9822 \quad 3.2132$

H $1.1952-2.6019-1.8968$

Н $1.6724-0.1910-1.9226$

H $\quad 0.5718 \quad-0.8273-1.0456$

H $-2.4826 \quad 11.6449-2.6345$
H $\quad-2.7106 \quad 8.8136 \quad 7.2549$

H $2.2047-6.0454 \quad-3.7268$

C $-0.5325 \quad-0.0168 \quad 1.2086$

$\begin{array}{llll}\mathrm{O} & -0.1766 & -1.0490 & 0.5313\end{array}$

$\begin{array}{llll}\mathrm{O} & -1.0214 & 0.0253 & 2.3421\end{array}$

H $2.4438 \quad-1.1139 \quad 9.2182$

$\begin{array}{llll}\mathrm{H} & 1.3399 & 0.9088 & 9.0427\end{array}$

H $1.1536 \quad 0.2768 \quad-7.1618$

H $-7.8151-2.9052 \quad-5.8692$

H $\quad 0.2044 \quad 6.6450 \quad-4.4714$

C $-3.1816-5.9560-0.4180$

H $-8.1655-6.4484-2.0982$

H $-9.5098 \quad-5.3620-2.1077$

H $-7.4318-2.5969-1.6293$

H $-8.8956-3.3248-1.0475$

H $-5.7628-3.9829-1.7324$

$\begin{array}{llll}\mathrm{H} & -6.2490 & -6.5620 & -3.0927\end{array}$

H $-5.7971-6.5619-1.3785$

H $-3.8701-6.9019-2.9517$

H -4.2068 -5.3486 -3.7034

H $-2.8725-6.9947 \quad-0.5930$

H $-4.1270-5.9730 \quad 0.1279$

H $-2.3134-5.1086-2.2057$

H $-3.6183-4.1825-1.5236$

H $-1.8572 \quad-1.8846 \quad 0.0351$

H $-3.1081-1.8870 \quad 0.9231$

H $-11.4852 \quad-5.6295 \quad 3.0741$

$\mathrm{H}-11.5734-4.2125 \quad 2.0073$

H -11.4386 $-5.8272 \quad 1.3134$

H $-9.1050 \quad-6.0348 \quad 2.3505$

H $-9.2780 \quad-4.4243 \quad 3.0084$

H -11.7472 $-4.6554-1.9662$

H $-10.7925-4.6110-0.7335$

H $-1.2905 \quad-5.0956 \quad 0.1310$

H $1.9131 \quad 0.0616 \quad 0.3818$

\section{TC}

$\begin{array}{llll}\mathrm{N} & -2.3915 & -5.1954 & 0.3908\end{array}$

C $-3.4257-5.1505-1.8390$

$\begin{array}{llll}\text { C } & -4.3902 & -5.8181 & -2.8281\end{array}$

$\begin{array}{llll}\text { C } & -5.8570 & -5.8764 & -2.3917\end{array}$

$\mathrm{N}-6.4127 \quad-4.5353-2.2395$

C $-7.7383-4.2892 \quad-2.1209$

$\mathrm{N}-8.6290 \quad-5.2496 \quad-2.3855$

$\begin{array}{llll}\mathrm{N} & -8.1791 & -3.0436 & -1.8686\end{array}$

$\begin{array}{llll}\mathrm{C}-11.2621 & -4.8289 & 2.1046\end{array}$

$\begin{array}{llll}\text { C } & -9.7318 & -4.8417 & 2.1144\end{array}$

C $-9.0627-4.2992 \quad 0.8466$

$\begin{array}{lllll}\text { O } & -7.9105 & -4.6644 & 0.5653\end{array}$

$\begin{array}{llll}\text { O } & -9.7650 & -3.4541 & 0.1435\end{array}$

O $-11.2424 \quad-4.7917 \quad-1.5768$

O $-2.9463 \quad-1.7367 \quad-0.0139$

C $7.9617-7.0317-0.6388$

C $6.5095-6.8124-0.2196$

O $5.5757 \quad-6.9295 \quad-1.0098$

N $6.2807 \quad-6.4753 \quad 1.0892$

$\begin{array}{llll}\text { C } & 4.9162 & -6.4385 & 1.6067\end{array}$

C $4.2290 \quad-5.0768 \quad 1.4172$

O 4.8488 -4.0169 1.5269 
C $5.1208-6.6910 \quad 3.1172$

C $6.4651 \quad-6.0029 \quad 3.3963$

C $7.2927 \quad-6.3208 \quad 2.1419$

$\begin{array}{llll}\mathrm{N} & 2.8911 & -5.1497 & 1.2184\end{array}$

C $\quad \begin{array}{llll}\text { C } & 1.9861 & -4.0190 & 1.3303\end{array}$

C $0.6316 \quad-4.5514 \quad 1.8046$

$\begin{array}{llll}\mathrm{O} & 0.5153 & -5.7073 & 2.2120\end{array}$

$\begin{array}{llll}\mathrm{N} & -0.4158 & -3.6850 & 1.7372\end{array}$

C $-1.6598-4.0033 \quad 2.4319$

$\begin{array}{llll}\text { C } & -2.7401 & -4.6993 & 1.5930\end{array}$

$\begin{array}{llll}\text { O } & -3.8810 & -4.8045 & 2.0626\end{array}$

$\begin{array}{llll}\text { C } & -2.2729 & -2.7717 & 3.1646\end{array}$

$\begin{array}{llll}\text { O } & -3.4600 & -2.2489 & 2.5640\end{array}$

$\begin{array}{llll}\text { C } 9.0016 & -3.5160 & -6.1175\end{array}$

$\begin{array}{llll}\text { C } & 9.4896 & -3.6952 & -4.6742\end{array}$

$\begin{array}{llll}\text { C } & 8.6999 & -2.8425 & -3.7044\end{array}$

O $8.6248-1.5732-4.1043$

$\begin{array}{llll}\text { O } & 8.1820 & -3.2716 & -2.6831\end{array}$

C $1.2821 \quad-5.3485 \quad-3.4550$

$\begin{array}{llll}\text { C } & 0.7506 & -4.6069 & -4.6829\end{array}$

$\begin{array}{llll}\text { O } & 1.1227 & -4.8589 & -5.8256\end{array}$

C $1.8685-4.4477-2.3623$

C $2.9581 \quad-3.5332-2.9168$

$\begin{array}{lllll}\mathrm{O} & 0.7955 & -3.7260 & -1.7381\end{array}$

N $-0.2157 \quad-3.6559-4.4423$

$\begin{array}{llll}\text { C } & -1.0258 & -3.1568 & -5.5380\end{array}$

C $-0.7656-1.6839-5.9179$

$\begin{array}{llll}\text { O } & -1.3414 & -0.7597 & -5.3160\end{array}$

$\begin{array}{llll}\text { C } & -2.5186 & -3.3097 & -5.2203\end{array}$

$\begin{array}{lllll}\text { O } & -2.8806 & -2.5683 & -4.0609\end{array}$

N $0.0278-1.4727 \quad-6.9867$

C $0.2466-0.1520-7.5806$

C $-7.7589-1.7503-5.2188$

C $-6.3365-1.7934-4.6491$

N $-6.0607 \quad-0.6464 \quad-3.7731$

C $-5.4014-0.6816-2.6085$

$\mathrm{N}-4.7985-1.8130 \quad-2.1898$

$\begin{array}{llll}\mathrm{N} & -5.4127 & 0.4041 & -1.8169\end{array}$

$\begin{array}{llll}\text { C } & -6.0760 & 4.7769 & -3.6409\end{array}$

$\begin{array}{llll}C & -5.4179 & 3.4807 & -4.0996\end{array}$

$\begin{array}{lllll}\text { O } & -5.8914 & 2.3859 & -3.7581\end{array}$

$\begin{array}{llll}\text { C } & -5.7928 & 4.9998 & -2.1378\end{array}$

C $-4.3847 \quad 5.4916-1.8034$

$\mathrm{N}-3.5365 \quad 4.6006-1.2804$

$\begin{array}{lllll}\mathrm{O} & -4.0892 & 6.6959 & -1.9750\end{array}$

$\begin{array}{llll}\mathrm{N} & -4.2806 & 3.5979 & -4.8192\end{array}$

$\begin{array}{llll}\text { C } & -3.2624 & 2.5405 & -4.8991\end{array}$

C $-2.0010 \quad 3.0023 \quad-4.1362$

$\begin{array}{llll}C & -1.2539 & 1.8874 & -3.4100\end{array}$

C $-2.1190 \quad 1.2240 \quad-2.3552$

$\begin{array}{llll}\mathrm{N} & -1.9476 & -0.0987 & -2.2036\end{array}$

$\begin{array}{lllll}\text { O } & -2.9701 & 1.8432 & -1.6804\end{array}$

$\begin{array}{lllll}\text { O } & 0.7593 & -1.0776 & -1.9718\end{array}$

$\begin{array}{llll}\text { C } & -2.7705 & 8.1870 & 6.5219\end{array}$

$\begin{array}{lllll}\mathrm{C} & -1.9668 & 6.8868 & 6.6292\end{array}$

N $-2.3892 \quad 5.8552 \quad 5.6779$

$\begin{array}{llll}\text { C } & -2.0189 & 5.8011 & 4.3857\end{array}$

$\mathrm{N}-1.2553 \quad 6.7504 \quad 3.8451$

$\begin{array}{llll}\mathrm{N} & -2.4376 & 4.7845 & 3.6224\end{array}$

C $-1.2904 \quad 11.8859-1.8135$ $\begin{array}{llll}\text { C } & -0.7328 & 10.5377 & -1.3874\end{array}$

N $-1.7784 \quad 9.7473 \quad-0.7375$

C $-1.5329 \quad 8.6328 \quad-0.0252$

$\begin{array}{llll}\mathrm{N} & -2.5324 & 7.7832 & 0.2420\end{array}$

$\begin{array}{llll}\mathrm{N} & -0.3251 & 8.4232 & 0.5031\end{array}$

C $\quad 0.7413 \quad 6.4577 \quad-3.4108$

C $1.5585 \quad 5.1729-3.3638$

$\begin{array}{lllll}\text { O } & 1.2225 & 4.1947 & -2.6913\end{array}$

C $-0.5434 \quad 6.3243 \quad-2.5976$

$\begin{array}{lllll}\mathrm{O} & -1.3573 & 7.4773 & -2.7874\end{array}$

N $2.6700 \quad 5.1978 \quad-4.1467$

C $3.7731 \quad 4.2886-3.9080$

$\begin{array}{llll}\text { C } & 4.9287 & 5.1109 & -3.3199\end{array}$

O $5.0081 \quad 6.3166 \quad-3.5640$

N $5.8003 \quad 4.4187 \quad-2.5643$

C $6.9557 \quad 5.0511-1.9366$

C $7.1711 \quad 4.5424 \quad-0.4988$

C $7.6179 \quad 3.0675 \quad-0.4796$

C $5.9279 \quad 4.7817 \quad 0.3690$

C $7.9803 \quad 2.5266 \quad 0.9052$

$\begin{array}{llll}\text { C } & 1.7134 & 1.8283 & 8.8593\end{array}$

$\begin{array}{llll}\text { C } & 1.4449 & 2.2088 & 7.3942\end{array}$

$\begin{array}{llll}\text { C } & -0.0060 & 1.8669 & 7.0082\end{array}$

$\begin{array}{llll}\text { C } & 2.4665 & 1.5367 & 6.4675\end{array}$

$\begin{array}{llll}\text { C } & -0.4299 & 2.3367 & 5.6124\end{array}$

C $1.5910-1.9623 \quad 9.2162$

C $1.6592 \quad-3.0001 \quad 8.0803$

$\begin{array}{llll}\text { C } & 1.7097 & -2.3977 & 6.6904\end{array}$

$\begin{array}{llll}\text { C } & 0.5762 & -1.7829 & 6.1388\end{array}$

C $2.8753 \quad-2.4455 \quad 5.9156$

C $0.6051-1.2282 \quad 4.8595$

C $2.9084-1.9037 \quad 4.6273$

C $1.7727-1.2932 \quad 4.0950$

C $-3.3382 \quad-1.7601 \quad 10.3063$

C $-3.5151-1.8304 \quad 8.7862$

C $-2.8499-0.6869 \quad 7.9992$

$\begin{array}{llll}\text { C } & -2.9808 & -0.9270 & 6.4901\end{array}$

$\begin{array}{llll}\text { C } & -3.4178 & 0.6874 & 8.3753\end{array}$

$\begin{array}{llll}C & -0.6388 & 2.6261 & 1.0900\end{array}$

$\begin{array}{llll}\text { C } & -0.4759 & 5.1148 & 1.0922\end{array}$

$\begin{array}{lllll}\mathrm{O} & 0.4047 & 1.3713 & -0.6417\end{array}$

$\begin{array}{llll}\mathrm{O} & -1.7423 & 5.2398 & 0.9422\end{array}$

$\begin{array}{llll}\mathrm{O} & 0.2356 & 6.0001 & 1.6554\end{array}$

C $\quad 6.8078 \quad 1.6474 \quad-4.0737$

C $6.0485 \quad-0.8246 \quad 2.2134$

C $4.2863-1.4716-0.0260$

C $6.1422-1.1119-1.7555$

C $5.0187-0.7030-1.0835$

C $4.5192 \quad 0.5843 \quad-1.4074$

N $3.4089 \quad 1.0206 \quad-0.7642$

$\begin{array}{llll}\mathrm{N} & 5.0926 & 1.3537 & -2.3563\end{array}$

C $6.1575 \quad 0.8305 \quad-2.9919$

N $6.7120 \quad-0.3508 \quad-2.7174$

$\begin{array}{llll}\text { S } & 2.8839 & 1.7163 & 1.8477\end{array}$

$\begin{array}{llll}C & 2.9555 & 0.4443 & 0.5353\end{array}$

N $3.8620 \quad-0.5559 \quad 1.0372$

C $4.8284-0.0106 \quad 1.9265$

C $4.4759 \quad 1.1661 \quad 2.4748$

C $5.1344 \quad 1.9767 \quad 3.5406$

$\begin{array}{llll}\text { C } & -0.1951 & 1.3584 & 0.4219\end{array}$ $\begin{array}{llll}\text { C } & 0.1604 & 3.8387 & 0.6092\end{array}$

H $3.0582 \quad 1.9416-1.0007$

H $-1.7009 \quad 2.7465 \quad 0.8372$

$\begin{array}{llll}\mathrm{H} & -0.5934 & 2.4875 & 2.1729\end{array}$

H $7.5155 \quad 2.3605 \quad-3.6374$

H $\quad 6.0646 \quad 2.2176-4.6333$

H $7.3626 \quad 0.9956 \quad-4.7501$

H $5.7747-1.8577 \quad 2.4512$

H $6.6081 \quad-0.4025 \quad 3.0485$

H $6.7149-0.8613 \quad 1.3435$

H $4.5057 \quad 2.0398 \quad 4.4363$

$\begin{array}{lllll}\mathrm{H} & 0.1934 & 3.8537 & -0.4869\end{array}$

H $6.6204-2.0729-1.5788$

H $3.3670-1.9016 \quad-0.4389$

H $\quad \begin{array}{llll}6.0873 & 1.5303 & 3.8342\end{array}$

$\begin{array}{llll}\mathrm{H} & 1.1920 & 3.7955 & 0.9608\end{array}$

H $4.8565 \quad-2.3022 \quad 0.3842$

H $-0.2723 \quad-0.7421 \quad 4.4480$

H $\quad \begin{array}{llll}1.8007 & -0.8667 & 3.0988\end{array}$

H $-0.3402 \quad-1.7470 \quad 6.7196$

$\begin{array}{llll}\text { H } & 3.7637 & -2.9220 & 6.3222\end{array}$

H $3.8158-1.9632 \quad 4.0346$

H $\quad 0.7852 \quad-3.6594 \quad 8.1518$

H $2.5379 \quad-3.6375 \quad 8.2275$

H $\quad 0.7005 \quad-1.3334 \quad 9.1172$

H $-3.1055-2.7816 \quad 8.4225$

H $-4.5862-1.8494 \quad 8.5407$

$\begin{array}{llll}\mathrm{H} & -4.4982 & 0.7224 & 8.1887\end{array}$

H $-3.2524 \quad 0.9250 \quad 9.4299$

H $-2.9482 \quad 1.4776 \quad 7.7794$

H $-1.7790 \quad-0.6858 \quad 8.2549$

$\begin{array}{llll}\mathrm{H} & -4.0317 & -0.9041 & 6.1796\end{array}$

H $-2.4454-0.1670 \quad 5.9112$

H $-2.5776-1.9037 \quad 6.2057$

H $-2.7061 \quad 3.9101 \quad 4.0468$

H $-2.2941 \quad 4.8307 \quad 2.6046$

H $-1.0296 \quad 7.5738 \quad 4.3774$

H $-0.7036 \quad 6.5446 \quad 2.9873$

H $-3.1395 \quad 5.2403 \quad 5.9573$

H $-0.8961 \quad 7.0632 \quad 6.4831$

H $-2.0691 \quad 6.4541 \quad 7.6268$

H $-2.6854 \quad 8.6391 \quad 5.5289$

H $\quad-3.8311 \quad 8.0045 \quad 6.7143$

$\mathrm{H}-2.2715 \quad 6.8855 \quad 0.6814$

$\mathrm{H}-3.2632 \quad 7.6804-0.4617$

H $-2.6850 \quad 9.7780 \quad-1.1839$

H $\quad 0.4279 \quad 9.0575 \quad 0.3016$

$\mathrm{H} \quad-0.0990 \quad 7.5224 \quad 0.9574$

H $-0.3287 \quad 9.9755 \quad-2.2377$

H $\quad 0.0751 \quad 10.7110 \quad-0.6701$

H $-0.5099 \quad 12.4797 \quad-2.2950$

H $-1.6696 \quad 12.4425 \quad-0.9526$

H $\quad 8.5673 \quad-7.5055 \quad 0.1367$

H $7.8628 \quad-7.2502 \quad 2.2719$

H $7.9985 \quad-5.5218 \quad 1.8964$

H $6.9472 \quad-6.3574 \quad 4.3104$

H $6.3140 \quad-4.9239 \quad 3.4734$

H $5.1917 \quad-7.7693 \quad 3.2932$

H $4.2992-6.3015 \quad 3.7235$

H 4.3265 -7.2162 1.1153 
$\begin{array}{llll}\text { H } & 2.4284 & -6.0445 & 1.3414\end{array}$

H $1.8469 \quad-3.5102 \quad 0.3721$

$\begin{array}{llll}\text { H } & 2.3741 & -3.2914 & 2.0522\end{array}$

$\begin{array}{llll}\mathrm{H} & -0.2705 & -2.7318 & 1.3907\end{array}$

H $-1.3952-4.7546 \quad 3.1837$

H $-1.5527-1.9569 \quad 3.1694$

H $-2.4928-3.0534 \quad 4.1989$

$\begin{array}{llll}\mathrm{H} & -4.0621 & -3.0203 & 2.5065\end{array}$

$\begin{array}{llll}\mathrm{H} & -4.3147 & -2.3373 & -2.9257\end{array}$

H $-4.2316-1.7489-1.3339$

$\begin{array}{llll}\mathrm{H} & -4.5676 & 0.5887 & -1.2790\end{array}$

$\begin{array}{llll}\mathrm{H} & -5.7864 & 1.2473 & -2.2472\end{array}$

$\begin{array}{llll}\mathrm{H} & -6.3141 & 0.2821 & -4.0983\end{array}$

H $-6.2065-2.7022-4.0594$

H $-5.5930-1.8140 \quad-5.4551$

$\begin{array}{llll}\mathrm{H} & -7.9367 & -0.8299 & -5.7849\end{array}$

H $-8.4892-1.8245-4.4099$

H $-1.0766-0.5183-2.5269$

H $-2.3573-0.5130-1.3678$

H $-0.8967 \quad 1.1278-4.1090$

$\begin{array}{llll}\mathrm{H} & -0.3828 & 2.3162 & -2.9001\end{array}$

$\begin{array}{llll}\mathrm{H} & -1.3122 & 3.5149 & -4.8158\end{array}$

$\begin{array}{llll}\mathrm{H} & -2.3163 & 3.7314 & -3.3875\end{array}$

H -3.04462 .3028 -5.9441

H $-3.9637 \quad 4.5380 \quad-5.0142$

H $-5.7336 \quad 5.6429-4.2160$

H $-6.0185 \quad 4.0790-1.5937$

H $-6.4658 \quad 5.7797-1.7724$

H $-2.6715 \quad 4.9074-0.8405$

H $-3.6630 \quad 3.5910-1.3321$

$\begin{array}{llll}\mathrm{H} & 8.3714 & 1.5072 & 0.8301\end{array}$

$\begin{array}{llll}\mathrm{H} & 8.7462 & 3.1446 & 1.3867\end{array}$

H $7.1088 \quad 2.4913 \quad 1.5634$

$\begin{array}{lllll}\text { H } & 6.8207 & 2.4415 & -0.8942\end{array}$

H $8.48292 .9548 \quad-1.1464$

H $5.1038 \quad 4.1220 \quad 0.0772$

H $\quad 6.1401 \quad 4.5942 \quad 1.4247$

H $\quad \begin{array}{llll}5.5773 & 5.8150 & 0.2773\end{array}$

H $7.9967 \quad 5.1442 \quad-0.0922$

H $6.7677 \quad 6.1264-1.9458$

H $5.5935 \quad 3.4377 \quad-2.3891$

H $4.1077 \quad 3.8304 \quad-4.8469$

H $3.4476 \quad 3.4875 \quad-3.2449$

$\begin{array}{llll}\text { H } & 3.0027 & 6.1151 & -4.4312\end{array}$

$\begin{array}{lllll}\text { H } & 1.3524 & 7.2869 & -3.0336\end{array}$

H $-1.0666 \quad 5.4170-2.9157$

H $-0.2897 \quad 6.1876-1.5374$

H $-2.2732 \quad 7.2200-2.5728$

H $8.4138 \quad-6.0659-0.8938$

H $7.9540 \quad-7.6501-1.5364$

H $9.5751 \quad-4.1504-6.7999$

H $7.9466-3.7919-6.2108$

H $9.1099 \quad-2.4769-6.4330$

$\begin{array}{llll}\text { H } & 9.4087 & -4.7329 & -4.3440\end{array}$

H $10.5443 \quad-3.4018$-4.6016

H $\quad 0.1278 \quad 1.8264 \quad 4.8224$

H $-1.4919 \quad 2.1213 \quad 5.4385$

H $-0.2732 \quad 3.4167 \quad 5.4936$

H $2.3146 \quad 1.8127 \quad 5.4200$

H $3.4860 \quad 1.8255 \quad 6.7461$
H $2.3968 \quad 0.4472 \quad 6.5301$

$\mathrm{H}-0.1414 \quad 0.7814 \quad 7.0754$

H $-0.6782 \quad 2.3071 \quad 7.7566$

H $\quad \begin{array}{llll}1.5642 & 3.3001 & 7.2995\end{array}$

H $1.0194 \quad 2.3362 \quad 9.5381$

H $2.7322 \quad 2.0966 \quad 9.1577$

H $1.5480 \quad-2.455510 .1919$

H $-3.7712-2.6400 \quad 10.7912$

H $-2.2767-1.7199 \quad 10.5773$

H $-3.8236 \quad-0.8777 \quad 10.7343$

H $2.5488-2.8414 \quad-3.6602$

H $3.4258-2.9446-2.1275$

H $3.7405-4.1301-3.3923$

H $2.3141-5.1008$ - 1.6016

H $\quad 0.4633 \quad-5.9318 \quad-3.0182$

H $-0.4730 \quad-3.4614 \quad-3.4809$

H $-0.7802-3.7883-6.3966$

H $-2.5213-1.6668-4.2134$

H $-3.1126 \quad-2.9981-6.0922$

H $-2.7315-4.3613 \quad-5.0159$

H $\quad 0.5283 \quad-2.2703 \quad-7.3569$

H $\quad 0.2389-0.2371 \quad-8.6690$

H $-0.5641 \quad 0.4987 \quad-7.2534$

H $7.9742-1.0696-3.5142$

H $-7.1534 \quad 4.6710 \quad-3.7843$

H $-3.7083 \quad 1.6558$-4.4442

H $7.8585 \quad 4.8692 \quad-2.5353$

H $5.3309 \quad 3.0009 \quad 3.2025$

H $0.9520 \quad-2.7634 \quad-1.8548$

H $1.3591-0.3350 \quad-2.1230$

H $0.4204-0.9328-1.0440$

H $-2.1051 \quad 11.7668$-2.5359

H $-2.4100 \quad 8.9106 \quad 7.2580$

$\begin{array}{llll}\text { H } & 2.0402 & -6.0449 & -3.8177\end{array}$

$\begin{array}{llll}\text { C } & -0.5396 & 0.0172 & 1.1380\end{array}$

$\begin{array}{llll}\mathrm{O} & -0.2525 & -1.0471 & 0.4751\end{array}$

$\begin{array}{llll}\mathrm{O} & -1.0494 & 0.0982 & 2.2589\end{array}$

H $2.4689-1.3102 \quad 9.2002$

H $1.5965 \quad 0.7525 \quad 9.0056$

H $1.1998 \quad 0.2805 \quad-7.2597$

H $-7.9027 \quad-2.5956-5.8969$

H $\quad 0.4916 \quad 6.7009-4.4496$

C $-3.3681-5.8424 \quad-0.4762$

H $-8.3509-6.2008-2.1989$

H $-9.6320-5.0450 \quad-2.2109$

H $-7.4255-2.4081-1.6348$

H $-8.9264-3.0825-1.0680$

H $-5.8365-3.8735-1.7356$

H $-6.4299-6.3925 \quad-3.1678$

H $-5.9701-6.4414-1.4569$

H $-4.0606-6.8478-3.0102$

H $-4.3414-5.2963-3.7902$

H $-3.1151-6.9039-0.5980$

H $-4.3224 \quad-5.7844 \quad 0.0516$

H $-2.4239-5.1421 \quad-2.2867$

H $-3.6722-4.0981-1.6758$

H $-1.9727-1.7817 \quad 0.0512$

H $-3.2297-1.7778 \quad 0.9369$

H $-11.6640-5.23513 .0382$

H -11.6364 -3.8100 1.9781
H -11.6558 $-5.4269 \quad 1.2763$

H $-9.3381 \quad-5.8494 \quad 2.2795$

H $-9.3571-4.2340 \quad 2.9496$

H -11.8180 -4.1909 -2.0652

H - $10.8877 \quad-4.2549-0.8175$

$\begin{array}{llll}\mathrm{H} & -1.4530 & -5.0187 & 0.0557\end{array}$

H $1.9720 \quad 0.0109 \quad 0.3878$

IP

$\begin{array}{llll}\mathrm{N} & -2.4798 & -5.1904 & 0.1883\end{array}$

C $-3.5416-4.9567-2.0182$

C $-4.5186-5.5424-3.0460$

C $-5.9820-5.6206-2.6022$

N $-6.5290 \quad-4.2877-2.3693$

C $-7.8498-4.0427 \quad-2.2131$

N $-8.7488-4.9888-2.4976$

$\mathrm{N}-8.2808-2.8065-1.9016$

C - $11.3091 \quad-4.8072 \quad 2.0121$

$\begin{array}{llll}\text { C } & -9.7790 & -4.7821 & 2.0210\end{array}$

C $-9.1258-4.1696 \quad 0.7767$

$\begin{array}{lllll}\text { O } & -7.9689 & -4.5021 & 0.4744\end{array}$

$\begin{array}{lllll}\text { O } & -9.8447 & -3.3039 & 0.1177\end{array}$

O $-11.3470-4.5720-1.6299$

O $-2.9420-1.6955 \quad-0.0811$

C $7.8513-7.0736-1.0983$

$\begin{array}{llll}\text { C } & 6.4369 & -6.9271 & -0.5461\end{array}$

$\begin{array}{llll}\text { O } & 5.4460 & -7.3448 & -1.1378\end{array}$

N $6.3076 \quad-6.2962 \quad 0.6675$

$\begin{array}{llll}\text { C } & 5.0152 & -6.2527 & 1.3357\end{array}$

$\begin{array}{llll}\text { C } & 4.1544 & -5.0497 & 0.9222\end{array}$

$\begin{array}{llll}\text { O } & 4.6563 & -3.9777 & 0.5646\end{array}$

C $5.4079-6.1040 \quad 2.8240$

$\begin{array}{llll}\text { C } & 6.6907 & -5.2611 & 2.7591\end{array}$

$\begin{array}{llll}\text { C } & 7.3992 & -5.7702 & 1.4933\end{array}$

$\begin{array}{llll}\text { N } & 2.8278 & -5.2164 & 1.0886\end{array}$

$\begin{array}{llll}\text { C } & 1.8744 & -4.1235 & 1.0717\end{array}$

$\begin{array}{llll}\text { C } & 0.5640 & -4.6422 & 1.6634\end{array}$

$\begin{array}{llll}\text { O } & 0.4964 & -5.7630 & 2.1674\end{array}$

N $-0.4937 \quad-3.7930 \quad 1.5766$

C $-1.7340-4.1032 \quad 2.2743$

$\begin{array}{llll}\text { C } & -2.8297 & -4.7370 & 1.4069\end{array}$

$\begin{array}{llll}\text { O } & -3.9750 & -4.8350 & 1.8669\end{array}$

$\begin{array}{llll}\text { C } & -2.3160 & -2.8880 & 3.0581\end{array}$

$\begin{array}{llll}\text { O } & -3.4864 & -2.3074 & 2.4758\end{array}$

C $8.8661-3.2712-6.3868$

C $9.0517-3.5892-4.8984$

C $8.3179-2.6150 \quad-3.9664$

O $8.3194-1.3837-4.3348$

O $7.7912 \quad-3.0587 \quad-2.9212$

C $1.1583 \quad-5.1608-3.7301$

C $0.6805 \quad-4.2961 \quad-4.8991$

O $1.0834-4.4438-6.0495$

C $1.7358-4.4054-2.5272$

$\begin{array}{llll}\text { C } & 2.9688 & -3.5824 & -2.8836\end{array}$

O $0.7033-3.6125 \quad-1.9155$

N $-0.2988-3.3754 \quad-4.6029$

C $-1.1255-2.8370 \quad-5.6673$

C $-0.8917-1.3460-5.9882$

O $-1.5197 \quad-0.4569-5.3846$ 
$\begin{array}{llll}\text { C } & -2.6101 & -3.0237 & -5.3334\end{array}$

$\begin{array}{llll}\text { O } & -2.9517 & -2.3435 & -4.1302\end{array}$

$\begin{array}{llll}\mathrm{N} & -0.0635 & -1.0795 & -7.0147\end{array}$

$\begin{array}{llll}\text { C } & 0.1352 & 0.2686 & -7.5477\end{array}$

$\begin{array}{llll}\text { C } & -7.8594 & -1.3669 & -5.1744\end{array}$

C $-6.4263-1.4564-4.6407$

$\begin{array}{llll}\mathrm{N} & -6.1203 & -0.3548 & -3.7188\end{array}$

$\begin{array}{llll}\mathrm{C} & -5.4328 & -0.4513 & -2.5750\end{array}$

$\begin{array}{llll}\mathrm{N} & -4.8259 & -1.6035 & -2.2277\end{array}$

$\begin{array}{llll}\mathrm{N} & -5.4165 & 0.5934 & -1.7277\end{array}$

$\begin{array}{lllll}\mathrm{C} & -6.0782 & 5.0430 & -3.2595\end{array}$

$\begin{array}{llll}\text { C } & -5.4472 & 3.7741 & -3.8203\end{array}$

$\begin{array}{llll}\text { O } & -5.9510 & 2.6673 & -3.5722\end{array}$

$\begin{array}{llll}C & -5.7684 & 5.1372 & -1.7466\end{array}$

$\begin{array}{llll}\mathrm{C} & -4.3441 & 5.5720 & -1.4052\end{array}$

$\begin{array}{llll}\mathrm{N} & -3.4943 & 4.6273 & -0.9860\end{array}$

$\begin{array}{lllll}\mathrm{O} & -4.0336 & 6.7823 & -1.4767\end{array}$

$\begin{array}{llll}\mathrm{N} & -4.2942 & 3.9229 & -4.5057\end{array}$

$\begin{array}{llll}\text { C } & -3.3076 & 2.8479 & -4.6760\end{array}$

$\begin{array}{lllll}C & -2.0086 & 3.2333 & -3.9421\end{array}$

C $-1.24412 .0566-3.3426$

C $-2.0768 \quad 1.3163-2.3155$

$\mathrm{N}-1.8781-0.0095 \quad-2.2420$

$\begin{array}{lllll}\text { O } & -2.9348 & 1.8816 & -1.6007\end{array}$

$\begin{array}{lllll}\text { O } & 0.8985 & -0.9160 & -2.0649\end{array}$

$\begin{array}{llll}C & -2.6066 & 7.8456 & 7.0329\end{array}$

$\begin{array}{llll}\text { C } & -2.0013 & 6.4400 & 6.9914\end{array}$

$\begin{array}{llll}\mathrm{N} & -2.5084 & 5.6133 & 5.8924\end{array}$

$\begin{array}{llll}\mathrm{C} & -2.0418 & 5.6293 & 4.6300\end{array}$

$\begin{array}{llll}\mathrm{N} & -1.0966 & 6.4901 & 4.2546\end{array}$

$\begin{array}{llll}\mathrm{N} & -2.5432 & 4.7707 & 3.7349\end{array}$

C $-1.1847 \quad 11.9845-1.1035$

$\begin{array}{llll}C & -0.5907 & 10.6207 & -0.7769\end{array}$

$\begin{array}{llll}\mathrm{N} & -1.6114 & 9.7554 & -0.1853\end{array}$

$\begin{array}{llll}\mathrm{C} & -1.3445 & 8.5929 & 0.4353\end{array}$

$\begin{array}{llll}\mathrm{N} & -2.3455 & 7.7457 & 0.7159\end{array}$

$\begin{array}{llll}\mathrm{N} & -0.1135 & 8.3263 & 0.8770\end{array}$

$\begin{array}{lllll}\text { C } & 0.7611 & 6.6303 & -3.0251\end{array}$

C $1.6083 \quad 5.3746 \quad-3.1940$

$\begin{array}{lllll}\text { O } & 1.3074 & 4.2947 & -2.6802\end{array}$

$\begin{array}{llll}\text { C } & -0.6177 & 6.3255 & -2.4579\end{array}$

$\begin{array}{lllll}\text { O } & -1.3386 & 7.5422 & -2.2931\end{array}$

$\begin{array}{llll}\mathrm{N} & 2.7140 & 5.5695 & -3.9642\end{array}$

C $3.8600 \quad 4.6866 \quad-3.8868$

C $4.94895 .3889 \quad-3.0621$

$\begin{array}{lllll}\text { O } & 4.9105 & 6.6101 & -2.9017\end{array}$

N $5.9177 \quad 4.5769 \quad-2.5883$

C $6.9759 \quad 5.0735-1.7118$

$\begin{array}{llll}C & 6.8555 & 4.5176 & -0.2797\end{array}$

$\begin{array}{llll}\text { C } & 7.1834 & 3.0132 & -0.2314\end{array}$

C $5.4749 \quad 4.8289 \quad 0.3157$

C $7.2923 \quad 2.4426 \quad 1.1843$

C $1.8283 \quad 1.3161 \quad 8.9561$

C $\quad 1.6710 \quad 1.6609 \quad 7.4661$

C $0.2486 \quad 1.3285 \quad 6.9812$

$\begin{array}{llll}\text { C } & 2.7449 & 0.9575 & 6.6261\end{array}$

$\begin{array}{llll}\text { C } & -0.0719 & 1.7769 & 5.5512\end{array}$

C $1.6644 \quad-2.4868 \quad 9.1041$

$\begin{array}{llll}\text { C } & 1.8828 & -3.5436 & 8.0065\end{array}$

C $2.0161-2.9565 \quad 6.6168$
C $\quad 0.9121 \quad-2.3572 \quad 5.9936$

C $3.2288-2.9946 \quad 5.9171$

C $1.0111 \quad-1.8063 \quad 4.7177$

C $3.3354-2.4546 \quad 4.6315$

C $2.2269-1.8555 \quad 4.0322$

$\begin{array}{llll}\text { C } & -3.2482 & -2.2892 & 10.2676\end{array}$

C $-3.3496-2.3466 \quad 8.7409$

C $-2.6185-1.2145 \quad 7.9971$

$\begin{array}{llll}\text { C } & -2.6779 & -1.4460 & 6.4826\end{array}$

$\begin{array}{llll}\text { C } & -3.1717 & 0.1704 & 8.3542\end{array}$

$\begin{array}{llll}\text { C } & -0.4757 & 2.4847 & 1.4191\end{array}$

$\begin{array}{llll}\text { C } & -0.3002 & 4.9821 & 1.3994\end{array}$

$\begin{array}{lllll}\text { O } & 0.1893 & 1.3534 & -0.5614\end{array}$

$\begin{array}{llll}\text { O } & -1.5502 & 5.1342 & 1.1624\end{array}$

$\begin{array}{lllll}\mathrm{O} & 0.3891 & 5.8396 & 2.0260\end{array}$

C $7.0183 \quad 1.7448-4.3215$

$\begin{array}{llll}\text { C } & 6.0209 & -1.3906 & 1.6330\end{array}$

C $3.9168-1.1241-0.4950$

C $5.8546-0.9141-2.0575$

C $4.6902-0.4132-1.5565$

C $4.1530 \quad 0.8123 \quad-2.1326$

N $2.9218 \quad 1.1610 \quad-1.8620$

N $4.9925 \quad 1.5606 \quad-2.9592$

C $6.1107 \quad 1.0030 \quad-3.3820$

$\begin{array}{llll}\mathrm{N} & 6.5362 & -0.2224 & -3.0055\end{array}$

$\begin{array}{llll}\text { S } & 3.2159 & 1.3923 & 2.4661\end{array}$

$\begin{array}{llll}\text { C } & 3.0019 & 0.6058 & 0.9747\end{array}$

$\begin{array}{llll}\text { N } & 3.9202 & -0.3237 & 0.7701\end{array}$

$\begin{array}{llll}\text { C } & 4.8795 & -0.4429 & 1.7820\end{array}$

$\begin{array}{llll}C & 4.6318 & 0.4212 & 2.8114\end{array}$

C $5.3663 \quad 0.5695 \quad 4.1052$

$\begin{array}{llll}\text { C } & -0.1860 & 1.2589 & 0.5927\end{array}$

$\begin{array}{llll}\text { C } & 0.3349 & 3.6946 & 0.9364\end{array}$

H $2.66862 .0432 \quad-2.3087$

H $-1.5467 \quad 2.6856 \quad 1.2790$

H $-0.3298 \quad 2.25992 .4776$

H $7.80292 .2594 \quad-3.7545$

H $\quad 6.4560 \quad 2.4870 \quad-4.8882$

H $7.5153 \quad 1.0393 \quad-4.9918$

H $5.6717 \quad-2.4103 \quad 1.4477$

H $6.6361 \quad-1.3802 \quad 2.5329$

H $6.6460 \quad-1.0998 \quad 0.7823$

H $4.9192 \quad-0.0688 \quad 4.8741$

H $\quad 0.3644 \quad 3.6917 \quad-0.1602$

H $6.3059-1.8789-1.8223$

H $\quad 2.8673 \quad-1.2412 \quad-0.7636$

H $\quad 6.4133 \quad 0.2854 \quad 3.9822$

H $1.3668 \quad 3.6547 \quad 1.2931$

H $4.3201-2.1060 \quad-0.2531$

H $\quad 0.1574 \quad-1.3380 \quad 4.2431$

H $2.2953-1.4294 \quad 3.0372$

H $-0.0410-2.3304 \quad 6.5120$

H $4.0945 \quad-3.4621 \quad 6.3788$

H $4.2800 \quad-2.5124 \quad 4.0974$

H $1.0404-4.2463 \quad 8.0175$

H $2.7772-4.1309 \quad 8.2402$

H $\quad 0.7612-1.9005 \quad 8.9080$

$\begin{array}{llll}\mathrm{H} & -2.9445 & -3.3052 & 8.3921\end{array}$

H $-4.4067 \quad-2.3389 \quad 8.4409$

$\begin{array}{llll}\mathrm{H} & -4.2372 & 0.2359 & 8.1019\end{array}$
H $\quad-3.0660 \quad 0.3908 \quad 9.4202$

H $-2.6447 \quad 0.9540 \quad 7.7996$

H $-1.5616 \quad-1.2423 \quad 8.3049$

H $-3.7100-1.3963 \quad 6.1170$

H $-2.0936-0.7010 \quad 5.9321$

H $-2.2883-2.4352 \quad 6.2231$

H $-3.0224 \quad 3.9409 \quad 4.0472$

$\begin{array}{llll}\mathrm{H} & -2.2824 & 4.8547 & 2.7405\end{array}$

$\mathrm{H}-0.8100 \quad 7.2238 \quad 4.8803$

$\begin{array}{llll}\mathrm{H} & -0.5439 & 6.3261 & 3.3893\end{array}$

$\begin{array}{llll}\mathrm{H} & -3.3683 & 5.1084 & 6.0491\end{array}$

$\begin{array}{llll}\mathrm{H} & -0.9101 & 6.4740 & 6.9117\end{array}$

$\mathrm{H}-2.2207 \quad 5.9014 \quad 7.9162$

H $-2.4000 \quad 8.4053 \quad 6.1154$

H $\quad-3.6919 \quad 7.7981 \quad 7.1568$

H $-2.0617 \quad 6.8042 \quad 1.0295$

H $-3.1209 \quad 7.7090 \quad 0.0556$

H $-2.5317 \quad 9.8206 \quad-0.5984$

$\begin{array}{llll}\mathrm{H} & 0.6493 & 8.9407 & 0.6513\end{array}$

H $\quad 0.1001 \quad 7.4168 \quad 1.3134$

H $-0.1728 \quad 10.1382-1.6690$

H $\quad 0.2145 \quad 10.7581 \quad-0.0479$

H $-0.4196 \quad 12.6300-1.5408$

H $-1.5741 \quad 12.4672-0.2035$

$\begin{array}{llll}\text { H } & 8.5717 & -7.3818 & -0.3362\end{array}$

H $8.1169-6.5634 \quad 1.7400$

H $7.9409 \quad-4.9750 \quad 0.9721$

H $7.3144 \quad-5.3653 \quad 3.6503$

H $6.4360 \quad-4.20512 .6440$

H $5.6178 \quad-7.0953 \quad 3.2377$

H $4.6182-5.6455 \quad 3.4252$

H $4.4656 \quad-7.1745 \quad 1.1278$

$\begin{array}{llll}\mathrm{H} & 2.4524 & -6.0778 & 1.4745\end{array}$

H $\quad 1.6855 \quad-3.7460 \quad 0.0627$

H $\quad 2.2345 \quad-3.2905 \quad 1.6862$

H $-0.3504 \quad-2.8454 \quad 1.2077$

H $-1.4782-4.88812 .9940$

H $-1.5718-2.0938 \quad 3.1015$

H $-2.5500 \quad-3.2087 \quad 4.0773$

H $-4.1087 \quad-3.0597 \quad 2.3829$

H $-4.3681-2.1021 \quad-2.9980$

H $-4.2393-1.5847-1.3830$

H $-4.5338 \quad 0.7585-1.2410$

H $-5.8045 \quad 1.4527 \quad-2.1114$

H $-6.3721 \quad 0.5903 \quad-3.9942$

H $-6.2967-2.3930-4.0958$

H $-5.7001-1.4483-5.4625$

H $-8.0352-0.4189 \quad-5.6935$

H $-8.5723-1.4672 \quad-4.3528$

H $-0.9897-0.3831 \quad-2.5782$

H $-2.2734-0.4660 \quad-1.4241$

H $-0.9228 \quad 1.3523 \quad-4.1130$

H $-0.3488 \quad 2.4336-2.8350$

H $-1.3441 \quad 3.7897-4.6111$

H $-2.2806 \quad 3.9029-3.1248$

H $-3.1341 \quad 2.6537 \quad-5.7385$

H $-3.9580 \quad 4.8677-4.6326$

H $-5.7253 \quad 5.9454 \quad-3.7682$

H $-6.0035 \quad 4.1792-1.2754$

H $-6.4180 \quad 5.8980-1.3064$ 
$\begin{array}{llll}\mathrm{H} & -2.5972 & 4.8908 & -0.5845\end{array}$

H $-3.6240 \quad 3.6272-1.1344$

H $7.5829 \quad 1.3879 \quad 1.1579$

H $\quad 8.0448 \quad 2.9805 \quad 1.7717$

H $6.34092 .5094 \quad 1.7194$

$\begin{array}{llll}\text { H } & 6.4174 & 2.4539 & -0.7827\end{array}$

H $\quad 8.1310 \quad 2.8406 \quad-0.7578$

$\begin{array}{lllll}\mathrm{H} & 4.6978 & 4.2352 & -0.1784\end{array}$

H $5.4386 \quad 4.6041 \quad 1.3853$

H $5.2192 \quad 5.8846 \quad 0.1835$

H $7.6183 \quad 5.0432 \quad 0.3132$

H $6.8892 \quad 6.1610 \quad-1.7138$

H $5.7557 \quad 3.5751 \quad-2.6738$

H $4.2487 \quad 4.4756-4.8896$

H $3.5711 \quad 3.7345 \quad-3.4442$

H $2.9950 \quad 6.5379 \quad-4.0805$

H $1.2932 \quad 7.3116-2.3475$

H $-1.1418 \quad 5.6517 \quad-3.1434$

H $-0.5089 \quad 5.7889-1.5058$

H $-2.2610 \quad 7.2978-2.0877$

H $8.1771-6.1125-1.5154$

H $7.8203-7.8092-1.9009$

H $9.4651 \quad-3.9365 \quad-7.0173$

H $7.8172 \quad-3.3821-6.6834$

$\begin{array}{llll}\text { H } & 9.1571 & -2.2371 & -6.5847\end{array}$

H $8.7155-4.6008-4.6569$

H $10.1201-3.5397 \quad-4.6450$

$\begin{array}{llll}\mathrm{H} & 0.5022 & 1.2205 & 4.8063\end{array}$

H $-1.1277 \quad 1.6058 \quad 5.3141$

H $\quad 0.1370 \quad 2.8466 \quad 5.4147$

$\begin{array}{llll}\mathrm{H} & 2.6620 & 1.2197 & 5.5671\end{array}$

$\begin{array}{llll}\mathrm{H} & 3.7480 & 1.2383 & 6.9652\end{array}$

$\begin{array}{llll}\text { H } & 2.6537 & -0.1304 & 6.6994\end{array}$

H $\quad 0.0940 \quad 0.2454 \quad 7.0596$

$\begin{array}{llll}\mathrm{H} & -0.4697 & 1.7887 & 7.6722\end{array}$

H $1.8138 \quad 2.7475 \quad 7.3537$

H $1.1236 \quad 1.8864 \quad 9.5710$

H $2.8400 \quad 1.5381 \quad 9.3110$

$\begin{array}{llll}\text { H } & 1.5530 & -2.9597 & 10.0844\end{array}$

H $-3.7212-3.1636 \quad 10.7241$

H $-2.2012-2.2705 \quad 10.5920$

H $-3.7384-1.400810 .6775$

$\begin{array}{llll}\mathrm{H} & 2.7085 & -2.7380 & -3.5300\end{array}$

H $3.4448-3.2036-1.9793$

H $3.7060-4.2006-3.4019$

H $2.0306-5.1651-1.7923$

H $\quad 0.3030 \quad-5.7542 \quad-3.3845$

H $-0.5773-3.2633-3.6345$

H $-0.8822-3.4228-6.5586$

H $-2.6082-1.4302-4.2530$

H $-3.2243-2.6740-6.1757$

$\begin{array}{llll}\text { H } & -2.8099 & -4.0861 & -5.1769\end{array}$

$\begin{array}{lllll}\text { H } & 0.4868 & -1.8472 & -7.3785\end{array}$

H $\quad 0.2653 \quad 0.2125 \quad-8.6300$

$\begin{array}{llll}\mathrm{H} & -0.7503 & 0.8583 & -7.3105\end{array}$

H $7.3451-0.6822-3.4920$

H $-7.15884 .9676 \quad-3.3952$

H $-3.7605 \quad 1.9541 \quad-4.2469$

H $7.9524 \quad 4.8064-2.1338$

H $5.3401 \quad 1.6011 \quad 4.4634$
Н $\quad 0.9129-2.6588-2.0445$

H $1.5634-0.1835-2.1141$

H $0.5095-0.8146 \quad-1.1665$

H $-1.9978 \quad 11.8970-1.8319$

H $-2.19218 .4064 \quad 7.8750$

H $1.9057 \quad-5.8467 \quad-4.1334$

C $-0.3604 \quad-0.1379 \quad 1.2581$

$\begin{array}{llll}\text { O } & -0.2535 & -1.1337 & 0.4629\end{array}$

$\begin{array}{lllll}\text { O } & -0.5695 & -0.1418 & 2.4798\end{array}$

H $\quad 2.5117 \quad-1.7970 \quad 9.1511$

H $\quad 1.6363 \quad 0.2552 \quad 9.1310$

H $1.0113 \quad 0.7535 \quad-7.1045$

$\begin{array}{llll}\mathrm{H} & -8.0325 & -2.1775 & -5.8871\end{array}$

H $\quad 0.6645 \quad 7.1543 \quad-3.9819$

C $-3.4603-5.7659-0.7230$

H $-8.4684 \quad-5.9483 \quad-2.3659$

H $-9.7486-4.7914 \quad-2.2971$

H $-7.5220-2.1833-1.6527$

H $-9.0152-2.8747-1.0957$

H $-5.9347-3.6464-1.8598$

H $-6.5644-6.0888-3.4015$

H $-6.0896-6.2381-1.7005$

H $-4.1986-6.5584-3.3058$

H $-4.4728-4.9523 \quad-3.9677$

H $-3.1968-6.8091 \quad-0.9399$

H $-4.4096-5.7637 \quad-0.1832$

H $-2.5472-4.9023-2.4783$

H $-3.7902-3.9252-1.7564$

H $-1.9673-1.7175 \quad-0.0135$

H $-3.2345-1.7946 \quad 0.8603$

H -11.6996 -5.26312 .9275$

H -11.7085 $-3.7933 \quad 1.9300$

H -11.6894 $-5.3781 \quad 1.1589$

H $-9.3600 \quad-5.7855 \quad 2.1435$

H $-9.4195 \quad-4.2003 \quad 2.8811$

H -11.9297 $-3.9512-2.0838$

H $-10.9790-4.0671-0.8546$

H $-1.5431-4.9905-0.1393$

H $\quad 2.2964 \quad 0.8640 \quad 0.1938$

\section{$\mathrm{TS}$ for $\mathrm{IP} \rightarrow \mathrm{YIH}^{+}$}

$\begin{array}{llll}\mathrm{N} & -2.7884 & -5.0264 & 0.2814\end{array}$

C $-3.7896-4.8044-1.9541$

C $-4.7862-5.3562-2.9818$

$\begin{array}{llll}C & -6.2580 & -5.3301 & -2.5596\end{array}$

$\mathrm{N}-6.7181 \quad-3.9600-2.3576$

C $-8.0226-3.6233-2.2321$

$\mathrm{N}-8.9786 \quad-4.5103 \quad-2.5224$

N $-8.3736 r-2.3569-1.9447$

C - $11.5904-4.0423 \quad 1.9630$

C $-10.0631-4.1352 \quad 1.9897$

$\begin{array}{llll}\text { C } & -9.3531 & -3.6095 & 0.7372\end{array}$

$\begin{array}{llll}\text { O } & -8.2247 & -4.0406 & 0.4535\end{array}$

$\begin{array}{llll}\text { O } & -9.9960 & -2.7058 & 0.0502\end{array}$

O $-11.5551 \quad-3.8925-1.7079$

$\begin{array}{llll}\text { O } & -3.0258 & -1.5056 & -0.0729\end{array}$

C $7.4218-7.6189-0.8072$

C $6.0052 \quad-7.3443-0.3147$

$\begin{array}{llll}\text { O } & 5.0067 & -7.7378 & -0.9103\end{array}$ $\begin{array}{llll}\text { N } & 5.8874 & -6.6305 & 0.8528\end{array}$

C $4.5894 \quad-6.4829 \quad 1.4921$

C $3.8183 \quad-5.2306 \quad 1.0440$

$\begin{array}{llll}\text { O } & 4.3889 & -4.2170 & 0.6250\end{array}$

$\begin{array}{llll}\text { C } & 4.9634 & -6.3393 & 2.9857\end{array}$

$\begin{array}{llll}\text { C } & 6.2970 & -5.5782 & 2.9331\end{array}$

C $6.9934-6.1424 \quad 1.6829$

$\begin{array}{llll}\text { N } & 2.4878 & -5.2937 & 1.2529\end{array}$

$\begin{array}{llll}\text { C } & 1.6082 & -4.1419 & 1.2183\end{array}$

$\begin{array}{llll}\text { C } & 0.2639 & -4.5765 & 1.8010\end{array}$

$\begin{array}{llll}\text { O } & 0.1363 & -5.6710 & 2.3500\end{array}$

N $-0.7507 \quad-3.6858 \quad 1.6543$

$\begin{array}{llll}\text { C } & -2.0171 & -3.9037 & 2.3390\end{array}$

C $-3.1359-4.4896 \quad 1.4670$

O $-4.2973-4.4832 \quad 1.8961$

$\begin{array}{llll}\text { C } & -2.5279 & -2.6384 & 3.0918\end{array}$

$\begin{array}{llll}\text { O } & -3.6496 & -1.9917 & 2.4842\end{array}$

C $8.7532-4.0176-6.1658$

C $8.6360-4.3113-4.6680$

C $7.8752-3.2450 \quad-3.8677$

O $7.9031-2.0531-4.3499$

O $7.3069-3.5806-2.8054$

$\begin{array}{llll}\text { C } & 0.9037 & -5.3398 & -3.5822\end{array}$

C 0.5178 -4.4418 $\quad-4.7617$

$\begin{array}{lllll}\text { O } & 0.9699 & -4.5911 & -5.8938\end{array}$

C $1.4610-4.6242-2.3449$

$\begin{array}{llll}\text { C } & 2.7622 & -3.8777 & -2.6203\end{array}$

$\begin{array}{llll}\text { O } & 0.4501 & -3.7642 & -1.7879\end{array}$

N $-0.4420 \quad-3.4895 \quad-4.5043$

C $-1.2014-2.9163-5.6006$

C $-0.8578-1.4520-5.9503$

$\begin{array}{llll}\text { O } & -1.4325 & -0.5079 & -5.3785\end{array}$

$\begin{array}{llll}\text { C } & -2.7030 & -2.9908 & -5.2988\end{array}$

O $-3.0274-2.2634 \quad-4.1191$

$\begin{array}{llll}\mathrm{N} & 0.0007 & -1.2667 & -6.9704\end{array}$

$\begin{array}{llll}\text { C } & 0.2878 & 0.0517 & -7.5383\end{array}$

C $-7.8275-1.0053-5.2481$

C $-6.4103-1.1729-4.6927$

N $-6.0510-0.0764 \quad-3.7845$

$\begin{array}{llll}\mathrm{C} & -5.4004 & -0.1964 & -2.6218\end{array}$

N $-4.8803-1.3802 \quad-2.2402$

N $-5.3359 \quad 0.8610-1.7935$

C $-5.6586 \quad 5.3197 \quad-3.4543$

C $-5.1040 \quad 3.9968-3.9716$

$\begin{array}{lllll}\text { O } & -5.6748 & 2.9306 & -3.6939\end{array}$

$\begin{array}{llll}\text { C } & -5.3580 & 5.4335 & -1.9388\end{array}$

$\begin{array}{llll}C & -3.9125 & 5.7792 & -1.5770\end{array}$

$\mathrm{N}-3.1428 \quad 4.7945 \quad-1.0988$

$\begin{array}{lllll}\mathrm{O} & -3.5161 & 6.9618 & -1.6823\end{array}$

$\begin{array}{llll}\mathrm{N} & -3.9417 & 4.0533 & -4.6566\end{array}$

C $-3.0180 \quad 2.9164-4.7784$

C $-1.7170 \quad 3.2395-4.0233$

C $-1.0525 \quad 2.0263 \quad-3.3828$

C $-1.9690 \quad 1.3754-2.3638$

$\mathrm{N}-1.9003 \quad 0.0370-2.2800$

$\begin{array}{lllll}\mathrm{O} & -2.7824 & 2.0248 & -1.6717\end{array}$

$\begin{array}{llll}\text { O } & 0.7745 & -1.0768 & -2.0444\end{array}$

$\begin{array}{llll}\text { C } & -2.1507 & 8.1362 & 6.8219\end{array}$

$\begin{array}{llll}\text { C } & -1.4175 & 6.7910 & 6.8021\end{array}$

$\mathrm{N}-1.9065 \quad 5.8657 \quad 5.7756$ 
$\begin{array}{llll}C & -1.4765 & 5.8082 & 4.5017\end{array}$

$\begin{array}{llll}\mathrm{N} & -0.6064 & 6.6944 & 4.0189\end{array}$

$\mathrm{N}-1.9412 \quad 4.8459 \quad 3.6955$

C $-0.3529 \quad 11.9790 \quad-1.3850$

C $0.1554 \quad 10.5817 \quad-1.0533$

$\begin{array}{llll}\mathrm{N} & -0.9084 & 9.7840 & -0.4417\end{array}$

$\begin{array}{llll}\mathrm{C} & -0.6979 & 8.6189 & 0.1981\end{array}$

$\begin{array}{llll}\mathrm{N} & -1.7378 & 7.8285 & 0.4997\end{array}$

$\begin{array}{lllll}\mathrm{N} & 0.5215 & 8.2985 & 0.6361\end{array}$

$\begin{array}{llll}\text { C } & 1.2657 & 6.4657 & -3.1539\end{array}$

$\begin{array}{llll}\text { C } & 2.0939 & 5.1917 & -3.2540\end{array}$

$\begin{array}{lllll}\text { O } & 1.8205 & 4.1640 & -2.6299\end{array}$

$\begin{array}{lllll}C & -0.0986 & 6.2483 & -2.5184\end{array}$

$\begin{array}{lllll}\mathrm{O} & -0.7892 & 7.4925 & -2.4915\end{array}$

$\begin{array}{llll}\text { N } & 3.1519 & 5.3009 & -4.1086\end{array}$

$\begin{array}{llll}\text { C } & 4.2919 & 4.4153 & -4.0059\end{array}$

$\begin{array}{llll}C & 5.3308 & 5.0227 & -3.0480\end{array}$

$\begin{array}{lllll}\text { O } & 5.2437 & 6.1956 & -2.6898\end{array}$

$\begin{array}{lllll}\mathrm{N} & 6.3331 & 4.1794 & -2.7019\end{array}$

C $7.3478 \quad 4.5411 \quad-1.7123$

$\begin{array}{llll}\text { C } & 7.1518 & 3.7846 & -0.3827\end{array}$

$\begin{array}{llll}\text { C } & 7.4578 & 2.2843 & -0.5496\end{array}$

C $5.7471 \quad 4.0306 \quad 0.1875$

$\begin{array}{llll}\text { C } & 7.4101 & 1.4789 & 0.7499\end{array}$

C $1.8233 \quad 1.3809 \quad 8.9607$

C $\quad 1.5407 \quad 1.8218 \quad 7.5155$

C $0.0774 \quad 1.5332 \quad 7.1362$

$\begin{array}{llll}\text { C } & 2.5320 & 1.1595 & 6.5510\end{array}$

$\begin{array}{llll}\text { C } & -0.3479 & 2.0546 & 5.7584\end{array}$

$\begin{array}{llll}\text { C } & 1.4101 & -2.3987 & 9.1934\end{array}$

C $1.4919 \quad-3.4530 \quad 8.0766$

$\begin{array}{llll}\text { C } & 1.6255 & -2.8512 & 6.6946\end{array}$

$\begin{array}{llll}\text { C } & 0.5544 & -2.1475 & 6.1261\end{array}$

$\begin{array}{llll}\text { C } & 2.8050 & -2.9715 & 5.9510\end{array}$

C $0.6556-1.5745 \quad 4.8606$

C $2.9110 \quad-2.4088 \quad 4.6753$

C $1.8381-1.7042 \quad 4.1286$

$\begin{array}{llll}\text { C } & -3.4944 & -1.8556 & 10.2783\end{array}$

C $-3.6254-1.9456 \quad 8.7555$

$\begin{array}{llll}\text { C } & -2.8873 & -0.8438 & 7.9744\end{array}$

$\begin{array}{llll}\text { C } & -2.9776 & -1.1047 & 6.4664\end{array}$

$\begin{array}{llll}\text { C } & -3.4099 & 0.5584 & 8.3094\end{array}$

$\begin{array}{llll}\text { C } & -0.0260 & 2.5043 & 1.3755\end{array}$

$\begin{array}{llll}C & 0.1557 & 4.9959 & 1.2477\end{array}$

$\begin{array}{lllll}\text { O } & 0.3005 & 1.3301 & -0.6736\end{array}$

$\begin{array}{llll}\text { O } & -1.0956 & 5.1841 & 1.0409\end{array}$

$\begin{array}{llll}\text { O } & 0.8819 & 5.8395 & 1.8538\end{array}$

$\begin{array}{llll}\text { C } & 6.6781 & 1.0140 & -4.6743\end{array}$

$\begin{array}{llll}C & 5.6526 & -1.7417 & 2.0427\end{array}$

C $3.7897-1.2036-0.2625$

C $5.6170-1.2539-1.9680$

$\begin{array}{llll}\text { C } & 4.5624 & -0.5989 & -1.3974\end{array}$

$\begin{array}{llll}C & 4.1338 & 0.6506 & -1.9974\end{array}$

N $3.0439 \quad 1.2566-1.5515$

$\begin{array}{llll}\mathrm{N} & 4.8621 & 1.1784 & -3.0430\end{array}$

$\begin{array}{llll}\text { C } & 5.8719 & 0.4769 & -3.5274\end{array}$

N 6.2598 -0.7119 $\quad-3.0325$

$\begin{array}{llll}\mathrm{S} & 3.3668 & 1.5429 & 2.4899\end{array}$

$\begin{array}{llll}\text { C } & 3.0920 & 0.7423 & 1.0058\end{array}$

N $3.8517 \quad-0.3557 \quad 0.9612$ $\begin{array}{llll}\text { C } & 4.6979 & -0.5963 & 2.0516\end{array}$

$\begin{array}{llll}C & 4.5455 & 0.3569 & 3.0151\end{array}$

$\begin{array}{llll}C & 5.2115 & 0.4378 & 4.3516\end{array}$

$\begin{array}{llll}\text { C } & 0.0484 & 1.2677 & 0.5140\end{array}$

$\begin{array}{llll}C & 0.7466 & 3.6874 & 0.7802\end{array}$

H $2.8208 \quad 2.1318 \quad-2.0267$

H $2.7810 \quad 1.1119 \quad-0.2012$

H $-1.0965 \quad 2.7484 \quad 1.4142$

H $\quad 0.2713 \quad 2.2638 \quad 2.3980$

H $7.4697 \quad 1.6729-4.2982$

H $\quad \begin{array}{llll}6.0385 & 1.5957 & -5.3399\end{array}$

H $7.1594 \quad 0.1960 \quad-5.2148$

$\begin{array}{llll}\text { H } & 5.1431 & -2.6950 & 1.8837\end{array}$

H $6.2008 \quad-1.7787 \quad 2.9846$

H $6.3777 \quad-1.6322 \quad 1.2291$

H $4.6645 \quad-0.1548 \quad 5.0914$

H $0.6794 \quad 3.6441 \quad-0.3131$

H $5.9978 \quad-2.2355-1.6854$

H $\quad 2.7305-1.2812 \quad-0.5170$

H $\quad 6.2345 \quad 0.0583 \quad 4.2926$

H $1.8047 \quad 3.6524 \quad 1.0467$

H $4.1447-2.2020-0.0110$

H $-0.1710 \quad-1.0249 \quad 4.4289$

H $1.9069-1.2563 \quad 3.1432$

$\begin{array}{llll}\mathrm{H} & -0.3717 & -2.0533 & 6.6843\end{array}$

H $3.6447 \quad-3.5198 \quad 6.3701$

H $3.8303 \quad-2.5286 \quad 4.1105$

H $\quad 0.5910 \quad-4.0781 \quad 8.1096$

H $\quad 2.3385 \quad-4.1210 \quad 8.2677$

H $0.5604 \quad-1.7268 \quad 9.0372$

H $-3.2441 \quad-2.9190 \quad 8.4209$

H $-4.6876 \quad-1.9248 \quad 8.4747$

$\begin{array}{llll}\mathrm{H} & -4.4821 & 0.6318 & 8.0901\end{array}$

$\begin{array}{llll}\mathrm{H} & -3.2660 & 0.8091 & 9.3642\end{array}$

H $-2.8938 \quad 1.3189 \quad 7.7135$

H $-1.8262-0.8843 \quad 8.2657$

H $-4.0148 \quad-1.0416 \quad 6.1181$

H $-2.3897-0.3805 \quad 5.8925$

$\begin{array}{llll}\mathrm{H} & -2.6094 & -2.1042 & 6.2161\end{array}$

H $-2.3044 \quad 3.9906 \quad 4.0873$

H $-1.7434 \quad 4.89492 .6856$

$\mathrm{H}-0.3597 \quad 7.5058 \quad 4.5605$

H $-0.0482 \quad 6.4619 \quad 3.1717$

$\mathrm{H}-2.7211 \quad 5.3125 \quad 5.9987$

$\begin{array}{llll}\mathrm{H} & -0.3417 & 6.9225 & 6.6501\end{array}$

H $-1.5279 \quad 6.2818 \quad 7.7626$

H $-2.0486 \quad 8.6663 \quad 5.8699$

H $-3.2179 \quad 7.9958 \quad 7.0141$

H $-1.5100 \quad 6.8815 \quad 0.8443$

H $-2.5274 \quad 7.8299-0.1441$

H $-1.8239 \quad 9.8857 \quad-0.8581$

H $\quad 1.3133 \quad 8.8674 \quad 0.3919$

H $\quad 0.6915 \quad 7.3889 \quad 1.0930$

H $\quad 0.5329 \quad 10.0673 \quad-1.9457$

H $\quad 0.9764 \quad 10.6750 \quad-0.3354$

H $\quad 0.4484 \quad 12.5701 \quad-1.8344$

H $-0.7014 \quad 12.4920-0.4850$

$\begin{array}{lllll}\text { H } & 8.0742 & -7.9934 & -0.0136\end{array}$

H $7.6698-6.9643 \quad 1.9494$

H $7.5771 \quad-5.3818 \quad 1.1556$
H $6.8991 \quad-5.7116 \quad 3.8351$

H $6.1122 \quad-4.5096 \quad 2.8041$

H $5.1048 \quad-7.3364 \quad 3.4140$

H $4.1934 \quad-5.8259 \quad 3.5677$

H $3.9824 \quad-7.3678 \quad 1.2846$

H $2.0645-6.1106 \quad 1.6837$

$\begin{array}{llll}\text { H } & 1.4521 & -3.7661 & 0.2041\end{array}$

H $2.0122 \quad-3.3247 \quad 1.8285$

H $-0.5508 \quad-2.7655 \quad 1.2460$

H $-1.8183 \quad-4.6870 \quad 3.0785$

H $-1.7300 \quad-1.8979 \quad 3.1283$

H $-2.7944 \quad-2.9242 \quad 4.1134$

H $-4.3167-2.7047 \quad 2.3896$

H $-4.4431-1.9209-2.9932$

H $-4.3072-1.3824-1.3859$

H $-4.4605 \quad 0.9774-1.2823$

H $-5.6593 \quad 1.7370-2.1981$

H $-6.2328 \quad 0.8779 \quad-4.0823$

H $-6.3444-2.1066-4.1316$

H $-5.6738-1.2221 \quad-5.5038$

H $-7.9386-0.0561 \quad-5.7827$

H $-8.5565-1.0503-4.4357$

H $-1.0443-0.4175-2.5975$

H $-2.3361 \quad-0.3706 \quad-1.4562$

H $-0.7667 \quad 1.2841 \quad-4.1314$

H $-0.1474 \quad 2.3451 \quad-2.8551$

H $-1.00413 .7404-4.6868$

H $-1.96793 .9388-3.2243$

H $-2.8334 \quad 2.6870-5.8319$

H $-3.5460 \quad 4.9716-4.8061$

H $-5.2431 \quad 6.1827 \quad-3.9835$

H $-5.66694 .5076-1.4466$

H $-5.9594 \quad 6.2488-1.5285$

H $-2.2352 \quad 5.0029-0.6872$

H $-3.3407 \quad 3.8017-1.2190$

$\begin{array}{llll}\text { H } & 7.6942 & 0.4376 & 0.5699\end{array}$

$\begin{array}{llll}\mathrm{H} & 8.0993 & 1.8883 & 1.4971\end{array}$

$\begin{array}{llll}\mathrm{H} & 6.4081 & 1.4787 & 1.1865\end{array}$

H $6.7403 \quad 1.8460 \quad-1.2534$

H $8.4495 \quad 2.1731-1.0063$

H $4.9864 \quad 3.5137-0.4081$

H $\begin{array}{llll}5.6635 & 3.6671 & 1.2151\end{array}$

H $\begin{array}{llll}5.5019 & 5.0967 & 0.1831\end{array}$

$\begin{array}{llll}\mathrm{H} & 7.8907 & 4.2012 & 0.3171\end{array}$

H $7.2567 \quad 5.6180-1.5636$

H $\quad 6.1728 \quad 3.1973-2.8940$

H $4.7497 \quad 4.2874-4.9931$

H $3.9732 \quad 3.4285 \quad-3.6690$

H $3.4140 \quad 6.2552 \quad-4.3244$

H $\quad 1.8390 \quad 7.1865 \quad-2.5557$

H $-0.6506 \quad 5.5063 \quad-3.1035$

H $\quad 0.0254 \quad 5.8318-1.5100$

H $-1.7223 \quad 7.3020-2.2762$

H $7.8572 \quad-6.6939-1.2039$

H $7.3585-8.3507-1.6114$

H $9.3536-4.7792 \quad-6.6748$

H $7.7658-3.9950-6.6388$

H $\quad 9.2134-3.0397 \quad-6.3234$

H $\quad 8.1507 \quad-5.2744 \quad-4.4811$

H $\quad 9.6390 \quad-4.3850 \quad-4.2247$ 
$\begin{array}{llll}\mathrm{H} & 0.1483 & 1.5199 & 4.9439\end{array}$

H -1.4264 $1.9184 \quad 5.6109$

H $-0.1200 \quad 3.1237 \quad 5.6529$

H $2.3759 \quad 1.4833 \quad 5.5176$

H $3.5614 \quad 1.4085 \quad 6.8290$

H $2.4285 \quad 0.0701 \quad 6.5722$

$\begin{array}{llll}\mathrm{H} & -0.0912 & 0.4507 & 7.1748\end{array}$

$\begin{array}{llll}\mathrm{H} & -0.5726 & 1.9717 & 7.9044\end{array}$

H $1.6883 \quad 2.9121 \quad 7.4577$

H $1.1557 \quad 1.8832 \quad 9.6692$

H $2.8542 \quad 1.6096 \quad 9.2501$

H $1.2880 \quad-2.8734 \quad 10.1717$

H $-3.9761 \quad-2.7095 \quad 10.7637$

H $-2.4413-1.8509 \quad 10.5831$

H -3.9589 -0.9482 10.6762

$\begin{array}{llll}\text { H } & 2.5975 & -3.0362 & -3.3014\end{array}$

H $3.1875-3.5045-1.6875$

H $3.5032-4.5443-3.0686$

H $1.6600 \quad-5.3997-1.5952$

H $0.0071 \quad-5.8956-3.2822$

H $-0.7620 \quad-3.3700 \quad-3.5502$

H $-0.9823-3.5362-6.4750$

H $-2.6278-1.3752-4.2512$

H $-3.2708-2.6186-6.1637$

H $-2.9802-4.0332-5.1263$

$\begin{array}{lllll}\text { H } & 0.5103 & -2.0773 & -7.2999\end{array}$

$\begin{array}{llll}\text { H } & 0.4232 & -0.0426 & -8.6173\end{array}$

$\begin{array}{llll}\mathrm{H} & -0.5614 & 0.7012 & -7.3257\end{array}$

H $7.0048 \quad-1.2760-3.5329$

$\begin{array}{llll}\mathrm{H} & -6.7406 & 5.3099 & -3.5987\end{array}$

$\begin{array}{llll}\mathrm{H} & -3.5334 & 2.0619 & -4.3404\end{array}$

$\begin{array}{llll}\text { H } & 8.3427 & 4.3318 & -2.1220\end{array}$

H $\quad 5.2579 \quad 1.4677 \quad 4.7117$

H $0.7136 \quad-2.8333-1.9530$

H $1.4017 \quad-0.3397 \quad-2.1399$

$\begin{array}{llll}\text { H } & 0.4043 & -0.9432 & -1.1320\end{array}$

H $-1.177411 .9396-2.1048$

H $-1.7429 \quad 8.7715 \quad 7.6129$

H $1.6363-6.0554-3.9605$

$\begin{array}{llll}\text { C } & -0.3036 & -0.0866 & 1.1927\end{array}$

O $-0.2975-1.1097 \quad 0.4189$

$\begin{array}{llll}\text { O } & -0.5744 & -0.0460 & 2.3993\end{array}$

H $\quad 2.3202 \quad-1.7927 \quad 9.2202$

H $1.6763 \quad 0.3045 \quad 9.0698$

H $1.1887 \quad 0.4939 \quad-7.0999$

$\begin{array}{llll}\mathrm{H} & -8.0398 & -1.8143 & -5.9520\end{array}$

H $1.1407 \quad 6.9125 \quad-4.1460$

C $-3.7846-5.5725-0.6316$

H $-8.7677 \quad-5.4845 \quad-2.3702$

H $-9.9658-4.2413-2.3441$

H $-7.5776-1.7834-1.6923$

H $-9.1253-2.3634-1.1480$

H $-6.0951-3.3566-1.8363$

H $-6.8581-5.7726-3.3604$

H $-6.4197-5.9230-1.6495$

H $-4.5273-6.3969-3.2102$

H $-4.6904-4.7943-3.9173$

H $-3.5822-6.6368-0.8073$

H $-4.7423-5.4923-0.1130$

H $-2.7856-4.8312-2.3953$
H $-3.9737-3.7504-1.7307$

H $-2.0538-1.5804 \quad 0.0097$

$\begin{array}{llll}\mathrm{H} & -3.3350 & -1.5418 & 0.8685\end{array}$

$\mathrm{H}-12.0241 \quad-4.4415 \quad 2.8853$

H -11.9096 $-3.0034 \quad 1.8491$

H - $12.0052-4.6057 \quad 1.1208$

$\begin{array}{llll}\mathrm{H} & -9.7241 & -5.1641 & 2.1440\end{array}$

H $-9.6683 \quad-3.5588 \quad 2.8378$

H -12.0799 $-3.2364 \quad-2.1823$

H -11.1652 $-3.4044 \quad-0.9329$

H $-1.8325-4.9055-0.0295$

\section{YI}

N $-3.2724 \quad-4.7248 \quad 0.4669$

$\begin{array}{llll}\text { C } & -4.3416 & -4.4917 & -1.7335\end{array}$

C $-5.4333-4.9766-2.6964$

$\begin{array}{llll}\text { C } & -6.8756 & -4.7909 & -2.2152\end{array}$

N $-7.1864-3.3776-2.0252$

$\begin{array}{llll}\text { C } & -8.4443 & -2.9071 & -1.8531\end{array}$

N $-9.4972-3.6930-2.0952$

N $-8.6500 \quad-1.6096-1.5661$

C $-11.8459-2.8020 \quad 2.5434$

C $-10.3522 \quad-3.1260 \quad 2.4721$

$\begin{array}{llll}\text { C } & -9.6479 & -2.7092 & 1.1755\end{array}$

$\begin{array}{lllll}\text { O } & -8.5851 & -3.2644 & 0.8572\end{array}$

$\begin{array}{llll}\text { O }-10.2224 & -1.7594 & 0.4898\end{array}$

O $-11.9611-2.8121 \quad-1.1812$

$\begin{array}{llll}\text { O } & -3.2709 & -1.1987 & 0.1328\end{array}$

C $6.5356-8.4055-1.0133$

C $5.2425 \quad-7.9182 \quad-0.3666$

$\begin{array}{lllll}\text { O } & 4.1542 & -8.4379 & -0.5956\end{array}$

N $5.3551 \quad-6.8675 \quad 0.5123$

C $4.2417 \quad-6.5222 \quad 1.3859$

C $3.4286-5.3045 \quad 0.9229$

$\begin{array}{llll}\text { O } & 3.9521 & -4.3359 & 0.3524\end{array}$

C $4.9574 \quad-6.1664 \quad 2.7104$

$\begin{array}{llll}\text { C } & 6.2588 & -5.4996 & 2.2351\end{array}$

C $6.6135 \quad-6.23390 .9262$

N $2.1346 \quad-5.3185 \quad 1.2947$

C $1.2700-4.1569 \quad 1.2382$

C $-0.0586-4.5260 \quad 1.8952$

$\begin{array}{llll}\mathrm{O} & -0.1904 & -5.5833 & 2.5102\end{array}$

$\begin{array}{llll}\mathrm{N} & -1.0448 & -3.6056 & 1.7398\end{array}$

$\begin{array}{llll}\text { C } & -2.2837 & -3.7128 & 2.4926\end{array}$

$\begin{array}{llll}\text { C } & -3.4934 & -4.2087 & 1.6913\end{array}$

$\begin{array}{llll}\mathrm{O} & -4.6188 & -4.1363 & 2.2038\end{array}$

$\begin{array}{llll}\text { C } & -2.6477 & -2.3930 & 3.2453\end{array}$

$\begin{array}{llll}\text { O } & -3.7758 & -1.6927 & 2.7142\end{array}$

C $7.9848-5.0408-6.4937$

C $7.8681-5.5348-5.0468$

C $7.1780-4.6013-4.0617$

O $7.0984-3.3420-4.4945$

$\begin{array}{lllll}\text { O } & 6.7621 & -4.9680 & -2.9734\end{array}$

C $0.1696 \quad-5.4900 \quad-3.5341$

C $-0.1650-4.5366-4.6896$

$\begin{array}{llll}\text { O } & 0.2903 & -4.6772 & -5.8227\end{array}$

C $0.7356-4.8458-2.2650$

$\begin{array}{llll}\text { C } & 2.0377 & -4.0923 & -2.5288\end{array}$

O $-0.2666-4.0196-1.6518$
$\mathrm{N}-1.0756-3.5431 \quad-4.4190$

C $-1.7831-2.8883-5.5049$

C $-1.2896-1.4697-5.8790$

O $-1.7354-0.4663-5.2941$

C $-3.2779-2.7924-5.1696$

$\begin{array}{lllll}\text { O } & -3.5001 & -2.0240 & -3.9920\end{array}$

$\mathrm{N}-0.4590 \quad-1.3874-6.9376$

C $-0.0622-0.1198 \quad-7.5598$

C $-8.1273-0.2813-4.8863$

$\begin{array}{llll}\text { C } & -6.7050 & -0.5930 & -4.4127\end{array}$

$\mathrm{N}-6.1803 \quad 0.4611 \quad-3.5351$

$\begin{array}{llll}\mathrm{C} & -5.5410 & 0.2775 & -2.3746\end{array}$

$\begin{array}{llll}\mathrm{N} & -5.1722 & -0.9594 & -1.9800\end{array}$

$\begin{array}{llll}\mathrm{N} & -5.3414 & 1.3317 & -1.5665\end{array}$

C $-5.2254 \quad 5.8038-3.3122$

$\begin{array}{llll}\text { C } & -4.8285 & 4.4189 & -3.8092\end{array}$

$\begin{array}{lllll}\text { O } & -5.4862 & 3.4212 & -3.4746\end{array}$

C $-4.8566 \quad 5.9421 \quad-1.8159$

C $-3.3694 \quad 6.1510-1.5291$

$\mathrm{N}-2.6835 \quad 5.1040 \quad-1.0632$

$\begin{array}{lllll}\mathrm{O} & -2.8604 & 7.2844 & -1.6919\end{array}$

N $-3.7061 \quad 4.3453 \quad-4.5562$

$\begin{array}{llll}\text { C } & -2.9164 & 3.1167 & -4.7083\end{array}$

C $-1.5659 \quad 3.2833-3.9875$

C $-1.0679 \quad 2.0176-3.3010$

C $-2.0374 \quad 1.5496-2.2318$

$\mathrm{N}-2.2205 \quad 0.2230 \quad-2.1436$

$\begin{array}{llll}\mathrm{O} & -2.6728 & 2.3336 & -1.4951\end{array}$

$\begin{array}{lllll}\text { O } & 0.0717 & -1.4061 & -2.1733\end{array}$

$\begin{array}{llll}\text { C } & -0.9639 & 8.3785 & 6.7401\end{array}$

$\begin{array}{llll}C & -0.3297 & 6.9881 & 6.6166\end{array}$

N $-0.9794 \quad 6.1254 \quad 5.6243$

$\begin{array}{llll}\mathrm{C} & -0.6069 & 5.9599 & 4.3415\end{array}$

$\begin{array}{llll}\mathrm{N} & 0.3391 & 6.7142 & 3.7829\end{array}$

N $-1.2142 \quad 5.0248 \quad 3.5996$

C $0.8405 \quad 11.8939$-1.6109

C $1.2165 \quad 10.4469 \quad-1.3131$

$\begin{array}{lllll}\mathrm{N} & 0.1145 & 9.7627 & -0.6358\end{array}$

$\begin{array}{llll}C & 0.2344 & 8.5860 & 0.0070\end{array}$

$\begin{array}{llll}\mathrm{N} & -0.8667 & 7.9206 & 0.3813\end{array}$

$\begin{array}{llll}\mathrm{N} & 1.4349 & 8.1331 & 0.3770\end{array}$

$\begin{array}{llll}\text { C } & 1.7873 & 6.2161 & -3.3462\end{array}$

C $2.5022 \quad 4.8766 \quad-3.4684$

$\begin{array}{lllll}\text { O } & 2.1627 & 3.8765 & -2.8255\end{array}$

$\begin{array}{lllll}C & 0.3783 & 6.1171 & -2.7837\end{array}$

$\begin{array}{llll}\text { O } & -0.1419 & 7.4341 & -2.6546\end{array}$

$\begin{array}{lllll}\mathrm{N} & 3.5386 & 4.8859 & -4.3484\end{array}$

C $4.5684 \quad 3.8639-4.3350$

C $5.7607 \quad 4.3568-3.4990$

$\begin{array}{lllll}\text { O } & 5.9634 & 5.5659 & -3.3849\end{array}$

$\begin{array}{llll}\mathrm{N} & 6.5378 & 3.3790 & -2.9849\end{array}$

C $7.69493 .6804-2.1443$

C $7.4723 \quad 3.3349-0.6595$

$\begin{array}{llll}\text { C } & 7.5283 & 1.8162 & -0.4145\end{array}$

$\begin{array}{llll}\text { C } & 6.1748 & 3.9649 & -0.1331\end{array}$

C $7.5105 \quad 1.4318 \quad 1.0660$

C $\quad 2.3756 \quad 1.2720 \quad 8.8250$

C $2.1804 \quad 1.5983 \quad 7.3391$

$\begin{array}{llll}\text { C } & 0.6939 & 1.4964 & 6.9522\end{array}$

C 3.06820 .68996 .4846 
$\begin{array}{llll}C & 0.3585 & 1.9806 & 5.5368\end{array}$

C $1.5796 \quad-2.4392 \quad 9.1477$

$\begin{array}{llll}\text { C } & 1.5215 & -3.5153 & 8.0479\end{array}$

C $1.6803 \quad-2.9579 \quad 6.6490$

C $0.6362 \quad-2.2334 \quad 6.0565$

C $2.8615 \quad-3.1339 \quad 5.9178$

$\begin{array}{llll}\text { C } & 0.7661 & -1.6912 & 4.7796\end{array}$

$\begin{array}{llll}\text { C } & 2.9974 & -2.5995 & 4.6331\end{array}$

$\begin{array}{llll}\text { C } & 1.9512 & -1.8733 & 4.0638\end{array}$

C $-3.1846 \quad-1.3658 \quad 10.4422$

$\begin{array}{llll}\text { C } & -3.3832 & -1.4632 & 8.9271\end{array}$

$\begin{array}{llll}\text { C } & -2.5300 & -0.4895 & 8.0946\end{array}$

$\begin{array}{llll}\text { C } & -2.7132 & -0.7631 & 6.5975\end{array}$

$\begin{array}{llll}\text { C } & -2.8402 & 0.9784 & 8.4118\end{array}$

C $\quad 0.1810 \quad 2.4390 \quad 1.0575$

C $\quad 0.7236 \quad 4.8808 \quad 1.0000$

$\begin{array}{lllll}\mathrm{O} & 0.5186 & 1.0584 & -0.8708\end{array}$

$\begin{array}{llll}\text { O } & -0.5063 & 5.2292 & 0.8844\end{array}$

O $1.5882 \quad 5.6275 \quad 1.5487$

$\begin{array}{llll}\text { C } & 6.7459 & 0.0449 & -4.5159\end{array}$

C $5.5838-1.7792 \quad 1.5244$

C $3.2272-1.2190-0.1905$

C 4.9776 -1.8109 -1.8998

$\begin{array}{llll}\text { C } & 4.1410 & -0.8679 & -1.3344\end{array}$

$\begin{array}{llll}\text { C } & 4.1559 & 0.4290 & -1.9353\end{array}$

N $3.3482 \quad 1.4197 \quad-1.5234$

$\begin{array}{llll}\text { N } & 5.0095 & 0.6996 & -2.9538\end{array}$

C $5.7833-0.2913-3.4110$

N $5.7933 \quad-1.5443 \quad-2.9415$

$\begin{array}{llll}\text { S } & 3.3273 & 1.2986 & 2.7790\end{array}$

$\begin{array}{llll}\text { C } & 2.6869 & 0.5937 & 1.3484\end{array}$

$\begin{array}{llll}\text { N } & 3.5047 & -0.4238 & 1.0294\end{array}$

$\begin{array}{llll}\text { C } & 4.6250 & -0.6827 & 1.8506\end{array}$

$\begin{array}{llll}\text { C } & 4.6771 & 0.1813 & 2.8983\end{array}$

C $5.6535 \quad 0.2102 \quad 4.0307$

$\begin{array}{llll}\text { C } & 0.2617 & 1.1245 & 0.3267\end{array}$

$\begin{array}{llll}\text { C } & 1.1127 & 3.5128 & 0.4914\end{array}$

H $3.2063 \quad 2.2526 \quad-2.0881$

H $2.6662 \quad 1.2560 \quad-0.7917$

$\begin{array}{llll}\mathrm{H} & -0.8620 & 2.7630 & 0.9273\end{array}$

$\begin{array}{llll}\mathrm{H} & 0.3311 & 2.2670 & 2.1239\end{array}$

H $7.6656 \quad 0.4630-4.0895$

H $6.3183 \quad 0.7971 \quad-5.1815$

H $7.0164-0.8478-5.0808$

H $5.0662 \quad-2.7159 \quad 1.3049$

$\begin{array}{llll}\text { H } & 6.2608 & -1.9450 & 2.3637\end{array}$

H $6.1884-1.5300 \quad 0.6461$

H $5.2655 \quad-0.3477 \quad 4.8895$

$\begin{array}{llll}\text { H } & 1.0424 & 3.5183 & -0.6029\end{array}$

H $5.0015 \quad-2.8317-1.5222$

H $2.1822-1.0208-0.4316$

H $6.6080 \quad-0.2314 \quad 3.7349$

$\begin{array}{llll}\text { H } & 2.1509 & 3.3073 & 0.7550\end{array}$

H $3.3262-2.2795 \quad 0.0484$

H $-0.0368-1.1201 \quad 4.3295$

H $2.0471 \quad-1.4410 \quad 3.0752$

H $-0.2887-2.0966 \quad 6.6077$

H $3.6803 \quad-3.6970 \quad 6.3583$

H $3.9205 \quad-2.7458 \quad 4.0792$

H $0.5626 \quad-4.0432 \quad 8.1171$
H $\quad 2.3002 \quad-4.2633 \quad 8.2323$

H $\quad 0.7982-1.6869 \quad 9.0006$

$\begin{array}{llll}\mathrm{H} & -3.1527 & -2.4860 & 8.6023\end{array}$

H $-4.4423-1.3007 \quad 8.6825$

H $-3.8993 \quad 1.1963 \quad 8.2264$

H $-2.6237 \quad 1.2284 \quad 9.4543$

$\begin{array}{llll}\mathrm{H} & -2.2445 & 1.6458 & 7.7798\end{array}$

H $-1.4743 \quad-0.6712 \quad 8.3484$

$\begin{array}{llll}\mathrm{H} & -3.7416 & -0.5532 & 6.2811\end{array}$

H $-2.0445-0.1467 \quad 5.9874$

H $-2.5080 \quad-1.8115 \quad 6.3603$

H $-1.6340 \quad 4.2219 \quad 4.0427$

H $-1.0652 \quad 5.0152 \quad 2.5805$

H $\quad 0.6855 \quad 7.5289 \quad 4.2613$

H $\quad 0.8235 \quad 6.3830 \quad 2.9238$

H $-1.8396 \quad 5.6732 \quad 5.8988$

$\begin{array}{lllll}\mathrm{H} & 0.7314 & 7.0532 & 6.3593\end{array}$

$\mathrm{H} \quad-0.3771 \quad 6.4621 \quad 7.5740$

H $-0.9098 \quad 8.9290 \quad 5.7958$

H $-2.0164 \quad 8.3036 \quad 7.0270$

$\begin{array}{llll}\mathrm{H} & -0.7261 & 6.9533 & 0.7199\end{array}$

H $-1.6892 \quad 8.0104 \quad-0.2137$

H $-0.8097 \quad 9.9630 \quad-0.9932$

H $\quad 2.2685 \quad 8.6070 \quad 0.0757$

H $1.5293 \quad 7.2026 \quad 0.8165$

H $1.4802 \quad 9.9037 \quad-2.2290$

H $\quad 2.0871 \quad 10.4455 \quad-0.6497$

H $1.6688 \quad 12.3980-2.1140$

H $\quad 0.6107 \quad 12.4353-0.6896$

H $7.2619 \quad-8.7313-0.2622$

H $7.3839-6.9934 \quad 1.0969$

$\begin{array}{llll}\text { H } & 6.9749 & -5.5494 & 0.1525\end{array}$

H $7.0606 \quad-5.5713 \quad 2.9742$

H $6.0828 \quad-4.4440 \quad 2.0300$

H $5.1695 \quad-7.0932 \quad 3.2523$

H $4.3548 \quad-5.5246 \quad 3.3594$

H $3.5819-7.38851 .4698$

H $1.7389 \quad-6.0972 \quad 1.8132$

H $1.0825 \quad-3.8307 \quad 0.2144$

H $1.7112 \quad-3.3174 \quad 1.7877$

H $-0.8191 \quad-2.7198 \quad 1.2712$

H $-2.1103 \quad-4.4993 \quad 3.2347$

H $-1.8065-1.7032 \quad 3.1829$

H $-2.8374-2.6293 \quad 4.2960$

H $-4.4885-2.36592 .6800$

H $-4.8202-1.5525 \quad-2.7364$

H $-4.5831-1.0205-1.1381$

H $-4.4799 \quad 1.3449-1.0252$

H $-5.5500 \quad 2.2378-1.9806$

H $-6.2608 \quad 1.4277 \quad-3.8376$

H $-6.7042-1.5285-3.8512$

H $-6.0252-0.7181 \quad-5.2636$

H $-8.17290 .6760 \quad-5.4159$

H $-8.8093-0.2554-4.0333$

H $-1.5002-0.3944 \quad-2.5220$

H $-2.6537-0.1048-1.2824$

H $-0.9075 \quad 1.2126-4.0213$

H $-0.1153 \quad 2.2228 \quad-2.8018$

$\begin{array}{lllll}\mathrm{H} & -0.8028 & 3.6440 & -4.6848\end{array}$

H $-1.69314 .0462-3.2183$
H $-2.7880 \quad 2.8799 \quad-5.7685$

H $-3.2256 \quad 5.2155-4.7395$

H $-4.7501 \quad 6.6035-3.8885$

H $-5.2340 \quad 5.0700 \quad-1.2758$

H $-5.3573 \quad 6.8282-1.4175$

H $-1.7391 \quad 5.2164-0.6978$

H $-2.9985 \quad 4.1363-1.1295$

H $\begin{array}{llll}7.6434 & 0.3535 & 1.1932\end{array}$

H $8.3187 \quad 1.9328 \quad 1.6108$

$\begin{array}{llll}\mathrm{H} & 6.5642 & 1.7035 & 1.5393\end{array}$

H $6.6855 \quad 1.3323 \quad-0.9242$

H $8.4404 \quad 1.4190 \quad-0.8789$

H $\begin{array}{llll}5.2985 & 3.4422 & -0.5315\end{array}$

$\begin{array}{llll}\text { H } & 6.1199 & 3.9144 & 0.9574\end{array}$

H $\quad 6.1017 \quad 5.0155 \quad-0.4298$

H $8.3198 \quad 3.7840 \quad-0.1208$

H $7.8806 \quad 4.7494-2.2608$

H $6.1517 \quad 2.4349 \quad-2.9975$

H $4.9155 \quad 3.6879-5.3595$

H $4.15992 .9255 \quad-3.9640$

H $3.9010 \quad 5.8018-4.5873$

H $2.3887 \quad 6.8463-2.6765$

H $-0.2379 \quad 5.5170 \quad-3.4609$

H $0.4032 \quad 5.5925-1.8199$

H $-1.0797 \quad 7.3582 \quad-2.3907$

H $6.9895 \quad-7.6020 \quad-1.6045$

H $6.2856-9.2423 \quad-1.6639$

H $8.4980 \quad-5.7864 \quad-7.1079$

H $6.9980 \quad-4.8609$-6.9297

H $8.5451-4.1046-6.5441$

H $7.3361-6.4896-4.9908$

H $8.8659 \quad-5.7293 \quad-4.6317$

H $0.8101 \quad 1.3516 \quad 4.7656$

$\begin{array}{llll}\mathrm{H} & -0.7248 & 1.9572 & 5.3647\end{array}$

H $\quad 0.7092 \quad 3.0095 \quad 5.3781$

$\begin{array}{llll}\mathrm{H} & 2.9495 & 0.8902 & 5.4195\end{array}$

$\begin{array}{llll}\mathrm{H} & 4.1232 & 0.8332 & 6.7421\end{array}$

H $\quad 2.8210 \quad-0.3629 \quad 6.6468$

H $\quad 0.3742 \quad 0.4529 \quad 7.0584$

$\begin{array}{llll}\mathrm{H} & 0.1064 & 2.0729 & 7.6794\end{array}$

H $2.4955 \quad 2.6409 \quad 7.1732$

H $1.8223 \quad 1.9675 \quad 9.4663$

$\begin{array}{llll}\mathrm{H} & 3.4320 & 1.3234 & 9.1086\end{array}$

H $1.4383 \quad-2.883910 .1373$

H $-3.7606-2.1367 \quad 10.9627$

H $-2.1302-1.5007 \quad 10.7103$

H $-3.5052-0.3961 \quad 10.8353$

H $1.8714 \quad-3.2440 \quad-3.2001$

H $2.4744-3.7329-1.5963$

H $2.7709-4.7570-2.9936$

H $0.9419 \quad-5.6580 \quad-1.5578$

H $-0.7497-6.0248 \quad-3.2675$

H $-1.3997-3.4157 \quad-3.4684$

H $-1.6579-3.5308-6.3816$

H $-3.0211-1.1814 \quad-4.1479$

H $-3.8188-2.3685-6.0278$

H $-3.6644-3.7965-4.9805$

H $-0.0541-2.2549-7.2684$

H $-0.0741-0.2300-8.6460$

H $-0.7820 \quad 0.6396 \quad-7.2550$ 
H $\quad 6.6344 \quad-2.7687 \quad-3.8076$

$\begin{array}{llll}\mathrm{H} & -6.3082 & 5.8977 & -3.4179\end{array}$

H $-3.5127 \quad 2.3188-4.2648$

$\begin{array}{lllll}\mathrm{H} & 8.5669 & 3.1377 & -2.5284\end{array}$

H $5.8497 \quad 1.2337 \quad 4.3606$

H $-0.0467-3.0849-1.8583$

$\begin{array}{lllll}\text { H } & 0.8004 & -0.8467 & -2.4684\end{array}$

$\begin{array}{llll}\mathrm{H} & -0.0550 & -1.1552 & -1.2075\end{array}$

H $-0.0303 \quad 11.9479 \quad-2.2728$

$\begin{array}{llll}\mathrm{H} & -0.4427 & 8.9575 & 7.5071\end{array}$

H $0.8796 \quad-6.2166 \quad-3.9347$

C $-0.2760 \quad-0.1159 \quad 1.0932$

$\begin{array}{llll}\text { O } & -0.4648 & -1.1638 & 0.3635\end{array}$

$\begin{array}{llll}\text { O } & -0.5248 & 0.0046 & 2.2959\end{array}$

H $2.5463 \quad-1.9278 \quad 9.1369$

$\begin{array}{llll}\text { H } & 2.0191 & 0.2636 & 9.0485\end{array}$

H $0.9381 \quad 0.1902-7.2401$

$\begin{array}{llll}\mathrm{H} & -8.4604 & -1.0627 & -5.5745\end{array}$

H $1.7652 \quad 6.7241 \quad-4.3157$

C $-4.3738-5.1846-0.3711$

H $-9.3805-4.6832-1.9452$

H $-10.4440 \quad-3.3242-1.8794$

H $-7.7885-1.1233-1.3474$

$\begin{array}{llll}\mathrm{H} & -9.3693 & -1.5302 & -0.7427\end{array}$

H $-6.4889-2.8358-1.5317$

H $-7.5507-5.1857-2.9805$

H $-7.0564-5.3476-1.2859$

$\begin{array}{llll}\mathrm{H} & -5.2887 & -6.0456 & -2.8924\end{array}$

H $-5.3240 \quad-4.4613-3.6570$

$\begin{array}{llll}\mathrm{H} & -4.3185 & -6.2740 & -0.4972\end{array}$

$\begin{array}{llll}\mathrm{H} & -5.2878 & -4.9587 & 0.1828\end{array}$

H $-3.3645-4.6609-2.2025$

$\begin{array}{llll}\mathrm{H} & -4.3934 & -3.4123 & -1.5678\end{array}$

$\begin{array}{llll}\mathrm{H} & -2.3081 & -1.3778 & 0.1482\end{array}$

H $-3.5271-1.2408 \quad 1.0904$

H -12.2727 $-3.1271 \quad 3.4976$

$\mathrm{H}-12.0107 \quad-1.7272 \quad 2.4351$

$\begin{array}{lll}\mathrm{H}-12.3957 & -3.2997 & 1.7380\end{array}$

H - $10.1647 \quad-4.1955 \quad 2.6100$

H $-9.8188-2.6233 \quad 3.2905$

H - $12.4393-2.1123-1.6422$

$\mathrm{H}-11.4914 \quad-2.3561 \quad-0.4310$

$\begin{array}{llll}\mathrm{H} & -2.3305 & -4.6887 & 0.0927\end{array}$

\section{TS1}

N $-3.3531-4.6015 \quad-0.0723$

$\begin{array}{llll}\text { C } & -4.4885 & -4.2018 & -2.2223\end{array}$

$\begin{array}{llll}\text { C } & -5.6512 & -4.5433 & -3.1613\end{array}$

$\begin{array}{llll}\text { C } & -7.0567 & -4.2832 & -2.6118\end{array}$

$\begin{array}{llll}\mathrm{N} & -7.2386 & -2.8767 & -2.2609\end{array}$

C $-8.4540-2.3226-2.0305$

N $-9.5682-2.9731-2.3750$

$\mathrm{N}-8.5517-1.0613-1.5753$

C $-11.9296 \quad-2.7111 \quad 2.2260$

C $-10.4459-3.0846 \quad 2.1524$

$\begin{array}{llll}\text { C } & -9.6696 & -2.4985 & 0.9662\end{array}$

$\begin{array}{lllll}\text { O } & -8.6337 & -3.0659 & 0.5807\end{array}$

O $-10.1525 \quad-1.4064 \quad 0.4447$

O $-11.9590 \quad-2.0124 \quad-1.3685$ $\begin{array}{llll}\text { O } & -4.2303 & -1.6552 & 0.3296\end{array}$

C $6.3144-8.4135-1.8440$

C $5.1782 \quad-7.8992 \quad-0.9749$

O $4.0593-8.4063-0.9622$

N $5.4817 \quad-6.8350 \quad-0.1687$

C $4.5655-6.45210 .8965$

C $3.5486 \quad-5.3800 \quad 0.4926$

$\begin{array}{llll}\text { O } & 3.8686 & -4.3830 & -0.1716\end{array}$

C $5.5166-5.8586 \quad 1.9625$

C $6.6419 \quad-5.2343 \quad 1.1221$

C $6.8054-6.2055-0.0545$

N $\quad 2.3217 \quad-5.5101 \quad 1.0342$

C $1.3458-4.4534 \quad 0.9220$

C $-0.0043-4.9218 \quad 1.4510$

$\begin{array}{llll}\mathrm{O} & -0.1783 & -6.0569 & 1.8807\end{array}$

N $-0.9687-3.9573 \quad 1.3859$

$\begin{array}{llll}\text { C } & -2.2233 & -4.1141 & 2.1002\end{array}$

$\begin{array}{llll}\text { C } & -3.4488 & -4.5238 & 1.2748\end{array}$

$\begin{array}{llll}\mathrm{O} & -4.5180 & -4.6978 & 1.8746\end{array}$

$\begin{array}{llll}\text { C } & -2.5081 & -2.7941 & 2.9011\end{array}$

$\begin{array}{llll}\text { O } & -3.8659 & -2.3506 & 2.9005\end{array}$

C $7.8993-4.5085-6.9131$

C 7.4978 -5.2233 $\quad-5.6183$

C $7.0372-4.3600-4.4502$

O $6.8141 \quad-3.0863 \quad-4.7747$

$\begin{array}{lllll}\text { O } & 6.8643 & -4.8124 & -3.3290\end{array}$

C $0.0521-5.0567-4.0565$

$\begin{array}{llll}\text { C } & -0.2801 & -4.0843 & -5.2065\end{array}$

$\begin{array}{llll}\text { O } & 0.2287 & -4.1728 & -6.3193\end{array}$

$\begin{array}{llll}\text { C } & 0.0625 & -4.4133 & -2.6524\end{array}$

$\begin{array}{llll}\text { C } & 1.0213 & -3.2267 & -2.5441\end{array}$

$\begin{array}{llll}\text { O } & -1.2881 & -4.0317 & -2.3195\end{array}$

N -1.2177 $-3.1193-4.9216$

$\begin{array}{llll}\mathrm{C} & -1.8291 & -2.3314 & -5.9737\end{array}$

C $-1.2573-0.9006-6.1100$

$\begin{array}{llll}\mathrm{O} & -1.6063 & -0.0072 & -5.3134\end{array}$

C $-3.3414-2.2053-5.7022$

$\begin{array}{lllll}\mathrm{O} & -3.6021 & -1.5799 & -4.4478\end{array}$

$\begin{array}{llll}\mathrm{N} & -0.4675 & -0.6308 & -7.1637\end{array}$

$\begin{array}{llll}\text { C } & 0.0017 & 0.7289 & -7.4660\end{array}$

$\begin{array}{llll}\text { C } & -8.0851 & 0.5051 & -4.8633\end{array}$

$\begin{array}{llll}\mathrm{C} & -6.6597 & 0.0804 & -4.5017\end{array}$

$\begin{array}{llll}\mathrm{N} & -6.0074 & 1.0472 & -3.6079\end{array}$

$\begin{array}{llll}\mathrm{C} & -5.3421 & 0.7509 & -2.4864\end{array}$

$\mathrm{N}-5.0305-0.5228 \quad-2.1861$

$\mathrm{N}-5.0353 \quad 1.7253 \quad-1.6125$

$\begin{array}{llll}\text { C } & -5.0260 & 6.2940 & -2.6141\end{array}$

C $-4.6953 \quad 5.0026-3.3619$

$\begin{array}{lllll}\text { O } & -5.4380 & 4.0138 & -3.2492\end{array}$

C $-4.6562 \quad 6.0567 \quad-1.1292$

$\begin{array}{llll}\text { C } & -3.1651 & 6.1949 & -0.8570\end{array}$

$\mathrm{N}-2.4822 \quad 5.1156 \quad-0.4507$

$\begin{array}{lllll}\mathrm{O} & -2.6404 & 7.3243 & -0.9748\end{array}$

N $-3.5168 \quad 4.9604 \quad-4.0175$

$\begin{array}{llll}\text { C } & -2.7825 & 3.7120 & -4.2878\end{array}$

C $-1.4036 \quad 3.8028-3.6210$

C $-0.8204 \quad 2.4549-3.2292$

$\begin{array}{llll}\mathrm{C} & -1.6986 & 1.7189 & -2.2351\end{array}$

$\begin{array}{llll}\mathrm{N} & -1.8366 & 0.4090 & -2.4716\end{array}$

O $-2.2964 \quad 2.3015-1.3005$
O $-1.7363-1.7984 \quad-0.8873$

$\begin{array}{llll}\text { C } & -0.7713 & 7.6223 & 7.6802\end{array}$

$\begin{array}{llll}\text { C } & 0.2023 & 6.5728 & 7.1248\end{array}$

$\begin{array}{llll}\mathrm{N} & -0.4173 & 5.5556 & 6.2600\end{array}$

C $-0.2622 \quad 5.4316 \quad 4.9283$

$\begin{array}{llll}\mathrm{N} & 0.2761 & 6.4093 & 4.1917\end{array}$

$\begin{array}{llll}\mathrm{N} & -0.6511 & 4.3135 & 4.3018\end{array}$

C $\quad 1.1949 \quad 11.9830 \quad-0.2237$

C $\quad 0.8152 \quad 10.6132 \quad-0.7811$

$\begin{array}{llll}\mathrm{N} & -0.0292 & 9.8410 & 0.1366\end{array}$

$\begin{array}{llll}\text { C } & 0.2987 & 8.6521 & 0.6775\end{array}$

$\begin{array}{llll}\mathrm{N} & -0.6927 & 7.8355 & 1.0761\end{array}$

$\begin{array}{llll}\mathrm{N} & 1.5610 & 8.3039 & 0.9280\end{array}$

C $\quad \begin{array}{llll}1.9956 & 6.5063 & -2.5707\end{array}$

C $2.6331 \quad 5.2053 \quad-3.0284$

$\begin{array}{lllll}\text { O } & 2.1211 & 4.1004 & -2.8090\end{array}$

$\begin{array}{lllll}\text { C } & 0.4825 & 6.4387 & -2.4828\end{array}$

$\begin{array}{lllll}\text { O } & 0.0003 & 7.6869 & -2.0065\end{array}$

N $3.8115 \quad 5.3684 \quad-3.6796$

C $4.7753 \quad 4.2985-3.8540$

C $5.9864 \quad 4.6105-2.9643$

$\begin{array}{lllll}\text { O } & 6.2728 & 5.7850 & -2.7263\end{array}$

N $6 \begin{array}{llll}6.6787 & 3.5371 & -2.5298\end{array}$

C $7.8204 \quad 3.6852-1.6291$

C $7.5981 \quad 3.0083-0.2638$

C $7.7177 \quad 1.4767 \quad-0.3621$

$\begin{array}{llll}\text { C } & 6.2720 & 3.4511 & 0.3716\end{array}$

C $7.7222 \quad 0.7713 \quad 0.9957$

$\begin{array}{llll}\text { C } & 2.3518 & 0.2368 & 8.9832\end{array}$

$\begin{array}{llll}\text { C } & 2.0877 & 0.6960 & 7.5438\end{array}$

$\begin{array}{llll}\text { C } & 0.5906 & 0.6089 & 7.1997\end{array}$

$\begin{array}{llll}\text { C } & 2.9554 & -0.0987 & 6.5633\end{array}$

$\begin{array}{llll}\text { C } & 0.2160 & 1.2119 & 5.8419\end{array}$

C $\quad 1.4498 \quad-3.4639 \quad 8.8908$

C $\quad 1.4275 \quad-4.4119 \quad 7.6796$

$\begin{array}{llll}\text { C } & 1.5920 & -3.7039 & 6.3523\end{array}$

$\begin{array}{llll}\text { C } & 0.5499 & -2.9238 & 5.8299\end{array}$

$\begin{array}{llll}\text { C } & 2.7799 & -3.7977 & 5.6176\end{array}$

C $\quad 0.6860 \quad-2.2564 \quad 4.6132$

C $2.9243 \quad-3.1340 \quad 4.3966$

C $1.8796-2.3605 \quad 3.8920$

C $-3.2921 \quad-2.4029 \quad 10.2741$

C $-3.5294 \quad-2.5114 \quad 8.7644$

$\begin{array}{llll}\text { C } & -2.6989 & -1.5408 & 7.9063\end{array}$

$\begin{array}{llll}\text { C } & -2.9216 & -1.8127 & 6.4129\end{array}$

$\begin{array}{llll}\text { C } & -2.9988 & -0.0728 & 8.2322\end{array}$

$\begin{array}{llll}C & 0.3803 & 2.3629 & 1.2010\end{array}$

$\begin{array}{llll}\text { C } & 1.0805 & 4.8559 & 1.0046\end{array}$

$\begin{array}{lllll}\text { O } & 0.6260 & 0.8463 & -0.6604\end{array}$

$\begin{array}{llll}\mathrm{O} & 0.0376 & 5.1770 & 1.7084\end{array}$

$\begin{array}{llll}\text { O } & 1.9572 & 5.6839 & 0.6664\end{array}$

$\begin{array}{llll}\text { C } & 6.9095 & 0.3242 & -4.2470\end{array}$

$\begin{array}{llll}\text { C } & 5.3033 & -1.8814 & 1.3477\end{array}$

$\begin{array}{llll}\text { C } & 2.9846 & -1.1239 & -0.3612\end{array}$

C $4.8224-1.6660-1.9976$

C $3.9583-0.7416-1.4436$

$\begin{array}{llll}\text { C } & 4.0161 & 0.5795 & -1.9879\end{array}$

$\begin{array}{llll}\mathrm{N} & 3.1283 & 1.5271 & -1.6478\end{array}$

$\begin{array}{llll}\mathrm{N} & 5.0047 & 0.9102 & -2.8599\end{array}$

C $5.8065-0.0659-3.3025$ 
N $5.7293 \quad-1.3560 \quad-2.9506$

S $3.1071 \quad 1.1212 \quad 2.8587$

C $2.4856 \quad 0.5557 \quad 1.3665$

$\begin{array}{llll}\text { N } & 3.2759 & -0.4259 & 0.9251\end{array}$

$\begin{array}{llll}\text { C } & 4.3850 & -0.7712 & 1.7286\end{array}$

$\begin{array}{llll}C & 4.4494 & -0.0070 & 2.8517\end{array}$

C $5.4291 \quad-0.1006 \quad 3.9793$

$\begin{array}{llll}\text { C } & 0.5946 & 0.9693 & 0.6006\end{array}$

$\begin{array}{llll}\text { C } & 1.2321 & 3.4257 & 0.5152\end{array}$

H $3.1123 \quad 2.3983-2.1689$

H $\quad 2.2180 \quad 1.2679-1.2381$

H $-0.6850 \quad 2.5822 \quad 1.0580$

$\begin{array}{llll}\mathrm{H} & 0.5509 & 2.3294 & 2.2796\end{array}$

$\begin{array}{llll}\text { H } & 7.8119 & 0.5715 & -3.6748\end{array}$

H $6.6288 \quad 1.2038$-4.8287

H $7.1583 \quad-0.5040-4.9124$

H $4.7482-2.7741 \quad 1.0515$

H $5.9460 \quad-2.1357 \quad 2.1913$

H $5.9428 \quad-1.6047 \quad 0.5050$

H $5.0879 \quad-0.8232 \quad 4.7278$

H $1.0203 \quad 3.4376 \quad-0.5606$

H $4.7977-2.7060-1.6769$

H $1.9563 \quad-0.8598 \quad-0.6085$

H $6.4109 \quad-0.4138 \quad 3.6169$

$\begin{array}{llll}\text { H } & 2.2949 & 3.1758 & 0.5839\end{array}$

H $3.0379 \quad-2.1982-0.1824$

H $-0.1172-1.6467 \quad 4.2153$

H $1.9791 \quad-1.8452 \quad 2.9439$

$\begin{array}{llll}\mathrm{H} & -0.3795 & -2.8390 & 6.3867\end{array}$

H $3.5972-4.4016 \quad 6.0035$

H $3.8537 \quad-3.2216 \quad 3.8403$

$\begin{array}{llll}\text { H } & 0.4799 & -4.9643 & 7.6761\end{array}$

H $2.2198 \quad-5.1598 \quad 7.7929$

H $\quad 0.6531 \quad-2.7163 \quad 8.8183$

H $-3.3061-3.5357 \quad 8.4396$

$\begin{array}{llll}\mathrm{H} & -4.5945 & -2.3512 & 8.5463\end{array}$

$\begin{array}{llll}\mathrm{H} & -4.0625 & 0.1461 & 8.0781\end{array}$

$\begin{array}{llll}\text { H } & -2.7509 & 0.1770 & 9.2679\end{array}$

$\begin{array}{llll}\mathrm{H} & -2.4237 & 0.5947 & 7.5818\end{array}$

H $-1.6371 \quad-1.7250 \quad 8.1310$

H $-3.9593-1.6125 \quad 6.1236$

H $-2.2799-1.1820 \quad 5.7884$

$\begin{array}{llll}\mathrm{H} & -2.7107 & -2.8591 & 6.1627\end{array}$

H $-0.6775 \quad 3.4368 \quad 4.8057$

H $-0.5291 \quad 4.2907 \quad 3.2806$

$\begin{array}{llll}\mathrm{H} & 0.2736 & 7.3628 & 4.5135\end{array}$

$\begin{array}{llll}\mathrm{H} & 0.3812 & 6.2046 & 3.1887\end{array}$

H $-0.9341 \quad 4.8167 \quad 6.7155$

$\begin{array}{llll}\mathrm{H} & 1.0075 & 7.0437 & 6.5553\end{array}$

H $\quad 0.6850 \quad 6.0393 \quad 7.9495$

H $-1.2464 \quad 8.1927 \quad 6.8767$

H $-1.5592 \quad 7.1519 \quad 8.2750$

H $-0.4124 \quad 6.8879 \quad 1.3740$

H $-1.5309 \quad 7.8429 \quad 0.4944$

$\begin{array}{llll}\mathrm{H} & -1.0230 & 10.0112 & 0.0707\end{array}$

$\begin{array}{llll}\text { H } & 2.2968 & 8.9477 & 0.6842\end{array}$

H $1.7780 \quad 7.2692 \quad 0.9288$

$\begin{array}{llll}\mathrm{H} & 0.2743 & 10.7078 & -1.7276\end{array}$

H $1.6972 \quad 10.0123 \quad-1.0114$

H $1.7982 \quad 12.5330 \quad-0.9513$
H $1.7711 \quad 11.88640 .7012$

H $7.1462-8.7715-1.2284$

H $7.5715 \quad-6.96120 .1632$

H $7.0724 \quad-5.6950 \quad-0.9834$

H $7.5703 \quad-5.1043 \quad 1.6842$

H $\quad 6.3282 \quad-4.2635 \quad 0.7401$

H $5.9040 \quad-6.6738 \quad 2.5819$

H $\quad \begin{array}{llll}5.0136 & -5.1458 & 2.6220\end{array}$

H $\quad 4.0335 \quad-7.3403 \quad 1.2467$

H $\quad 2.0070 \quad-6.3631 \quad 1.4822$

H $\quad 1.2488-4.1340 \quad-0.1161$

H $1.6563 \quad-3.5719 \quad 1.4880$

H $-0.6344 \quad-2.9900 \quad 1.2371$

H $-2.1030-4.9440 \quad 2.8036$

H $-1.9194-1.9783 \quad 2.4769$

H $-2.1641 \quad-2.9428 \quad 3.9275$

H $-4.4263-3.1542 \quad 2.9275$

H $-4.7405-1.1256-2.9564$

H $-4.6272-0.7074-1.2646$

H $-4.1073 \quad 1.6769-1.1757$

H $-5.2986 \quad 2.6622 \quad-1.9092$

H $-6.05292 .0367 \quad-3.8373$

H $-6.6816-0.8821 \quad-3.9883$

H $-6.0445-0.0426 \quad-5.4001$

H $-8.1024 \quad 1.4911 \quad-5.3392$

H $-8.7100 \quad 0.5271 \quad-3.9672$

H $-1.2913 \quad 0.0265 \quad-3.2321$

H $-2.0077 \quad-0.2611-1.7144$

H $-0.6626 \quad 1.8254-4.1082$

$\begin{array}{llll}\mathrm{H} & 0.1532 & 2.6037 & -2.7521\end{array}$

H $-0.6987 \quad 4.3252 \quad-4.2737$

H $-1.5103 \quad 4.3996-2.7147$

H $-2.7056 \quad 3.5361 \quad-5.3655$

H $-2.9713 \quad 5.8118-4.0332$

H $-4.4856 \quad 7.1632-2.9982$

H $-5.0407 \quad 5.0873 \quad-0.8031$

$\begin{array}{lllll}\mathrm{H} & -5.1335 & 6.8271 & -0.5167\end{array}$

H $-1.5155 \quad 5.2208-0.1608$

H $-2.7752 \quad 4.1518-0.6046$

H $7.9284 \quad-0.2971 \quad 0.8824$

H $8.4927 \quad 1.1900 \quad 1.6529$

H $\quad 6.7580 \quad 0.8723 \quad 1.5007$

H $\quad 6.8931 \quad 1.0827 \quad-0.9688$

H $8.6441 \quad 1.2302 \quad-0.8971$

H $\begin{array}{llll}5.4162 & 3.0209 & -0.1595\end{array}$

H $\quad 6.2051 \quad 3.1334 \quad 1.4157$

H $\quad 6.1661 \quad 4.5401 \quad 0.3432$

H $8.4223 \quad 3.3575 \quad 0.3753$

H $7.9666 \quad 4.7594-1.5060$

H $\quad 6.2448 \quad 2.6238 \quad-2.6604$

H $5.1139 \quad 4.2637 \quad-4.8968$

H $4.3209 \quad 3.3362 \quad-3.6255$

H $4.2647 \quad 6.2722 \quad-3.5893$

H $2.3835 \quad 6.7008$ - 1.5640

H $\quad 0.0632 \quad 6.2112 \quad-3.4729$

H $\quad 0.2102 \quad 5.6136-1.8142$

H $-0.9361 \quad 7.5697-1.7635$

H $\quad 6.6948 \quad-7.6170 \quad-2.4930$

H $\quad 5.9338 \quad-9.2352 \quad-2.4494$

H $8.1880 \quad-5.2401 \quad-7.6730$
H $7.0722 \quad-3.9112-7.3041$

H $8.7448 \quad-3.8347 \quad-6.7489$

H $6.6738 \quad-5.9229-5.8094$

H $\quad 8.3158 \quad-5.8427 \quad-5.2353$

H $0.6006 \quad 0.6368 \quad 4.9972$

H $-0.8725 \quad 1.2494 \quad 5.7147$

H $\quad 0.6279 \quad 2.2281 \quad 5.7544$

H $2.7960 \quad 0.2152 \quad 5.5300$

H $\quad 4.0174 \quad 0.0295 \quad 6.7981$

H $2.7253-1.1662 \quad 6.6159$

H $0.2804 \quad-0.4411 \quad 7.2204$

$\begin{array}{llll}\mathrm{H} & 0.0211 & 1.1137 & 7.9912\end{array}$

H $2.3769 \quad 1.7573 \quad 7.4717$

$\begin{array}{llll}\mathrm{H} & 1.7658 & 0.8226 & 9.7007\end{array}$

H $3.4093 \quad 0.3511 \quad 9.2431$

H $\quad \begin{array}{llll}1.3105 & -4.0183 & 9.8235\end{array}$

H $-3.8480 \quad-3.1747 \quad 10.8143$

H $-2.2297 \quad-2.5284 \quad 10.5150$

H $-3.6093-1.4331 \quad 10.6691$

H $0.8397 \quad-2.4911 \quad-3.3321$

H $0.8934-2.7177 \quad-1.5855$

H $2.0586 \quad-3.5661 \quad-2.6087$

H $\quad 0.3800 \quad-5.1911-1.9450$

H $-0.6909-5.8621 \quad-4.0725$

H $-1.6369-3.1309-3.9942$

H $-1.6728-2.8690 \quad-6.9142$

H $-3.0447-0.7720-4.4526$

H $-3.8156-1.6509-6.5249$

H $-3.7779-3.2064-5.6636$

H $-0.1065-1.4134-7.6935$

$\begin{array}{lllll}\mathrm{H} & 0.1798 & 0.8098 & -8.5391\end{array}$

H $-0.7727 \quad 1.4334-7.1628$

H $\quad 6.4635-2.5743 \quad-3.9819$

H $-6.0988 \quad 6.4726-2.6999$

H $-3.3829 \quad 2.9063 \quad-3.8644$

H $\quad 8.7187 \quad 3.2736-2.1061$

H $\begin{array}{llll}5.5518 & 0.8628 & 4.4800\end{array}$

H $-1.3162-3.2075-1.7670$

H $-0.9715-1.5364-0.2900$

H $-2.5293-1.8123 \quad-0.3157$

H $\quad 0.3042 \quad 12.5792 \quad-0.0046$

$\begin{array}{llll}\mathrm{H} & -0.2278 & 8.3174 & 8.3255\end{array}$

H $\quad 1.0260 \quad-5.4951 \quad-4.2812$

$\begin{array}{llll}\text { C } & 0.0166 & -0.2079 & 1.4541\end{array}$

$\begin{array}{llll}\text { O } & 0.0610 & -1.3593 & 0.8784\end{array}$

$\begin{array}{llll}\text { O } & -0.4293 & 0.0370 & 2.5855\end{array}$

H $2.4031 \quad-2.9337 \quad 8.9462$

H $2.0920 \quad-0.8136 \quad 9.1282$

H $\quad 0.9241 \quad 0.9648 \quad-6.9258$

H $-8.5116 \quad-0.2154 \quad-5.5662$

H $\quad 2.2967 \quad 7.3366 \quad-3.2175$

$\begin{array}{lllll}\text { C } & -4.5051 & -4.9714 & -0.8998\end{array}$

H $-9.5463-3.9806-2.3425$

H $-10.4819-2.5465-2.1244$

H $-7.6546-0.6683-1.3154$

H $-9.2619-1.0393-0.7432$

H $-6.5126-2.4762-1.6832$

H $-7.7822 \quad-4.5308-3.3917$

H $-7.2677-4.9185-1.7413$

H $\quad-5.5967 \quad-5.6038 \quad-3.4334$ 
H $-5.5303-3.9707-4.0877$

H $-4.4980-6.0513-1.0972$

H $-5.3977-4.7683-0.3006$

H $-3.5454-4.4026-2.7362$

H $-4.4677-3.1254-2.0197$

$\begin{array}{llll}\mathrm{H} & -4.4103 & -2.5830 & 0.1256\end{array}$

H $-4.1874-1.6701 \quad 1.3232$

H -12.4058 -3.17293 .0965$

H -12.0497 $-1.6272 \quad 2.2972$

H -12.4646 $-3.0414 \quad 1.3297$

H $-10.3061 \quad-4.1697 \quad 2.1224$

H $-9.9293-2.7428 \quad 3.0598$

H -12.3749 $-1.2119-1.7105$

H $-11.4639-1.7240-0.5544$

H $-2.4395 \quad-4.5418-0.5137$

\section{Int1}

$\begin{array}{llll}\mathrm{N} & -3.3934 & -4.6139 & 0.2890\end{array}$

$\begin{array}{llll}\mathrm{C} & -4.4868 & -4.3511 & -1.8921\end{array}$

$\begin{array}{llll}\text { C } & -5.5689 & -4.8299 & -2.8685\end{array}$

$\begin{array}{llll}\text { C } & -7.0154 & -4.5847 & -2.4304\end{array}$

N $-7.2947-3.1556-2.3219$

$\begin{array}{llll}\text { C } & -8.5431 & -2.6581 & -2.1543\end{array}$

N $-9.6118-3.4257 \quad-2.3791$

N $-8.7226-1.3531-1.8816$

$\begin{array}{llll}\mathrm{C}-11.9935 & -2.5133 & 2.1788\end{array}$

$\begin{array}{lll}\text { C }-10.5007 & -2.8485 & 2.1382\end{array}$

$\begin{array}{llll}\text { C } & -9.7581 & -2.4185 & 0.8666\end{array}$

$\begin{array}{llll}\text { O } & -8.6829 & -2.9679 & 0.5791\end{array}$

O $-10.3140 \quad-1.4669 \quad 0.1703$

$\begin{array}{llll}\mathrm{O}-12.0657 & -2.5026 & -1.4927\end{array}$

$\begin{array}{llll}\text { O } & -3.6853 & -1.2005 & 0.1530\end{array}$

$\begin{array}{llll}\text { C } & 6.3194 & -8.6021 & -0.9029\end{array}$

C $5.1096 \quad-8.0035-0.1923$

$\begin{array}{lllll}\text { O } & 4.0050 & -8.5394 & -0.1988\end{array}$

$\begin{array}{llll}\mathrm{N} & 5.3245 & -6.8308 & 0.4912\end{array}$

$\begin{array}{llll}\text { C } & 4.3370 & -6.3651 & 1.4593\end{array}$

C $3.4161 \quad-5.2500 \quad 0.9463$

$\begin{array}{llll}\text { O } & 3.8560 & -4.2910 & 0.2902\end{array}$

C $5.2243 \quad-5.7835 \quad 2.5855$

$\begin{array}{llll}\text { C } & 6.4291 & -5.2064 & 1.8244\end{array}$

$\begin{array}{llll}\text { C } & 6.6319 & -6.1744 & 0.6459\end{array}$

$\begin{array}{llll}\mathrm{N} & 2.1407 & -5.3035 & 1.3676\end{array}$

$\begin{array}{llll}\text { C } & 1.2115 & -4.2043 & 1.2025\end{array}$

$\begin{array}{llll}\text { C } & -0.1261 & -4.5925 & 1.8257\end{array}$

$\begin{array}{llll}\text { O } & -0.2619 & -5.6460 & 2.4436\end{array}$

$\begin{array}{llll}\mathrm{N} & -1.1192 & -3.6824 & 1.6318\end{array}$

$\begin{array}{llll}\text { C } & -2.3621 & -3.7717 & 2.3785\end{array}$

$\begin{array}{llll}\text { C } & -3.5817 & -4.2371 & 1.5728\end{array}$

$\begin{array}{llll}\text { O } & -4.6904 & -4.2405 & 2.1214\end{array}$

$\begin{array}{llll}\text { C } & -2.6762 & -2.4250 & 3.1187\end{array}$

$\begin{array}{llll}\text { O } & -3.9063 & -1.8011 & 2.7407\end{array}$

C $8.0344 \quad-5.2005-6.2829$

C $7.9301 \quad-5.7247 \quad-4.8450$

C $7.2584-4.8045-3.8351$

O $7.2961 \quad-3.5204-4.1866$

$\begin{array}{llll}\text { O } & 6.7614 & -5.2071 & -2.7935\end{array}$

C $0.1216-5.4635-3.5706$

C $-0.1993-4.5100-4.7335$ $\begin{array}{lllll}\text { O } & 0.1886 & -4.7161 & -5.8813\end{array}$

C $\quad 0.7331-4.8257 \quad-2.3228$

C $2.0309-4.0794-2.6226$

$\begin{array}{llll}\mathrm{O} & -0.2401 & -3.9855 & -1.6830\end{array}$

N $-1.0096-3.4365-4.4474$

C $-1.7125-2.7424 \quad-5.5130$

C $-1.1800-1.3339-5.8819$

O $-1.6378-0.3190 \quad-5.3275$

C $-3.1983-2.6067-5.1502$

$\begin{array}{lllll}\mathrm{O} & -3.3753 & -1.8508 & -3.9546\end{array}$

$\mathrm{N}-0.2964-1.2783 \quad-6.9000$

C $0.1703-0.0295 \quad-7.5166$

$\begin{array}{llll}\text { C } & -7.9752 & 0.0068 & -5.0934\end{array}$

$\begin{array}{llll}\text { C } & -6.5293 & -0.2922 & -4.6937\end{array}$

$\begin{array}{llll}\mathrm{N} & -6.0022 & 0.7265 & -3.7797\end{array}$

$\begin{array}{llll}\text { C } & -5.5409 & 0.5123 & -2.5446\end{array}$

$\begin{array}{llll}\mathrm{N} & -5.2565 & -0.7402 & -2.1286\end{array}$

N $-5.4324 \quad 1.5514 \quad-1.6989$

$\begin{array}{llll}\text { C } & -4.9482 & 5.9798 & -3.3359\end{array}$

$\begin{array}{llll}C & -4.5837 & 4.6001 & -3.8679\end{array}$

$\begin{array}{lllll}\mathrm{O} & -5.3181 & 3.6257 & -3.6394\end{array}$

C $-4.6205 \quad 6.0674-1.8266$

C $-3.1373 \quad 6.2377 \quad-1.5054$

N $-2.4945 \quad 5.1740 \quad-1.0258$

$\begin{array}{lllll}\mathrm{O} & -2.5946 & 7.3586 & -1.6634\end{array}$

$\mathrm{N}-3.4116 \quad 4.5040 \quad-4.5295$

$\begin{array}{llll}\text { C } & -2.6761 & 3.2470 & -4.7030\end{array}$

C $-1.3824 \quad 3.2762 \quad-3.8511$

C $-1.0676 \quad 1.9804 \quad-3.1102$

$\begin{array}{llll}\text { C } & -2.0971 & 1.6151 & -2.0574\end{array}$

N $-2.2489 \quad 0.3073 \quad-1.8242$

$\begin{array}{lllll}\mathrm{O} & -2.7832 & 2.4604 & -1.4384\end{array}$

$\begin{array}{llll}\text { O } & -0.0527 & -1.3721 & -2.0523\end{array}$

$\begin{array}{llll}C & -0.9274 & 8.2786 & 6.8811\end{array}$

C $-0.2765 \quad 6.9303 \quad 6.5493$

$\begin{array}{llll}\mathrm{N} & -0.9564 & 6.1929 & 5.4764\end{array}$

$\begin{array}{llll}\mathrm{C} & -0.4927 & 5.9385 & 4.2367\end{array}$

$\begin{array}{llll}\mathrm{N} & 0.5559 & 6.5860 & 3.7299\end{array}$

$\mathrm{N}-1.1106 \quad 5.0224 \quad 3.4815$

C $1.2358 \quad 11.8627-1.3546$

C $1.5892 \quad 10.4017 \quad-1.0880$

$\begin{array}{lllll}\mathrm{N} & 0.4530 & 9.6916 & -0.4974\end{array}$

$\begin{array}{lllll}\text { C } & 0.5384 & 8.4911 & 0.1058\end{array}$

$\begin{array}{llll}\mathrm{N} & -0.5807 & 7.8322 & 0.4372\end{array}$

$\begin{array}{llll}\mathrm{N} & 1.7235 & 8.0042 & 0.4879\end{array}$

C $2.0711 \quad 6.1860 \quad-3.1494$

C $2.7456 \quad 4.8527 \quad-3.4310$

$\begin{array}{llll}\mathrm{O} & 2.2860 & 3.7790 & -3.0312\end{array}$

C $\quad 0.5756 \quad 6.0918-2.8951$

$\begin{array}{lllll}\mathrm{O} & 0.1154 & 7.3895 & -2.5457\end{array}$

N $3.9007 \quad 4.9582 \quad-4.1419$

C $4.8949 \quad 3.9014 \quad-4.1754$

C $6.0469 \quad 4.2803 \quad-3.2311$

$\begin{array}{llll}\mathrm{O} & 6.2961 & 5.4721 & -3.0377\end{array}$

N $6.7393 \quad 3.2436 \quad-2.7160$

C $7.8626 \quad 3.4600-1.8077$

C $7.5478 \quad 3.1082-0.3400$

C $7.5899 \quad 1.5902 \quad-0.0954$

$\begin{array}{llll}\text { C } & 6.2208 & 3.7371 & 0.1091\end{array}$

C $7.4610 \quad 1.2069 \quad 1.3797$
$\begin{array}{llll}C & 2.1383 & 1.0453 & 8.9551\end{array}$

$\begin{array}{llll}C & 1.8616 & 1.4755 & 7.5075\end{array}$

$\begin{array}{llll}\text { C } & 0.3673 & 1.3436 & 7.1645\end{array}$

$\begin{array}{llll}\text { C } & 2.7428 & 0.6747 & 6.5457\end{array}$

$\begin{array}{llll}\text { C } & -0.0250 & 1.8758 & 5.7805\end{array}$

$\begin{array}{llll}\text { C } & 1.2264 & -2.6423 & 9.1944\end{array}$

$\begin{array}{llll}\text { C } & 1.1839 & -3.6856 & 8.0648\end{array}$

$\begin{array}{llll}\text { C } & 1.3752 & -3.0894 & 6.6867\end{array}$

$\begin{array}{llll}\text { C } & 0.3502 & -2.3337 & 6.0986\end{array}$

C $\quad 2.5637 \quad-3.2662 \quad 5.9683$

C $0.5023 \quad-1.7706 \quad 4.8340$

$\begin{array}{llll}\text { C } & 2.7209 & -2.7127 & 4.6938\end{array}$

$\begin{array}{llll}\text { C } & 1.6899 & -1.9655 & 4.1261\end{array}$

C $-3.5430 \quad-1.4499 \quad 10.3599$

C $-3.7502-1.5805 \quad 8.8492$

$\begin{array}{llll}\text { C } & -2.8970 & -0.6268 & 7.9946\end{array}$

$\begin{array}{llll}\text { C } & -3.0787 & -0.9366 & 6.5045\end{array}$

$\begin{array}{llll}\text { C } & -3.2078 & 0.8477 & 8.2791\end{array}$

$\begin{array}{llll}\text { C } & 0.4482 & 2.2615 & 0.8922\end{array}$

$\begin{array}{llll}C & 0.9423 & 4.7212 & 0.9121\end{array}$

$\begin{array}{lllll}\text { O } & 1.0290 & 0.7358 & -0.8738\end{array}$

$\begin{array}{llll}\text { O } & -0.2819 & 5.0869 & 0.7972\end{array}$

$\begin{array}{llll}\text { O } & 1.7984 & 5.4283 & 1.5288\end{array}$

C $7.3010-0.1340-4.0031$

$\begin{array}{llll}\text { C } & 5.2418 & -1.6520 & 1.5444\end{array}$

C $3.0433-1.2706-0.3689$

C $5.0041-1.9275-1.7906$

$\begin{array}{llll}\text { C } & 4.1250 & -0.9507 & -1.3649\end{array}$

$\begin{array}{llll}\text { C } & 4.2747 & 0.3457 & -1.9561\end{array}$

N $3.4246 \quad 1.3438$ - 1.6941

N $5.3272 \quad 0.5844-2.7872$

C $6.1285-0.4339-3.1110$

$\begin{array}{llll}\text { N } & 5.9940 & -1.6951 & -2.6794\end{array}$

$\begin{array}{llll}\text { S } & 2.6984 & 1.2387 & 2.6898\end{array}$

$\begin{array}{llll}\text { C } & 2.2451 & 0.5085 & 1.2045\end{array}$

N $3.1403 \quad-0.4278 \quad 0.8621$

C $4.2068 \quad-0.6001 \quad 1.7630$

$\begin{array}{llll}\text { C } & 4.1225 & 0.2435 & 2.8269\end{array}$

C $5.0247 \quad 0.3165 \quad 4.0180$

$\begin{array}{llll}\text { C } & 0.9228 & 0.8420 & 0.4713\end{array}$

$\begin{array}{llll}\text { C } & 1.3475 & 3.3823 & 0.3401\end{array}$

H $3.4920 \quad 2.1880 \quad-2.2501$

H $2.4411 \quad 1.1325 \quad-1.3359$

$\begin{array}{llll}\mathrm{H} & -0.5606 & 2.3830 & 0.4891\end{array}$

$\begin{array}{llll}\mathrm{H} & 0.3552 & 2.3225 & 1.9815\end{array}$

H $8.1827 \quad 0.0813 \quad-3.3875$

H $7.1013 \quad 0.7402-4.6243$

H $7.5408-0.9951 \quad-4.6289$

H $4.7821 \quad-2.6119 \quad 1.2982$

H $5.8338 \quad-1.7742 \quad 2.4515$

H $5.9159 \quad-1.3920 \quad 0.7247$

H $4.6772 \quad-0.3603 \quad 4.8042$

H $1.2634 \quad 3.4092 \quad-0.7514$

H $4.9293-2.9451 \quad-1.4126$

H $2.0515 \quad-1.0898 \quad-0.7678$

$\begin{array}{llll}\mathrm{H} & 6.0437 & 0.0404 & 3.7423\end{array}$

H $\quad 2.4000 \quad 3.2170 \quad 0.5779$

H $3.1281-2.3103-0.0520$

H $-0.2835-1.1768 \quad 4.3842$

H $1.7934-1.5287 \quad 3.1412$ 
H $-0.5804 \quad-2.1936 \quad 6.6397$

H $3.3675 \quad-3.8526 \quad 6.4057$

H $3.6467 \quad-2.8693 \quad 4.1460$

H $\quad 0.2197 \quad-4.2072 \quad 8.0973$

$\begin{array}{llll}\text { H } & 1.9523 & -4.4455 & 8.2436\end{array}$

H $0.4568-1.8773 \quad 9.0516$

H $-3.5241-2.6107 \quad 8.5455$

H $-4.8097-1.4197 \quad 8.6059$

$\begin{array}{llll}\mathrm{H} & -4.2677 & 1.0597 & 8.0924\end{array}$

H $-2.9888 \quad 1.1211 \quad 9.3153$

H $-2.6166 \quad 1.5030 \quad 7.6309$

H $-1.8417 \quad-0.8032 \quad 8.2544$

$\begin{array}{llll}\mathrm{H} & -4.1029 & -0.7226 & 6.1783\end{array}$

$\begin{array}{llll}\mathrm{H} & -2.4011 & -0.3447 & 5.8804\end{array}$

$\begin{array}{llll}\mathrm{H} & -2.8880 & -1.9948 & 6.3004\end{array}$

H $-1.6464 \quad 4.2847 \quad 3.9123$

H $-0.8999 \quad 4.9665 \quad 2.4728$

H $\quad 0.8957 \quad 7.4262 \quad 4.1671$

H $1.0443 \quad 6.2070 \quad 2.8913$

H $-1.8556 \quad 5.7912 \quad 5.7009$

H $\quad 0.7693 \quad 7.0545 \quad 6.2582$

$\begin{array}{llll}\mathrm{H} & -0.2700 & 6.2857 & 7.4343\end{array}$

H $-0.9164 \quad 8.9494 \quad 6.0171$

H $-1.9666 \quad 8.1455 \quad 7.1955$

H $-0.4583 \quad 6.8448 \quad 0.7181$

H $-1.3921 \quad 7.9636-0.1678$

$\begin{array}{lllll}\mathrm{H} & -0.4545 & 9.9147 & -0.8831\end{array}$

$\begin{array}{llll}\mathrm{H} & 2.5713 & 8.4528 & 0.1864\end{array}$

H $1.7885 \quad 7.0461 \quad 0.8704$

H $1.9037 \quad 9.8951 \quad-2.0091$

H $\quad 2.4217 \quad 10.3707 \quad-0.3779$

H $2.0952 \quad 12.3781 \quad-1.7894$

$\begin{array}{lllll}\mathrm{H} & 0.9551 & 12.3718 & -0.4291\end{array}$

$\begin{array}{llll}\text { H } & 7.0927 & -8.8900 & -0.1832\end{array}$

H $7.4031 \quad-6.9175 \quad 0.8794$

H $6.9157 \quad-5.6597 \quad-0.2763$

$\begin{array}{llll}\text { H } & 7.3223 & -5.1289 & 2.4497\end{array}$

H $6.1915 \quad-4.2142 \quad 1.4423$

H $5.5363 \quad-6.6019 \quad 3.2418$

H $4.6986 \quad-5.0444 \quad 3.1972$

H $3.7337-7.2173 \quad 1.7803$

H $1.7779-6.0880 \quad 1.9000$

H $1.0504 \quad-3.9535 \quad 0.1530$

H $1.5830 \quad-3.3051 \quad 1.7071$

H $-0.8729-2.7709 \quad 1.2186$

H $-2.2174 \quad-4.5619 \quad 3.1220$

H $-1.8930-1.69912 .8934$

H $-2.6791-2.6096 \quad 4.1959$

H $-4.5795 \quad-2.5147 \quad 2.7503$

H $-4.7844-1.3142-2.8355$

H $-4.8231-0.8341-1.1992$

H $-4.6121 \quad 1.5654-1.0947$

$\begin{array}{llll}\mathrm{H} & -5.5948 & 2.4610 & -2.1264\end{array}$

H $-6.0208 \quad 1.7003-4.0729$

H $-6.4801-1.2584-4.1903$

H $-5.8774-0.3441-5.5728$

$\begin{array}{llll}\mathrm{H} & -8.0630 & 0.9934 & -5.5599\end{array}$

H $-8.6264-0.0372-4.2170$

H $-1.5347 \quad-0.3449-2.1631$

H $-2.7093 \quad 0.0301-0.9572$
$\begin{array}{llll}\mathrm{H} & -0.9589 & 1.1471 & -3.8082\end{array}$

H $-0.1134 \quad 2.0717-2.5746$

H $-0.5223 \quad 3.5360 \quad-4.4751$

H $-1.4890 \quad 4.0695 \quad-3.1100$

H $-2.4619 \quad 3.0821 \quad-5.7625$

H $-2.8779 \quad 5.3562-4.6331$

$\begin{array}{lllll}\mathrm{H} & -4.4291 & 6.7801 & -3.8726\end{array}$

H $-5.0267 \quad 5.1886-1.3190$

H $-5.1152 \quad 6.9521-1.4172$

H $-1.5423 \quad 5.2434-0.6684$

H $-2.8458 \quad 4.2149 \quad-1.0992$

H $7.6101 \quad 0.1327 \quad 1.5227$

H $8.2081 \quad 1.72891 .9882$

H $6.4726 \quad 1.4617 \quad 1.7704$

H $6.7916 \quad 1.1065-0.6713$

H $8.5371 \quad 1.1975 \quad-0.4875$

$\begin{array}{llll}\text { H } & 5.3707 & 3.2101 & -0.3378\end{array}$

H $6.1048 \quad 3.6919 \quad 1.1955$

H $6.1626 \quad 4.7860 \quad-0.1963$

H $\quad 8.3605 \quad 3.5549 \quad 0.2517$

H $\quad 8.1170 \quad 4.5178 \quad-1.8920$

$\begin{array}{llll}\mathrm{H} & 6.3247 & 2.3113 & -2.8006\end{array}$

H $5.3019 \quad 3.8123 \quad-5.1894$

H $4.4332 \quad 2.9473 \quad-3.9267$

H $4.3218 \quad 5.8799 \quad-4.1784$

H $2.5449 \quad 6.6040 \quad-2.2502$

H $\quad 0.0730 \quad 5.7223 \quad-3.7995$

H $0.3883 \quad 5.3598 \quad-2.0992$

H $-0.8379 \quad 7.3336-2.3355$

H $6.7557-7.8776-1.5992$

H $5.9850 \quad-9.4853 \quad-1.4453$

H $8.5365 \quad-5.9359-6.9180$

H $7.0439-5.0066-6.7045$

H $8.5995 \quad-4.2667 \quad-6.3182$

H $7.3923-6.6763-4.8021$

H $8.9333 \quad-5.9304 \quad-4.4478$

H $\quad 0.3915 \quad 1.2756 \quad 4.9668$

H $-1.1139 \quad 1.8593 \quad 5.6546$

H $\quad 0.3170 \quad 2.9114 \quad 5.6448$

H $2.5823 \quad 0.9747 \quad 5.5078$

H $3.8019 \quad 0.8204 \quad 6.7825$

H $2.5238 \quad-0.3952 \quad 6.6118$

$\begin{array}{llll}\mathrm{H} & 0.0814 & 0.2872 & 7.2335\end{array}$

H $-0.2146 \quad 1.8693 \quad 7.9327$

H $2.1303 \quad 2.5397 \quad 7.4103$

H $1.5642 \quad 1.6468 \quad 9.6685$

H $3.1995 \quad 1.1513 \quad 9.2033$

H $1.0567 \quad-3.1131 \quad 10.1676$

H $-4.1174 \quad-2.2074 \quad 10.9014$

H $-2.4873-1.5810 \quad 10.6250$

H $-3.8592-0.4710 \quad 10.7329$

H $1.8385 \quad-3.1980 \quad-3.2429$

H $2.5166 \quad-3.7687-1.6977$

H $2.7303 \quad-4.7299 \quad-3.1550$

H $\quad 0.9502-5.6407-1.6211$

H $-0.8120-5.9615-3.2825$

H $-1.2822-3.2613 \quad-3.4884$

H $-1.6198-3.3792-6.3981$

H $-2.8789-1.0201-4.1193$

H $-3.7423 \quad-2.1513 \quad-5.9898$
H $-3.6112-3.6016-4.9689$

H $\quad 0.0787-2.1612-7.2251$

H $0.0499 \quad-0.0861 \quad-8.6011$

$\begin{array}{lllll}\mathrm{H} & -0.4386 & 0.7810 & -7.1172\end{array}$

H 6.8288 -2.9459 -3.4991

$\begin{array}{llll}\mathrm{H} & -6.0231 & 6.1127 & -3.4733\end{array}$

H $-3.3496 \quad 2.4534 \quad-4.3773$

H $\quad 8.7215 \quad 2.8744 \quad-2.1570$

H $5.0542 \quad 1.3257 \quad 4.4346$

H $-0.0085-3.0488-1.8796$

H $\quad 0.5580 \quad-0.6073 \quad-2.0807$

H $-0.2245-1.3129-1.0545$

H $0.4058 \quad 11.9455 \quad-2.0640$

H $-0.3860 \quad 8.7577 \quad 7.7011$

H $0.7959-6.2197 \quad-3.9780$

$\begin{array}{llll}\text { C } & -0.0860 & -0.2071 & 1.1797\end{array}$

$\begin{array}{llll}\text { O } & -0.4653 & -1.2007 & 0.4700\end{array}$

$\begin{array}{llll}\text { O } & -0.3869 & 0.0104 & 2.3618\end{array}$

H $2.1991 \quad-2.1430 \quad 9.2232$

H $\quad 1.8617 \quad-0.0003 \quad 9.1049$

H $\quad \begin{array}{llll}1.2211 & 0.1600 & -7.2780\end{array}$

$\begin{array}{llll}\mathrm{H} & -8.3174 & -0.7388 & -5.8167\end{array}$ H $2.2639 \quad 6.8947 \quad-3.9614$ $\begin{array}{llll}\text { C } & -4.5068 & -5.0712 & -0.5435\end{array}$

H $-9.5134-4.4192-2.2418$

H $-10.5532-3.0396-2.1687$

H $-7.8518-0.8847-1.6603$

H $-9.4423-1.2521 \quad-1.0653$

H $-6.5860 \quad-2.6027-1.8574$

H $-7.6813-5.0043-3.1907$

H $-7.2314-5.0856-1.4771$

H $-5.4505-5.9071-3.0339$

H $-5.4202-4.3444-3.8395$

H $-4.4461-6.1579-0.6875$

H $-5.4156 \quad-4.8584 \quad 0.0235$

H $-3.5065-4.4927-2.3639$

H $-4.5631-3.2761 \quad-1.7044$

H $-3.0061-1.8605-0.0413$ $\mathrm{H}-3.8281 \quad-1.2907 \quad 1.1347$ H $-12.4446-2.8482 \quad 3.1183$ $\mathrm{H}-12.1474-1.4357 \quad 2.0824$ H $-12.5283-2.9950 \quad 1.3539$ $\mathrm{H}-10.3262 \quad-3.9218 \quad 2.2644$ H $-9.9834 \quad-2.3640 \quad 2.9778$ H -12.5359 $-1.8046-1.9646$ H $-11.5945-2.0425-0.7458$ H $-2.4451-4.6283-0.0732$

\section{OC}

N $-3.4276 \quad-4.6703 \quad 0.5507$

C $-4.4682-4.4746-1.6654$

C $-5.5532-4.9642-2.6332$

C $-6.9992-4.6842-2.2146$

N $-7.2600 \quad-3.2495 \quad-2.1485$

C $-8.5044-2.7244-2.0575$

$\mathrm{N}-9.5771 \quad-3.4873 \quad-2.2810$

$\mathrm{N}-8.6711-1.4014-1.8747$

C - $12.0771-2.3214 \quad 2.1286$

C $-10.5840-2.6555 \quad 2.1562$ 
$\begin{array}{llll}\text { C } & -9.8026 & -2.2961 & 0.8868\end{array}$

$\begin{array}{llll}\text { O } & -8.7261 & -2.8700 & 0.6569\end{array}$

$\begin{array}{llll}\mathrm{O}-10.3288 & -1.3759 & 0.1278\end{array}$

O $-12.0430-2.4806-1.5301$

$\begin{array}{llll}\text { O } & -3.2639 & -1.1724 & 0.0870\end{array}$

$\begin{array}{llll}\text { C } & 6.2451 & -8.7145 & -0.1848\end{array}$

$\begin{array}{llll}\text { C } & 5.1123 & -8.0337 & 0.5743\end{array}$

$\begin{array}{llll}\text { O } & 4.0731 & -8.6130 & 0.8790\end{array}$

$\begin{array}{llll}\mathrm{N} & 5.3332 & -6.7270 & 0.9333\end{array}$

C 4.4744 -6.0908 1.9252

$\begin{array}{llll}\text { C } & 3.4600 & -5.1074 & 1.3303\end{array}$

$\begin{array}{llll}\text { O } & 3.8165 & -4.2449 & 0.5095\end{array}$

$\begin{array}{llll}\text { C } & 5.4924 & -5.2932 & 2.7757\end{array}$

$\begin{array}{llll}\text { C } & 6.5708 & -4.8706 & 1.7608\end{array}$

$\begin{array}{llll}\text { C } & 6.6002 & -6.0043 & 0.7197\end{array}$

$\begin{array}{llll}\mathrm{N} & 2.2100 & -5.1283 & 1.8287\end{array}$

$\begin{array}{llll}\text { C } & 1.2359 & -4.1069 & 1.4925\end{array}$

$\begin{array}{llll}\text { C } & -0.0962 & -4.4283 & 2.1607\end{array}$

$\begin{array}{llll}\mathrm{O} & -0.2017 & -5.3490 & 2.9651\end{array}$

$\begin{array}{llll}\mathrm{N} & -1.1261 & -3.6132 & 1.7852\end{array}$

$\begin{array}{llll}\text { C } & -2.3778 & -3.6124 & 2.5293\end{array}$

$\begin{array}{llll}\text { C } & -3.6119 & -4.0850 & 1.7501\end{array}$

$\begin{array}{llll}\text { O } & -4.7291 & -3.9362 & 2.2638\end{array}$

$\begin{array}{llll}\text { C } & -2.6668 & -2.2360 & 3.2188\end{array}$

O $\quad-3.7908-1.5237 \quad 2.6996$

C $8.0929-5.6770 \quad-5.7360$

C $7.5441 \quad-6.0076 \quad-4.3394$

$\begin{array}{llll}\text { C } & 6.9567 & -4.8319 & -3.5318\end{array}$

$\begin{array}{llll}\text { O } & 7.2505 & -3.6516 & -3.9305\end{array}$

$\begin{array}{llll}\text { O } & 6.2497 & -5.0933 & -2.5291\end{array}$

$\begin{array}{llll}\text { C } & 0.1261 & -5.7134 & -3.1732\end{array}$

C $-0.1615-4.8556-4.4129$

$\begin{array}{lllll}\text { O } & 0.2836 & -5.1267 & -5.5251\end{array}$

$\begin{array}{llll}\text { C } & 0.7892 & -4.9928 & -1.9987\end{array}$

$\begin{array}{llll}\text { C } & 2.1045 & -4.3216 & -2.3825\end{array}$

$\begin{array}{llll}\text { O } & -0.1435 & -4.0747 & -1.4075\end{array}$

$\mathrm{N}-1.0073 \quad-3.7843 \quad-4.2384$

$\begin{array}{llll}\text { C } & -1.6420 & -3.1616 & -5.3861\end{array}$

$\begin{array}{llll}\text { C } & -1.0814 & -1.7784 & -5.7997\end{array}$

$\begin{array}{lllll}\text { O } & -1.5241 & -0.7333 & -5.2874\end{array}$

$\begin{array}{llll}\text { C } & -3.1491 & -3.0073 & -5.1352\end{array}$

$\begin{array}{llll}\text { O } & -3.4080 & -2.1528 & -4.0251\end{array}$

$\begin{array}{llll}\mathrm{N} & -0.1878 & -1.7700 & -6.8083\end{array}$

$\begin{array}{llll}\text { C } & 0.2952 & -0.5447 & -7.4576\end{array}$

$\begin{array}{llll}\text { C } & -7.8961 & -0.2999 & -5.2072\end{array}$

$\begin{array}{llll}\mathrm{C} & -6.5202 & -0.6444 & -4.6278\end{array}$

$\begin{array}{llll}\mathrm{N} & -5.9991 & 0.4331 & -3.7746\end{array}$

$\begin{array}{llll}\mathrm{C} & -5.4089 & 0.2832 & -2.5834\end{array}$

N $-5.1054-0.9478-2.1158$

$\begin{array}{llll}\mathrm{N} & -5.1950 & 1.3670 & -1.8203\end{array}$

$\begin{array}{llll}\text { C } & -4.8581 & 5.7539 & -3.7753\end{array}$

$\begin{array}{lllll}C & -4.4825 & 4.3323 & -4.1728\end{array}$

$\begin{array}{lllll}\text { O } & -5.1861 & 3.3747 & -3.8134\end{array}$

$\begin{array}{llll}\text { C } & -4.5629 & 5.9666 & -2.2707\end{array}$

$\begin{array}{llll}\text { C } & -3.0833 & 6.1249 & -1.9248\end{array}$

N $-2.4598 \quad 5.0678-1.4000$

$\begin{array}{lllll}\mathrm{O} & -2.5195 & 7.2297 & -2.1066\end{array}$

$\begin{array}{llll}\mathrm{N} & -3.3304 & 4.1840 & -4.8595\end{array}$

$\begin{array}{lllll}\mathrm{C} & -2.5806 & 2.9241 & -4.9181\end{array}$

C $-1.2550 \quad 3.0732 \quad-4.1439$
C $\quad-0.8442 \quad 1.8220 \quad-3.3766$

$\begin{array}{llll}\text { C } & -1.8737 & 1.4448 & -2.3297\end{array}$

$\begin{array}{llll}\mathrm{N} & -2.1433 & 0.1370 & -2.2159\end{array}$

O $-2.4766 \quad 2.2840-1.6262$

$\begin{array}{lllll}\text { O } & 0.0988 & -1.5486 & -2.2970\end{array}$

C $-1.0226 \quad 8.6781 \quad 6.3526$

$\begin{array}{llll}\mathrm{C} & -0.4001 & 7.2895 & 6.1621\end{array}$

$\begin{array}{llll}\mathrm{N} & -1.0511 & 6.4828 & 5.1212\end{array}$

C $-0.5618 \quad 6.1688 \quad 3.9059$

$\begin{array}{llll}\mathrm{N} & 0.5044 & 6.7829 & 3.3952\end{array}$

N $-1.1689 \quad 5.2216 \quad 3.1799$

C $1.3312 \quad 11.7117-2.0503$

C $\quad 1.6780 \quad 10.2757-1.6696$

$\begin{array}{lllll}\mathrm{N} & 0.5270 & 9.6113 & -1.0546\end{array}$

$\begin{array}{lllll}\text { C } & 0.5981 & 8.4523 & -0.3753\end{array}$

$\begin{array}{lllll}\mathrm{N} & -0.5293 & 7.8218 & -0.0154\end{array}$

$\begin{array}{llll}\mathrm{N} & 1.7768 & 7.9871 & 0.0497\end{array}$

C $2.1575 \quad 5.9260 \quad-3.4602$

C $2.7962 \quad 4.5599-3.6530$

$\begin{array}{llll}\mathrm{O} & 2.2876 & 3.5213 & -3.2219\end{array}$

C $0.6553 \quad 5.8875 \quad-3.2363$

O $\quad 0.2182 \quad 7.2108$-2.9587

N $3.9772 \quad 4.6022 \quad-4.3269$

C $4.9548 \quad 3.5321 \quad-4.2504$

C $6.0874 \quad 3.9778 \quad-3.3088$

$\begin{array}{lllll}\text { O } & 6.3054 & 5.1829 & -3.1597\end{array}$

N $6.7962 \quad 2.9796 \quad-2.7449$

C $7.9000 \quad 3.2542 \quad-1.8295$

$\begin{array}{llll}\text { C } 7.5706 & 2.9562 & -0.3528\end{array}$

$\begin{array}{llll}C & 7.6296 & 1.4498 & -0.0475\end{array}$

$\begin{array}{llll}\text { C } & 6.2303 & 3.5833 & 0.0573\end{array}$

C $7.4920 \quad 1.1223 \quad 1.4409$

C $\quad 1.9454 \quad 1.5748 \quad 8.9474$

C $\quad 1.6995 \quad 2.0908 \quad 7.5226$

$\begin{array}{llll}C & 0.2097 & 1.9806 & 7.1521\end{array}$

$\begin{array}{llll}\text { C } & 2.5976 & 1.3465 & 6.5287\end{array}$

C $-0.1556 \quad 2.5455 \quad 5.7746$

C $\quad 0.9994-2.0870 \quad 9.4048$

$\begin{array}{llll}\text { C } & 0.9093 & -3.1517 & 8.2990\end{array}$

C $1.1178-2.6098 \quad 6.9003$

C $0.1655-1.7585 \quad 6.3206$

C $2.2417 \quad-2.9585 \quad 6.1420$

C $0.3207-1.2806 \quad 5.0211$

C $2.3997-2.4920 \quad 4.8337$

C $1.4356-1.6578 \quad 4.2697$

C $-3.7821-0.7896 \quad 10.3943$

$\begin{array}{llll}\text { C } & -3.9376 & -0.9431 & 8.8784\end{array}$

$\begin{array}{llll}\text { C } & -3.0688 & 0.0084 & 8.0362\end{array}$

$\begin{array}{llll}\mathrm{C} & -3.2112 & -0.3133 & 6.5436\end{array}$

$\begin{array}{llll}\text { C } & -3.3961 & 1.4834 & 8.3001\end{array}$

C $\quad 0.4742 \quad 2.2996 \quad 0.7227$

C $\quad 0.9550 \quad 4.7552 \quad 0.6826$

$\begin{array}{lllll}\text { O } & 1.0842 & 0.6977 & -0.9699\end{array}$

$\begin{array}{llll}\mathrm{O} & -0.2692 & 5.0909 & 0.5111\end{array}$

O $1.7829 \quad 5.4986 \quad 1.2930$

C $7.2952-0.4968-4.0280$

C $5.1889-1.6233 \quad 1.7250$

C $3.0563-1.3587-0.2701$

C $4.9664-2.1450-1.6823$

C $4.1624-1.1214-1.2607$ $\begin{array}{llll}\text { C } & 4.3389 & 0.1861 & -1.8724\end{array}$

N $3.5127 \quad 1.1645-1.6030$

N $5.4081 \quad 0.3626-2.7405$

$\begin{array}{llll}\text { C } & 6.1387 & -0.6764 & -3.0893\end{array}$

N $5.9305 \quad-1.9231 \quad-2.6173$

$\begin{array}{llll}\text { S } & 2.6319 & 1.3380 & 2.6249\end{array}$

$\begin{array}{llll}\text { C } & 2.2409 & 0.5380 & 1.1609\end{array}$

N $3.1203 \quad-0.4388 \quad 0.9110$

$\begin{array}{llll}\text { C } & 4.1459 & -0.5697 & 1.8617\end{array}$

$\begin{array}{llll}\text { C } & 4.0268 & 0.3298 & 2.8774\end{array}$

C $4.8695 \quad 0.4568 \quad 4.1063$

$\begin{array}{lllll}\mathrm{C} & 0.9579 & 0.8669 & 0.4043\end{array}$

$\begin{array}{llll}\text { C } & 1.4026 & 3.3999 & 0.1787\end{array}$

H $3.70292 .0063-2.1392$

H $-0.5113 \quad 2.4032 \quad 0.2623$

$\begin{array}{llll}\mathrm{H} & 0.3225 & 2.3994 & 1.8019\end{array}$

H $8.1904-0.2355 \quad-3.4514$

H $7.0899 \quad 0.3189-4.7223$

H $7.5042-1.4274 \quad-4.5594$

H $4.7406 \quad-2.5913 \quad 1.4961$

H $5.7499 \quad-1.7105 \quad 2.6551$

H $5.8879 \quad-1.3884 \quad 0.9191$

H $4.4475 \quad-0.1409 \quad 4.9204$

H $1.3785 \quad 3.3954-0.9169$

H $4.8990-3.1709-1.3292$

H $2.0773-1.2063 \quad-0.7098$

H $5.8861 \quad 0.1139 \quad 3.9086$

$\begin{array}{llll}\mathrm{H} & 2.4416 & 3.2499 & 0.4767\end{array}$

H $3.1138 \quad-2.3757 \quad 0.1179$

H $-0.4186 \quad-0.6261 \quad 4.5752$

H $\quad \begin{array}{lll}1.5283 & -1.3032 & 3.2515\end{array}$

H $-0.7154-1.4824 \quad 6.8912$

H $2.9883 \quad-3.6206 \quad 6.5722$

H $3.2655 \quad-2.7971 \quad 4.2517$

H $-0.0762-3.6312 \quad 8.3482$

H 1.6438 -3.9403 8.4936

H $\quad 0.2587 \quad-1.2957 \quad 9.2527$

H $-3.6913-1.9747 \quad 8.5962$

H $-4.9904-0.7972 \quad 8.5994$

H $-4.4518 \quad 1.6876 \quad 8.0836$

H $-3.2065 \quad 1.76719 .3392$

H $-2.7918 \quad 2.1375 \quad 7.6622$

H $-2.0193-0.1576 \quad 8.3254$

H $-4.2347 \quad-0.1281 \quad 6.1989$

$\begin{array}{llll}\mathrm{H} & -2.5395 & 0.2988 & 5.9318\end{array}$

H $-2.9833-1.3640 \quad 6.3391$

H $-1.7335 \quad 4.5196 \quad 3.6325$

H $-0.9306 \quad 5.1084 \quad 2.1826$

$\begin{array}{llll}\mathrm{H} & 0.8497 & 7.6329 & 3.8086\end{array}$

H $1.0069 \quad 6.3604 \quad 2.5866$

H $-1.9594 \quad 6.1011 \quad 5.3434$

$\begin{array}{llll}\mathrm{H} & 0.6620 & 7.3597 & 5.9144\end{array}$

H $-0.4565 \quad 6.7192 \quad 7.0944$

$\begin{array}{llll}\mathrm{H} & -0.9511 & 9.2760 & 5.4394\end{array}$

H $-2.0786 \quad 8.5999 \quad 6.6263$

H $-0.4213 \quad 6.8520 \quad 0.3220$

H $-1.3346 \quad 7.9250-0.6329$

H $-0.3722 \quad 9.8132-1.4701$

$\begin{array}{llll}\text { H } & 2.6299 & 8.4097 & -0.2735\end{array}$

$\begin{array}{llll}\mathrm{H} & 1.8320 & 7.0645 & 0.5077\end{array}$ 
H $2.0119 \quad 9.7024 \quad-2.5435$

H $2.4934 \quad 10.2976 \quad-0.9395$

H $2.2004 \quad 12.1960-2.5010$

H $1.0284 \quad 12.2867 \quad-1.1715$

H $7.1166-8.8508 \quad 0.4641$

H $7.4452-6.6762 \quad 0.9011$

H $6.6608-5.6298-0.3076$

H $7.5453 \quad-4.7248 \quad 2.2342$

H $6.2926-3.9412 \quad 1.2679$

H $5.9133 \quad-5.9666 \quad 3.5286$

H $5.0371 \quad-4.4484 \quad 3.3025$

H $3.9533 \quad-6.8695 \quad 2.4863$

$\begin{array}{llll}\mathrm{H} & 1.8921 & -5.8366 & 2.4817\end{array}$

H $1.0803 \quad-4.0255 \quad 0.4153$

H $1.5703 \quad-3.1259 \quad 1.8517$

H $-0.9054-2.8030 \quad 1.2032$

H $-2.2423-4.3663 \quad 3.3115$

H $-1.8042-1.5858 \quad 3.0774$

H $-2.8089-2.4034 \quad 4.2911$

H $-4.5218-2.1783 \quad 2.7023$

H $-4.7372-1.5845-2.8289$

$\begin{array}{llll}\mathrm{H} & -4.5564 & -0.9874 & -1.2469\end{array}$

H $-4.3663 \quad 1.3698-1.2303$

H $-5.3487 \quad 2.2612-2.2820$

H $-6.0276 \quad 1.3858-4.1271$

H $-6.5975-1.5461-4.0184$

H $-5.7962-0.8472 \quad-5.4254$

$\begin{array}{llll}\mathrm{H} & -7.8656 & 0.6264 & -5.7906\end{array}$

H $-8.6276-0.1973-4.4024$

H $-1.4828-0.5335-2.6065$

H $-2.6121-0.1505-1.3595$

$\begin{array}{llll}\mathrm{H} & -0.6923 & 0.9788 & -4.0538\end{array}$

$\begin{array}{lllll}\mathrm{H} & 0.0996 & 2.0055 & -2.8527\end{array}$

H $-0.4436 \quad 3.3606-4.8187$

$\begin{array}{llll}\mathrm{H} & -1.3773 & 3.8815 & -3.4218\end{array}$

H $-2.4136 \quad 2.6341-5.9595$

H $-2.8151 \quad 5.0272-5.0715$

$\begin{array}{llll}\mathrm{H} & -4.3271 & 6.5047 & -4.3685\end{array}$

H $\quad-5.0053 \quad 5.1434-1.7036$

H $-5.0424 \quad 6.8953-1.9518$

H $-1.5148 \quad 5.1442-1.0264$

H $-2.8147 \quad 4.1123-1.4307$

H $7.6664 \quad 0.0589 \quad 1.6293$

H $8.2193 \quad 1.6864 \quad 2.0355$

$\begin{array}{llll}\mathrm{H} & 6.4934 & 1.3688 & 1.8121\end{array}$

$\begin{array}{llll}\text { H } & 6.8444 & 0.9330 & -0.6129\end{array}$

H $8.5860 \quad 1.0549 \quad-0.4136$

H $5.3926 \quad 3.0235 \quad-0.3729$

$\begin{array}{llll}\mathrm{H} & 6.1067 & 3.5822 & 1.1440\end{array}$

$\begin{array}{llll}\mathrm{H} & 6.1586 & 4.6173 & -0.2927\end{array}$

$\begin{array}{llll}\mathrm{H} & 8.3704 & 3.4367 & 0.2298\end{array}$

H $8.1368 \quad 4.3123 \quad-1.9519$

H $6.4012 \quad 2.0348 \quad-2.8011$

H $5.3845 \quad 3.3486 \quad-5.2421$

H $4.4718 \quad 2.6102-3.9299$

H $4.4231 \quad 5.5122-4.3809$

H $2.6250 \quad 6.3807 \quad-2.5755$

H $0.1624 \quad 5.4897 \quad-4.1334$

H $\quad 0.4300 \quad 5.2001 \quad-2.4109$

H $-0.7386 \quad 7.1798-2.7629$
H $\quad 6.5548 \quad-8.1179-1.0488$

H $\quad 5.8901 \quad-9.6903-0.5137$

H $8.5271-6.5653 \quad-6.2063$

H $7.3000 \quad-5.2973 \quad-6.3881$

H $8.8596 \quad-4.9008 \quad-5.6721$

H $6.7672-6.7782-4.3932$

H $8.3409 \quad-6.4405 \quad-3.7187$

$\begin{array}{llll}\mathrm{H} & 0.2827 & 1.9587 & 4.9620\end{array}$

$\mathrm{H}-1.2422 \quad 2.5322 \quad 5.6280$

H $\quad 0.1909 \quad 3.5824 \quad 5.6677$

$\begin{array}{llll}\text { H } & 2.4997 & 1.7508 & 5.5169\end{array}$

H $3.6492 \quad 1.4304 \quad 6.8216$

$\begin{array}{lllll}\mathrm{H} & 2.3379 & 0.2837 & 6.4869\end{array}$

H $-0.0856 \quad 0.9260 \quad 7.1935$

H $-0.3797 \quad 2.4946 \quad 7.9219$

H $1.9716 \quad 3.1578 \quad 7.4932$

H $\quad 1.3328 \quad 2.1122 \quad 9.6793$

H $2.9949 \quad 1.6925 \quad 9.2359$

H $\quad 0.8169-2.5314 \quad 10.3878$

H $-4.3661-1.5467 \quad 10.9258$

H $-2.7345-0.9056 \quad 10.6961$

H $-4.1215 \quad 0.1904 \quad 10.7432$

H $1.9411-3.5350 \quad-3.1268$

H $2.5780 \quad-3.8929-1.5003$

H $2.8088-5.0452 \quad-2.8015$

H $\quad 0.9945 \quad-5.7522-1.2343$

H $-0.8217-6.1407-2.8255$

$\mathrm{H}-1.3172-3.5382-3.3075$

H $-1.4915-3.8526-6.2206$

H $-2.8958-1.3378-4.2207$

H $-3.6359-2.6293-6.0456$

H $-3.5712-3.9868-4.8995$

H $\quad 0.1926-2.6646 \quad-7.0921$

H $\quad 0.3386 \quad-0.7013 \quad-8.5372$

$\begin{array}{llll}\mathrm{H} & -0.4080 & 0.2545 & -7.2250\end{array}$

H $\quad 6.4778 \quad-2.7090 \quad-3.0477$

H $-5.9302 \quad 5.8752 \quad-3.9434$

H $-3.2258 \quad 2.1683 \quad-4.4687$

H $\quad 8.7751 \quad 2.6715 \quad-2.1412$

H $4.9274 \quad 1.4932 \quad 4.4454$

H $\quad 0.0651 \quad-3.1774-1.7444$

H $\quad 0.8339-1.0570 \quad-2.6823$

H $\quad 0.0143-1.1823 \quad-1.3717$

H $\quad 0.5185 \quad 11.7409 \quad-2.7837$

$\begin{array}{llll}\mathrm{H} & -0.5034 & 9.2070 & 7.1562\end{array}$

H $\quad 0.7629 \quad-6.5307 \quad-3.5183$

C $-0.1125-0.1508 \quad 1.0097$

$\begin{array}{llll}\text { O } & -0.4671 & -1.1082 & 0.2395\end{array}$

$\begin{array}{llll}\text { O } & -0.4781 & 0.0646 & 2.1690\end{array}$

H $1.9908 \quad-1.6251 \quad 9.4199$

H $\quad 1.6955 \quad 0.5140 \quad 9.0182$

H $1.2884-0.2629-7.0938$

H $-8.2201-1.1052-5.8716$

$\begin{array}{llll}\text { H } & 2.3891 & 6.5819 & -4.3058\end{array}$

$\begin{array}{llll}\text { C } & -4.5582 & -5.0975 & -0.2718\end{array}$

H $-9.5028-4.4698-2.0679$

$\mathrm{H}-10.5180-3.0735-2.1298$

H $-7.8021-0.9340-1.6453$

H $-9.4170-1.2391-1.0932$

H $-6.5570-2.6937-1.6780$
H $-7.6657-5.1143 \quad-2.9682$

H $-7.2304-5.1560-1.2502$

H $-5.4534-6.0486-2.7605$

H $-5.3887-4.5147 \quad-3.6189$

H $-4.5750 \quad-6.1930 \quad-0.3434$

H $-5.4568 \quad-4.7827 \quad 0.2630$

H $-3.4894-4.7070-2.1034$

H $-4.4790 \quad-3.3875-1.5526$

H $-2.2920 \quad-1.29390 .1440$

H $-3.5470-1.1481 \quad 1.0361$

H $-12.5603 \quad-2.6088 \quad 3.0678$ $\mathrm{H}-12.2280-1.2503 \quad 1.9726$

H - $12.5835 \quad-2.8452 \quad 1.3116$

H - $10.4132 \quad-3.7195 \quad 2.3476$ $\mathrm{H}-10.0947 \quad-2.1232 \quad 2.9834$ H -12.4930 - $1.8046-2.0510$ H -11.5867 $-1.9862-0.7958$ H $-2.4854 \quad-4.7044 \quad 0.1775$ H $2.0620 \quad 0.9524 \quad-1.2508$

\section{TS2}

$\begin{array}{llll}\mathrm{N} & -3.3458 & -4.6490 & 0.0928\end{array}$

C $-4.4865-4.4726-2.0742$

C $-5.6172-4.9428-2.9973$

$\begin{array}{llll}\text { C } & -7.0387 & -4.6922 & -2.4844\end{array}$

N $-7.2934 \quad-3.2668-2.2833$

C $-8.5352-2.7712-2.0393$

$\mathrm{N}-9.6148 \quad-3.5210-2.2650$

N $-8.6948-1.4873 \quad-1.6810$

C - $11.9124 \quad-2.8360 \quad 2.3691$

C - $10.4186-3.1665 \quad 2.2868$

C $-9.6768-2.6582 \quad 1.0424$

$\begin{array}{lllll}\text { O } & -8.5872 & -3.1684 & 0.7369\end{array}$

O $-10.2460-1.6864 \quad 0.3842$

O $-12.0388-2.6629-1.2641$

$\begin{array}{llll}\text { O } & -3.3631 & -1.5366 & 0.0060\end{array}$

C 6.3695 -8.5182 -1.5568

C $5.2043-7.9586-0.7465$

$\begin{array}{lllll}\text { O } & 4.1193 & -8.5296 & -0.6680\end{array}$

N $5.4381-6.7857 \quad-0.0735$

$\begin{array}{llll}\text { C } & 4.5117 & -6.3522 & 0.9666\end{array}$

$\begin{array}{llll}\text { C } & 3.4971 & -5.2934 & 0.5163\end{array}$

$\begin{array}{llll}\text { O } & 3.8115 & -4.3302 & -0.1919\end{array}$

$\begin{array}{llll}\text { C } & 5.4559 & -5.7002 & 2.0047\end{array}$

$\begin{array}{llll}\text { C } & 6.5463 & -5.0684 & 1.1252\end{array}$

C $6.7277-6.0764-0.0214$

$\begin{array}{llll}\text { N } & 2.2671 & -5.3991 & 1.0681\end{array}$

C $1.3060-4.3240 \quad 0.9856$

$\begin{array}{llll}C & 0.0002 & -4.7566 & 1.6296\end{array}$

$\begin{array}{llll}\text { O } & -0.1086 & -5.8104 & 2.2451\end{array}$

N $-1.0257 \quad-3.8667 \quad 1.4573$

$\begin{array}{llll}\text { C } & -2.2458 & -4.0098 & 2.2305\end{array}$

$\begin{array}{llll}\text { C } & -3.4870 & -4.4199 & 1.4297\end{array}$

$\begin{array}{llll}\text { O } & -4.5768 & -4.4632 & 2.0022\end{array}$

C $-2.5839-2.7505 \quad 3.0914$

$\begin{array}{llll}\text { O } & -3.6955 & -1.9870 & 2.6049\end{array}$

$\begin{array}{llll}\text { C } & 7.8961 & -4.7979 & -6.7803\end{array}$

C $8.0324-5.2235 \quad-5.3108$

C $7.2119-4.4477 \quad-4.2879$ 
O $7.0985-3.1486-4.5710$

$\begin{array}{lllll}\text { O } & 6.7454 & -4.9613 & -3.2834\end{array}$

$\begin{array}{llll}\text { C } & 0.0661 & -5.3095 & -3.8703\end{array}$

C $-0.3487-4.3637 \quad-5.0111$

$\begin{array}{lllll}\text { O } & -0.0142 & -4.5469 & -6.1773\end{array}$

$\begin{array}{llll}\text { C } & 0.4412 & -4.6176 & -2.5519\end{array}$

C $1.5539-3.5880-2.7416$

O $-0.7554-4.0320-1.9882$

N $-1.1653-3.3142-4.6577$

C $-1.8661-2.5497-5.6720$

C $-1.3014-1.1297-5.9221$

$\begin{array}{llll}\text { O } & -1.6257 & -0.1877 & -5.1753\end{array}$

$\begin{array}{llll}\text { C } & -3.3465 & -2.4050 & -5.2833\end{array}$

O $-3.4949-1.7383-4.0305$

$\begin{array}{llll}\mathrm{N} & -0.5424 & -0.9628 & -7.0234\end{array}$

$\begin{array}{llll}\text { C } & -0.0539 & 0.3387 & -7.5012\end{array}$

$\begin{array}{llll}\text { C } & -8.1277 & 0.1392 & -4.8563\end{array}$

$\begin{array}{llll}\text { C } & -6.6781 & -0.1911 & -4.5032\end{array}$

$\begin{array}{llll}\mathrm{N} & -6.1138 & 0.8025 & -3.5829\end{array}$

$\begin{array}{llll}\mathrm{C} & -5.5582 & 0.5510 & -2.3966\end{array}$

$\mathrm{N}-5.2446-0.7191 \quad-2.0452$

$\begin{array}{llll}\mathrm{N} & -5.3747 & 1.5604 & -1.5296\end{array}$

$\begin{array}{llll}\text { C } & -5.1158 & 6.0398 & -2.8467\end{array}$

$\begin{array}{llll}\text { C } & -4.7731 & 4.7142 & -3.5245\end{array}$

$\begin{array}{lllll}\text { O } & -5.5056 & 3.7234 & -3.3645\end{array}$

$\begin{array}{llll}\text { C } & -4.7307 & 5.9107 & -1.3544\end{array}$

$\begin{array}{llll}\mathrm{C} & -3.2478 & 6.1368 & -1.0926\end{array}$

$\begin{array}{llll}\mathrm{N} & -2.5390 & 5.1282 & -0.5732\end{array}$

$\begin{array}{llll}\text { O } & -2.7688 & 7.2707 & -1.3215\end{array}$

$\begin{array}{llll}\mathrm{N} & -3.6031 & 4.6602 & -4.1940\end{array}$

$\begin{array}{llll}\text { C } & -2.8541 & 3.4163 & -4.4289\end{array}$

$\begin{array}{llll}C & -1.5183 & 3.4878 & -3.6523\end{array}$

$\begin{array}{llll}C & -1.0444 & 2.1715 & -3.0464\end{array}$

$\begin{array}{llll}\text { C } & -2.0132 & 1.6177 & -2.0257\end{array}$

$\begin{array}{llll}\mathrm{N} & -2.1490 & 0.2805 & -2.0274\end{array}$

$\begin{array}{llll}\text { O } & -2.7012 & 2.3333 & -1.2664\end{array}$

$\begin{array}{llll}\text { O } & -0.5389 & -1.6928 & -0.6718\end{array}$

$\begin{array}{llll}\text { C } & -0.8328 & 7.8070 & 7.3696\end{array}$

$\begin{array}{llll}\text { C } & 0.1491 & 6.8338 & 6.7037\end{array}$

$\begin{array}{llll}\mathrm{N} & -0.4855 & 5.8263 & 5.8361\end{array}$

$\begin{array}{llll}\mathrm{C} & -0.2976 & 5.6840 & 4.5100\end{array}$

$\begin{array}{llll}\mathrm{N} & 0.2043 & 6.6802 & 3.7676\end{array}$

$\begin{array}{llll}\mathrm{N} & -0.6168 & 4.5454 & 3.8876\end{array}$

C $1.0593 \quad 11.8763-0.7060$

C $0.3715 \quad 10.7146-1.4191$

$\begin{array}{lllll}\mathrm{N} & -0.4417 & 9.8920 & -0.5219\end{array}$

$\begin{array}{llll}\text { C } & 0.0147 & 8.8191 & 0.1440\end{array}$

$\begin{array}{llll}\mathrm{N} & -0.8823 & 7.9774 & 0.6904\end{array}$

$\begin{array}{llll}\mathrm{N} & 1.3168 & 8.6185 & 0.3573\end{array}$

$\begin{array}{lllll}C & 1.9036 & 6.3204 & -2.8424\end{array}$

C $2.5337 \quad 5.0265 \quad-3.3255$

$\begin{array}{lllll}\text { O } & 1.9801 & 3.9263 & -3.1827\end{array}$

C $0.3849 \quad 6.2718 \quad-2.8143$

$\begin{array}{lllll}\text { O } & -0.0987 & 7.5274 & -2.3604\end{array}$

N $3.7480 \quad 5.1883 \quad-3.8983$

C $4.7116 \quad 4.1171 \quad-4.0641$

C $5.9309 \quad 4.4719 \quad-3.2017$

$\begin{array}{lllll}\text { O } & 6.2058 & 5.6555 & -2.9971\end{array}$

$\begin{array}{llll}\mathrm{N} & 6.6374 & 3.4203 & -2.7399\end{array}$

C $7.7592 \quad 3.5935-1.8176$
C $7.5275 \quad 2.8643 \quad-0.4803$

C $7.6230 \quad 1.3367 \quad-0.6491$

$\begin{array}{llll}C & 6.2034 & 3.2931 & 0.1694\end{array}$

$\begin{array}{llll}\text { C } & 7.6197 & 0.5614 & 0.6695\end{array}$

$\begin{array}{llll}\text { C } & 2.3667 & 0.5077 & 8.9443\end{array}$

$\begin{array}{llll}\text { C } & 1.9289 & 1.1642 & 7.6282\end{array}$

$\begin{array}{llll}\text { C } & 0.4815 & 1.6691 & 7.7541\end{array}$

$\begin{array}{llll}\text { C } & 2.1002 & 0.2002 & 6.4491\end{array}$

$\begin{array}{llll}\text { C } & -0.0169 & 2.4493 & 6.5357\end{array}$

C $1.5002 \quad-3.2021 \quad 8.9993$

C $1.8297 \quad-4.2813 \quad 7.9510$

C $2.0072 \quad-3.6959 \quad 6.5690$

C $0.8983 \quad-3.4998 \quad 5.7384$

C $3.2629 \quad-3.2793 \quad 6.1088$

C $1.0288-2.8655 \quad 4.5044$

C $3.4015-2.6545 \quad 4.8685$

$\begin{array}{llll}\text { C } & 2.2805 & -2.4288 & 4.0686\end{array}$

C $-3.2461 \quad-2.1334 \quad 10.3613$

$\begin{array}{llll}\text { C } & -3.0782 & -2.2468 & 8.8395\end{array}$

C $-2.3506-1.0623 \quad 8.1776$

$\begin{array}{llll}\text { C } & -2.1340 & -1.3123 & 6.6801\end{array}$

$\begin{array}{llll}\text { C } & -3.0970 & 0.2614 & 8.3901\end{array}$

$\begin{array}{llll}C & 0.6233 & 2.5994 & 0.9951\end{array}$

C $\quad \begin{array}{llll}1.1035 & 5.1154 & 0.6581\end{array}$

O $0.6108 \quad 0.6231 \quad-0.4199$

$\begin{array}{llll}\text { O } & -0.0261 & 5.3712 & 1.2497\end{array}$

$\begin{array}{lllll}\text { O } & 1.9552 & 5.9974 & 0.4137\end{array}$

$\begin{array}{llll}\text { C } & 6.7423 & 0.1788 & -4.3888\end{array}$

$\begin{array}{llll}\text { C } & 5.0294 & -1.7642 & 1.6427\end{array}$

$\begin{array}{llll}\text { C } & 2.9543 & -1.1377 & -0.3064\end{array}$

C $4.8186-1.7160-1.9177$

$\begin{array}{llll}\text { C } & 3.9069 & -0.7972 & -1.4317\end{array}$

$\begin{array}{llll}\text { C } & 3.8560 & 0.4520 & -2.1250\end{array}$

$\begin{array}{llll}\mathrm{N} & 2.8770 & 1.3414 & -1.9039\end{array}$

$\begin{array}{llll}\mathrm{N} & 4.8132 & 0.7652 & -3.0375\end{array}$

$\begin{array}{llll}\text { C } & 5.6777 & -0.1932 & -3.3930\end{array}$

N $5.6911 \quad-1.4456 \quad-2.9183$

$\begin{array}{llll}\text { S } & 3.1408 & 1.7098 & 2.5154\end{array}$

$\begin{array}{llll}\text { C } & 2.3412 & 0.7910 & 1.2323\end{array}$

$\begin{array}{llll}\mathrm{N} & 3.1185 & -0.3133 & 0.9145\end{array}$

C $4.2040 \quad-0.5274 \quad 1.7892$

$\begin{array}{llll}\text { C } & 4.3589 & 0.4410 & 2.7190\end{array}$

C $\quad 5.3435 \quad 0.5575 \quad 3.8358$

$\begin{array}{llll}C & 1.1027 & 1.1863 & 0.6795\end{array}$

C $\quad \begin{array}{llll}1.3885 & 3.6863 & 0.2218\end{array}$

H $2.8067 \quad 2.1650 \quad-2.4942$

H $2.0163 \quad 1.0544 \quad-1.4119$

$\begin{array}{llll}\mathrm{H} & -0.4416 & 2.6382 & 0.7476\end{array}$

H $\quad 0.7168 \quad 2.7848 \quad 2.0685$

H $7.6470 \quad 0.5006 \quad-3.8588$

H $6.4080 \quad 1.0040 \quad-5.0196$

H $7.0139-0.6795 \quad-5.0050$

H $4.4195 \quad-2.6663 \quad 1.7314$

H $5.8032 \quad-1.7962 \quad 2.4092$

H $5.5223 \quad-1.80290 .6690$

H $4.8455 \quad 0.5190 \quad 4.8112$

H $1.1711 \quad 3.6288 \quad-0.8518$

H $4.8504-2.7253-1.5130$

H $1.9253 \quad-0.9997 \quad-0.6206$

H $6.0757 \quad-0.2513 \quad 3.7984$
H $\quad 2.4691 \quad 3.5401 \quad 0.3017$

H $3.0985-2.1839 \quad-0.0385$

$\begin{array}{llll}\text { H } & 0.1625 & -2.6875 & 3.8815\end{array}$

H $2.3680-1.8987 \quad 3.1279$

H $-0.0779-3.8437 \quad 6.0711$

H $4.1369-3.4360 \quad 6.7360$

H $4.3805 \quad-2.3243 \quad 4.5355$

H $\quad 1.0267 \quad-5.0269 \quad 7.9330$

H $2.7420 \quad-4.8091 \quad 8.2508$

H $0.5899-2.6569 \quad 8.7225$

H $-2.5254 \quad-3.1660 \quad 8.6059$

$\begin{array}{llll}\mathrm{H} & -4.0659 & -2.3594 & 8.3705\end{array}$

$\begin{array}{llll}\mathrm{H} & -4.1123 & 0.1984 & 7.9803\end{array}$

$\begin{array}{llll}\mathrm{H} & -3.1781 & 0.5225 & 9.4488\end{array}$

H $-2.5855 \quad 1.0862 \quad 7.8824$

H $-1.3609-0.9799 \quad 8.6525$

$\begin{array}{llll}\mathrm{H} & -3.0947 & -1.3598 & 6.1523\end{array}$

H $-1.5433-0.5154 \quad 6.2153$

H $-1.6018 \quad-2.2498 \quad 6.5039$

$\mathrm{H}-0.6571 \quad 3.6668 \quad 4.3831$

$\begin{array}{llll}\mathrm{H} & -0.5072 & 4.5239 & 2.8665\end{array}$

$\begin{array}{llll}\mathrm{H} & 0.1315 & 7.6369 & 4.0713\end{array}$

$\begin{array}{llll}\mathrm{H} & 0.3212 & 6.4777 & 2.7702\end{array}$

H $-0.9039 \quad 5.0319 \quad 6.3010$

$\begin{array}{llll}\mathrm{H} & 0.8893 & 7.3703 & 6.1054\end{array}$

$\begin{array}{llll}\mathrm{H} & 0.7122 & 6.2901 & 7.4694\end{array}$

H $-1.3854 \quad 8.3885 \quad 6.6259$

H $-1.5568 \quad 7.2726 \quad 7.9908$

H $-0.5144 \quad 7.0526 \quad 0.9696$

H $-1.7458 \quad 7.8932 \quad 0.1535$

H $-1.4450 \quad 9.9572 \quad-0.6069$

$\begin{array}{llll}\mathrm{H} & 1.9716 & 9.2397 & -0.0910\end{array}$

H $1.6184 \quad 7.6219 \quad 0.4827$

H $-0.3008 \quad 11.0934-2.1925$

H $1.0833 \quad 10.0595 \quad-1.9319$

H $1.644612 .4641 \quad-1.4188$

H $1.7362 \quad 11.5276 \quad 0.0803$

H $7.1991 \quad-8.7973-0.8986$

H $7.5387 \quad-6.7808 \quad 0.2013$

H $6.9389 \quad-5.5897 \quad-0.9768$

H $7.4780 \quad-4.8888 \quad 1.6677$

$\begin{array}{llll}\text { H } & 6.1905 & -4.1204 & 0.7206\end{array}$

H $\quad \begin{array}{llll}5.8762 & -6.4877 & 2.6386\end{array}$

H $4.9436-4.9833 \quad 2.6530$

H $3.9822 \quad-7.2262 \quad 1.3530$

H $1.9927 \quad-6.1933 \quad 1.6338$

H $\quad 1.1221-4.0293 \quad-0.0469$

H $1.6697 \quad-3.4311 \quad 1.5074$

H $-0.7820 \quad-2.9641 \quad 1.0549$

H $-2.0628-4.85292 .9050$

H $-1.7292-2.0745 \quad 3.0839$

H $-2.7867 \quad-3.0556 \quad 4.1214$

H $-4.4566 \quad-2.5990 \quad 2.6244$

H $-4.8193-1.2662-2.8024$

H $-4.7604-0.8455-1.1501$

H $-4.4946 \quad 1.5655-1.0111$

H $-5.60092 .4817-1.9010$

H $-6.1609 \quad 1.7862-3.8396$

H $-6.6297-1.1678-4.0200$

H $\quad-6.0501 \quad-0.2348 \quad-5.3999$ 
$\begin{array}{llll}\mathrm{H} & -8.2146 & 1.1399 & -5.2918\end{array}$

$\begin{array}{llll}\mathrm{H} & -8.7570 & 0.0778 & -3.9651\end{array}$

H $-1.4083-0.2781-2.4314$

H $-2.5826-0.1473-1.2184$

H $-0.8676 \quad 1.4218 \quad-3.8202$

$\begin{array}{llll}\mathrm{H} & -0.0934 & 2.3322 & -2.5280\end{array}$

$\begin{array}{llll}\mathrm{H} & -0.7214 & 3.8783 & -4.2899\end{array}$

H $-1.6493 \quad 4.2006-2.8372$

$\begin{array}{llll}\mathrm{H} & -2.6950 & 3.2669 & -5.5005\end{array}$

H $-3.0691 \quad 5.5175 \quad-4.2444$

$\begin{array}{llll}\mathrm{H}-4.5963 & 6.8930 & -3.2910\end{array}$

$\begin{array}{llll}\mathrm{H} & -5.0673 & 4.9471 & -0.9643\end{array}$

H $-5.2443 \quad 6.6937-0.7884$

H $-1.5829 \quad 5.2763-0.2532$

H $-2.8315 \quad 4.1533-0.6475$

$\begin{array}{llll}\text { H } & 7.7624 & -0.5088 & 0.4913\end{array}$

H $8.4262 \quad 0.9006 \quad 1.3296$

$\begin{array}{llll}\text { H } & 6.6727 & 0.6850 & 1.1998\end{array}$

H $\quad 6.7845 \quad 0.9862 \quad-1.2621$

H $\quad 8.5391 \quad 1.0990 \quad-1.2057$

H $5.3445 \quad 2.9013 \quad-0.3862$

H $6.1208 \quad 2.9210 \quad 1.1935$

H $6.1119 \quad 4.3834 \quad 0.1984$

$\begin{array}{llll}\text { H } & 8.3538 & 3.1761 & 0.1752\end{array}$

H $7.8611 \quad 4.6689-1.6637$

H $6.2127 \quad 2.5029 \quad-2.8486$

H $5.0328 \quad 4.0504 \quad-5.1111$

$\begin{array}{lllll}\mathrm{H} & 4.2758 & 3.1568 & -3.7924\end{array}$

H $4.2066 \quad 6.0859-3.7726$

$\begin{array}{llll}\text { H } & 2.2611 & 6.4802 & -1.8183\end{array}$

H $0.0002 \quad 6.0431 \quad-3.8202$

$\begin{array}{lllll}\mathrm{H} & 0.0686 & 5.4547 & -2.1560\end{array}$

H $-1.0375 \quad 7.4249-2.1208$

H $6.7406 \quad-7.7744-2.2697$

H $6.0165 \quad-9.4015 \quad-2.0871$

H $8.5452 \quad-5.4104 \quad-7.4119$

H $6.8673-4.9193 \quad-7.1324$

$\begin{array}{llll}\text { H } & 8.1733 & -3.7492 & -6.9097\end{array}$

H $7.7756-6.2773-5.1762$

H $9.0803 \quad-5.1186-4.9973$

H $-0.0633 \quad 1.8176 \quad 5.6405$

$\begin{array}{llll}\mathrm{H} & -1.0262 & 2.8431 & 6.7227\end{array}$

$\begin{array}{llll}\mathrm{H} & 0.6607 & 3.2858 & 6.3214\end{array}$

H $1.8686 \quad 0.6636 \quad 5.4862$

$\begin{array}{llll}\text { H } & 3.1273 & -0.1716 & 6.3987\end{array}$

H $1.4515 \quad-0.6752 \quad 6.5573$

$\begin{array}{llll}\mathrm{H} & -0.1756 & 0.8109 & 7.9247\end{array}$

$\begin{array}{llll}\mathrm{H} & 0.3975 & 2.3009 & 8.6481\end{array}$

$\begin{array}{llll}\text { H } & 2.5737 & 2.0399 & 7.4527\end{array}$

H $2.3322 \quad 1.2179 \quad 9.7775$

H $3.3884 \quad 0.1199 \quad 8.8736$

H $1.3431 \quad-3.6468 \quad 9.9879$

H $-3.6987 \quad-3.0409 \quad 10.7710$

H $-2.2749-1.9953 \quad 10.8518$

H $-3.8835-1.2916 \quad 10.6398$

H 1.2268 -2.7734 -3.3941

H $1.8714 \quad-3.1655-1.7891$

H $2.4329 \quad-4.0634 \quad-3.1846$

H $0.7989-5.3971-1.8656$

H $-0.7681 \quad-5.9975 \quad-3.6872$
H $-1.4234-3.2272-3.6774$

H $-1.7933-3.1272-6.5985$

H $-2.9515-0.9237-4.1137$

H $-3.8859-1.8723-6.0796$

H $-3.7839-3.4001 \quad-5.1746$

H $-0.2443-1.8020 \quad-7.5042$

H $-0.25390 .4345 \quad-8.5708$

H $-0.5870 \quad 1.1149 \quad-6.9529$

H $6.5872-2.6655-3.8495$

H $-6.1923 \quad 6.1941 \quad-2.9354$

H $-3.4894 \quad 2.6043 \quad-4.0730$

H $8.6811 \quad 3.2280 \quad-2.2865$

H $5.8961 \quad 1.5031 \quad 3.7813$

H $-0.5285-3.1891-1.5288$

H $-0.0357-0.8309-0.4756$

H $-1.4087-1.5492-0.2635$

$\begin{array}{lllll}\mathrm{H} & 0.3223 & 12.5355 & -0.2397\end{array}$

H $-0.2796 \quad 8.4984 \quad 8.0112$

H $\quad 0.9080 \quad-5.8969 \quad-4.2400$

$\begin{array}{llll}\text { C } & 0.2160 & 0.1315 & 2.4422\end{array}$

$\begin{array}{llll}\text { O } & 0.0072 & -0.9942 & 2.0514\end{array}$

$\begin{array}{llll}\mathrm{O} & 0.0445 & 0.9279 & 3.3308\end{array}$

H $2.3122 \quad-2.4750 \quad 9.0759$

H $1.7095 \quad-0.3307 \quad 9.1936$

H $1.0194 \quad 0.4455 \quad-7.3205$

H $-8.4996-0.5802 \quad-5.5912$

H $2.2352 \quad 7.1650 \quad-3.4551$

$\begin{array}{llll}\text { C } & -4.4790 & -5.1451 & -0.6993\end{array}$

H $-9.5192-4.5224-2.2092$

H $-10.5505 \quad-3.1452 \quad-2.0101$

H -7.8144 - $-1.0316-1.4715$

H $-9.3964-1.4244-0.8360$

H $-6.5718-2.7611-1.7872$

H $-7.7423 \quad-5.0577 \quad-3.2381$

H $-7.2243 \quad-5.2449-1.5534$

H $-5.5167-6.0198 \quad-3.1743$

H $-5.5091 \quad-4.4521 \quad-3.9709$

H $-4.4161 \quad-6.2353-0.8049$

H $-5.3776-4.9186 \quad-0.1211$

H $-3.5247-4.6487 \quad-2.5693$

H $-4.5369-3.3863-1.9380$

H $-3.3695-2.4806-0.2346$

H $-3.5086-1.5511 \quad 0.9941$

H -12.3493 $-3.2359 \quad 3.2893$

H -12.0694 -1.7544 2.3505

H $-12.4573 \quad-3.2607 \quad 1.5201$

H $-10.2451 \quad-4.2462 \quad 2.3401$

H $-9.8917-2.7420 \quad 3.1523$

H $-12.5540-1.9630-1.6831$

$\mathrm{H}-11.5510 \quad-2.2227 \quad-0.5157$

H $-2.4051-4.7218 \quad-0.2916$

\section{Int2}

$\begin{array}{llll}\mathrm{N} & -3.3107 & -4.6164 & 0.7034\end{array}$

C $-4.4431-4.7566-1.4706$

C $-5.5565-5.3663-2.3306$

$\begin{array}{llll}\text { C } & -6.9879 & -5.0258 & -1.9053\end{array}$

N $-7.2353-3.5863-1.9593$

C $-8.4770-3.0494 \quad-1.8431$
N $-9.5581-3.8174 \quad-1.9785$

$\mathrm{N}-8.6320 \quad-1.7207 \quad-1.7110$

C - $12.0165 \quad-2.4270 \quad 2.3719$

C - $10.5236-2.7699 \quad 2.3936$

C $-9.7300-2.4421 \quad 1.1206$

$\begin{array}{lllll}\text { O } & -8.6283 & -2.9860 & 0.9407\end{array}$

O $-10.2697 \quad-1.5813 \quad 0.3041$

$\begin{array}{llll}\mathrm{O}-12.0084 & -2.7934 & -1.2391\end{array}$

$\begin{array}{llll}\text { O } & -3.3268 & -1.5930 & 0.0558\end{array}$

$\begin{array}{llll}\text { C } & 6.3856 & -8.6058 & 0.1107\end{array}$

$\begin{array}{llll}\text { C } & 5.2789 & -7.8872 & 0.8665\end{array}$

$\begin{array}{llll}\text { O } & 4.1580 & -8.3637 & 1.0324\end{array}$

N $5.6153 \quad-6.6710 \quad 1.3906$

C $4.7124 \quad-5.9966 \quad 2.3090$

$\begin{array}{llll}\text { C } & 3.6707 & -5.1206 & 1.5997\end{array}$

$\begin{array}{llll}\text { O } & 3.9164 & -4.5105 & 0.5561\end{array}$

C $5.6748 \quad-5.1007 \quad 3.1222$

$\begin{array}{llll}\text { C } & 6.7542 & -4.7290 & 2.0942\end{array}$

$\begin{array}{llll}\text { C } & 6.9194 & -6.0053 & 1.2579\end{array}$

N $2.5076 \quad-4.9573 \quad 2.2760$

$\begin{array}{llll}\text { C } & 1.4730 & -4.0678 & 1.8033\end{array}$

$\begin{array}{llll}\text { C } & 0.1518 & -4.4098 & 2.4787\end{array}$

$\begin{array}{lllll}\text { O } & 0.0860 & -5.2118 & 3.4032\end{array}$

N $-0.9257 \quad-3.7327 \quad 1.9696$

C $-2.1647 \quad-3.6990 \quad 2.7232$

C $-3.4230-4.1768 \quad 1.9896$

$\begin{array}{llll}\text { O } & -4.5101 & -4.0786 & 2.5627\end{array}$

$\begin{array}{llll}\text { C } & -2.4533 & -2.2955 & 3.3515\end{array}$

$\begin{array}{llll}\text { O } & -3.5239 & -1.5733 & 2.7292\end{array}$

$\begin{array}{llll}\text { C } & 8.1182 & -5.7758 & -5.5855\end{array}$

$\begin{array}{llll}\text { C } & 8.1654 & -5.9881 & -4.0664\end{array}$

C $7.4300-4.9552-3.2231$

$\begin{array}{llll}\text { O } & 7.4377 & -3.7377 & -3.7601\end{array}$

O $\quad 6.9151 \quad-5.2188-2.1460$

C $0.1826-5.8366-2.9281$

$\begin{array}{llll}\text { C } & -0.1706 & -5.1389 & -4.2528\end{array}$

$\begin{array}{lllll}\text { O } & 0.2377 & -5.5313 & -5.3407\end{array}$

C $0.4493-4.8912-1.7479$

C $1.5772-3.9102-2.0497$

O $-0.7792 \quad-4.2068$ - 1.4069

$\mathrm{N}-1.0245-4.0652-4.1488$

$\begin{array}{llll}\text { C } & -1.6599 & -3.5017 & -5.3231\end{array}$

C $-1.0900-2.1293-5.7503$

O $-1.4412 \quad-1.0952 \quad-5.1504$

C $-3.1674-3.3281-5.0661$

$\begin{array}{llll}\text { O } & -3.4121 & -2.4598 & -3.9604\end{array}$

N $-0.2911-2.0969-6.8326$

C $0.2146-0.8520-7.4285$

$\begin{array}{llll}\text { C } & -7.9538 & -0.6487 & -5.0923\end{array}$

C $-6.5202-0.9307-4.6502$

$\begin{array}{llll}\mathrm{N} & -5.9766 & 0.1819 & -3.8638\end{array}$

$\begin{array}{llll}\mathrm{C} & -5.4229 & 0.0882 & -2.6542\end{array}$

N $-5.1215-1.1225-2.1326$

N $-5.2267 \quad 1.1992-1.9252$

C $-5.0044 \quad 5.5101-3.9536$

C $-4.6182 \quad 4.0883-4.3430$

O

C $-4.7289 \quad 5.7023-2.4436$

$\begin{array}{llll}C & -3.2487 & 5.8514 & -2.0979\end{array}$

$\mathrm{N}-2.6418 \quad 4.8164 \quad-1.5099$ 
$\begin{array}{llll}\text { O } & -2.6714 & 6.9330 & -2.3503\end{array}$

$\begin{array}{llll}\mathrm{N} & -3.4405 & 3.9347 & -4.9830\end{array}$

$\begin{array}{llll}\mathrm{C} & -2.6916 & 2.6729 & -5.0033\end{array}$

C $-1.3848 \quad 2.8403-4.1923$

$\begin{array}{lllll}\mathrm{C} & -0.8935 & 1.5850 & -3.4734\end{array}$

C $-1.8558 \quad 1.1050-2.4065$

$\begin{array}{llll}\mathrm{N} & -1.9618 & -0.2320 & -2.2719\end{array}$

$\begin{array}{lllll}\text { O } & -2.5534 & 1.8803 & -1.7226\end{array}$

$\begin{array}{lllll}\text { O } & -0.4639 & -1.8419 & -0.0936\end{array}$

$\begin{array}{llll}\text { C } & -1.1057 & 8.9247 & 5.9952\end{array}$

$\begin{array}{llll}\mathrm{C} & -0.4652 & 7.5383 & 5.8562\end{array}$

$\begin{array}{llll}\mathrm{N} & -1.1312 & 6.6773 & 4.8726\end{array}$

C $-0.6800 \quad 6.3346 \quad 3.6502$

$\begin{array}{llll}\mathrm{N} & 0.3468 & 6.9538 & 3.0746\end{array}$

$\begin{array}{llll}\mathrm{N} & -1.3031 & 5.3556 & 2.9786\end{array}$

C $1.1007 \quad 11.6382-2.5560$

$\begin{array}{llll}\text { C } & 1.4641 & 10.2191 & -2.1296\end{array}$

$\begin{array}{lllll}\mathrm{N} & 0.3308 & 9.5756 & -1.4627\end{array}$

C $\quad 0.4213 \quad 8.4421 \quad-0.7409$

$\begin{array}{llll}\mathrm{N} & -0.6969 & 7.8168 & -0.3458\end{array}$

$\begin{array}{llll}N & 1.6079 & 8.0036 & -0.3146\end{array}$

C $2.0104 \quad 5.8125 \quad-3.7303$

C $2.6583 \quad 4.4490 \quad-3.9275$

$\begin{array}{lllll}\text { O } & 2.1222 & 3.3989 & -3.5533\end{array}$

C $0.5072 \quad 5.7506 \quad-3.5122$

$\begin{array}{lllll}\text { O } & 0.0319 & 7.0690 & -3.2785\end{array}$

N $3.8671 \quad 4.5111 \quad-4.5368$

$\begin{array}{llll}\text { C } & 4.8544 & 3.4502 & -4.4670\end{array}$

$\begin{array}{llll}\text { C } & 6.0157 & 3.9557 & -3.5978\end{array}$

O $6.2366 \quad 5.1666-3.5246$

$\begin{array}{lllll}\mathrm{N} & 6.7396 & 2.9916 & -2.9954\end{array}$

$\begin{array}{llll}\text { C } & 7.8160 & 3.3084 & -2.0572\end{array}$

$\begin{array}{llll}\text { C } & 7.4987 & 2.8403 & -0.6225\end{array}$

$\begin{array}{llll}\text { C } & 7.6032 & 1.3098 & -0.4916\end{array}$

$\begin{array}{llll}\text { C } & 6.1372 & 3.3717 & -0.1526\end{array}$

$\begin{array}{llll}\text { C } & 7.5300 & 0.8008 & 0.9495\end{array}$

C $2.0130 \quad 1.9882 \quad 8.8515$

C $1.8440 \quad 2.5880 \quad 7.4486$

$\begin{array}{llll}\text { C } & 0.3629 & 2.5609 & 7.0292\end{array}$

C $2.7447 \quad 1.8515 \quad 6.4509$

C $0.0755 \quad 3.1735 \quad 5.6544$

C $1.1353-1.6662 \quad 9.4745$

$\begin{array}{llll}\text { C } & 1.0177 & -2.6670 & 8.3097\end{array}$

$\begin{array}{llll}\text { C } & 1.0949 & -2.0471 & 6.9279\end{array}$

C $0.0678-1.2158 \quad 6.4596$

C $2.1711-2.3056 \quad 6.0692$

C $0.1147 \quad-0.6636 \quad 5.1791$

$\begin{array}{llll}\text { C } & 2.2191 & -1.7620 & 4.7828\end{array}$

C $1.1883 \quad-0.9377 \quad 4.3276$

$\begin{array}{llll}\text { C } & -3.6565 & -0.4079 & 10.4654\end{array}$

$\begin{array}{llll}\text { C } & -3.8285 & -0.3505 & 8.9444\end{array}$

$\begin{array}{llll}\text { C } & -2.9483 & 0.6904 & 8.2292\end{array}$

$\begin{array}{llll}\text { C } & -3.1289 & 0.5922 & 6.7091\end{array}$

$\begin{array}{llll}\text { C } & -3.2252 & 2.1204 & 8.7084\end{array}$

C $\quad 0.4889 \quad 2.3236 \quad 0.6790$

$\begin{array}{llll}\text { C } & 0.7648 & 4.8209 & 0.4344\end{array}$

$\begin{array}{lllll}\text { O } & 0.6849 & 0.2864 & -0.6422\end{array}$

$\begin{array}{llll}\text { O } & -0.4775 & 5.1219 & 0.2953\end{array}$

O $\quad 1.5730 \quad 5.6032 \quad 1.0276$

C $7.0920-0.3453-4.1347$ $\begin{array}{llll}\text { C } & 5.0842 & -1.6649 & 1.9417\end{array}$

C $3.0928-1.3565-0.1659$

$\begin{array}{llll}\text { C } & 5.0843 & -2.0099 & -1.5670\end{array}$

C 4.1018 - $1.0913-1.2619$

$\begin{array}{llll}\text { C } & 4.0456 & 0.0632 & -2.1049\end{array}$

N $3.0142 \quad 0.9158 \quad-2.0604$

$\begin{array}{llll}\mathrm{N} & 5.0493 & 0.3120 & -2.9904\end{array}$

C $5.9792 \quad-0.6362-3.1642$

N $6.0163 \quad-1.8127 \quad-2.5307$

$\begin{array}{llll}\text { S } & 2.9210 & 1.7338 & 2.4230\end{array}$

$\begin{array}{llll}\text { C } & 2.2673 & 0.6734 & 1.1240\end{array}$

$\begin{array}{llll}\text { N } & 3.1306 & -0.4457 & 0.9851\end{array}$

C $4.1584-0.4936 \quad 1.9353$

$\begin{array}{llll}\text { C } & 4.1972 & 0.5455 & 2.7955\end{array}$

C $\quad 5.0959 \quad 0.7798 \quad 3.9645$

$\begin{array}{llll}\text { C } & 1.1436 & 0.9869 & 0.4247\end{array}$

$\begin{array}{llll}\text { C } & 1.2424 & 3.4783 & -0.0479\end{array}$

H $2.9636 \quad 1.6845 \quad-2.7198$

H $2.1393 \quad 0.6619 \quad-1.5725$

$\begin{array}{llll}\mathrm{H} & -0.5446 & 2.2872 & 0.3228\end{array}$

$\begin{array}{llll}\mathrm{H} & 0.4655 & 2.5454 & 1.7561\end{array}$

H $7.9467 \quad 0.0877 \quad-3.6013$

H $6.7677 \quad 0.3696 \quad-4.8926$

H $7.4361-1.2655-4.6101$

H $4.5330-2.6055 \quad 1.9788$

H $5.7533 \quad-1.6222 \quad 2.8008$

H $5.6972 \quad-1.6926 \quad 1.0370$

H $4.5944 \quad 0.5653 \quad 4.9149$

H $1.0774 \quad 3.3883-1.1271$

H 5.1359 -2.9626 -1.0428

H $2.0861-1.2805-0.5670$

H $\begin{array}{llll}5.9886 & 0.1525 & 3.9052\end{array}$

$\begin{array}{llll}\text { H } & 2.3138 & 3.4022 & 0.1414\end{array}$

H $3.2470 \quad-2.3773 \quad 0.1863$

H $-0.6886 \quad-0.0127 \quad 4.8474$

H $1.2295 \quad-0.5033 \quad 3.3340$

H $-0.7787-1.0037 \quad 7.1055$

H $2.9772 \quad-2.9501 \quad 6.4102$

H $3.0648 \quad-1.9690 \quad 4.1353$

H $\quad 0.0645 \quad-3.2030 \quad 8.4022$

H $\quad 1.8031 \quad-3.4243 \quad 8.4052$

H $0.3492 \quad-0.9059 \quad 9.4235$

$\begin{array}{llll}\mathrm{H} & -3.6057 & -1.3389 & 8.5217\end{array}$

H $-4.8809 \quad-0.1478 \quad 8.7010$

$\begin{array}{llll}\mathrm{H} & -4.2761 & 2.3874 & 8.5413\end{array}$

H $-3.0142 \quad 2.2439 \quad 9.7739$

H $-2.6050 \quad 2.8404 \quad 8.1634$

H $-1.8984 \quad 0.4583 \quad 8.4638$

$\begin{array}{llll}\mathrm{H} & -4.1574 & 0.8427 & 6.4232\end{array}$

H $-2.4574 \quad 1.2803 \quad 6.1844$

H $-2.9242 \quad-0.4192 \quad 6.3431$

H $-1.7787 \quad 4.62913 .4908$

H $-1.0725 \quad 5.1960 \quad 1.9850$

H $\quad 0.7111 \quad 7.8069 \quad 3.4648$

H $\quad 0.8376 \quad 6.4949 \quad 2.2724$

H $-2.0148 \quad 6.2721 \quad 5.1451$

H $\quad 0.5893 \quad 7.6122 \quad 5.5778$

H $-0.4877 \quad 7.0116 \quad 6.8151$

H $-1.0676 \quad 9.4766 \quad 5.0514$

H $-2.1532 \quad 8.8429 \quad 6.2991$
H $\quad-0.5889 \quad 6.8613 \quad 0.0358$

$\mathrm{H}-1.5080 \quad 7.8842-0.9568$

H $-0.5773 \quad 9.7577 \quad-1.8673$

H $2.4538 \quad 8.4360 \quad-0.6433$

H $\quad 1.6731 \quad 7.0994 \quad 0.1821$

H $1.7811 \quad 9.6139 \quad-2.9881$

H $2.2968 \quad 10.2733-1.4211$

H $1.9557 \quad 12.1089 \quad-3.0466$

H $\quad 0.8169 \quad 12.2449 \quad-1.6922$

H $7.2431 \quad-8.7988 \quad 0.7638$

H $7.7167 \quad-6.6431 \quad 1.6623$

H $7.1402 \quad-5.7852 \quad 0.2103$

H $7.6916-4.4115 \quad 2.5575$

H $6.3985 \quad-3.9245 \quad 1.4499$

H $\quad 6.1045 \quad-5.6935 \quad 3.9362$

H $5.1730 \quad-4.2359 \quad 3.5634$

H $4.1986-6.7396 \quad 2.9253$

H $2.2506 \quad-5.5516 \quad 3.0550$

H $1.3786-4.1392 \quad 0.7191$

H $1.7078 \quad-3.0182 \quad 2.0280$

H $-0.7119-2.9729 \quad 1.3088$

H $-2.0239-4.4146 \quad 3.5400$

H $-1.5580-1.6829 \quad 3.2508$

H $-2.6689-2.4046 \quad 4.4181$

H $-4.3126-2.13512 .8584$

H $-4.7208-1.7874 \quad-2.8022$

H $-4.6338-1.1216-1.2317$

H $-4.3390 \quad 1.2490-1.4162$

H $-5.4292 \quad 2.0663 \quad-2.4208$

H $-6.0219 \quad 1.1220-4.2498$

H $-6.4971-1.8273-4.0294$

H $-5.8649-1.1084-5.5100$

$\begin{array}{llll}\mathrm{H} & -8.0182 & 0.2762 & -5.6748\end{array}$

H $-8.6087-0.5722 \quad-4.2212$

H $-1.2513-0.8118-2.6956$

H $-2.3882-0.5932 \quad-1.4251$

H $-0.6974 \quad 0.7736-4.1772$

H $\quad 0.0501 \quad 1.8075 \quad-2.9631$

H $-0.5809 \quad 3.2007-4.8398$

H $-1.5588 \quad 3.6094 \quad-3.4385$

H $-2.4948 \quad 2.3665-6.0349$

H $-2.9129 \quad 4.7757 \quad-5.1748$

H $-4.4579 \quad 6.2633-4.5288$

H $-5.1828 \quad 4.8770 \quad-1.8886$

$\mathrm{H} \quad-5.2048 \quad 6.6305-2.1171$

H $-1.7060 \quad 4.9064-1.1012$

$\begin{array}{llll}\mathrm{H} & -3.0325 & 3.8807 & -1.4774\end{array}$

H $7.7242 \quad-0.2752 \quad 0.9898$

$\begin{array}{llll}\mathrm{H} & 8.2757 & 1.2969 & 1.5815\end{array}$

H $\quad 6.5431 \quad 0.9726 \quad 1.3865$

H $\quad 6.8015 \quad 0.8388-1.0717$

H $8.5493 \quad 0.9825 \quad-0.9428$

H $5.3186 \quad 2.8913-0.6991$

$\begin{array}{llll}\mathrm{H} & 5.9740 & 3.1750 & 0.9096\end{array}$

H $6.0595 \quad 4.4518 \quad-0.3145$

$\begin{array}{llll}\mathrm{H} & 8.2822 & 3.2785 & 0.0132\end{array}$

H $7.9395 \quad 4.3920-2.0936$

$\begin{array}{llll}\mathrm{H} & 6.3303 & 2.0592 & -2.9914\end{array}$

H $\quad \begin{array}{llll}5.2403 & 3.2269 & -5.4690\end{array}$

H $4.4148 \quad 2.5343 \quad-4.0757$ 
H $\quad \begin{array}{llll}4.3102 & 5.4245 & -4.5712\end{array}$

$\begin{array}{lllll}\mathrm{H} & 2.4713 & 6.2721 & -2.8453\end{array}$

H $\quad 0.0291 \quad 5.3121 \quad-4.3996$

H $0.2902 \quad 5.0856-2.6671$

H $-0.9096 \quad 6.9976-3.0286$

$\begin{array}{llll}\text { H } & 6.7362 & -7.9968 & -0.7297\end{array}$

H $5.9901-9.5513-0.2580$

H $8.6835-6.5603-6.0967$

H 7.0888 -5.8038 -5.9552

H $8.5437 \quad-4.8070 \quad-5.8556$

H $7.7634-6.9650-3.7846$

H $9.2094 \quad-5.9755 \quad-3.7247$

H $\quad 0.5410 \quad 2.5990 \quad 4.8481$

H $-1.0057 \quad 3.1996 \quad 5.4625$

$\begin{array}{llll}\mathrm{H} & 0.4553 & 4.2011 & 5.5917\end{array}$

H $2.6922 \quad 2.2780 \quad 5.4460$

H $3.7895 \quad 1.8948 \quad 6.7754$

H $2.4582 \quad 0.7977 \quad 6.3745$

$\begin{array}{llll}\mathrm{H} & 0.0170 & 1.5212 & 7.0361\end{array}$

H $-0.2257 \quad 3.0873 \quad 7.7923$

H $2.1609 \quad 3.6424 \quad 7.4837$

H $1.38892 .5027 \quad 9.5908$

H $3.0535 \quad 2.0512 \quad 9.1861$

H 1.0418 -2.1811 10.4354

H $-4.2477-1.2222 \quad 10.8946$

H $-2.6083 \quad-0.5792 \quad 10.7371$

H $-3.9772 \quad 0.5199 \quad 10.9482$

H $1.3476-3.3048-2.9316$

H $1.7356-3.2386-1.2092$

H $2.5143-4.4462-2.2182$

$\begin{array}{lllll}\mathrm{H} & 0.7456 & -5.5171 & -0.8950\end{array}$

H $-0.6474-6.5076-2.6751$

H $-1.3306-3.8001-3.2142$

$\begin{array}{llll}\mathrm{H} & -1.5123 & -4.2221 & -6.1333\end{array}$

H $-2.8600-1.6666-4.1393$

H $-3.6569-2.9525 \quad-5.9763$

H $-3.5979-4.3011-4.8176$

$\begin{array}{lllll}\text { H } & 0.0457 & -2.9827 & -7.1869\end{array}$

H $0.3036-0.9856-8.5076$

H $-0.4985-0.0576-7.2089$

$\begin{array}{llll}\text { H } & 6.9317 & -3.0821 & -3.1719\end{array}$

$\begin{array}{llll}\mathrm{H} & -6.0728 & 5.6325 & -4.1417\end{array}$

$\begin{array}{llll}\mathrm{H} & -3.3466 & 1.9251 & -4.5543\end{array}$

H $\quad 8.7484 \quad 2.8463 \quad-2.4038$

H $5.4344 \quad 1.8220 \quad 3.9998$

H $-0.5613-3.3393-0.9762$

H $\quad 0.0728 \quad-0.9315-0.3021$

H -1.3846 $-1.5512 \quad 0.0050$

H $\quad 0.2697 \quad 11.6360 \quad-3.2692$

$\begin{array}{llll}\mathrm{H} & -0.5761 & 9.5024 & 6.7574\end{array}$

$\begin{array}{llll}\text { H } & 1.0628 & -6.4528 & -3.1178\end{array}$

H $2.1013 \quad-1.1546 \quad 9.4547$

H $1.7272 \quad 0.9338 \quad 8.8464$

$\begin{array}{llll}\text { H } & 1.1894 & -0.5772 & -7.0136\end{array}$

$\begin{array}{llll}\mathrm{H} & -8.3115 & -1.4674 & -5.7228\end{array}$

H $2.2363 \quad 6.4680 \quad-4.5778$

$\begin{array}{llll}\text { C } & -4.4787 & -5.1766 & 0.0007\end{array}$

H $-9.4768-4.7981-1.7629$

H $-10.4977-3.3993-1.8227$

H $-7.7551 \quad-1.2450-1.5347$
H $-9.3639-1.5135-0.9237$

H $-6.5168-3.0034-1.5511$

H $-7.6749-5.5140 \quad-2.6029$

$\begin{array}{llll}\mathrm{H} & -7.2019 & -5.4084 & -0.8980\end{array}$

H $-5.4625-6.4582-2.3232$

H $-5.4176 \quad-5.0445-3.3688$

$\begin{array}{llll}\mathrm{H} & -4.4790 & -6.2702 & 0.0894\end{array}$

H $-5.3609-4.8012 \quad 0.5232$

H $-3.4733-5.0291-1.9015$

H $-4.4781 \quad-3.6632 \quad-1.5271$

$\begin{array}{llll}\mathrm{H} & -3.2940 & -2.5663 & 0.0091\end{array}$

H $-3.4320-1.4197 \quad 1.0307$

H -12.4914 $-2.6972 \quad 3.3201$

H -12.1645 $-1.3575 \quad 2.2012$

H $-12.5321 \quad-2.9615 \quad 1.5678$

$\mathrm{H}-10.3618 \quad-3.8326 \quad 2.6021$

H $-10.0259-2.2326 \quad 3.2123$

H $-12.4882-2.1639-1.7907$

H $-11.5461-2.2439-0.5486$

H $-2.3783 \quad-4.7823 \quad 0.3260$

\section{Int3}

C $7.8274 \quad-7.4513 \quad 1.3254$

C 6.5008 -6.9366 1.8552

$\begin{array}{llll}\text { O } & 5.4791 & -7.6182 & 1.8664\end{array}$

N $6.5083 \quad-5.6675 \quad 2.3679$

C $5.3528 \quad-5.1961 \quad 3.1181$

C $4.3152-4.5199 \quad 2.2202$

$\begin{array}{lllll}\text { O } & 4.6367 & -3.7223 & 1.3293\end{array}$

C $5.9771-4.1610 \quad 4.0789$

C $7.1300 \quad-3.5630 \quad 3.2572$

C $7.6566-4.7512 \quad 2.4335$

$\begin{array}{llll}\text { N } & 3.0238 & -4.7445 & 2.5252\end{array}$

C $1.9732-4.0600 \quad 1.8166$

C $0.6306-4.6718 \quad 2.1896$

$\begin{array}{llll}\mathrm{O} & 0.5298 & -5.5273 & 3.0603\end{array}$

N $-0.4103-4.1662 \quad 1.4730$

C $-1.7815 \quad-4.4929 \quad 1.8091$

C $-2.5916 \quad-4.9633 \quad 0.6046$

$\begin{array}{llll}\text { O } & -3.8379 & -4.9016 & 0.6562\end{array}$

C $-2.5355-3.3248 \quad 2.4854$

$\begin{array}{llll}\text { O } & -3.6614 & -3.7625 & 3.2067\end{array}$

N $-1.9275 \quad-5.4200 \quad-0.4668$

C $-2.6174-5.9097-1.6534$

$\begin{array}{llll}\text { C } & -2.3111 & -5.0608 & -2.8895\end{array}$

$\begin{array}{llll}C & -3.0708 & -5.5131 & -4.1437\end{array}$

C $-4.5914 \quad-5.6331 \quad-3.9899$

N $-5.1841-4.3866-3.5164$

$\begin{array}{llll}\mathrm{C} & -6.5374 & -4.2749 & -3.2926\end{array}$

$\mathrm{N}-7.4089-5.1229-3.8240$

N $-6.9665-3.2361 \quad-2.5783$

C $10.8855-4.0487-3.1882$

$\begin{array}{llll}\text { C } & 9.9624 & -4.0779 & -1.9714\end{array}$

C $9.1481-2.8066-1.8202$

O $9.1758-1.8936-2.6296$

O $8.4193-2.7855-0.7005$

$\begin{array}{llll}\text { C } & 2.6364 & -5.3973 & -3.2412\end{array}$

C $2.6266-4.6655-4.5839$

$\begin{array}{llll}\text { O } & 3.5675 & -4.6987 & -5.3694\end{array}$ $\begin{array}{llll}\text { C } & 2.2094 & -4.5337 & -2.0425\end{array}$

C $3.0047-3.2347-1.9391$

$\begin{array}{lllll}\text { O } & 0.7968 & -4.2844 & -2.1491\end{array}$

N $1.4673 \quad-3.9966-4.8841$

C $1.3742 \quad-3.2423-6.1039$

C $2.1137-1.8846-6.0392$

$\begin{array}{llll}\text { O } & 2.2283 & -1.2300 & -4.9959\end{array}$

C $-0.1096-3.0710-6.5018$

O $-0.9693-2.7120 \quad-5.4307$

N $2.5782 \quad-1.4323 \quad-7.2282$

C $3.2650-0.1535-7.4042$

C $-6.0329-1.7364-7.4034$

$\begin{array}{llll}\text { C } & -4.8906 & -1.9292 & -6.3962\end{array}$

$\begin{array}{llll}\mathrm{N} & -4.5615 & -0.6732 & -5.7077\end{array}$

C $-3.9184-0.5893-4.5337$

$\begin{array}{llll}\mathrm{N} & -3.8442 & 0.5985 & -3.8972\end{array}$

$\begin{array}{llll}\mathrm{N} & -3.3937 & -1.6624 & -3.9481\end{array}$

C $-4.29494 .4700 \quad-5.7109$

C $-3.3642 \quad 3.3288-6.0928$

$\begin{array}{lllll}\text { O } & -3.8039 & 2.1788 & -6.2529\end{array}$

C $-4.4909 \quad 4.4570-4.1775$

C $-3.2540 \quad 4.9236-3.4063$

$\mathrm{N}-2.8250 \quad 4.1265 \quad-2.4122$

$\begin{array}{lllll}\mathrm{O} & -2.7276 & 6.0161 & -3.6796\end{array}$

$\begin{array}{llll}\mathrm{N} & -2.0462 & 3.6188 & -6.1363\end{array}$

$\begin{array}{llll}\text { C } & -0.9995 & 2.5907 & -6.1283\end{array}$

$\begin{array}{llll}C & 0.0474 & 2.9015 & -5.0444\end{array}$

C $\quad 0.7182 \quad 1.6636-4.4482$

$\begin{array}{llll}\text { C } & -0.2748 & 0.8437 & -3.6349\end{array}$

$\begin{array}{llll}\mathrm{N} & -0.0432 & -0.4921 & -3.5685\end{array}$

$\begin{array}{lllll}\mathrm{O} & -1.2529 & 1.3756 & -3.0954\end{array}$

C $-11.6349-6.1366-0.7948$

C $-11.4108-4.6756-1.1941$

C $-10.0803-4.4380-1.9160$

$\begin{array}{lllll}\text { O } & -9.7385 & -5.3141 & -2.7728\end{array}$

$\begin{array}{llll}\text { O } & -9.4359 & -3.3949 & -1.6200\end{array}$

$\begin{array}{llll}\text { C } & -3.8781 & 8.6665 & 5.1471\end{array}$

$\begin{array}{llll}\mathrm{C} & -3.6470 & 7.1491 & 5.1444\end{array}$

$\begin{array}{llll}\mathrm{N} & -3.9011 & 6.4961 & 3.8532\end{array}$

$\begin{array}{llll}\text { C } & -3.0018 & 6.3391 & 2.8619\end{array}$

N $-1.8145 \quad 6.9420 \quad 2.9111$

$\begin{array}{llll}\mathrm{N} & -3.3138 & 5.5842 & 1.8010\end{array}$

$\begin{array}{llll}\text { C } & -0.1453 & 12.0258 & -2.5717\end{array}$

C $0.1174 \quad 10.8531 \quad-1.6381$

$\begin{array}{llll}\mathrm{N} & -0.7166 & 9.6964 & -1.9817\end{array}$

$\begin{array}{llll}\text { C } & -0.7744 & 8.5825 & -1.2227\end{array}$

$\begin{array}{lllll}\mathrm{N} & 0.0056 & 8.4901 & -0.1408\end{array}$

$\mathrm{N}-1.6131 \quad 7.5890 \quad-1.5159$

$\begin{array}{llll}\text { C } & 2.3665 & 6.3545 & -3.1826\end{array}$

C $3.2653 \quad 5.1336-3.0296$

$\begin{array}{lllll}\text { O } & 2.8165 & 4.0099 & -2.7733\end{array}$

$\begin{array}{llll}\text { C } & 0.8993 & 6.0307 & -3.4378\end{array}$

N $4.5836 \quad 5.4014 \quad-3.1977$

$\begin{array}{llll}\text { C } & 5.6417 & 4.5099 & -2.7602\end{array}$

C $6.2683 \quad 5.0749-1.4756$

$\begin{array}{lllll}\text { O } & 6.2574 & 6.2917 & -1.2806\end{array}$

N $6.8364 \quad 4.1527 \quad-0.6732$

$\begin{array}{llll}\text { C } & 7.5003 & 4.5079 & 0.5778\end{array}$

$\begin{array}{llll}\text { C } & 6.8118 & 3.9267 & 1.8291\end{array}$

C $7.0572 \quad 2.4138 \quad 1.9831$ 
$\begin{array}{llll}\text { C } & 5.3177 & 4.2782 & 1.8648\end{array}$

$\begin{array}{llll}\text { C } & 6.6771 & 1.8769 & 3.3648\end{array}$

$\begin{array}{llll}\text { C } & -1.0989 & 1.7814 & 8.9748\end{array}$

$\begin{array}{llll}\text { C } & -0.7340 & 2.1511 & 7.5255\end{array}$

C $-1.8542 \quad 2.9883 \quad 6.8791$

$\begin{array}{llll}C & -0.4103 & 0.9030 & 6.6946\end{array}$

$\begin{array}{llll}C & -1.5125 & 3.5538 & 5.4951\end{array}$

C $-1.7183-1.9695 \quad 8.9362$

C $-1.2546-3.1373 \quad 8.0546$

$\begin{array}{llll}\text { C } & -0.2199 & -2.7278 & 7.0293\end{array}$

$\begin{array}{llll}\text { C } & -0.5290 & -2.7175 & 5.6655\end{array}$

$\begin{array}{llll}\text { C } & 1.0672 & -2.3317 & 7.4214\end{array}$

$\begin{array}{llll}\text { C } & 0.4213 & -2.3297 & 4.7202\end{array}$

$\begin{array}{llll}\text { C } & 2.0139 & -1.9289 & 6.4797\end{array}$

C $1.6935-1.9254 \quad 5.1202$

$\begin{array}{llll}C & -6.9156 & -2.3870 & 8.2476\end{array}$

$\begin{array}{llll}\text { C } & -5.7769 & -2.3150 & 7.2200\end{array}$

$\begin{array}{llll}C & -5.4772 & -0.9044 & 6.6759\end{array}$

$\begin{array}{llll}\text { C } & -4.2024 & -0.9057 & 5.8201\end{array}$

$\begin{array}{llll}\text { C } & -6.6461 & -0.3199 & 5.8710\end{array}$

C $-1.0691 \quad-1.6143-0.6681$

$\begin{array}{llll}\text { C } & -6.6230 & 1.6552 & 2.7220\end{array}$

$\begin{array}{llll}\text { O } & -7.0894 & 0.5489 & 2.1298\end{array}$

$\begin{array}{llll}\text { C } & -5.1332 & 1.8118 & 2.5576\end{array}$

$\begin{array}{llll}\text { O } & -7.3073 & 2.4496 & 3.3325\end{array}$

$\begin{array}{llll}\text { C } & -4.5161 & 2.8159 & 3.2076\end{array}$

$\begin{array}{llll}\text { O } & -4.5880 & 0.8714 & 1.7644\end{array}$

C $5.5241-1.0355-0.0960$

$\begin{array}{llll}\text { C } & 4.4178 & -0.2047 & -0.1005\end{array}$

$\begin{array}{llll}\text { C } & 4.5760 & 1.0258 & -0.7976\end{array}$

$\begin{array}{llll}\mathrm{N} & 3.5447 & 1.8695 & -0.9963\end{array}$

$\begin{array}{llll}\mathrm{N} & 5.7921 & 1.3920 & -1.2712\end{array}$

C $6.81110 .5316-1.1558$

$\begin{array}{llll}\mathrm{N} & 6.7180 & -0.6920 & -0.6207\end{array}$

C $-1.4796-0.6196 \quad 0.3803$

$\begin{array}{lllll}\text { O } & 0.0578 & -2.1874 & -0.5330\end{array}$

$\begin{array}{llll}\mathrm{S} & 1.7194 & 2.0194 & 3.2825\end{array}$

C $\quad 1.7491 \quad 1.1665 \quad 1.7197$

C $8.1595 \quad 0.9718 \quad-1.6399$

$\begin{array}{llll}\text { C } & -0.7101 & -0.5282 & 1.5034\end{array}$

$\begin{array}{llll}\text { O } & -1.8807 & -1.8752 & -1.6145\end{array}$

$\begin{array}{llll}C & -0.0288 & 2.7077 & 0.8631\end{array}$

$\begin{array}{llll}\text { C } & -1.2157 & -0.0313 & 2.7685\end{array}$

$\begin{array}{llll}\mathrm{N} & 2.7561 & 0.2052 & 1.7436\end{array}$

$\begin{array}{lllll}\mathrm{O} & 0.1787 & 6.1310 & 1.2078\end{array}$

$\begin{array}{lllll}\text { O } & -3.2551 & 0.5642 & -0.8231\end{array}$

$\begin{array}{llll}\text { C } & 3.4448 & 0.1214 & 2.9731\end{array}$

$\begin{array}{llll}C & 4.5537 & -0.8650 & 3.1387\end{array}$

$\begin{array}{llll}\text { C } & -2.4103 & 0.5685 & 2.8374\end{array}$

$\begin{array}{llll}\text { O } & -1.3168 & 5.1864 & -0.1622\end{array}$

$\begin{array}{llll}\text { C } & 3.0163 & 0.9880 & 3.9132\end{array}$

$\begin{array}{llll}\text { C } & 3.4180 & 1.1417 & 5.3437\end{array}$

$\begin{array}{llll}\text { C } & -3.1567 & 0.8787 & 1.5734\end{array}$

$\begin{array}{lllll}\mathrm{O} & 0.8970 & 0.9820 & -0.5267\end{array}$

$\begin{array}{llll}\text { C } & -2.9022 & -0.0926 & 0.4038\end{array}$

$\begin{array}{llll}\text { C } & 0.8368 & 1.4915 & 0.7567\end{array}$

$\begin{array}{llll}\text { C } & 3.1327 & -0.6252 & 0.5777\end{array}$

$\begin{array}{llll}\text { C } & 0.6876 & 3.9647 & 0.3212\end{array}$

$\begin{array}{llll}\text { C } & -0.1951 & 5.1775 & 0.4562\end{array}$

H $8.2443 \quad-6.7833 \quad 0.5651$
H $6.3616 \quad-4.6900 \quad 4.9566$

H $\quad 5.2540 \quad-3.4192 \quad 4.4254$

H $7.9093 \quad-3.1194 \quad 3.8816$

H $6.7506 \quad-2.7946 \quad 2.5820$

H $\quad 8.5052 \quad-5.2370 \quad 2.9312$

H $7.9746-4.4351 \quad 1.4354$

H $4.8932-6.0402 \quad 3.6375$

H $\quad \begin{array}{llll}1.9531 & -2.9928 & 2.0750\end{array}$

H $2.1352-4.1125 \quad 0.7372$

H $-1.8667-2.8317 \quad 3.1890$

H $-2.8049-2.5816 \quad 1.7238$

$\begin{array}{llll}\mathrm{H} & -4.2114 & -4.2315 & 2.5549\end{array}$

H $-1.7387 \quad-5.3146 \quad 2.5320$

H $-2.5232-4.0177-2.6471$

H $-1.2371 \quad-5.0919-3.0900$

H $-2.7015 \quad-6.4949-4.4636$

H $-2.8345-4.8208-4.9614$

H $-4.8375-6.4707 \quad-3.3199$

H $-5.0150 \quad-5.8767 \quad-4.9712$

H $-4.6582-3.8825 \quad-2.8011$

H $-8.4408 \quad-5.1564 \quad-3.4181$

H $-7.0483-5.9443-4.2821$

H $-6.2635-2.7531-2.0293$

H $-7.9782 \quad-3.2473 \quad-2.2418$

H $-3.6803-5.9003-1.4096$

H $10.3108-3.9013-4.1054$

H $11.4449-4.9847 \quad-3.2730$

H $10.5233-4.2271-1.0409$

H $9.2547-4.9151-2.0255$

H $2.3941-5.1336-1.1387$

H $2.6376 \quad-2.6403 \quad-1.1019$

H $4.0624-3.4473-1.7629$

H $2.9091-2.6371-2.8500$

H $\quad 0.5525 \quad-3.4833-1.6258$

H $1.9689-6.2645 \quad-3.3046$

H $-0.4734-4.0399-6.8525$

H $-0.1798-2.3569-7.3351$

H $-0.7302-1.8355-5.0783$

H $1.8595 \quad-3.8291 \quad-6.8925$

H $3.4308 \quad 0.2729-6.4150$

H $2.6623 \quad 0.5363 \quad-8.0042$

H $-6.9438-1.4064-6.8978$

H $-5.7668-1.0009-8.1696$

$\begin{array}{llll}\mathrm{H} & -5.1800 & -2.6587 & -5.6327\end{array}$

H $-3.9975-2.3155-6.9068$

H $-4.7157 \quad 0.2055-6.1897$

H $-3.0488 \quad 0.7141 \quad-3.2621$

H $-4.0131 \quad 1.3907-4.5097$

H $-2.9069-1.5678 \quad-3.0532$

H $-3.1774-2.4793 \quad-4.4981$

H $-4.8197 \quad 3.4671 \quad-3.8465$

H $-5.2900 \quad 5.1606 \quad-3.9211$

H $-3.1533 \quad 3.1795 \quad-2.2917$

H $-2.0647 \quad 4.4221 \quad-1.7997$

H $-3.8973 \quad 5.4439-6.0083$

H $\quad 0.8174 \quad 3.5686 \quad-5.4427$

H $-0.4525 \quad 3.4225 \quad-4.2252$

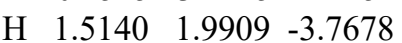

H $\quad 1.1950 \quad 1.0313 \quad-5.2016$

H $-0.6040-1.0358-2.9147$
H $0.8634 \quad-0.8640 \quad-3.8643$

H $-0.5393 \quad 2.5104 \quad-7.1195$

H $-1.7771 \quad 4.5659 \quad-5.9031$

H $-12.6220-6.2788-0.3428$

H -11.5515 $-6.7805-1.6729$

$\mathrm{H}-12.2090-4.3562-1.8780$

H $-11.4503-4.0097-0.3275$

H $-4.9110 \quad 8.9040 \quad 4.8788$

$\mathrm{H} \quad-3.2209 \quad 9.1776 \quad 4.4365$

H $-4.3029 \quad 6.6645 \quad 5.8716$

H $-2.6252 \quad 6.9003 \quad 5.4482$

$\begin{array}{llll}\mathrm{H} & -4.8377 & 6.1681 & 3.6675\end{array}$

H $-1.6119 \quad 7.5988 \quad 3.6456$

H $-1.0538 \quad 6.6610 \quad 2.2606$

H $-4.0230 \quad 4.8668 \quad 1.9015$

$\mathrm{H}-2.6287 \quad 5.4683 \quad 1.0380$

H $-1.1860 \quad 12.3581-2.5068$

H $\quad 0.4934 \quad 12.8674 \quad-2.2961$

H $-0.096111 .1606-0.6055$

H $1.173210 .5598-1.6912$

H $-1.4159 \quad 9.8063 \quad-2.7010$

H $-1.5642 \quad 6.7325-0.9416$

H $-1.9927 \quad 7.4278 \quad-2.4486$

$\begin{array}{llll}\mathrm{H} & 0.0657 & 7.6056 & 0.3988\end{array}$

$\begin{array}{llll}\mathrm{H} & 0.6105 & 9.2514 & 0.1142\end{array}$

$\begin{array}{lllll}\mathrm{H} & 0.4923 & 5.3873 & -2.6556\end{array}$

$\begin{array}{lllll}\mathrm{H} & 0.3046 & 6.9463 & -3.4712\end{array}$

$\begin{array}{lllll}\mathrm{H} & 0.7715 & 5.5148 & -4.3900\end{array}$

H $2.4645 \quad 6.9240 \quad-2.2471$

H $\begin{array}{llll}5.2605 & 3.4981 & -2.6361\end{array}$

H $\quad 6.4240 \quad 4.4694 \quad-3.5271$

H $7.3016 \quad 4.4189 \quad 2.6824$

H $4.8639 \quad 3.9970 \quad 2.8190$

H $5.1638 \quad 5.3523 \quad 1.7194$

$\begin{array}{llll}\mathrm{H} & 4.7701 & 3.7519 & 1.0759\end{array}$

$\begin{array}{llll}\mathrm{H} & 6.4943 & 1.8699 & 1.2157\end{array}$

$\begin{array}{llll}\mathrm{H} & 8.1186 & 2.2048 & 1.7970\end{array}$

$\begin{array}{llll}\mathrm{H} & 5.6035 & 1.9720 & 3.5467\end{array}$

H $6.9386 \quad 0.8191 \quad 3.4631$

H $7.2047 \quad 2.4227 \quad 4.1548$

$\begin{array}{llll}\mathrm{H} & 7.4981 & 5.5990 & 0.6182\end{array}$

$\begin{array}{llll}\mathrm{H} & 0.1694 & 2.7797 & 7.5594\end{array}$

$\begin{array}{lllll}\text { H } & 0.3489 & 0.2811 & 7.1773\end{array}$

$\begin{array}{llll}\mathrm{H} & -1.2997 & 0.2771 & 6.5615\end{array}$

H $-0.0392 \quad 1.1590 \quad 5.6968$

H $-2.1059 \quad 3.8187 \quad 7.5520$

H $-2.7596 \quad 2.3691 \quad 6.8068$

H $-2.3226 \quad 4.1932 \quad 5.1236$

H $-0.5978 \quad 4.1574 \quad 5.5273$

H $-1.3493 \quad 2.7623 \quad 4.7570$

H $-1.2615 \quad 2.6761 \quad 9.5849$

H $-0.8416 \quad-3.9238 \quad 8.6979$

H $-2.1153 \quad-3.5778 \quad 7.5403$

H $-1.5189-3.0334 \quad 5.3490$

H $1.3293 \quad-2.3419 \quad 8.4762$

H $\quad 0.1626 \quad-2.3517 \quad 3.6684$

H $3.0054-1.6276 \quad 6.8056$

H $2.4252 \quad-1.6138 \quad 4.3840$

H $-2.1560-1.1643 \quad 8.3401$

H $-4.8597-2.7052 \quad 7.6811$ 
$\begin{array}{llll}\text { H } & -5.9950 & -2.9809 & 6.3740\end{array}$

$\begin{array}{llll}\text { H } & -5.3022 & -0.2487 & 7.5429\end{array}$

H $-4.3151-1.5416 \quad 4.9339$

$\begin{array}{llll}\mathrm{H} & -3.3391 & -1.2740 & 6.3830\end{array}$

H $-3.9655 \quad 0.1117 \quad 5.4832$

$\begin{array}{llll}\mathrm{H} & -7.5703 & -0.2817 & 6.4543\end{array}$

H $-6.4244 \quad 0.7015 \quad 5.5410$

H $-6.8373-0.9246 \quad 4.9759$

H $-7.8774-2.0985 \quad 7.8126$

H $-5.1294 \quad 3.4415 \quad 3.8430$

$\begin{array}{llll}\mathrm{H} & -3.4450 & 2.9512 & 3.2181\end{array}$

H $5.4606 \quad-2.0317 \quad 0.3344$

H $2.5996 \quad 1.5188-0.9011$

$\begin{array}{llll}\text { H } & 0.0290 & 0.5489 & -0.6847\end{array}$

H $\quad 8.0745 \quad 1.8575 \quad-2.2702$

H $8.7952 \quad 1.2153 \quad-0.7800$

$\begin{array}{llll}\mathrm{H} & 8.6370 & 0.1525 & -2.1835\end{array}$

H $\quad 0.2236-1.0787 \quad 1.5159$

H $-0.3380 \quad 2.8639 \quad 1.9031$

$\begin{array}{llll}\mathrm{H} & -0.9360 & 2.5192 & 0.2799\end{array}$

$\begin{array}{llll}\mathrm{H} & -0.6187 & -0.2003 & 3.6579\end{array}$

H $-4.2221 \quad 0.5474-0.8607$

H $4.8272 \quad-0.9460 \quad 4.1904$

H $4.2832-1.8566 \quad 2.7758$

H $5.4435 \quad-0.5575 \quad 2.5838$

H $-2.8388 \quad 0.8567 \quad 3.7883$

H $2.6388 \quad 0.7635 \quad 6.0163$

$\begin{array}{llll}\mathrm{H} & 4.3396 & 0.5950 & 5.5518\end{array}$

H $-2.8742 \quad 1.8826 \quad 1.2211$

$\begin{array}{llll}\mathrm{H} & -3.5648 & -0.9612 & 0.5280\end{array}$

$\begin{array}{llll}\text { H } & 3.2593 & -1.6543 & 0.9140\end{array}$

H $2.2858-0.6114-0.1043$

$\begin{array}{llll}\mathrm{H} & 1.6155 & 4.1330 & 0.8707\end{array}$

$\begin{array}{lllll}\mathrm{H} & 0.9350 & 3.8009 & -0.7341\end{array}$

H - $10.8823 \quad-6.4651 \quad-0.0700$

H $-3.6822 \quad 9.0690 \quad 6.1445$

H -2.01641 .18229 .0066$

$\begin{array}{llll}\mathrm{H} & -0.3037 & 1.1926 & 9.4424\end{array}$

$\begin{array}{llll}\mathrm{H} & -7.0251 & -3.4026 & 8.6396\end{array}$

H $-6.7214-1.7208 \quad 9.0960$

H $-2.4617-2.2977 \quad 9.6693$

H $-0.8746-1.5388 \quad 9.4846$

H $11.5989-3.2241-3.1153$

H $7.6629 \quad-8.4383 \quad 0.8955$

H $\quad 8.5617 \quad-7.5269 \quad 2.1347$

$\begin{array}{llll}\text { H } & -6.2408 & -2.6849 & -7.9037\end{array}$

H $3.6347 \quad 2.6506-1.6405$

H $2.7679 \quad 6.9990 \quad-3.9728$

H $7.8638-1.9371-0.6816$

$\begin{array}{llll}\text { O } & -4.4262 & -2.7552 & -1.0681\end{array}$

H $-3.5093-2.4269-1.1673$

H $-4.3808-3.4819-0.4159$

H $3.5985 \quad 2.1924 \quad 5.5964$

H $\quad 8.5443 \quad 4.1730 \quad 0.5406$

H $\quad 0.0747 \quad 11.7626 \quad-3.6107$

H $-8.0449 \quad 0.5103 \quad 2.3073$

H $2.5644 \quad-2.0717-8.0093$

H $\quad 4.2295 \quad-0.3069 \quad-7.8941$

$\begin{array}{llll}\text { H } & 3.6537 & -5.7629 & -3.0920\end{array}$

H $0.7836 \quad-3.8626-4.1440$
H $2.7255 \quad-5.4488 \quad 3.1908$

$\begin{array}{lllll}\mathrm{H} & -0.2191 & -3.4268 & 0.7886\end{array}$

H $-0.9186 \quad-5.3287-0.4954$

H $\quad 4.8570 \quad 6.3763 \quad-3.1414$

H $6.6068 \quad 3.1776-0.8688$

H $-2.3279-6.9535-1.8263$

H $-5.2516 \quad 4.3059 \quad-6.2085$

H $-1.5115 \quad 1.6476 \quad-5.9263$

\section{TS4}

C $7.7372 \quad-7.5967 \quad 0.9508$

C $6.4326-7.0565 \quad 1.5131$

$\begin{array}{llll}\text { O } & 5.3869 & -7.6998 & 1.5038\end{array}$

$\begin{array}{llll}\mathrm{N} & 6.4857 & -5.8098 & 2.0787\end{array}$

$\begin{array}{llll}\text { C } & 5.3506 & -5.3312 & 2.8572\end{array}$

C $4.3215-4.59351 .9992$

$\begin{array}{llll}\text { O } & 4.6576 & -3.7262 & 1.1789\end{array}$

C $6.0151-4.3456 \quad 3.8426$

$\begin{array}{llll}C & 7.1730 & -3.7514 & 3.0250\end{array}$

C $7.6587-4.9276 \quad 2.1596$

$\begin{array}{llll}\mathrm{N} & 3.0271 & -4.8413 & 2.2611\end{array}$

C $1.9873-4.1189 \quad 1.5708$

C $0.6390 \quad-4.7435 \quad 1.8984$

$\begin{array}{lllll}\text { O } & 0.5381 & -5.6329 & 2.7377\end{array}$

$\begin{array}{llll}\mathrm{N} & -0.3977 & -4.2037 & 1.2068\end{array}$

C $-1.7630 \quad-4.5259 \quad 1.5681$

$\begin{array}{llll}C & -2.6027 & -5.0290 & 0.3979\end{array}$

$\begin{array}{llll}\text { O } & -3.8477 & -5.0439 & 0.5209\end{array}$

C $-2.4856-3.3276 \quad 2.2290$

$\begin{array}{llll}\text { O } & -3.6209 & -3.7268 & 2.9618\end{array}$

$\mathrm{N}-1.9760 \quad-5.4159 \quad-0.7201$

C $-2.6973-5.8143 \quad-1.9281$

C $-2.3825-4.8568-3.0808$

C $-3.1914-5.1128-4.3571$

C $-4.7060 \quad-5.2369-4.1586$

N $-5.2420 \quad-4.0897 \quad-3.4262$

C $-6.6038-3.9411 \quad-3.2164$

N $-7.4992-4.6017-3.9388$

N $-6.9946 \quad-3.0453 \quad-2.3172$

C $10.8203-4.0117-3.4017$

C $9.9547-4.0463-2.1452$

C $9.1430-2.7801-1.9600$

O $9.1410-1.8590 \quad-2.7600$

$\begin{array}{lllll}\text { O } & 8.4323 & -2.7825 & -0.8282\end{array}$

$\begin{array}{llll}\text { C } & 2.5584 & -5.2770 & -3.5010\end{array}$

C $2.5590-4.4830-4.8088$

O $3.5063-4.4819 \quad-5.5886$

C $2.1185-4.4577-2.2699$

$\begin{array}{llll}\text { C } & 2.9159 & -3.1645 & -2.1121\end{array}$

$\begin{array}{lllll}\text { O } & 0.7094 & -4.1996 & -2.3676\end{array}$

N $1.4092 \quad-3.7878 \quad-5.0730$

C $1.3079-2.9743 \quad-6.2547$

C $2.0415-1.6225-6.1268$

$\begin{array}{llll}\text { O } & 2.0780 & -0.9854 & -5.0669\end{array}$

C $-0.1869-2.7703-6.6051$

O $-1.0093 \quad-2.4536 \quad-5.4981$

$\begin{array}{llll}\text { N } & 2.5681 & -1.1439 & -7.2805\end{array}$

C $3.2307 \quad 0.1537 \quad-7.4099$

C $-6.0821-1.3382-7.4645$
C $-5.4110-1.6119-6.1192$

$\begin{array}{llll}\mathrm{N} & -4.9022 & -0.3768 & -5.5136\end{array}$

$\begin{array}{llll}\text { C } & -4.1760 & -0.3487 & -4.3810\end{array}$

$\begin{array}{llll}\mathrm{N} & -3.7057 & 0.8294 & -3.9372\end{array}$

N $-3.9402-1.4514 \quad-3.6905$

C $-4.2811 \quad 4.7631-5.4810$

$\begin{array}{llll}C & -3.3538 & 3.6505 & -5.9473\end{array}$

O

$\begin{array}{llll}\text { C } & -4.4798 & 4.6151 & -3.9540\end{array}$

C $-3.2546 \quad 5.0507 \quad-3.1471$

$\mathrm{N}-2.8125 \quad 4.1930 \quad-2.2140$

$\begin{array}{lllll}\mathrm{O} & -2.7510 & 6.1729 & -3.3408\end{array}$

N $-2.0313 \quad 3.9213 \quad-5.9330$

C $-1.0048 \quad 2.8746 \quad-5.9951$

C $\quad 0.0730 \quad 3.1139 \quad-4.9227$

C $\quad 0.7020 \quad 1.8354 \quad-4.3638$

$\begin{array}{llll}\text { C } & -0.3277 & 1.0444 & -3.5657\end{array}$

$\mathrm{N}-0.2134 \quad-0.3056 \quad-3.5790$

$\begin{array}{lllll}\mathrm{O} & -1.2365 & 1.6327 & -2.9654\end{array}$

C -11.7150 $-5.9954-1.0616$

C - $11.5417-4.5016-1.3636$

C - $10.1847-4.1767-1.9938$

$\begin{array}{lllll}\text { O } & -9.8573 & -4.8767 & -3.0069\end{array}$

$\begin{array}{llll}\text { O } & -9.5016 & -3.2507 & -1.4796\end{array}$

$\begin{array}{llll}\text { C } & -3.8050 & 8.4315 & 5.5642\end{array}$

$\begin{array}{llll}\text { C } & -3.4329 & 6.9461 & 5.4561\end{array}$

$\begin{array}{llll}\mathrm{N} & -3.7049 & 6.3402 & 4.1457\end{array}$

$\begin{array}{llll}\text { C } & -2.8265 & 6.1978 & 3.1339\end{array}$

$\mathrm{N}-1.6364 \quad 6.7998 \quad 3.1633$

$\begin{array}{llll}\mathrm{N} & -3.1615 & 5.4560 & 2.0717\end{array}$

C $-0.0531 \quad 12.1201-1.9933$

C $0.2724 \quad 10.8633-1.1883$

$\mathrm{N} \quad-0.5876 \quad 9.7293-1.5555$

$\begin{array}{llll}\mathrm{C} & -0.6164 & 8.5663 & -0.8697\end{array}$

$\begin{array}{llll}\mathrm{N} & 0.2222 & 8.3913 & 0.1594\end{array}$

N $-1.4795 \quad 7.5982 \quad-1.1740$

C $2.4027 \quad 6.4606-2.8801$

C $3.3131 \quad 5.2447 \quad-2.7958$

$\begin{array}{lllll}\text { O } & 2.8719 & 4.1063 & -2.6002\end{array}$

$\begin{array}{llll}\text { C } & 0.9561 & 6.1342 & -3.2333\end{array}$

N $4.6299 \quad 5.5293 \quad-2.9445$

C $5.6882 \quad 4.6145 \quad-2.5624$

C $6.3034 \quad 5.0899-1.2380$

$\begin{array}{lllll}\text { O } & 6.3026 & 6.2906 & -0.9630\end{array}$

N $6.8555 \quad 4.1121 \quad-0.4895$

$\begin{array}{llll}\text { C } & 7.5247 & 4.3872 & 0.7765\end{array}$

C $\quad 6.8608 \quad 3.7113 \quad 1.9913$

C $7.1698 \quad 2.2036 \quad 2.0695$

C $5.3532 \quad 3.9954 \quad 2.0427$

$\begin{array}{llll}\text { C } & 6.9556 & 1.6195 & 3.4679\end{array}$

C $-1.0859 \quad 1.3449 \quad 9.0524$

C $-0.7174 \quad 1.8051 \quad 7.6306$

$\begin{array}{llll}\text { C } & -1.8406 & 2.6698 & 7.0277\end{array}$

$\begin{array}{llll}\text { C } & -0.3773 & 0.6138 & 6.7252\end{array}$

$\begin{array}{llll}\text { C } & -1.4941 & 3.3160 & 5.6808\end{array}$

$\begin{array}{llll}\text { C } & -1.7418 & -2.3938 & 8.8358\end{array}$

$\begin{array}{llll}C & -1.3408 & -3.5084 & 7.8555\end{array}$

$\begin{array}{llll}\text { C } & -0.2689 & -3.0961 & 6.8687\end{array}$

$\begin{array}{llll}\text { C } & -0.5436 & -3.0018 & 5.4992\end{array}$

C $1.0318-2.8059 \quad 7.3065$ 
$\begin{array}{llll}C & 0.4534 & -2.6313 & 4.5952\end{array}$

$\begin{array}{llll}\text { C } & 2.0287 & -2.4322 & 6.4061\end{array}$

C $1.7415 \quad-2.3427 \quad 5.0422$

$\begin{array}{llll}\text { C } & -6.9441 & -2.7280 & 8.1394\end{array}$

$\begin{array}{llll}\text { C } & -5.8227 & -2.6820 & 7.0922\end{array}$

$\begin{array}{llll}\text { C } & -5.4182 & -1.2687 & 6.6294\end{array}$

$\begin{array}{llll}\text { C } & -4.1452 & -1.3200 & 5.7730\end{array}$

$\begin{array}{llll}\text { C } & -6.5392 & -0.5544 & 5.8622\end{array}$

C $-1.1355-1.4953-0.7571$

$\begin{array}{llll}\text { C } & -6.5532 & 1.6197 & 2.8062\end{array}$

$\begin{array}{llll}\text { O } & -7.0301 & 0.5399 & 2.1736\end{array}$

$\begin{array}{llll}C & -5.0624 & 1.7705 & 2.6451\end{array}$

$\begin{array}{llll}\text { O } & -7.2313 & 2.3930 & 3.4499\end{array}$

$\begin{array}{llll}\text { C } & -4.4358 & 2.7318 & 3.3511\end{array}$

$\begin{array}{llll}\text { O } & -4.5286 & 0.8781 & 1.7955\end{array}$

C $5.5094-1.0686-0.2037$

$\begin{array}{llll}\text { C } & 4.4005 & -0.2423 & -0.1839\end{array}$

$\begin{array}{llll}C & 4.5583 & 1.0245 & -0.8107\end{array}$

$\begin{array}{llll}\mathrm{N} & 3.5342 & 1.8877 & -0.9432\end{array}$

$\begin{array}{llll}\mathrm{N} & 5.7761 & 1.4122 & -1.2643\end{array}$

C $6.7937 \quad 0.5466-1.1926$

$\begin{array}{lllll}\mathrm{N} & 6.7020 & -0.6998 & -0.7061\end{array}$

$\begin{array}{llll}\text { C } & -1.4315 & -0.4415 & 0.2045\end{array}$

$\begin{array}{llll}\text { O } & -0.0188 & -2.1377 & -0.6835\end{array}$

S $1.7622 \quad 1.8109 \quad 3.2775$

C $1.7604 \quad 1.0132 \quad 1.7299$

C $8.1425 \quad 1.0022 \quad-1.6603$

$\begin{array}{llll}\text { C } & -0.5294 & -0.2716 & 1.3046\end{array}$

$\begin{array}{llll}\text { O } & -2.0030 & -1.7621 & -1.6761\end{array}$

$\begin{array}{llll}\mathrm{C} & -0.1143 & 2.5141 & 0.9913\end{array}$

$\begin{array}{llll}\text { C } & -1.0996 & -0.0124 & 2.6437\end{array}$

$\begin{array}{llll}\mathrm{N} & 2.7713 & 0.1068 & 1.6732\end{array}$

$\begin{array}{lllll}\mathrm{O} & 0.3458 & 5.9240 & 1.4264\end{array}$

$\begin{array}{lllll}\text { O } & -3.2460 & 0.8773 & -0.8362\end{array}$

$\begin{array}{llll}\text { C } & 3.5309 & -0.0002 & 2.8591\end{array}$

C $4.6604 \quad-0.9713 \quad 2.9553$

$\begin{array}{llll}\text { C } & -2.3321 & 0.4826 & 2.7864\end{array}$

$\begin{array}{llll}\text { O } & -1.2131 & 5.0996 & 0.0522\end{array}$

C $3.10620 .8346 \quad 3.8393$

C $3.5620 \quad 0.9607 \quad 5.2575$

C $-3.0981 \quad 0.9195 \quad 1.5730$

$\begin{array}{lllll}\text { O } & 0.8864 & 0.9388 & -0.5012\end{array}$

$\begin{array}{llll}\text { C } & -2.8456 & 0.0771 & 0.3050\end{array}$

$\begin{array}{llll}\text { C } & 0.6459 & 1.2050 & 0.8464\end{array}$

$\begin{array}{llll}\text { C } & 3.1183 & -0.6981 & 0.4623\end{array}$

$\begin{array}{llll}\text { C } & 0.7035 & 3.7312 & 0.5084\end{array}$

$\begin{array}{llll}\text { C } & -0.0953 & 5.0071 & 0.6668\end{array}$

H $8.1582 \quad-6.9244 \quad 0.1962$

H $6.3965-4.9126 \quad 4.6975$

H $5.3158 \quad-3.5972 \quad 4.2239$

H $7.9710-3.3468 \quad 3.6523$

H $6.8052 \quad-2.9547 \quad 2.3764$

H $8.4990 \quad-5.4504 \quad 2.6336$

H $7.9752-4.5896 \quad 1.1682$

H $4.8733 \quad-6.1784 \quad 3.3550$

$\begin{array}{llll}\text { H } & 1.9614 & -3.0687 & 1.8894\end{array}$

H $2.1663 \quad-4.1128 \quad 0.4933$

H $-1.7973-2.8514 \quad 2.9270$

H $-2.7301-2.5800 \quad 1.4614$

H $-4.1669-4.2274 \quad 2.3300$
H $-1.7152-5.3295 \quad 2.3102$

H $-2.5190 \quad-3.8324 \quad-2.7251$

H $-1.3180 \quad-4.9204-3.3177$

H $-2.8555 \quad-6.0399-4.8370$

H $-2.9695-4.2985-5.0566$

H $-4.9501-6.1788-3.6465$

H $-5.1813-5.2753 \quad-5.1447$

H $-4.7275-3.8233-2.5795$

H $-8.5543 \quad-4.6591 \quad-3.5588$

H $-7.1622 \quad-5.3730-4.4939$

H $-6.2744-2.6606-1.7135$

H $-8.0151 \quad-3.0598-2.0050$

H $-3.7550 \quad-5.8173-1.6611$

H $10.2034-3.8753-4.2928$

H $11.3851-4.9421 \quad-3.5074$

H $10.5581-4.1901-1.2406$

H $9.2501 \quad-4.8873-2.1658$

H $2.3013 \quad-5.0946 \quad-1.3907$

H $2.5299 \quad-2.6000 \quad-1.2630$

H $3.9717-3.3826 \quad-1.9304$

H $2.8293 \quad-2.5334 \quad-3.0012$

H $0.4546 \quad-3.4086-1.8249$

H $1.8885-6.1381 \quad-3.6044$

H $-0.5657-3.7225-6.9856$

H $-0.2708-2.0253 \quad-7.4102$

H $-0.8202-1.5571 \quad-5.1750$

H $1.7745 \quad-3.5233 \quad-7.0808$

$\begin{array}{llll}\text { H } & 3.3812 & 0.5517 & -6.4063\end{array}$

H $2.6198 \quad 0.8527 \quad-7.9910$

H $-6.9289-0.6557-7.3492$

H $-5.3756-0.9038-8.1787$

H $-6.1284-2.0831-5.4380$

H $-4.5748-2.3107-6.2530$

H $-4.95320 .4907 \quad-6.0339$

$\begin{array}{llll}\mathrm{H} & -2.9775 & 0.8343 & -3.2176\end{array}$

H $-3.6479 \quad 1.5583 \quad-4.6437$

H $-3.3572-1.4184 \quad-2.8499$

H $-4.4441-2.3124 \quad-3.8781$

H $-4.7735 \quad 3.5894 \quad-3.7122$

$\begin{array}{llll}\mathrm{H} & -5.2988 & 5.2717 & -3.6413\end{array}$

H $-3.1001 \quad 3.2244 \quad-2.1771$

H $-2.0345 \quad 4.4467 \quad-1.6071$

$\mathrm{H} \quad-3.8763 \quad 5.7561 \quad-5.6911$

H $0.8626 \quad 3.7584 \quad-5.3206$

H $-0.3905 \quad 3.6340-4.0820$

H $1.5098 \quad 2.1190 \quad-3.6782$

H $1.1522 \quad 1.2051 \quad-5.1338$

H $-0.8268-0.8571-2.9700$

H $\quad 0.6601-0.7204 \quad-3.9104$

H $-0.5649 \quad 2.8376 \quad-6.9991$

H $-1.7490 \quad 4.8406 \quad-5.6188$

H -12.7109 $-6.2053-0.6586$

H $-11.5742-6.5790-1.9744$

H $-12.3160-4.1857-2.0749$

H -11.6558 $-3.8951 \quad-0.4613$

$\begin{array}{llll}\mathrm{H} & -4.8701 & 8.5819 & 5.3695\end{array}$

H $-3.2417 \quad 9.0408 \quad 4.8506$

H $-3.9942 \quad 6.3633 \quad 6.1911$

H $-2.3763 \quad 6.7806 \quad 5.6877$

H $-4.6385 \quad 5.9938 \quad 3.9770$
$\begin{array}{llll}\mathrm{H} & -1.4251 & 7.4650 & 3.8879\end{array}$

$\begin{array}{llll}\mathrm{H} & -0.8872 & 6.5156 & 2.5032\end{array}$

H $-3.8646 \quad 4.7324 \quad 2.1764$

H $-2.5045 \quad 5.3678 \quad 1.2814$

H $-1.082912 .4454-1.8187$

H $\quad 0.6143 \quad 12.9285 \quad-1.6884$

H $\quad 0.1416 \quad 11.0803 \quad-0.1200$

H $1.3207 \quad 10.5817 \quad-1.3475$

$\mathrm{H}-1.3162 \quad 9.8811 \quad-2.2374$

H $-1.4119 \quad 6.7103 \quad-0.6517$

H $-1.9242 \quad 7.4888-2.0865$

$\begin{array}{llll}\mathrm{H} & 0.2838 & 7.4760 & 0.6394\end{array}$

$\mathrm{H} \quad 0.8708 \quad 9.1154 \quad 0.4155$

$\begin{array}{lllll}\mathrm{H} & 0.5291 & 5.4181 & -2.5287\end{array}$

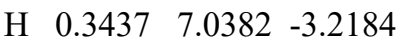

H $0.8868 \quad 5.6992 \quad-4.2311$

H $2.4451 \quad 6.9446-1.8935$

H $5.3073 \quad 3.5964-2.5084$

H $6.4760 \quad 4.6271 \quad-3.3249$

$\begin{array}{llll}\mathrm{H} & 7.3301 & 4.1794 & 2.8690\end{array}$

H $4.9131 \quad 3.6407 \quad 2.9794$

H $5.1520 \quad 5.0681 \quad 1.9606$

H $4.8304 \quad 3.4945 \quad 1.2210$

$\begin{array}{llll}\mathrm{H} & 6.5508 & 1.6614 & 1.3442\end{array}$

$\begin{array}{llll}\mathrm{H} & 8.2127 & 2.0353 & 1.7727\end{array}$

$\begin{array}{llll}\text { H } & 5.9090 & 1.6949 & 3.7757\end{array}$

H $7.2411 \quad 0.5642 \quad 3.5070$

H $7.5607 \quad 2.1527 \quad 4.2089$

H $7.5055 \quad 5.4725 \quad 0.8932$

$\begin{array}{llll}\mathrm{H} & 0.1802 & 2.4386 & 7.7078\end{array}$

H $\quad 0.3864 \quad-0.0285 \quad 7.1717$

$\begin{array}{llll}\mathrm{H} & -1.2583 & -0.0130 & 6.5481\end{array}$

$\begin{array}{llll}\mathrm{H} & -0.0049 & 0.9391 & 5.7486\end{array}$

$\begin{array}{llll}\mathrm{H} & -2.1034 & 3.4582 & 7.7453\end{array}$

H $-2.7401 \quad 2.0490 \quad 6.9124$

H $-2.3087 \quad 3.9635 \quad 5.3363$

H $-0.5884 \quad 3.9298 \quad 5.7568$

H $-1.3186 \quad 2.5676 \quad 4.9014$

$\begin{array}{llll}\mathrm{H} & -1.2637 & 2.2002 & 9.7124\end{array}$

H $-0.9875 \quad-4.3754 \quad 8.4273$

H $-2.2253 \quad-3.8435 \quad 7.3037$

H $-1.5393 \quad-3.2412 \quad 5.1366$

H $\quad 1.2675 \quad-2.8864 \quad 8.3644$

H $\quad 0.2239 \quad-2.5810 \quad 3.5370$

H $3.0325-2.2261 \quad 6.7672$

H $2.5153 \quad-2.0658 \quad 4.3354$

H $-2.1399 \quad-1.5272 \quad 8.3011$

H $-4.9331 \quad-3.1750 \quad 7.5066$

H $-6.1111-3.2721 \quad 6.2118$

H $-5.1964-0.6782 \quad 7.5322$

H $-4.2966-1.9047 \quad 4.8582$

$\begin{array}{llll}\mathrm{H} & -3.3173 & -1.7780 & 6.3228\end{array}$

H $-3.8362-0.3092 \quad 5.4803$

$\begin{array}{llll}\mathrm{H} & -7.4660 & -0.4986 & 6.4403\end{array}$

$\begin{array}{llll}\mathrm{H} & -6.2495 & 0.4717 & 5.6091\end{array}$

H $-6.7573-1.0792 \quad 4.9241$

H $-7.8843 \quad-2.3265 \quad 7.7490$

H $-5.0415 \quad 3.3268 \quad 4.0220$

$\begin{array}{llll}\mathrm{H} & -3.3624 & 2.8448 & 3.3724\end{array}$

$\begin{array}{llll}\text { H } & 5.4482 & -2.0731 & 0.2044\end{array}$ 


\begin{abstract}
H $\quad 2.5837 \quad 1.5373-0.8694$
H $0.0388 \quad 0.5357 \quad-0.8282$

H $\quad 8.0618 \quad 1.9138 \quad-2.2531$

$\begin{array}{lllll}\text { H } & 8.7813 & 1.2049 & -0.7921\end{array}$

$\begin{array}{llll}\text { H } & 8.6176 & 0.2033 & -2.2360\end{array}$

H $\quad 0.2521 \quad-1.0276 \quad 1.3085$

$\begin{array}{llll}\mathrm{H} & -0.4508 & 2.6587 & 2.0240\end{array}$

$\begin{array}{llll}\mathrm{H} & -1.0042 & 2.4261 & 0.3635\end{array}$

$\begin{array}{llll}\mathrm{H} & -0.5212 & -0.2890 & 3.5185\end{array}$

$\begin{array}{llll}\mathrm{H} & -4.2144 & 0.8800 & -0.8170\end{array}$

H $4.9437 \quad-1.1037 \quad 3.9991$

H $4.4053 \quad-1.9476 \quad 2.5416$

H $5.5368 \quad-0.6130 \quad 2.4115$

$\begin{array}{llll}\mathrm{H} & -2.7819 & 0.6075 & 3.7639\end{array}$

H $2.8149 \quad 0.5413 \quad 5.9405$

H $4.5012 \quad 0.4279 \quad 5.4120$

H $-2.8498 \quad 1.9601 \quad 1.3226$

$\begin{array}{llll}\mathrm{H} & -3.5357 & -0.7769 & 0.3511\end{array}$

H $3.2438 \quad-1.7315 \quad 0.7812$

H $2.2581 \quad-0.6520 \quad-0.2009$

H $1.6380 \quad 3.8321 \quad 1.0633$

H $0.9455 \quad 3.5929 \quad-0.5509$

H -10.9766 $-6.3358-0.3277$

$\begin{array}{llll}\mathrm{H} & -3.5871 & 8.7924 & 6.5729\end{array}$

H $-1.9958 \quad 0.7351 \quad 9.0408$

$\begin{array}{llll}\mathrm{H} & -0.2870 & 0.7381 & 9.4894\end{array}$

H $-7.1354-3.7559 \quad 8.4616$

H $-6.6764-2.14369 .0272$

H $-2.5099-2.7470 \quad 9.5302$

$\begin{array}{llll}\mathrm{H} & -0.8848 & -2.0529 & 9.4245\end{array}$

H $11.5272-3.1791-3.3640$

H $7.5380-8.5692 \quad 0.5031$

H $8.4829-7.7082 \quad 1.7451$

$\begin{array}{llll}\mathrm{H} & -6.4581 & -2.2753 & -7.8803\end{array}$

H $3.6307 \quad 2.6967 \quad-1.5528$

H $2.8257 \quad 7.1797 \quad-3.5906$

H $7.8665-1.9462-0.7975$

$\begin{array}{llll}\text { O } & -4.4284 & -2.7880 & -0.9800\end{array}$

H $-3.5248-2.4167-1.1067$

$\begin{array}{llll}\mathrm{H} & -4.3357 & -3.4988 & -0.3166\end{array}$

H $3.72692 .0070 \quad 5.5332$

$\begin{array}{llll}\mathrm{H} & 8.5741 & 4.0741 & 0.7100\end{array}$

H $\quad 0.0867 \quad 11.9517 \quad-3.0651$

$\begin{array}{llll}\mathrm{H} & -7.9854 & 0.5044 & 2.3526\end{array}$

H $2.5993 \quad-1.7699 \quad-8.0717$

$\begin{array}{lllll}\text { H } & 4.2008 & 0.0334 & -7.8979\end{array}$

H $3.5739 \quad-5.6486 \quad-3.3558$

H $0.7190 \quad-3.6895 \quad-4.3329$

H $2.7127 \quad-5.5982 \quad 2.8590$

$\begin{array}{llll}\mathrm{H} & -0.2227 & -3.4220 & 0.5544\end{array}$

H $-0.9755 \quad-5.2651 \quad-0.7952$

H $4.8988 \quad 6.5009 \quad-2.8386$

H $6.6260 \quad 3.1534 \quad-0.7520$

H $-2.4195 \quad-6.8428-2.1882$

$\begin{array}{lllll}\mathrm{H} & -5.2356 & 4.6451 & -5.9948\end{array}$

H $-1.5242 \quad 1.9278 \quad-5.8381$
\end{abstract}

\section{Int4}

C $7.7455 \quad-7.4927 \quad 1.5944$
C $\quad 6.4017 \quad-6.9565 \quad 2.0663$

$\begin{array}{llll}\text { O } & 5.3874 & -7.6466 & 2.0937\end{array}$

$\begin{array}{llll}\mathrm{N} & 6.3827 & -5.6555 & 2.5033\end{array}$

C $5.2200 \quad-5.1645 \quad 3.2351$

C $4.2096-4.4454 \quad 2.3397$

$\begin{array}{llll}\text { O } & 4.5651 & -3.5910 & 1.5125\end{array}$

C $5.8475-4.1469 \quad 4.2139$

C $7.0206-3.5602 \quad 3.4126$

$\begin{array}{llll}\text { C } & 7.5381 & -4.7485 & 2.5824\end{array}$

$\begin{array}{llll}\text { N } & 2.9090 & -4.6826 & 2.5763\end{array}$

C 1.8888 -3.9281 1.8893

$\begin{array}{llll}\text { C } & 0.5177 & -4.4820 & 2.2519\end{array}$

$\begin{array}{lllll}\mathrm{O} & 0.3872 & -5.3163 & 3.1431\end{array}$

$\begin{array}{llll}\mathrm{N} & -0.4943 & -3.9420 & 1.5288\end{array}$

$\begin{array}{llll}\text { C } & -1.8782 & -4.2024 & 1.8769\end{array}$

$\begin{array}{llll}\text { C } & -2.6882 & -4.7565 & 0.7070\end{array}$

$\begin{array}{llll}\text { O } & -3.9303 & -4.6372 & 0.7137\end{array}$

C $-2.5671-2.9461 \quad 2.4502$

$\begin{array}{llll}\mathrm{O} & -3.7875 & -3.2421 & 3.0893\end{array}$

$\begin{array}{llll}\mathrm{N} & -2.0167 & -5.3844 & -0.2699\end{array}$

C $-2.6812-5.9410-1.4420$

$\begin{array}{llll}\text { C } & -2.3914 & -5.1035 & -2.6923\end{array}$

C $-3.1544 \quad-5.5655 \quad-3.9389$

C $-4.6771 \quad-5.6462 \quad-3.7917$

N $-5.2585 \quad-4.3733 \quad-3.3841$

$\begin{array}{llll}\text { C } & -6.6069 & -4.2538 & -3.1562\end{array}$

$\mathrm{N}-7.4744 \quad-5.1442 \quad-3.6243$

$\begin{array}{llll}\mathrm{N} & -7.0440 & -3.1747 & -2.5067\end{array}$

$\begin{array}{llll}\text { C } & 10.8398 & -4.2437 & -3.0068\end{array}$

C $9.9255-4.2112-1.7850$

C $9.1061-2.9395-1.7019$

$\begin{array}{llll}\text { O } & 9.1168 & -2.0742 & -2.5616\end{array}$

$\begin{array}{llll}\text { O } & 8.3704 & -2.8703 & -0.5878\end{array}$

$\begin{array}{llll}\text { C } & 2.5790 & -5.5197 & -3.0353\end{array}$

C 2.6098 -4.8680 -4.4148

O $3.5713-4.9432 \quad-5.1726$

C $2.1306-4.5706-1.9021$

C $2.9576-3.2877-1.8574$

O $0.7314 \quad-4.2878 \quad-2.0575$

N $1.4631 \quad-4.2074 \quad-4.7709$

C $1.4113-3.4916 \quad-6.0144$

C $2.1673-2.1451-5.9652$

O $2.3131-1.4948 \quad-4.9244$

$\begin{array}{llll}\text { C } & -0.0597 & -3.2979 & -6.4422\end{array}$

$\begin{array}{lllll}\mathrm{O} & -0.9095 & -2.8540 & -5.3980\end{array}$

N $2.5943 \quad-1.6850 \quad-7.1649$

C $3.2600-0.3988-7.3406$

$\begin{array}{llll}\text { C } & -6.0517 & -1.8981 & -7.3105\end{array}$

$\begin{array}{llll}\text { C } & -4.8557 & -2.1018 & -6.3725\end{array}$

$\begin{array}{llll}\mathrm{N} & -4.4890 & -0.8545 & -5.6907\end{array}$

$\begin{array}{llll}\text { C } & -3.7869 & -0.7930 & -4.5488\end{array}$

$\begin{array}{llll}\mathrm{N} & -3.6977 & 0.3812 & -3.8853\end{array}$

$\mathrm{N}-3.2222 \quad-1.8733-4.0220$

$\begin{array}{llll}C & -4.2604 & 4.3371 & -5.7876\end{array}$

$\begin{array}{llll}C & -3.3337 & 3.1761 & -6.1130\end{array}$

$\begin{array}{lllll}\text { O } & -3.7796 & 2.0246 & -6.2378\end{array}$

C $-4.4915 \quad 4.3525-4.2566$

C $-3.2453 \quad 4.7697 \quad-3.4717$

N $-2.8859 \quad 3.9944 \quad-2.4368$

O $-2.6390 \quad 5.8115 \quad-3.7863$
N $-2.0128 \quad 3.4573 \quad-6.1527$

C $-0.9813 \quad 2.4176-6.1478$

C $\quad 0.1143 \quad 2.7146 \quad-5.1118$

C $0.7868 \quad 1.4559 \quad-4.5577$

$\begin{array}{llll}\text { C } & -0.1941 & 0.6654 & -3.6985\end{array}$

N $0.0156 \quad-0.6709 \quad-3.5888$

$\begin{array}{lllll}\mathrm{O} & -1.1454 & 1.2392 & -3.1559\end{array}$

C $-11.7015-6.0636-0.5914$

C $-11.4843-4.6126-1.0313$

C - $10.1546-4.3939-1.7629$

$\begin{array}{lllll}\text { O } & -9.8119 & -5.3025 & -2.5849\end{array}$

$\begin{array}{llll}\text { O } & -9.5157 & -3.3366 & -1.5117\end{array}$

C $\quad-3.8201 \quad 8.8285 \quad 4.9509$

C $-3.4420 \quad 7.3399 \quad 4.9582$

$\begin{array}{llll}\mathrm{N} & -3.7158 & 6.6290 & 3.7015\end{array}$

$\begin{array}{llll}\text { C } & -2.8376 & 6.4044 & 2.7044\end{array}$

$\begin{array}{llll}\mathrm{N} & -1.6549 & 7.0231 & 2.6774\end{array}$

$\mathrm{N}-3.1629 \quad 5.5656 \quad 1.7153$

C $-0.0473 \quad 11.9394 \quad-2.8521$

C $\quad 0.2454 \quad 10.7583 \quad-1.9283$

$\begin{array}{llll}\mathrm{N} & -0.5948 & 9.5912 & -2.2335\end{array}$

$\begin{array}{llll}\text { C } & -0.6249 & 8.4778 & -1.4691\end{array}$

$\begin{array}{lllll}\mathrm{N} & 0.1974 & 8.3800 & -0.4177\end{array}$

N $-1.4737 \quad 7.4820 \quad-1.7216$

$\begin{array}{lllll}\text { C } & 2.4145 & 6.2312 & -3.3021\end{array}$

C $3.3249 \quad 5.0258 \quad-3.1274$

$\begin{array}{lllll}\text { O } & 2.8835 & 3.9032 & -2.8551\end{array}$

C $0.9712 \quad 5.8809-3.6453$

$\begin{array}{llll}\mathrm{N} & 4.6426 & 5.3003 & -3.2894\end{array}$

$\begin{array}{llll}\text { C } & 5.6991 & 4.4168 & -2.8358\end{array}$

C $6.3064 \quad 4.9887-1.5465$

$\begin{array}{lllll}\text { O } & 6.2961 & 6.2055 & -1.3568\end{array}$

$\begin{array}{lllll}\mathrm{N} & 6.8645 & 4.0712 & -0.7284\end{array}$

$\begin{array}{llll}\text { C } & 7.5266 & 4.4437 & 0.5159\end{array}$

C $6.8668 \quad 3.8512 \quad 1.7754$

$\begin{array}{llll}\text { C } & 7.1913 & 2.3565 & 1.9588\end{array}$

C $\begin{array}{llll}\text { C.3562 } & 4.1237 & 1.8026\end{array}$

C $\quad 6.9508 \quad 1.8605 \quad 3.3863$

C $\begin{array}{llll}-1.1076 & 2.0274 & 8.9719\end{array}$

$\begin{array}{llll}\text { C } & -0.7229 & 2.3392 & 7.5137\end{array}$

$\begin{array}{llll}\text { C } & -1.8199 & 3.1748 & 6.8268\end{array}$

$\begin{array}{llll}\text { C } & -0.4174 & 1.0570 & 6.7292\end{array}$

$\begin{array}{llll}\text { C } & -1.4747 & 3.6401 & 5.4068\end{array}$

C $-1.7605-1.7175 \quad 9.0360$

C $-1.3955-2.8937 \quad 8.1150$

C $-0.3097-2.5693 \quad 7.1105$

$\begin{array}{llll}\text { C } & -0.5896 & -2.4828 & 5.7417\end{array}$

C $1.0106-2.3536 \quad 7.5322$

C $0.4201 \quad-2.1964 \quad 4.8211$

C $2.0219-2.0646 \quad 6.6164$

C $1.7288-1.9867 \quad 5.2529$

$\begin{array}{llll}\text { C } & -6.9605 & -2.1075 & 8.3511\end{array}$

C $-5.8121 \quad-2.0599 \quad 7.3330$

$\begin{array}{llll}\text { C } & -5.5625 & -0.6802 & 6.6938\end{array}$

C $-4.2862-0.6955 \quad 5.8394$

C $-6.7499-0.1977 \quad 5.8493$

C $-1.1194-1.4398-0.6791$

$\begin{array}{llll}\text { C } & -6.5900 & 1.7921 & 2.6359\end{array}$

$\begin{array}{lllll}\text { O } & -7.0567 & 0.6796 & 2.0578\end{array}$

C $-5.0992 \quad 1.9462 \quad 2.4717$ 
$\begin{array}{llll}\text { O } & -7.2753 & 2.5907 & 3.2414\end{array}$

$\begin{array}{llll}\text { C } & -4.4835 & 2.9466 & 3.1354\end{array}$

$\begin{array}{llll}\text { O } & -4.5582 & 1.0202 & 1.6695\end{array}$

C $5.4461-1.0938-0.0964$

$\begin{array}{llll}\text { C } & 4.3486 & -0.2515 & -0.1272\end{array}$

$\begin{array}{llll}C & 4.5246 & 0.9748 & -0.8262\end{array}$

$\begin{array}{lllll}\text { N } & 3.5151 & 1.8440 & -1.0098\end{array}$

$\begin{array}{llll}\mathrm{N} & 5.7487 & 1.3173 & -1.2995\end{array}$

$\begin{array}{llll}\text { C } & 6.7532 & 0.4428 & -1.1769\end{array}$

$\begin{array}{llll}\mathrm{N} & 6.6436 & -0.7719 & -0.6174\end{array}$

$\begin{array}{llll}\mathrm{C} & -1.4247 & -0.3133 & 0.1568\end{array}$

$\begin{array}{lllll}\text { O } & 0.0016 & -2.0886 & -0.5350\end{array}$

S $1.6984 \quad 2.0131 \quad 3.1865$

$\begin{array}{llll}\text { C } & 1.7127 & 1.1354 & 1.7011\end{array}$

$\begin{array}{llll}\text { C } & 8.1087 & 0.8529 & -1.6665\end{array}$

$\begin{array}{llll}\text { C } & -0.4850 & -0.0222 & 1.2631\end{array}$

$\begin{array}{llll}\text { O } & -1.9604 & -1.7986 & -1.6037\end{array}$

$\begin{array}{llll}C & -0.1674 & 2.5861 & 0.8584\end{array}$

$\begin{array}{llll}\text { C } & -1.1274 & 0.2331 & 2.5928\end{array}$

$\begin{array}{llll}\mathrm{N} & 2.7204 & 0.2409 & 1.6949\end{array}$

$\begin{array}{lllll}\mathrm{O} & 0.3486 & 6.0150 & 1.0207\end{array}$

$\begin{array}{lllll}\text { O } & -3.2563 & 1.0061 & -0.9434\end{array}$

C $3.4875 \quad 0.2094 \quad 2.8783$

C $4.6250 \quad-0.7429 \quad 3.0365$

$\begin{array}{llll}\text { C } & -2.3779 & 0.6850 & 2.6811\end{array}$

$\begin{array}{lllll}\text { O } & -1.2115 & 5.0883 & -0.2859\end{array}$

C $3.0525 \quad 1.1036 \quad 3.8060$

$\begin{array}{llll}\text { C } & 3.5074 & 1.3129 & 5.2155\end{array}$

$\begin{array}{llll}\text { C } & -3.1317 & 1.0753 & 1.4432\end{array}$

$\begin{array}{lllll}\text { O } & 0.8731 & 0.9603 & -0.5389\end{array}$

$\begin{array}{llll}\text { C } & -2.8425 & 0.2062 & 0.2032\end{array}$

$\begin{array}{llll}\text { C } & 0.5368 & 1.2226 & 0.8044\end{array}$

$\begin{array}{llll}\text { C } & 3.0587 & -0.6535 & 0.5368\end{array}$

$\begin{array}{llll}C & 0.6844 & 3.7429 & 0.3072\end{array}$

$\begin{array}{llll}\text { C } & -0.1006 & 5.0391 & 0.3439\end{array}$

H $8.1962 \quad-6.8476 \quad 0.8340$

H $6.2112 \quad-4.6882 \quad 5.0927$

H $5.1305 \quad-3.3959 \quad 4.5568$

H 7.7998 -3.1376 4.0518

H $6.6634 \quad-2.7787 \quad 2.7402$

H $8.3789 \quad-5.2448 \quad 3.0821$

H $7.8652-4.4305 \quad 1.5876$

H $4.7356-6.00413 .7383$

H $1.9162 \quad-2.8733 \quad 2.1922$

H $2.0476 \quad-3.9459 \quad 0.8092$

H $-1.9087-2.5130 \quad 3.2033$

H $-2.6792-2.2012 \quad 1.6512$

H $-4.3201 \quad-3.6992 \quad 2.4144$

H $-1.8721 \quad-4.9676 \quad 2.6605$

H $-2.6115-4.0617-2.4488$

H $-1.3179-5.1265-2.8971$

H $-2.8079-6.5641-4.2325$

H $-2.8987-4.9017-4.7739$

H $-4.9384-6.4465-3.0824$

H $-5.0995-5.9350-4.7615$

H $-4.7167-3.7993-2.7337$

H $-8.5030-5.1637 \quad-3.2149$

H $-7.1064-5.9921-4.0242$

$\begin{array}{llll}\mathrm{H} & -6.3391 & -2.6392 & -2.0103\end{array}$

H $-8.0439-3.1890 \quad-2.1463$
H $-3.7461-5.9553-1.2061$

H $10.2584-4.1502-3.9268$

H $11.4025-5.1806-3.0430$

H $10.4921-4.3053-0.8508$

H $9.2217 \quad-5.0532-1.7888$

H $2.2793 \quad-5.1211 \quad-0.9603$

H $2.5679-2.6326 \quad-1.0779$

H $4.0042-3.5101-1.6322$

H $2.9034-2.7497 \quad-2.8077$

H $0.4753-3.4643 \quad-1.5613$

H $1.8998-6.3795 \quad-3.0605$

H $-0.4531-4.2709-6.7460$

H $-0.0997-2.6251-7.3112$

H $-0.6351-1.9772 \quad-5.0722$

H 1.9048 -4.1063 -6.7763

H $3.4580 \quad 0.0097 \quad-6.3500$

H $2.6301 \quad 0.2983 \quad-7.9036$

H $-6.9315-1.5718-6.7503$

H $-5.8292-1.1557-8.0842$

H $-5.1053-2.8371 \quad-5.6001$

H $-3.9943 \quad-2.4858 \quad-6.9371$

H $-4.6763 \quad 0.0315-6.1462$

$\mathrm{H}-2.8642 \quad 0.5090 \quad-3.2990$

H $-3.9223 \quad 1.1876 \quad-4.4599$

H $-2.7085-1.7954 \quad-3.1314$

H $-2.9994 \quad-2.6596 \quad-4.6127$

H $-4.8679 \quad 3.3801 \quad-3.9247$

H $-5.2644 \quad 5.0928-4.0238$

H $-3.2964 \quad 3.0920-2.2307$

H $-2.1034 \quad 4.2672-1.8446$

$\begin{array}{llll}\mathrm{H} & -3.8409 & 5.2987 & -6.0934\end{array}$

H $\quad 0.8755 \quad 3.3716 \quad-5.5432$

H $-0.3406 \quad 3.2361 \quad-4.2674$

H $1.6261 \quad 1.7557 \quad-3.9182$

H $\quad 1.2040 \quad 0.8152 \quad-5.3395$

H $-0.5331-1.1772-2.8910$

H $\quad 0.9198-1.0571 \quad-3.8689$

H $\quad-0.5537 \quad 2.3085 \quad-7.1517$

H $-1.73624 .4050 \quad-5.9314$

H -12.6867 $-6.1982-0.1327$

H -11.6171 $-6.7297 \quad-1.4526$

H $-12.2847 \quad-4.3187-1.7238$

H -11.5264 $-3.9223-0.1842$

H $-4.8867 \quad 8.9594 \quad 4.7505$

H $-3.2627 \quad 9.3820 \quad 4.1886$

$\begin{array}{llll}\mathrm{H} & -3.9981 & 6.8155 & 5.7397\end{array}$

H $-2.3835 \quad 7.1994 \quad 5.1979$

$\begin{array}{llll}\mathrm{H} & -4.6408 & 6.2434 & 3.5753\end{array}$

H $-1.4535 \quad 7.7532 \quad 3.3395$

H $-0.9012 \quad 6.68992 .0480$

H $-3.8660 \quad 4.8512 \quad 1.8768$

H $-2.5133 \quad 5.4260 \quad 0.9273$

H $-1.083912 .2741 \quad-2.7501$

$\begin{array}{llll}\mathrm{H} & 0.6052 & 12.7746 & -2.5898\end{array}$

H $\quad 0.0712 \quad 11.0656-0.8884$

H $1.2994 \quad 10.4687 \quad-2.0204$

H $-1.3256 \quad 9.6960 \quad-2.9219$

H $-1.4110 \quad 6.6348 \quad-1.1353$

H $-1.8894 \quad 7.3038-2.6356$

H $\quad 0.2629 \quad 7.4978 \quad 0.1220$
H $\quad 0.8188 \quad 9.1354 \quad-0.1853$

H $\quad 0.5295 \quad 5.2274-2.8910$

H $0.3635 \quad 6.7854 \quad-3.7126$

H $0.9113 \quad 5.3664 \quad-4.6052$

H $2.4483 \quad 6.7821 \quad-2.3507$

H $5.3187 \quad 3.4047 \quad-2.7098$

H $6.4916 \quad 4.3749 \quad-3.5922$

H $7.3293 \quad 4.3846 \quad 2.6186$

$\mathrm{H} \quad 4.9156 \quad 3.8245 \quad 2.7584$

H $5.1457 \quad 5.1871 \quad 1.6528$

H $4.8427 \quad 3.5679 \quad 1.0109$

$\begin{array}{llll}\mathrm{H} & 6.5964 & 1.7618 & 1.2547\end{array}$

H $\quad 8.2430 \quad 2.1845 \quad 1.6978$

H $5.8971 \quad 1.9469 \quad 3.6656$

H $7.2437 \quad 0.8127 \quad 3.4997$

H $7.5343 \quad 2.4459 \quad 4.1048$

H $7.4980 \quad 5.5343 \quad 0.5537$

$\begin{array}{llll}\mathrm{H} & 0.1930 & 2.9508 & 7.5344\end{array}$

$\begin{array}{llll}\mathrm{H} & 0.3210 & 0.4347 & 7.2417\end{array}$

$\begin{array}{llll}\mathrm{H} & -1.3190 & 0.4487 & 6.6033\end{array}$

$\begin{array}{llll}\mathrm{H} & -0.0294 & 1.2721 & 5.7282\end{array}$

H $-2.0353 \quad 4.0533 \quad 7.4493$

H $-2.7465 \quad 2.5849 \quad 6.7984$

H $-2.2541 \quad 4.3019 \quad 5.0112$

H $-0.5280 \quad 4.1941 \quad 5.3890$

$\mathrm{H}-1.3784 \quad 2.7980 \quad 4.7143$

H $-1.2623 \quad 2.9467 \quad 9.5463$

H $-1.0691 \quad-3.7405 \quad 8.7311$

H $-2.2910 \quad-3.2277 \quad 7.5806$

H $-1.6027 \quad-2.6594 \quad 5.3926$

H $\quad 1.2498 \quad-2.4285 \quad 8.5898$

H $\quad 0.1845 \quad-2.1554 \quad 3.7632$

H $3.0405 \quad-1.9190 \quad 6.9654$

H $2.5146 \quad-1.7842 \quad 4.5335$

H $-2.1620-0.8806 \quad 8.4591$

H $-4.8863 \quad-2.3805 \quad 7.8300$

$\begin{array}{llll}\mathrm{H} & -5.9945 & -2.7910 & 6.5338\end{array}$

H $\quad-5.4144 \quad 0.0402 \quad 7.5133$

H $-4.3742-1.3948 \quad 4.9993$

H $-3.4117 \quad-0.9903 \quad 6.4294$

$\begin{array}{llll}\mathrm{H} & -4.0898 & 0.3022 & 5.4282\end{array}$

H $-7.6782-0.1616 \quad 6.4265$

H $-6.5700 \quad 0.8089 \quad 5.4565$

H $-6.9089-0.8665 \quad 4.9949$

H $-7.9266-1.8899 \quad 7.8860$

H $-5.0972 \quad 3.5673 \quad 3.7753$

H $-3.4105 \quad 3.0631 \quad 3.1620$

H $5.3702 \quad-2.0716 \quad 0.3699$

H $2.5570 \quad 1.5157 \quad-0.9166$

$\begin{array}{lllll}\mathrm{H} & 0.0324 & 0.5524 & -0.8959\end{array}$

H $\quad 8.0385 \quad 1.7178 \quad-2.3269$

H $\quad 8.7411 \quad 1.1186-0.8107$

H $8.5848 \quad 0.0104-2.1752$

$\begin{array}{llll}\mathrm{H} & 0.2057 & -0.8644 & 1.3446\end{array}$

H $-0.5134 \quad 2.8059 \quad 1.8744$

$\begin{array}{llll}\mathrm{H} & -1.0527 & 2.4831 & 0.2281\end{array}$

$\begin{array}{llll}\mathrm{H} & -0.5837 & -0.0234 & 3.4966\end{array}$

H $-3.9681 \quad 0.5173-1.3761$

H $4.9126 \quad-0.7974 \quad 4.0862$

H $4.3751 \quad-1.7485 \quad 2.6951$ 
H $5.4948 \quad-0.4177 \quad 2.4630$

$\begin{array}{llll}\mathrm{H} & -2.8732 & 0.7916 & 3.6387\end{array}$

H $2.8191 \quad 0.8221 \quad 5.9126$

H $4.5043 \quad 0.8972 \quad 5.3675$

H $-2.89912 .1134 \quad 1.1685$

$\begin{array}{llll}\mathrm{H} & -3.5376 & -0.6409 & 0.2498\end{array}$

$\begin{array}{llll}\text { H } & 3.1732 & -1.6604 & 0.9345\end{array}$

H $2.1966-0.6433-0.1262$

$\begin{array}{llll}\mathrm{H} & 1.6075 & 3.8806 & 0.8736\end{array}$

H $0.9502 \quad 3.5233-0.7324$

H $-10.9453 \quad-6.3688 \quad 0.1397$

H $-3.5983 \quad 9.2682 \quad 5.9269$

H $-2.0351 \quad 1.4460 \quad 9.0147$

H $-0.3273 \quad 1.4432 \quad 9.4690$

H $-7.0335-3.0978 \quad 8.8105$

H $-6.8055-1.3772 \quad 9.1537$

H $-2.5163-2.0192 \quad 9.7671$

H $-0.8853-1.3533 \quad 9.5829$

H $11.5502-3.4135$ - 2.9838

H $7.5869 \quad-8.4890 \quad 1.1845$

H $8.4477 \quad-7.5563 \quad 2.4328$

H $-6.2897-2.8419-7.8062$

H $3.6264 \quad 2.6132 \quad-1.6669$

H $2.8436 \quad 6.9006-4.0563$

H $7.8020 \quad-2.0371-0.6230$

O $-4.4813-2.6142-1.1759$

H $-3.5509-2.2895-1.2412$

$\begin{array}{llll}\mathrm{H} & -4.4686 & -3.2817 & -0.4646\end{array}$

$\begin{array}{llll}\mathrm{H} & 3.5500 & 2.3763 & 5.4677\end{array}$

$\begin{array}{lllll}\text { H } & 8.5788 & 4.1358 & 0.4745\end{array}$

H $\quad 0.1382 \quad 11.6796 \quad-3.8983$

$\begin{array}{llll}\mathrm{H} & -8.0123 & 0.6456 & 2.2348\end{array}$

H $2.5463 \quad-2.3099 \quad-7.9562$

H $4.2074-0.5301-7.8696$

H $3.5867 \quad-5.8854-2.8320$

H $0.7752 \quad-4.0171-4.0456$

H $2.5734 \quad-5.3970 \quad 3.2138$

$\begin{array}{llll}\mathrm{H} & -0.2828 & -3.2347 & 0.8010\end{array}$

$\begin{array}{llll}\mathrm{H} & -1.0082 & -5.2911 & -0.2907\end{array}$

$\begin{array}{llll}\text { H } & 4.9098 & 6.2774 & -3.2501\end{array}$

$\begin{array}{lllll}\text { H } & 6.6399 & 3.0951 & -0.9187\end{array}$

H $-2.3511-6.9773-1.5793$

H $\quad-5.2067 \quad 4.1726-6.3042$

H $-1.5018 \quad 1.4869-5.9140$

\section{TS5}

$\begin{array}{llll}C & 7.9026 & -7.2520 & 1.9578\end{array}$

$\begin{array}{llll}\text { C } & 6.5201 & -6.7682 & 2.3714\end{array}$

$\begin{array}{llll}\text { O } & 5.5443 & -7.5108 & 2.4100\end{array}$

$\begin{array}{llll}\mathrm{N} & 6.4218 & -5.4500 & 2.7426\end{array}$

C $5.2225 \quad-4.9862 \quad 3.4297$

$\begin{array}{llll}\text { C } & 4.2121 & -4.3161 & 2.4960\end{array}$

$\begin{array}{llll}\text { O } & 4.5658 & -3.5143 & 1.6185\end{array}$

C $5.7862-3.9256 \quad 4.4030$

C $6.9531 \quad-3.3086 \quad 3.6153$

C $7.5337-4.4897 \quad 2.8167$

$\begin{array}{llll}\text { N } & 2.9102 & -4.5311 & 2.7608\end{array}$

C $1.8867 \quad-3.8021 \quad 2.0516$

C $0.5141 \quad-4.3663 \quad 2.3999$ $\begin{array}{llll}\text { O } & 0.3648 & -5.1495 & 3.3325\end{array}$

N $-0.4765 \quad-3.8932 \quad 1.6035$

C $-1.8793-4.1408 \quad 1.8903$

$\begin{array}{llll}\text { C } & -2.6286 & -4.6440 & 0.6494\end{array}$

$\begin{array}{lllll}\text { O } & -3.8181 & -4.3485 & 0.4588\end{array}$

$\begin{array}{llll}C & -2.5596 & -2.8877 & 2.4587\end{array}$

$\begin{array}{llll}\mathrm{O} & -3.8452 & -3.1551 & 2.9724\end{array}$

N $-1.9259 \quad-5.4683 \quad-0.1510$

C $-2.4835-6.0480-1.3624$

$\begin{array}{llll}\text { C } & -2.1518 & -5.2039 & -2.5971\end{array}$

$\begin{array}{llll}C & -2.7397 & -5.7499 & -3.9026\end{array}$

$\begin{array}{llll}\text { C } & -4.2611 & -5.9142 & -3.9130\end{array}$

N $-4.9444-4.6475-3.6737$

C $-6.2571-4.5991-3.2829$

$\mathrm{N}-7.0553 \quad-5.6386 \quad-3.4670$

$\begin{array}{llll}\mathrm{N} & -6.7302 & -3.4642 & -2.7669\end{array}$

$\begin{array}{llll}\text { C } & 11.0214 & -4.0097 & -2.6317\end{array}$

C $10.0379-3.9648-1.4645$

C $9.1836-2.7127-1.4636$

$\begin{array}{lllll}\text { O } & 9.2352 & -1.8618 & -2.3359\end{array}$

$\begin{array}{lllll}\text { O } & 8.3693 & -2.6441 & -0.4055\end{array}$

C $2.8015-5.5131-2.8353$

C 2.8638 -4.9118 -4.2362

O $3.8468-4.9960 \quad-4.9638$

C $2.2925-4.5398-1.7507$

C $3.0987-3.2451-1.7044$

O $0.8996-4.2829 \quad-1.9849$

$\begin{array}{llll}\text { N } & 1.7128 & -4.2910 & -4.6448\end{array}$

$\begin{array}{llll}\text { C } & 1.6868 & -3.5913 & -5.8962\end{array}$

$\begin{array}{llll}\text { C } & 2.4475 & -2.2460 & -5.8479\end{array}$

$\begin{array}{llll}\text { O } & 2.6642 & -1.6278 & -4.8018\end{array}$

$\begin{array}{llll}\text { C } & 0.2248 & -3.3938 & -6.3419\end{array}$

O $-0.6156-2.9103-5.3065$

N $2.7960 \quad-1.7502 \quad-7.0586$

C $3.4506-0.4608-7.2257$

C $-5.8151 \quad-2.2153 \quad-7.3919$

C $-4.5381-2.3684-6.5506$

N $-4.2072-1.1261 \quad-5.8396$

C $-3.6069-1.0465-4.6422$

$\mathrm{N}-3.6432 \quad 0.1054 \quad-3.9628$

$\mathrm{N}-3.0217 \quad-2.1246-4.0871$

$\begin{array}{llll}\text { C }-4.2316 & 4.0955 & -5.9525\end{array}$

$\begin{array}{llll}C & -3.2704 & 2.9471 & -6.2216\end{array}$

$\begin{array}{lllll}\text { O } & -3.6862 & 1.7817 & -6.3083\end{array}$

C $-4.4973 \quad 4.1372-4.4269$

C $-3.2678 \quad 4.5737 \quad-3.6256$

N $-2.9109 \quad 3.8091-2.5795$

$\begin{array}{lllll}\mathrm{O} & -2.6668 & 5.6169 & -3.9417\end{array}$

N $-1.95693 .2654-6.2514$

C $-0.8939 \quad 2.2604 \quad-6.1938$

$\begin{array}{llll}\text { C } & 0.1300 & 2.5787 & -5.0936\end{array}$

$\begin{array}{llll}\text { C } & 0.8619 & 1.3449 & -4.5624\end{array}$

$\begin{array}{llll}\text { C } & -0.0489 & 0.4866 & -3.6897\end{array}$

N $0.3467 \quad-0.8101 \quad-3.5059$

$\begin{array}{lllll}\mathrm{O} & -1.0830 & 0.9463 & -3.2095\end{array}$

C $-11.5154-6.4025-0.7292$

C $-11.0415-4.9600-0.9052$

C $-9.7241-4.8058-1.6754$

$\begin{array}{llll}\text { O } & -9.3457 & -5.8051 & -2.3661\end{array}$

O $-9.1313-3.6978-1.5814$ $\begin{array}{llll}\text { C } & -4.1805 & 8.8069 & 4.7003\end{array}$

$\begin{array}{llll}C & -3.8186 & 7.3150 & 4.7268\end{array}$

$\begin{array}{llll}\mathrm{N} & -4.0287 & 6.6098 & 3.4555\end{array}$

$\begin{array}{llll}\mathrm{C} & -3.1062 & 6.4114 & 2.4939\end{array}$

$\begin{array}{llll}\mathrm{N} & -1.9262 & 7.0335 & 2.5337\end{array}$

$\mathrm{N}-3.3861 \quad 5.5956 \quad 1.4710$

C $-0.3002 \quad 11.8672 \quad-3.0700$

$\begin{array}{llll}\text { C } & -0.0140 & 10.7224 & -2.1023\end{array}$

$\begin{array}{llll}\mathrm{N} & -0.7907 & 9.5187 & -2.4270\end{array}$

$\begin{array}{llll}\text { C } & -0.8200 & 8.4232 & -1.6376\end{array}$

$\begin{array}{llll}\mathrm{N} & -0.0537 & 8.3850 & -0.5416\end{array}$

$\mathrm{N}-1.6142 \quad 7.3890 \quad-1.9098$

C $2.3256 \quad 6.2213 \quad-3.3441$

C $3.2628 \quad 5.0382-3.1676$

$\begin{array}{llll}\text { O } & 2.8410 & 3.8942 & -2.9622\end{array}$

C $0.9146 \quad 5.8434 \quad-3.7809$

N $4.5766 \quad 5.3601 \quad-3.2431$

C $5.6387 \quad 4.4991 \quad-2.7610$

C $6.2203 \quad 5.1168-1.4818$

$\begin{array}{lllll}\text { O } & 6.1938 & 6.3383 & -1.3266\end{array}$

N $6.7704 \quad 4.2325 \quad-0.6221$

C $7.3876 \quad 4.6505 \quad 0.6326$

C $\quad 6.7534 \quad 3.9941 \quad 1.8720$

$\begin{array}{llll}\text { C } & 7.1250 & 2.5040 & 1.9987\end{array}$

C $5.2343 \quad 4.2133 \quad 1.9110$

C $\quad \begin{array}{llll}6.9406 & 1.9578 & 3.4164\end{array}$

$\begin{array}{llll}\text { C } & -1.3858 & 2.1632 & 8.9231\end{array}$

$\begin{array}{llll}\text { C } & -0.9795 & 2.4478 & 7.4661\end{array}$

$\begin{array}{llll}\text { C } & -2.0819 & 3.2400 & 6.7381\end{array}$

$\begin{array}{llll}\text { C } & -0.6244 & 1.1549 & 6.7202\end{array}$

C $-1.7126 \quad 3.6905 \quad 5.3195$

C $-1.9385-1.5963 \quad 9.0472$

$\begin{array}{llll}\text { C } & -1.5530 & -2.7657 & 8.1269\end{array}$

$\begin{array}{llll}\text { C } & -0.4389 & -2.4337 & 7.1563\end{array}$

$\begin{array}{llll}\text { C } & -0.6899 & -2.3088 & 5.7848\end{array}$

$\begin{array}{llll}\text { C } & 0.8750 & -2.2466 & 7.6092\end{array}$

$\begin{array}{llll}\text { C } & 0.3411 & -2.0159 & 4.8906\end{array}$

C $1.9089-1.9536 \quad 6.7196$

$\begin{array}{llll}\text { C } & 1.6448 & -1.8403 & 5.3524\end{array}$

$\begin{array}{llll}\text { C } & -7.1074 & -2.1428 & 8.2440\end{array}$

C $-5.9232-2.0571 \quad 7.2669$

$\begin{array}{llll}\text { C } & -5.7181 & -0.6801 & 6.6049\end{array}$

$\begin{array}{llll}\text { C } & -4.4004 & -0.6383 & 5.8144\end{array}$

$\begin{array}{llll}\text { C } & -6.8869 & -0.2821 & 5.6938\end{array}$

C $-1.1187-1.4831-0.6666$

$\begin{array}{llll}\text { C } & -6.7296 & 1.7840 & 2.3399\end{array}$

$\begin{array}{llll}\text { O } & -7.1464 & 0.6460 & 1.7727\end{array}$

$\begin{array}{llll}\text { C } & -5.2372 & 1.9693 & 2.2326\end{array}$

$\begin{array}{llll}\text { O } & -7.4581 & 2.5813 & 2.8943\end{array}$

$\begin{array}{llll}\text { C } & -4.6707 & 2.9913 & 2.9063\end{array}$

$\begin{array}{llll}\text { O } & -4.6435 & 1.0472 & 1.4649\end{array}$

C $5.3844-0.9584-0.0325$

$\begin{array}{llll}\text { C } & 4.2640 & -0.1503 & -0.0836\end{array}$

$\begin{array}{llll}\text { C } & 4.3998 & 1.0674 & -0.8084\end{array}$

N $3.3605 \quad 1.8895 \quad-1.0208$

N $5.6177 \quad 1.4406-1.2800$

C $6.6455 \quad 0.5966-1.1404$

N $6.5736 \quad-0.6110 \quad-0.5582$

C $-1.3892 \quad-0.2170 \quad 0.0473$

O $\quad 0.0240-2.0699-0.5145$ 
$\begin{array}{llll}\mathrm{S} & 1.5910 & 2.0680 & 3.2370\end{array}$

C $\quad 1.6282 \quad 1.1959 \quad 1.7505$

C $7.9891 \quad 1.0373 \quad-1.6383$

$\begin{array}{llll}\text { C } & -0.5419 & 0.0421 & 1.2697\end{array}$

O $-1.9854-1.9313-1.4989$

$\begin{array}{llll}\text { C } & -0.2395 & 2.6557 & 0.9206\end{array}$

$\begin{array}{llll}\text { C } & -1.2653 & 0.2950 & 2.5496\end{array}$

$\begin{array}{llll}\mathrm{N} & 2.6541 & 0.3344 & 1.7414\end{array}$

$\begin{array}{lllll}\mathrm{O} & 0.1394 & 6.0733 & 0.9431\end{array}$

$\begin{array}{lllll}\text { O } & -3.4170 & 0.7306 & -1.0706\end{array}$

$\begin{array}{llll}\text { C } & 3.4292 & 0.3200 & 2.9204\end{array}$

C $4.5837 \quad-0.6096 \quad 3.0790$

$\begin{array}{llll}\text { C } & -2.5073 & 0.7773 & 2.5737\end{array}$

$\begin{array}{lllll}\text { O } & -1.3306 & 5.0577 & -0.4038\end{array}$

C $2.9751 \quad 1.2038 \quad 3.8492$

C $3.4336 \quad 1.4235 \quad 5.2563$

$\begin{array}{llll}\text { C } & -3.2088 & 1.1288 & 1.2938\end{array}$

$\begin{array}{lllll}\text { O } & 0.7308 & 1.0355 & -0.5132\end{array}$

$\begin{array}{llll}\text { C } & -2.8665 & 0.1711 & 0.1370\end{array}$

$\begin{array}{llll}\text { C } & 0.4650 & 1.2890 & 0.8194\end{array}$

$\begin{array}{llll}\text { C } & 2.9799 & -0.5602 & 0.5794\end{array}$

$\begin{array}{llll}\text { C } & 0.5822 & 3.7984 & 0.3044\end{array}$

$\begin{array}{llll}\text { C } & -0.2450 & 5.0647 & 0.2723\end{array}$

H $8.3431 \quad-6.6151 \quad 1.1846$

H $6.1508 \quad-4.4352 \quad 5.3002$

H $5.0321 \quad-3.1974 \quad 4.7143$

H $7.7015 \quad-2.8448 \quad 4.2627$

H $\quad 6.5826 \quad-2.5533 \quad 2.9211$

H $8.3892 \quad-4.9367 \quad 3.3368$

H 7.8588 -4.1777 1.8192

$\begin{array}{llll}\text { H } & 4.7538 & -5.8323 & 3.9369\end{array}$

$\begin{array}{lllll}\mathrm{H} & 1.8931 & -2.7428 & 2.3424\end{array}$

$\begin{array}{llll}\text { H } & 2.0649 & -3.8325 & 0.9748\end{array}$

$\begin{array}{llll}\mathrm{H} & -1.9530 & -2.5173 & 3.2855\end{array}$

H $-2.5720-2.1105 \quad 1.6848$

$\begin{array}{llll}\mathrm{H} & -4.3372 & -3.5332 & 2.2217\end{array}$

H $-1.9185-4.9313 \quad 2.6473$

H $-2.4866-4.1837-2.3945$

H $-1.0658-5.1357-2.6969$

H $-2.3074-6.7343-4.1186$

H $-2.4336-5.0982-4.7300$

$\begin{array}{llll}\mathrm{H} & -4.5588 & -6.6484 & -3.1523\end{array}$

H $-4.5678-6.3221-4.8853$

H $-4.3775-3.8670-3.3655$

H $-8.0731-5.6751-3.0092$

H $-6.6785-6.4582-3.9142$

H $-6.0481-2.8065-2.3940$

H $-7.6983-3.5047-2.3367$

H $-3.5620-6.1105-1.2043$

H $10.4914-3.9613-3.5856$

H $11.6094-4.9312-2.6055$

H $10.5534-4.0169-0.4978$

H $9.3564 \quad-4.8248-1.4812$

$\begin{array}{llll}\text { H } & 2.4009 & -5.0608 & -0.7869\end{array}$

H $2.6397 \quad-2.5599-0.9912$

$\begin{array}{llll}\text { H } & 4.1246 & -3.4419 & -1.3824\end{array}$

H $3.1181-2.7546-2.6809$

H $\quad 0.5861 \quad-3.4952 \quad-1.4727$

H $\quad 2.1455 \quad-6.3909-2.8532$

H $-0.1830 \quad-4.3675 \quad-6.6238$
H $0.1942-2.7386 \quad-7.2244$

H $-0.2581-2.0897 \quad-4.9066$

H $2.1922-4.2122 \quad-6.6454$

H $3.6273-0.0502-6.2323$

H $2.8213 \quad 0.2274 \quad-7.8001$

H $-6.6725-1.9895-6.7531$

H $-5.7090 \quad-1.4197 \quad-8.1369$

H $-4.6790-3.1502-5.7985$

H $-3.6975-2.6658-7.1918$

H $-4.4121 \quad-0.2389-6.2871$

$\begin{array}{llll}\mathrm{H} & -2.9146 & 0.2704 & -3.2636\end{array}$

H $-3.9394 \quad 0.9139 \quad-4.4976$

H $-2.5437-1.9800 \quad-3.1846$

H $-2.5026-2.7245 \quad-4.7230$

H $-4.8748 \quad 3.1680 \quad-4.0869$

H $-5.2788 \quad 4.8769 \quad-4.2217$

H $-3.3253 \quad 2.9100 \quad-2.3641$

H $-2.1430 \quad 4.1049 \quad-1.9785$

$\begin{array}{llll}\mathrm{H} & -3.8250 & 5.0587 & -6.2703\end{array}$

H $\quad 0.8652 \quad 3.3014 \quad-5.4584$

H $-0.3955 \quad 3.0343-4.2515$

H $\quad 1.7010 \quad 1.6710 \quad-3.9354$

H $1.2951 \quad 0.7338 \quad-5.3612$

H $-0.0543-1.3075 \quad-2.7169$

H $1.3063-1.0668$ - 3.7458

H $-0.4054 \quad 2.1747 \quad-7.1718$

$\mathrm{H}-1.7134 \quad 4.2270 \quad-6.0519$

H - $12.4778-6.4412 \quad-0.2071$

H -11.6211 -6.8911 - -1.7002

H -11.7953 $-4.3740-1.4485$

H $-10.9169-4.4558 \quad 0.0594$

H $-5.2329 \quad 8.9486 \quad 4.4407$

H $-3.5761 \quad 9.3552 \quad 3.9708$

H $-4.4236 \quad 6.7949 \quad 5.4739$

H $-2.7768 \quad 7.1630 \quad 5.0263$

$\begin{array}{llll}\mathrm{H} & -4.9446 & 6.2213 & 3.2817\end{array}$

H $-1.7517 \quad 7.7403 \quad 3.2280$

H $-1.1459 \quad 6.7160 \quad 1.9287$

H $-4.0847 \quad 4.8700 \quad 1.5990$

H $-2.6845 \quad 5.4459 \quad 0.7302$

H $-1.3536 \quad 12.1609$ - 3.0343

H $\quad 0.3021 \quad 12.7351 \quad-2.7949$

H $-0.2583 \quad 11.0453-1.0815$

H $1.053910 .4726-2.1291$

H $-1.4828 \quad 9.5794 \quad-3.1591$

H $-1.5458 \quad 6.5537-1.3062$

H $-1.9943 \quad 7.1876 \quad-2.8329$

H $\quad 0.0221 \quad 7.5169 \quad 0.0215$

H $\quad 0.5379 \quad 9.1638 \quad-0.3090$

H $\quad 0.4536 \quad 5.1472 \quad-3.0784$

H $\quad 0.2841 \quad 6.7327 \quad-3.8444$

H $\quad 0.9222 \quad 5.3685-4.7636$

H $2.2879 \quad 6.7309 \quad-2.3701$

H $\quad 5.2691 \quad 3.4868 \quad-2.6064$

H $6.4412 \quad 4.4450 \quad-3.5067$

H $7.2005 \quad 4.5129 \quad 2.7324$

H $4.8007 \quad 3.8323 \quad 2.8407$

H $4.9872 \quad 5.2770 \quad 1.8368$

H $4.7421 \quad 3.6982 \quad 1.0791$

H $\quad 6.5252 \quad 1.9145 \quad 1.2943$
H $\quad 8.1729 \quad 2.3673 \quad 1.7031$

H $5.8974 \quad 2.0283 \quad 3.7376$

H $7.2428 \quad 0.9085 \quad 3.4827$

H $7.5472 \quad 2.5226 \quad 4.1323$

H $7.2734 \quad 5.7354 \quad 0.6752$

H $-0.0797 \quad 3.0826 \quad 7.4900$

H $\quad 0.1266 \quad 0.5706 \quad 7.2590$

$\begin{array}{llll}\mathrm{H} & -1.5027 & 0.5121 & 6.5977\end{array}$

$\mathrm{H}-0.2270 \quad 1.3528 \quad 5.7193$

$\begin{array}{llll}\mathrm{H} & -2.3359 & 4.1228 & 7.3397\end{array}$

H $-2.9916 \quad 2.6249 \quad 6.6981$

H $-2.49394 .3315 \quad 4.8946$

H $-0.7759 \quad 4.2617 \quad 5.3162$

H $-1.5840 \quad 2.8407 \quad 4.6423$

H $-1.5652 \quad 3.0928 \quad 9.4732$

$\mathrm{H}-1.2478-3.6203 \quad 8.7430$

$\begin{array}{llll}\mathrm{H} & -2.4349 & -3.0903 & 7.5647\end{array}$

H $-1.7004 \quad-2.4579 \quad 5.4165$

$\begin{array}{llll}\text { H } & 1.0918 & -2.3485 & 8.6693\end{array}$

H $\quad 0.1250 \quad-1.9378 \quad 3.8302$

H $2.9227 \quad-1.8326 \quad 7.0914$

H $2.4474 \quad-1.6327 \quad 4.6529$

H $-2.3154 \quad-0.7509 \quad 8.4658$

$\begin{array}{llll}\mathrm{H} & -5.0031 & -2.3182 & 7.8074\end{array}$

H $-6.0365 \quad-2.81396 .4789$

H $-5.6493 \quad 0.0647 \quad 7.4132$

H $-4.4012-1.3646 \quad 4.9924$

$\begin{array}{llll}\mathrm{H} & -3.5405 & -0.8544 & 6.4575\end{array}$

H $-4.2436 \quad 0.3580 \quad 5.3825$

H $-7.8432-0.2876 \quad 6.2241$

H $-6.7417 \quad 0.7259 \quad 5.2894$

H $-6.9660 \quad-0.9734 \quad 4.8462$

H $-8.0644 \quad-1.9866 \quad 7.7372$

H $-5.3218 \quad 3.6060 \quad 3.5140$

H $-3.6024 \quad 3.1355 \quad 2.9666$

H $5.3365 \quad-1.9293 \quad 0.4523$

H $2.4004 \quad 1.5494-0.8925$

H $-0.3679 \quad 0.5381 \quad-0.7108$

H $7.8870 \quad 1.8478 \quad-2.3610$

$\begin{array}{llll}\text { H } & 8.5921 & 1.3992 & -0.7961\end{array}$

H $8.5172 \quad 0.1874-2.0780$

H $0.1448 \quad-0.7990 \quad 1.3916$

H $-0.5425 \quad 2.8963 \quad 1.9460$

H $-1.1494 \quad 2.5534 \quad 0.3296$

$\begin{array}{llll}\mathrm{H} & -0.7713 & 0.0460 & 3.4841\end{array}$

H $-4.1699 \quad 0.1540 \quad-1.2860$

H $4.8717 \quad-0.6581 \quad 4.1290$

H $4.3492 \quad-1.6193 \quad 2.7389$

H $\quad 5.4481 \quad-0.2706 \quad 2.5054$

H $\quad-3.0356 \quad 0.9010 \quad 3.5114$

H $2.8144 \quad 0.8435 \quad 5.9498$

$\begin{array}{llll}\mathrm{H} & 4.4728 & 1.1152 & 5.3802\end{array}$

H $-2.9898 \quad 2.1586 \quad 0.9801$

$\begin{array}{llll}\mathrm{H} & -3.4484 & -0.7307 & 0.3521\end{array}$

H $3.0891 \quad-1.5680 \quad 0.9754$

H $2.1113-0.5302 \quad-0.0789$

H $1.5038 \quad 3.9910 \quad 0.8575$

$\mathrm{H} \quad 0.8402 \quad 3.5150 \quad-0.7210$

H $-10.7906-6.9848 \quad-0.1521$

H $-4.0090 \quad 9.2445 \quad 5.6874$ 
H $-2.3052 \quad 1.5688 \quad 8.9630$ H $-0.6061 \quad 1.6025 \quad 9.4478$ $\begin{array}{llll}\mathrm{H} & -7.1460 & -3.1247 & 8.7236\end{array}$ H $-7.0204-1.3868 \quad 9.0325$ H $-2.7185-1.8993 \quad 9.7523$ H $-1.0785-1.2438 \quad 9.6258$ H $11.7071-3.1594-2.5959$ H $7.8064 \quad-8.2705 \quad 1.5848$ H $\quad 8.5815 \quad-7.2473 \quad 2.8175$ H $-6.0142 \quad-3.1496-7.9219$ H $3.4574 \quad 2.6420-1.7006$ H $2.7731 \quad 6.9363 \quad-4.0438$

H $7.7816-1.8278-0.4943$ $\begin{array}{llll}\text { O } & -4.6434 & -1.9670 & -1.2974\end{array}$ H $-3.6553-2.0159-1.3567$ H $-4.8598 \quad-2.5566-0.5599$ $\begin{array}{llll}\text { H } & 3.3649 & 2.4777 & 5.5381\end{array}$ H $8.4613 \quad 4.4261 \quad 0.6073$ H $-0.0462 \quad 11.5903-4.0973$ $\begin{array}{llll}\mathrm{H} & -8.1079 & 0.5958 & 1.9090\end{array}$ H $2.6986-2.3479-7.8663$ H $4.4098 \quad-0.5769-7.7379$ $\begin{array}{llll}\text { H } & 3.8097 & -5.8462 & -2.5849\end{array}$ H 1.0139 -4.0818 -3.9350 H $2.5791 \quad-5.2012 \quad 3.4465$ H $-0.2456 \quad-3.2209 \quad 0.8571$ $\begin{array}{llll}\mathrm{H} & -0.9225 & -5.4856 & -0.0291\end{array}$ H $4.8126 \quad 6.3436-3.1689$ $\begin{array}{lllll}\text { H } & 6.5711 & 3.2497 & -0.7940\end{array}$ $\begin{array}{llll}\mathrm{H} & -2.0957 & -7.0678 & -1.4686\end{array}$ $\begin{array}{llll}\mathrm{H} & -5.1616 & 3.8980 & -6.4868\end{array}$ $\begin{array}{llll}\text { H } & -1.3968 & 1.3122 & -5.9932\end{array}$

\section{Int5}

C $7.5859 \quad-7.6764 \quad 1.2884$

C $6.2903 \quad-7.0974 \quad 1.8353$

$\begin{array}{llll}\text { O } & 5.2421 & -7.7350 & 1.8776\end{array}$

$\begin{array}{llll}\mathrm{N} & 6.3577 & -5.8168 & 2.3205\end{array}$

$\begin{array}{llll}\text { C } & 5.2525 & -5.2944 & 3.1181\end{array}$

$\begin{array}{llll}\text { C } & 4.2101 & -4.5535 & 2.2768\end{array}$

$\begin{array}{llll}\text { O } & 4.5300 & -3.7002 & 1.4373\end{array}$

C $5.9611 \quad-4.2988 \quad 4.0638$

$\begin{array}{llll}\text { C } & 7.1224 & -3.7627 & 3.2112\end{array}$

$\begin{array}{llll}\text { C } & 7.5621 & -4.9753 & 2.3758\end{array}$

$\begin{array}{llll}\text { N } & 2.9178 & -4.7935 & 2.5692\end{array}$

C $1.8713 \quad-4.0323 \quad 1.9339$

$\begin{array}{llll}\text { C } & 0.5075 & -4.5437 & 2.3831\end{array}$

$\begin{array}{lllll}\text { O } & 0.3850 & -5.3518 & 3.2945\end{array}$

N $-0.5256 \quad-3.9956 \quad 1.6871$

C $-1.9160-4.1461 \quad 2.0800$

$\begin{array}{llll}\text { C } & -2.8041 & -4.6071 & 0.9194\end{array}$

$\begin{array}{llll}\text { O } & -4.0319 & -4.3987 & 0.9600\end{array}$

$\begin{array}{llll}\text { C } & -2.3874 & -2.8109 & 2.7018\end{array}$

$\begin{array}{llll}\text { O } & -3.7652 & -2.7048 & 2.9643\end{array}$

$\begin{array}{llll}\mathrm{N} & -2.2071 & -5.2988 & -0.0619\end{array}$

$\begin{array}{llll}\text { C } & -2.9571 & -5.8882 & -1.1589\end{array}$

$\begin{array}{llll}\text { C } & -2.6559 & -5.2327 & -2.5083\end{array}$

$\begin{array}{llll}\text { C } & -3.4052 & -5.8729 & -3.6843\end{array}$

$\begin{array}{llll}\text { C } & -4.9248 & -5.9810 & -3.5317\end{array}$
N $-5.5429-4.6754 \quad-3.3445$

C $-6.8332-4.5161 \quad-2.9256$

$\mathrm{N}-7.7063 \quad-5.5045 \quad-3.0167$

$\begin{array}{llll}\mathrm{N} & -7.2098 & -3.3142 & -2.4665\end{array}$

C $10.4863-4.6771 \quad-3.5999$

C $9.7554-4.5649 \quad-2.2641$

C $8.9148-3.3069-2.1650$

$\begin{array}{lllll}\text { O } & 8.7455 & -2.5407 & -3.0996\end{array}$

$\begin{array}{lllll}\text { O } & 8.3844 & -3.1291 & -0.9522\end{array}$

C $2.2051 \quad-5.6872 \quad-3.0832$

C $2.1107-4.9928-4.4434$

$\begin{array}{lllll}\text { O } & 2.9782 & -5.0870 & -5.3048\end{array}$

C $1.8285-4.7913-1.8825$

C $2.5946-3.4685-1.8483$

$\begin{array}{lllll}\mathrm{O} & 0.4048 & -4.5827 & -1.9225\end{array}$

N $0.9697 \quad-4.2663 \quad-4.6571$

C $0.7905-3.5424 \quad-5.8846$

C $1.6642-2.2699-5.9885$

O $1.9349-1.5594 \quad-5.0160$

C $-0.7058-3.1961 \quad-6.0581$

O $-1.3320-2.7358-4.8713$

$\begin{array}{llll}\mathrm{N} & 2.0229 & -1.9378 & -7.2530\end{array}$

C $2.7722-0.7312-7.5925$

C $-6.5607-1.9287-6.9455$

C $-5.2022-2.0637-6.2298$

$\begin{array}{llll}\mathrm{N} & -4.8758 & -0.8914 & -5.4008\end{array}$

$\begin{array}{llll}\text { C } & -4.3967 & -0.9279 & -4.1460\end{array}$

$\begin{array}{llll}\mathrm{N} & -4.5107 & 0.1382 & -3.3588\end{array}$

$\begin{array}{llll}\mathrm{N} & -3.8359 & -2.0609 & -3.6630\end{array}$

$\begin{array}{llll}\text { C } & -4.4915 & 4.2912 & -5.7463\end{array}$

C $-\begin{array}{llll}-3.6223 & 3.0542 & -5.9105\end{array}$

$\begin{array}{lllll}\text { O } & -4.0537 & 1.9398 & -5.5846\end{array}$

$\begin{array}{llll}\text { C } & -4.6933 & 4.5626 & -4.2388\end{array}$

$\begin{array}{llll}\mathrm{C} & -3.4346 & 5.0772 & -3.5378\end{array}$

$\begin{array}{llll}\mathrm{N} & -2.7057 & 4.1782 & -2.8453\end{array}$

$\begin{array}{lllll}\mathrm{O} & -3.1423 & 6.2794 & -3.6181\end{array}$

$\begin{array}{llll}\mathrm{N} & -2.3608 & 3.2581 & -6.3555\end{array}$

C $-1.3007 \quad 2.2566 \quad-6.2395$

$\begin{array}{llll}C & -0.2576 & 2.6580 & -5.1843\end{array}$

C $0.4392 \quad 1.4752 \quad-4.5195$

$\begin{array}{llll}\text { C } & -0.4657 & 0.7113 & -3.5626\end{array}$

$\begin{array}{llll}\mathrm{N} & -0.0922 & -0.5898 & -3.3444\end{array}$

$\begin{array}{lllll}\mathrm{O} & -1.4509 & 1.2243 & -3.0271\end{array}$

$\begin{array}{llll}\mathrm{C}-11.9070 & -5.6939 & 0.2419\end{array}$

C -11.6059 $-4.3783-0.4805$

C - $10.2783-4.3667-1.2469$

$\begin{array}{lllll}\text { O } & -9.9108 & -5.4752 & -1.7589\end{array}$

$\begin{array}{llll}\text { O } & -9.6706 & -3.2690 & -1.3421\end{array}$

$\begin{array}{llll}\text { C } & -3.2534 & 9.1067 & 4.7874\end{array}$

C $-2.8450 \quad 7.6266 \quad 4.7896$

$\begin{array}{llll}\mathrm{N} & -3.2211 & 6.8835 & 3.5787\end{array}$

C $-2.4290 \quad 6.6029 \quad 2.5253$

N -1.2339 $7.1790 \quad 2.3814$

$\begin{array}{llll}\mathrm{N} & -2.8598 & 5.7437 & 1.5936\end{array}$

$\begin{array}{llll}\text { C } & 0.1217 & 11.8441 & -3.3314\end{array}$

C $0.5369 \quad 10.6534 \quad-2.4781$

$\begin{array}{lllll}\mathrm{N} & -0.4594 & 9.5820 & -2.5506\end{array}$

C $-0.4677 \quad 8.5085-1.7333$

N $\quad 0.5294 \quad 8.3396 \quad-0.8559$

$\mathrm{N}-1.4685 \quad 7.6332 \quad-1.7651$ $\begin{array}{llll}\text { C } & 2.3780 & 6.0484 & -3.7367\end{array}$

C $3.2065 \quad 4.7883 \quad-3.5081$

$\begin{array}{lllll}\text { O } & 2.7213 & 3.7380 & -3.0738\end{array}$

$\begin{array}{llll}\text { C } & 0.8720 & 5.8190 & -3.7897\end{array}$

N $4.5183 \quad 4.9413-3.8245$

C $5.5475 \quad 4.0365 \quad-3.3512$

C $6.2654 \quad 4.6740-2.1503$

$\begin{array}{lllll}\text { O } & 6.3111 & 5.9023 & -2.0576\end{array}$

N $6.8549 \quad 3.7961 \quad-1.3160$

C $7.6602 \quad 4.2203 \quad-0.1783$

$\begin{array}{llll}\text { C } & 7.1241 & 3.7631 & 1.1915\end{array}$

C $7.4009 \quad 2.2718 \quad 1.4660$

C $5.6434 \quad 4.1234 \quad 1.3697$

$\begin{array}{llll}\text { C } & 7.3171 & 1.9107 & 2.9509\end{array}$

$\begin{array}{llll}C & -0.5035 & 2.3538 & 8.8639\end{array}$

$\begin{array}{llll}\text { C } & -0.1684 & 2.6009 & 7.3820\end{array}$

C $-1.1627 \quad 3.5936 \quad 6.7505$

$\begin{array}{llll}\text { C } & -0.1147 & 1.2814 & 6.6007\end{array}$

C $-0.8093 \quad 4.0221 \quad 5.3220$

C $-1.2636-1.3641 \quad 9.0943$

$\begin{array}{llll}\text { C } & -0.6744 & -2.6235 & 8.4348\end{array}$

C $\quad 0.2370 \quad-2.3176 \quad 7.2663$

C $-0.2482-2.3320 \quad 5.9531$

$\begin{array}{llll}\text { C } & 1.5838 & -1.9860 & 7.4699\end{array}$

$\begin{array}{llll}\text { C } & 0.5811 & -2.0176 & 4.8772\end{array}$

C $2.4186 \quad-1.6737 \quad 6.3962$

C $\quad 1.9178-1.6872 \quad 5.0930$

C $-6.5053-1.6085 \quad 8.7406$

C $-5.5289-1.6686 \quad 7.5563$

$\begin{array}{llll}\text { C } & -5.0241 & -0.3019 & 7.0554\end{array}$

C $-3.9684-0.4714 \quad 5.9520$

$\begin{array}{llll}\text { C } & -6.1652 & 0.5936 & 6.5568\end{array}$

$\begin{array}{llll}\text { C } & -1.7080 & -1.7271 & -0.6652\end{array}$

$\begin{array}{llll}\text { C } & -6.0734 & 2.3405 & 3.4880\end{array}$

O

$\begin{array}{llll}\text { C } & -4.6573 & 2.2355 & 2.9922\end{array}$

O $-6.4834 \quad 3.2915 \quad 4.1231$

C $-3.7798 \quad 3.1916 \quad 3.3561$

$\begin{array}{llll}\text { O } & -4.4353 & 1.1609 & 2.2189\end{array}$

C $5.5969-1.2190-0.1553$

$\begin{array}{llll}\text { C } & 4.5436 & -0.3262 & -0.0807\end{array}$

$\begin{array}{llll}\text { C } & 4.6571 & 0.8395 & -0.8901\end{array}$

N $3.6745 \quad 1.7548 \quad-0.9884$

N $5.7992 \quad 1.0735-1.5820$

$\begin{array}{llll}\text { C } & 6.7825 & 0.1684 & -1.5336\end{array}$

N $6.7168-0.9935-0.8676$

C $-1.8256-0.2896-0.0982$

$\begin{array}{llll}\text { O } & -0.5534 & -2.2492 & -0.7225\end{array}$

$\begin{array}{llll}\text { S } & 2.4171 & 2.0876 & 3.4385\end{array}$

C $2.2580 \quad 1.2382 \quad 1.9436$

$\begin{array}{llll}\text { C } & 8.0601 & 0.4845 & -2.2489\end{array}$

$\begin{array}{llll}\text { C } & -0.5734 & 0.0933 & 0.7445\end{array}$

$\begin{array}{llll}\text { O } & -2.7583 & -2.2758 & -1.1038\end{array}$

$\begin{array}{llll}C & -0.1843 & 2.6569 & 0.4531\end{array}$

$\begin{array}{llll}\text { C } & -0.8739 & 0.3333 & 2.1981\end{array}$

$\begin{array}{llll}\text { N } & 3.2193 & 0.2968 & 1.9451\end{array}$

$\begin{array}{lllll}\mathrm{O} & 0.6177 & 6.0061 & 0.6210\end{array}$

$\begin{array}{llll}\text { O } & -4.1599 & 0.2942 & -0.4581\end{array}$

$\begin{array}{llll}C & 4.0828 & 0.2039 & 3.0650\end{array}$

C $5.1444 \quad-0.8436 \quad 3.1340$ 
$\begin{array}{llll}C & -2.0646 & 0.7626 & 2.6136\end{array}$

$\begin{array}{lllll}\text { O } & -1.1055 & 5.2174 & -0.5659\end{array}$

C $3.7701 \quad 1.1277 \quad 4.0117$

C $4.3644 \quad 1.3336 \quad 5.3679$

C $\quad-3.1412 \quad 1.0616 \quad 1.6054$

$\begin{array}{lllll}\mathrm{O} & 0.8922 & 0.9981 & -0.9111\end{array}$

$\begin{array}{llll}\text { C } & -3.2113 & -0.0497 & 0.5518\end{array}$

$\begin{array}{llll}\text { C } & 0.1207 & 1.2530 & 0.0070\end{array}$

$\begin{array}{llll}\text { C } & 3.3400 & -0.6143 & 0.7743\end{array}$

$\begin{array}{llll}\text { C } & 0.7172 & 3.7280 & -0.1590\end{array}$

$\begin{array}{lllll}C & 0.0524 & 5.0774 & -0.0301\end{array}$

H $8.0182 \quad-7.0374 \quad 0.5118$

H $6.3406 \quad-4.8495 \quad 4.9301$

H $5.2912 \quad-3.5171 \quad 4.4316$

H $7.9404 \quad-3.3592 \quad 3.8129$

$\begin{array}{llll}\text { H } & 6.7653 & -2.9781 & 2.5437\end{array}$

H $8.3848 \quad-5.51512 .8612$

H $7.8873-4.6800 \quad 1.3731$

H $4.7726-6.1220 \quad 3.6456$

H $1.9457 \quad-2.9713 \quad 2.2071$

H $1.9609 \quad-4.0822 \quad 0.8451$

H $-1.8534-2.6782 \quad 3.6467$

H $-2.0441-2.0095 \quad 2.0358$

$\begin{array}{llll}\text { H } & -4.1988 & -3.3018 & 2.3193\end{array}$

$\begin{array}{llll}\mathrm{H} & -1.9528 & -4.9407 & 2.8325\end{array}$

H $-2.8805-4.1702-2.4129$

H $-1.5837-5.2912-2.7049$

$\begin{array}{llll}\mathrm{H} & -3.0281 & -6.8884 & -3.8538\end{array}$

H $-3.1747-5.3071-4.5965$

$\begin{array}{llll}\mathrm{H} & -5.1740 & -6.6316 & -2.6833\end{array}$

H $-5.3306-6.4643-4.4312$

$\begin{array}{llll}\mathrm{H} & -4.9461 & -3.8621 & -3.2564\end{array}$

H $-8.6993-5.4595-2.4811$

H $-7.4162-6.3520-3.4787$

H $-6.4996-2.7771-1.9709$

H $-8.1826-3.2671-2.0538$

H $-4.0106-5.7744-0.8986$

H $9.7766-4.6655-4.4300$

H $11.0663-5.6032-3.6480$

H $10.4512-4.5711-1.4170$

H $9.0862 \quad-5.4199-2.1018$

H $2.0689-5.3621 \quad-0.9735$

H $2.2211 \quad-2.8337 \quad-1.0387$

H $3.6580 \quad-3.6468-1.6668$

$\begin{array}{llll}\text { H } & 2.4839 & -2.9172 & -2.7859\end{array}$

H $0.1409-3.7286-1.5152$

H $1.5451 \quad-6.5618-3.0816$

H $-1.2366-4.1118-6.3309$

H $-0.8175-2.4764-6.8815$

H $-0.9061-1.9215-4.5242$

H $1.0783-4.1977-6.7158$

H $2.9694-0.1941-6.6650$

H $2.1966-0.0945-8.2718$

H $-7.3728-1.8485-6.2190$

H $-6.5812-1.0514-7.6002$

H $-5.2226-2.9324-5.5676$

H $-4.4051-2.2237-6.9666$

H $-5.0363 \quad 0.0358 \quad-5.7794$

$\begin{array}{llll}\mathrm{H} & -4.0707 & 0.1747 & -2.4364\end{array}$

H $-4.8431 \quad 0.9979 \quad-3.7714$
H $-3.4341 \quad-1.9982-2.7179$

H $-3.1981-2.5323-4.3069$

H $-5.0662 \quad 3.6528 \quad-3.7608$

H $-5.4467 \quad 5.3436-4.1160$

H $-2.8599 \quad 3.1798 \quad-2.9011$

H $-1.9438 \quad 4.4910-2.2451$

H $-4.0579 \quad 5.1736 \quad-6.2263$

H $\quad 0.4981 \quad 3.3083 \quad-5.6360$

H $-0.7612 \quad 3.2320-4.4040$

H $\quad 1.2671 \quad 1.8534 \quad-3.9051$

H $\quad 0.8845 \quad 0.7839 \quad-5.2423$

H $-0.3227-1.0000 \quad-2.4441$

H $0.8160 \quad-0.8844 \quad-3.7072$

$\begin{array}{llll}\mathrm{H} & -0.8390 & 2.0932 & -7.2191\end{array}$

H $-2.0832 \quad 4.2135-6.5310$

H -12.8943 $\quad-5.6689 \quad 0.7147$

H $-11.8731-6.5264-0.4642$

H $-12.3948-4.1755-1.2183$

H $-11.6024 \quad-3.5285 \quad 0.2086$

H $-4.3368 \quad 9.2125 \quad 4.6859$

H $-2.7809 \quad 9.6528 \quad 3.9649$

H $-3.3159 \quad 7.1095 \quad 5.6300$

$\mathrm{H}-1.7667 \quad 7.5088 \quad 4.9327$

$\begin{array}{llll}\mathrm{H} & -4.1675 & 6.5333 & 3.5266\end{array}$

H $-0.9537 \quad 7.9235 \quad 2.9975$

$\begin{array}{llll}\mathrm{H} & -0.5391 & 6.7860 & 1.7208\end{array}$

H $-3.5352 \quad 5.0342 \quad 1.8584$

H $-2.2769 \quad 5.5598 \quad 0.7631$

H $-0.8274 \quad 12.2646 \quad-2.9854$

H $\quad 0.8802 \quad 12.6272 \quad-3.2695$

H $\quad 0.6457 \quad 10.9770-1.4346$

$\begin{array}{lllll}\mathrm{H} & 1.5087 & 10.2698 & -2.8137\end{array}$

H $-1.2856 \quad 9.7379 \quad-3.1090$

$\begin{array}{llll}\mathrm{H} & -1.3564 & 6.7617 & -1.2148\end{array}$

$\begin{array}{llll}\mathrm{H} & -2.0663 & 7.4992 & -2.5827\end{array}$

H $\quad 0.5871 \quad 7.4739-0.2900$

H $1.2919 \quad 8.9936-0.8179$

H $\quad 0.5205 \quad 5.2914 \quad-2.9018$

H $\quad 0.3412 \quad 6.7720 \quad-3.8565$

H $\quad 0.5933 \quad 5.2219 \quad-4.6584$

H $2.6211 \quad 6.7258 \quad-2.9055$

H $5.1166 \quad 3.0618-3.1299$

H $6.2906 \quad 3.8859 \quad-4.1430$

H $7.7086 \quad 4.3344 \quad 1.9279$

H $\quad \begin{array}{llll}5.2989 & 3.9008 & 2.3839\end{array}$

H $5.4728 \quad 5.1881 \quad 1.1813$

H $\quad 5.0148 \quad 3.5564 \quad 0.6765$

H $6.6964 \quad 1.6531 \quad 0.8980$

H $\quad 8.4026 \quad 2.0202 \quad 1.0946$

H $\quad 6.3053 \quad 2.0540 \quad 3.3383$

H $7.5946 \quad 0.8665 \quad 3.1224$

H $7.9963 \quad 2.5340 \quad 3.5427$

H $7.6884 \quad 5.3108 \quad-0.2234$

$\begin{array}{llll}\mathrm{H} & 0.8305 & 3.0624 & 7.3378\end{array}$

$\begin{array}{lllll}\mathrm{H} & 0.5579 & 0.5627 & 7.0744\end{array}$

$\begin{array}{llll}\mathrm{H} & -1.1069 & 0.8182 & 6.5533\end{array}$

H $\quad 0.2367 \quad 1.4232 \quad 5.5743$

H $-1.2241 \quad 4.4860 \quad 7.3875$

$\begin{array}{llll}\mathrm{H} & -2.1651 & 3.1425 & 6.7577\end{array}$

H $-1.5306 \quad 4.7536 \quad 4.9394$
H $\quad 0.1862 \quad 4.4789 \quad 5.2792$

H $-0.8070 \quad 3.1727 \quad 4.6325$

$\begin{array}{llll}\mathrm{H} & -0.5075 & 3.2881 & 9.4349\end{array}$

H $-0.1195 \quad-3.1941 \quad 9.1884$

H $-1.4923-3.2685 \quad 8.0954$

H $-1.2843-2.6040 \quad 5.7741$

H $1.9807 \quad-1.9797 \quad 8.4818$

H $\quad 0.1817 \quad-2.0364 \quad 3.8695$

H $3.4614 \quad-1.4286 \quad 6.5758$

H $2.5617-1.4401 \quad 4.2576$

H $-1.8615 \quad-0.7873 \quad 8.3818$

H $-4.6578-2.2726 \quad 7.8428$

H $-6.0005-2.1981 \quad 6.7170$

H $-4.5453 \quad 0.2055 \quad 7.9076$

H $-4.3701 \quad-1.0010 \quad 5.0807$

$\begin{array}{llll}\mathrm{H} & -3.0984 & -1.0360 & 6.3070\end{array}$

$\mathrm{H} \quad-3.6147 \quad 0.5097 \quad 5.6121$

$\begin{array}{llll}\mathrm{H} & -6.9193 & 0.7703 & 7.3288\end{array}$

$\begin{array}{llll}\mathrm{H} & -5.7881 & 1.5713 & 6.2389\end{array}$

H $-6.6633 \quad 0.1309 \quad 5.6965$

H $-7.4454 \quad-1.1185 \quad 8.4704$

H $-4.1273 \quad 3.9556 \quad 4.0383$

H $-2.7319 \quad 3.1593 \quad 3.0985$

H $5.5440 \quad-2.1657 \quad 0.3755$

H $2.7409 \quad 1.5243 \quad-0.6681$

H $-1.8403 \quad 0.3304-1.0054$

H $7.9285 \quad 1.3366 \quad-2.9165$

H $8.83640 .7319 \quad-1.5147$

H $8.4002 \quad-0.3962-2.7998$

H $\quad 0.1202 \quad-0.7440 \quad 0.6595$

H $-0.1382 \quad 2.6905 \quad 1.5436$

$\mathrm{H}-1.2261 \quad 2.8272 \quad 0.1655$

H $-0.0786 \quad 0.1346 \quad 2.9069$

$\begin{array}{llll}\mathrm{H} & -4.8620 & -0.3902 & -0.3956\end{array}$

H $5.5434 \quad-0.9114 \quad 4.1469$

H $4.7604 \quad-1.8253 \quad 2.8500$

H $5.9740 \quad-0.6156 \quad 2.4607$

H $-2.2846 \quad 0.8759 \quad 3.6692$

$\begin{array}{llll}\mathrm{H} & 3.6343 & 1.0995 & 6.1501\end{array}$

$\begin{array}{llll}\mathrm{H} & 5.2368 & 0.6935 & 5.5162\end{array}$

H $-2.9567 \quad 2.0189 \quad 1.0954$

H $-3.5591 \quad-0.9448 \quad 1.0760$

H $3.4015 \quad-1.6446 \quad 1.1284$

H $2.4143 \quad-0.4909 \quad 0.2097$

$\begin{array}{llll}\mathrm{H} & 1.6822 & 3.7415 & 0.3501\end{array}$

H $\quad 0.8974 \quad 3.5087 \quad-1.2172$

$\mathrm{H}-11.1640 \quad-5.8934 \quad 1.0214$

H $-2.9511 \quad 9.5715 \quad 5.7295$

H $-1.4920 \quad 1.8919 \quad 8.9666$

H $\quad 0.2259 \quad 1.6784 \quad 9.3214$

H $-6.7499-2.6144 \quad 9.0945$

H $-6.0711-1.0553 \quad 9.5814$

H $-1.9088-1.6350 \quad 9.9362$

H $-0.4730-0.7091 \quad 9.4709$

H $11.1681 \quad-3.8347 \quad-3.7424$

H $7.3702 \quad-8.6607 \quad 0.8752$

H $\quad 8.3298 \quad-7.7746 \quad 2.0863$

H $-6.7287-2.8152-7.5621$

H $3.6945 \quad 2.4272 \quad-1.7483$

H $2.7308 \quad 6.5468 \quad-4.6467$ 
H $7.8133-2.2938-0.9631$

O $\quad-5.3622 \quad-2.1243 \quad-0.4961$

$\begin{array}{llll}\mathrm{H} & -4.4179 & -2.3679 & -0.6336\end{array}$

$\begin{array}{llll}\mathrm{H} & -5.6305 & -2.6219 & 0.2876\end{array}$

$\begin{array}{llll}\mathrm{H} & 4.6872 & 2.3700 & 5.5086\end{array}$

H $8.6863 \quad 3.8527 \quad-0.3076$

H $\quad 0.0175 \quad 11.5605 \quad-4.3831$

$\begin{array}{llll}\mathrm{H} & -7.7012 & 1.4284 & 3.5372\end{array}$

$\begin{array}{llll}\text { H } & 1.8955 & -2.6330 & -7.9738\end{array}$

H $3.7250 \quad-0.9885-8.0630$

H $3.2335-6.0383-2.9803$

H $0.3302-4.1204-3.8816$

H $2.6186-5.5020 \quad 3.2295$

H $-0.3312-3.31140 .9569$

H $-1.1965-5.3030-0.1127$

H $4.8492 \quad 5.8975 \quad-3.8904$

$\begin{array}{lllll}\mathrm{H} & 6.6023 & 2.8117 & -1.4317\end{array}$

H $-2.7367-6.9629-1.1968$

H $-5.4610 \quad 4.0882-6.2080$

H $-1.8080 \quad 1.3341 \quad-5.9487$ 